Supporting information

\title{
Pd-Catalyzed Enantioselective Double Heck Reaction
}

\author{
Baihang Ju, Shigui Chen* and Wangqing Kong* \\ E-mail: wqkong@whu.edu.cn
}

The Center for Precision Synthesis, Institute for Advanced Studies, Wuhan University, 299 Bayi Road, Wuhan, Hubei 430072, P. R. China 


\section{Contents}

1. General information S3

2. General procedures $\quad$ S4

3. Optimization of reaction conditions $\quad$ S5

4. General procedure for the synthesis of starting materials S6

5. Characterization data of products S8

6. Copies of the ${ }^{1} \mathrm{H},{ }^{19} \mathrm{~F}$ and ${ }^{13} \mathrm{C}$ NMR spectra S64

$\begin{array}{ll}\text { 7. References } & \mathrm{S} 100\end{array}$ 


\section{General Information}

${ }^{1} \mathrm{H}$ and ${ }^{13} \mathrm{C}$ NMR data were recorded with Bruker ADVANCE III (400 MHz) or JNMECZ400S/L1 (400 MHz) spectrometers. Chemical shifts are given in ppm. The spectra are calibrated to the residual ${ }^{1} \mathrm{H}$ and ${ }^{13} \mathrm{C}$ signals of the solvents. Multiplicities are abbreviated as follows: singlet (s), doublet (d), triplet (t), quartet (q), doublet-doublet (dd), quintet (quint), septet (sept), multiplet (m), and broad (b). ${ }^{19} \mathrm{~F}$ NMR spectra were recorded using $\mathrm{CFCl}_{3}$ as internal standard. Gas chromatography were determined with a SHIMADZU Nexis GC 2030 gas chromatography instrument with a FID detector. High-resolution mass spectra (HRMS) were recorded on DIONEX UltiMate 3000 \& Bruker Compact TOF mass spectrometer. Enantiomeric excesses were determined with a SHIMADZU LC-20ADXR system using chiral stationary phase columns (DAICEL) by comparing the samples with the corresponding racemic samples. Column and elution details were specified in each entry.

Materials and Methods: Unless otherwise stated, starting materials were purchased from commercial suppliers (Adamas-beta ${ }^{\circledR}$, Alfa, Aldrich and so on). All reactions dealing with air- or moisture-sensitive compounds were performed in the argon-filled glove box or by standard Schlenk techniques in oven-dried reaction vessels under argon atmosphere. Solvents were purchased in HPLC quality, degassed by purging thoroughly with argon and dried over activated molecular sieves of appropriate size. More sensitive compounds were stored in a desiccator or in a glove-box if required. Reactions were monitored by thin layer chromatography (TLC) using glass $0.25 \mathrm{~mm}$ silica gel plates. Compounds were visualized by UV-light at $254 \mathrm{~nm}$ and by dipping the plates in an aqueous potassium permanganate solution followed by heating. Flash column chromatography was performed over silica gel (200-400 mesh). 


\section{General Procedure}

General procedure for the enantioselective aryl-alkenylation of arenediazonium salts 1 with alkenes 2:
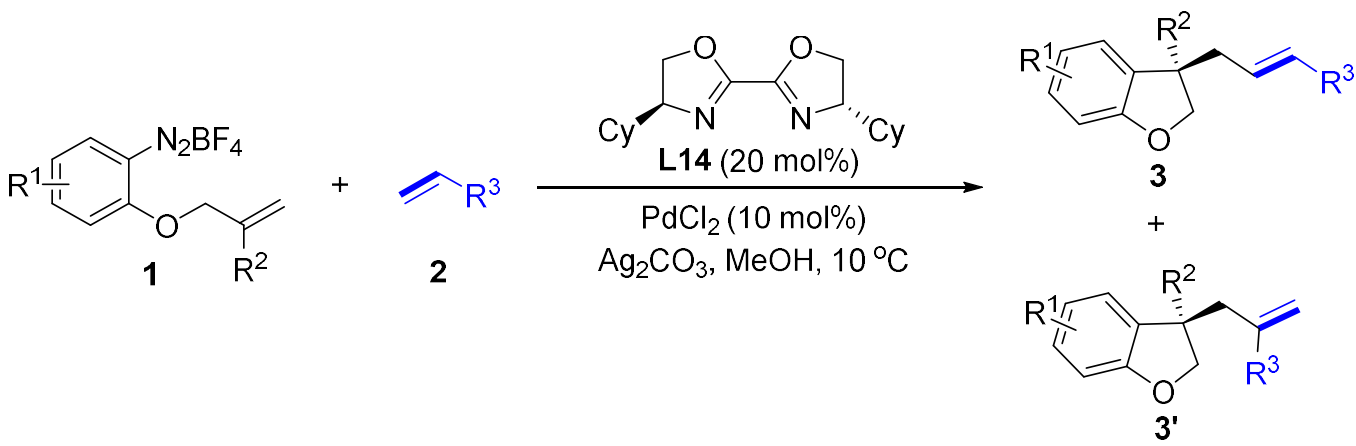

A mixture of $\mathrm{PdCl}_{2}(10 \mathrm{~mol} \%, 1.7 \mathrm{mg}),(4 \mathrm{~S}, 4$ 'S)-BOX L14 $(20 \mathrm{~mol} \%, 6.0 \mathrm{mg})$ and $\mathrm{MeOH}(1.0 \mathrm{~mL})$ was stirred in an oven-dried vial at room temperature for 15 minutes to form the precatalyst. The resulting solution was cooled to $10^{\circ} \mathrm{C}$ and charged with arenediazonium salt 1 (0.1 mmol, 1.0 equiv), alkene $2(0.2 \mathrm{mmol}, 0.2$ equiv) and $\mathrm{Ag}_{2} \mathrm{CO}_{3}(0.1 \mathrm{mmol}, 27.5 \mathrm{mg})$. Then the reaction mixture was stirred at the same temperature until the reaction was completed. The solvent was removed under vacuum and the residue was then purified by column chromatography on silica gel, eluting with petroleum ether/EtOAc $=100: 1 \sim 5 / 1$ to afford the desired dihydrobenzofuran 3 (This reaction show high regioselectivity and in almost all cases only $<5 \%$ of regioisomers 3' were detected). 


\section{Optimization of Reaction Conditions}

\section{Ligand screening:}

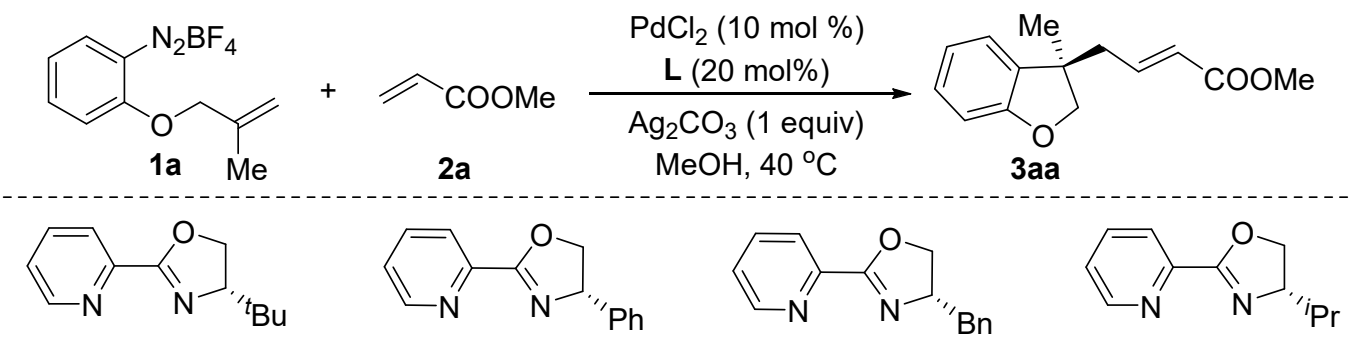

$90 \%$ y, $74 \%$ ee

trace $14 \%$ y, $67 \%$ ee

$71 \%$ y, 69\% ee

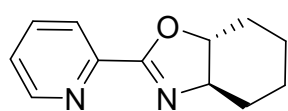

$22 \%$ y, $4 \%$ ee<smiles>CC(C)[C@@H]1COC(c2ccc(C(F)(F)F)cn2)=N1</smiles>

$84 \%$ y, $76 \%$ ee<smiles>FC(F)(F)c1ccc(C2=NC3c4ccccc4C[C@H]3O2)nc1</smiles>

$84 \%$ y, $59 \%$ ee<smiles>COc1cc(C2=NC([13C](C)C)CO2)nc2ccccc12</smiles>

$36 \%$ y, $16 \%$ ee<smiles>CCC[C@@H]1COC(C2=N[C@@H](P)CO2)=N1</smiles>

$52 \%$ y, $81 \%$ ee<smiles>Br[C@H]1COC(C2=N[C@H](Br)CO2)=N1</smiles>

$87 \%$ y, $69 \%$ ee<smiles>[Al]C1COC(c2ccccn2)=N1</smiles>

$56 \%$ y, $70 \%$ ee<smiles>c1ccc(C2=N[C@H]3c4ccccc4C[C@H]3O2)nc1</smiles>

$31 \%$ y, $73 \%$ ee

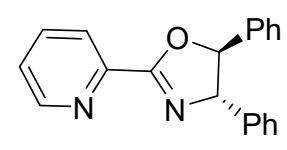

$16 \%$ y, $5 \%$ ee<smiles>FC(F)(F)c1ccc(C2=N[C@H](Cc3ccccc3)CO2)nc1</smiles>

$82 \%$ y, $76 \%$ ee<smiles>CC(C)(C)[C@H]1COC(c2ccc3ccccc3n2)=N1</smiles>

$83 \%$ y, $67 \%$ ee<smiles>CC(C)(C1=NC(c2ccccc2)CO1)C1=NC(c2ccccc2)CO1</smiles>

$89 \%$ y, $1 \%$ ee<smiles>[Al]C1COC(C2=N[C@@H]([AlH2])CO2)=N1</smiles>

$65 \%$ y, $82 \%$ ee<smiles>c1ccc([C@H]2COC(C3=N[C@@H](c4ccccc4)CO3)=N2)cc1</smiles>

$85 \%$ y, $48 \%$ ee

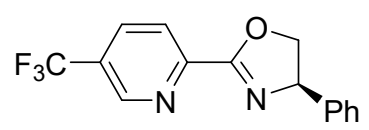

$70 \%$ y, 50\% ee
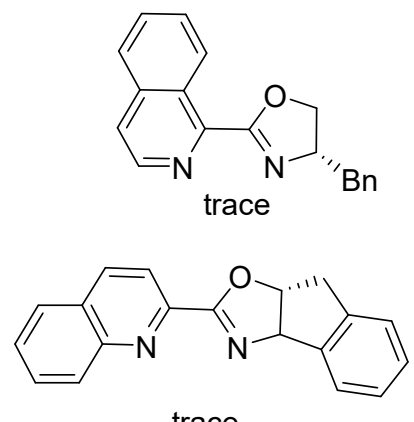

trace<smiles>CC(C)[13CH2][C@@H]1COC(C2=N[C@@H]([13CH3])CO2)=N1</smiles>

$62 \%$ y, $17 \%$ ee<smiles>CC(C)C[C@@H]1COC(c2cnc(C3=NC([11C](C)C)CO3)cn2)=N1</smiles>

$57 \%$ y, $79 \%$ ee 


\section{General procedure for the synthesis of starting materials}

\subsection{General procedure for allylation of 2-nitrophenols}

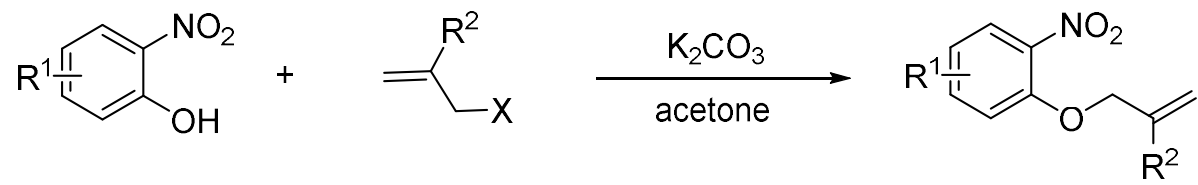

To an oven-dried sealed tube containing 2-nitrophenol (20 mmol, 1.0 equiv), $\mathrm{K}_{2} \mathrm{CO}_{3}$ (3.0 equiv, $8.29 \mathrm{~g})$ in acetone $(60 \mathrm{~mL})$ at $0{ }^{\circ} \mathrm{C}$ was added the corresponding allyl halide $(\mathrm{X}=\mathrm{Br}, 1.2$ equiv; $\mathrm{X}=\mathrm{Cl}, 1.5$ equiv). The tube was sealed and the reaction mixture was heated at $65^{\circ} \mathrm{C}$ until the reaction was completed. The mixture was cooled to room temperature, quenched by the addition of $\mathrm{H}_{2} \mathrm{O}$ and extracted with EtOAc. The combined organic layers was washed with saturated $\mathrm{NH}_{4} \mathrm{Cl}$ and dried over $\mathrm{Na}_{2} \mathrm{SO}_{4}$. The solvent was then removed under vacuum to give the desired alkylation product in an almost pure form, which was used in the next step without further purification.

\subsection{General procedure for reduction of nitro to aniline}<smiles>[R]C(=C)COc1cc[R1]cc1N</smiles>

To a vigorously stirred solution of the above prepared allylated nitro compound (10 mmol, 1.0 equiv) in $\mathrm{MeOH}(60 \mathrm{~mL})$ and saturated $\mathrm{NH}_{4} \mathrm{Cl}$ aqueous solution $(5 \mathrm{~mL})$ was added $\mathrm{Zn}$ dust (15 equiv, $9.75 \mathrm{~g}$ ). After the reaction was completed, the reaction mixture was filtered through a short pad of celite and washed with EtOAc. The filtrate was dried over $\mathrm{Na}_{2} \mathrm{SO}_{4}$ and the solvent was then removed under vacuum to give the corresponding aniline derivative, which was used in the next step without further purification. 


\subsection{General procedure for the synthesis of arenediazonium tetrafluoroborates $1^{1}$}
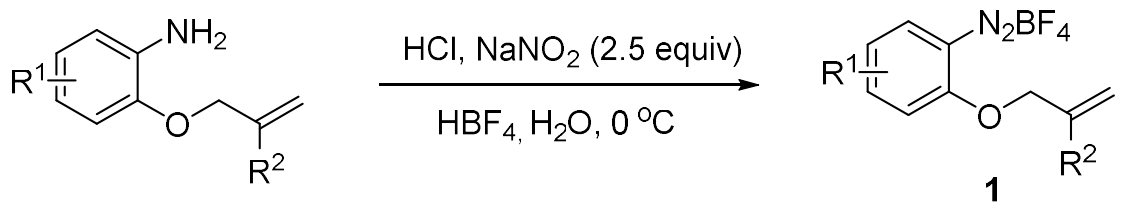

A mixture of the above aniline (10 mmol, 1 equiv) and $\mathrm{HCl}$ (3 equiv, $3.0 \mathrm{~mol} / \mathrm{L}, 10 \mathrm{~mL}$ ) was stirred at $0{ }^{\circ} \mathrm{C}$ for 10 minutes. To the vigorously stirred suspension was added a solution of $\mathrm{NaNO}_{2}$ (2.5 equiv, $1.73 \mathrm{~g}$ ) in $2.0 \mathrm{~mL}$ of $\mathrm{H}_{2} \mathrm{O}$ at the same temperature for 20 minutes. The reaction mixture was washed with cold $\mathrm{Et}_{2} \mathrm{O}$, followed by the addition of $\mathrm{HBF}_{4}(48 \% \mathrm{w} / \mathrm{w}, 4.5$ equiv, $5.9 \mathrm{~mL})$. This solution was extracted with $\mathrm{DCM}$, the organic phase was washed with $\mathrm{H}_{2} \mathrm{O}$ and dried over anhydrous $\mathrm{Na}_{2} \mathrm{SO}_{4}$. The solvent was evaporated under vacuum to furnish the corresponding arenediazonium salt. Depending on the purity of the salt, it was recrystallized by dissolution in acetone followed by the addition of cold $\mathrm{Et}_{2} \mathrm{O}$ at $0{ }^{\circ} \mathrm{C}$. All the arenediazonium tetrafluoroborate salts were stored in a freezer at $-25^{\circ} \mathrm{C}$. 


\section{Characterization data of products}

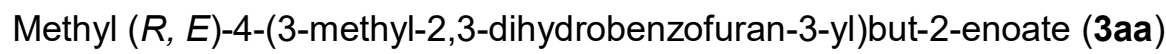

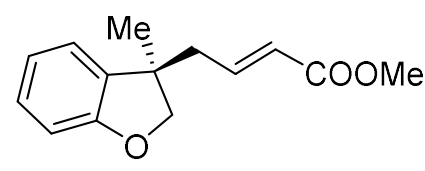

Chemical Formula: $\mathrm{C}_{14} \mathrm{H}_{16} \mathrm{O}_{3}$

Exact Mass: 232.1099

3aa was prepared according to general procedure using 1 a $(0.1 \mathrm{mmol}, 1.0$ equiv, 26.2 $\mathrm{mg})$ and $2 \mathrm{a}(0.2 \mathrm{mmol}, 18 \mu \mathrm{L})$ and was purified by silica gel column chromatography (petroleum ether/EtOAc $=50 / 1 \sim 10 / 1)$ to obtain 3aa as colorless oil $(18.4 \mathrm{mg}, 79 \%$ yield). The ${ }^{1} \mathrm{H}$ NMR data matched those reported in the literature ${ }^{2}:{ }^{1} \mathrm{H}$ NMR $(400 \mathrm{MHz}$, $\left.\mathrm{CDCl}_{3}\right):$ $\delta$ 7.17-7.08 (m, 2H), 6.92-6.78 (m, 3H), $5.85(\mathrm{dt}, J=15.5,1.4 \mathrm{~Hz}, 1 \mathrm{H}), 4.35(\mathrm{~d}$, $J=8.8 \mathrm{~Hz}, 1 \mathrm{H}), 4.15(\mathrm{~d}, J=8.8 \mathrm{~Hz}, 1 \mathrm{H}), 3.72(\mathrm{~s}, 3 \mathrm{H}), 2.50(\mathrm{dd}, J=7.8,1.4 \mathrm{~Hz}, 2 \mathrm{H})$, $1.38(\mathrm{~s}, 3 \mathrm{H})$;

${ }^{13} \mathrm{C}$ NMR $\left(101 \mathrm{MHz}, \mathrm{CDCl}_{3}\right): \delta 166.5,159.2,144.3,133.9,128.5,124.2,122.8,120.7$, $109.9,81.7,51.5,45.2,43.3,24.8$.

The enantiomeric purity was established by HPLC analysis using a chiral column: OJ$\mathrm{H}$ column, $30^{\circ} \mathrm{C}, n$-Hexane/i-Propanol $=97 / 3$ as eluent, $254 \mathrm{~nm}, 1 \mathrm{~mL} / \mathrm{min} . \mathrm{tR}=13$ $\min$ (minor), $14 \mathrm{~min}$ (major).

Optical Rotation: $[\alpha]_{D^{30}} 25.2$ (c $0.1, \mathrm{CHCl}_{3}$ ) for $94 \%$ ee.

Absolute stereochemistry was determined through analogy with 3ac. 

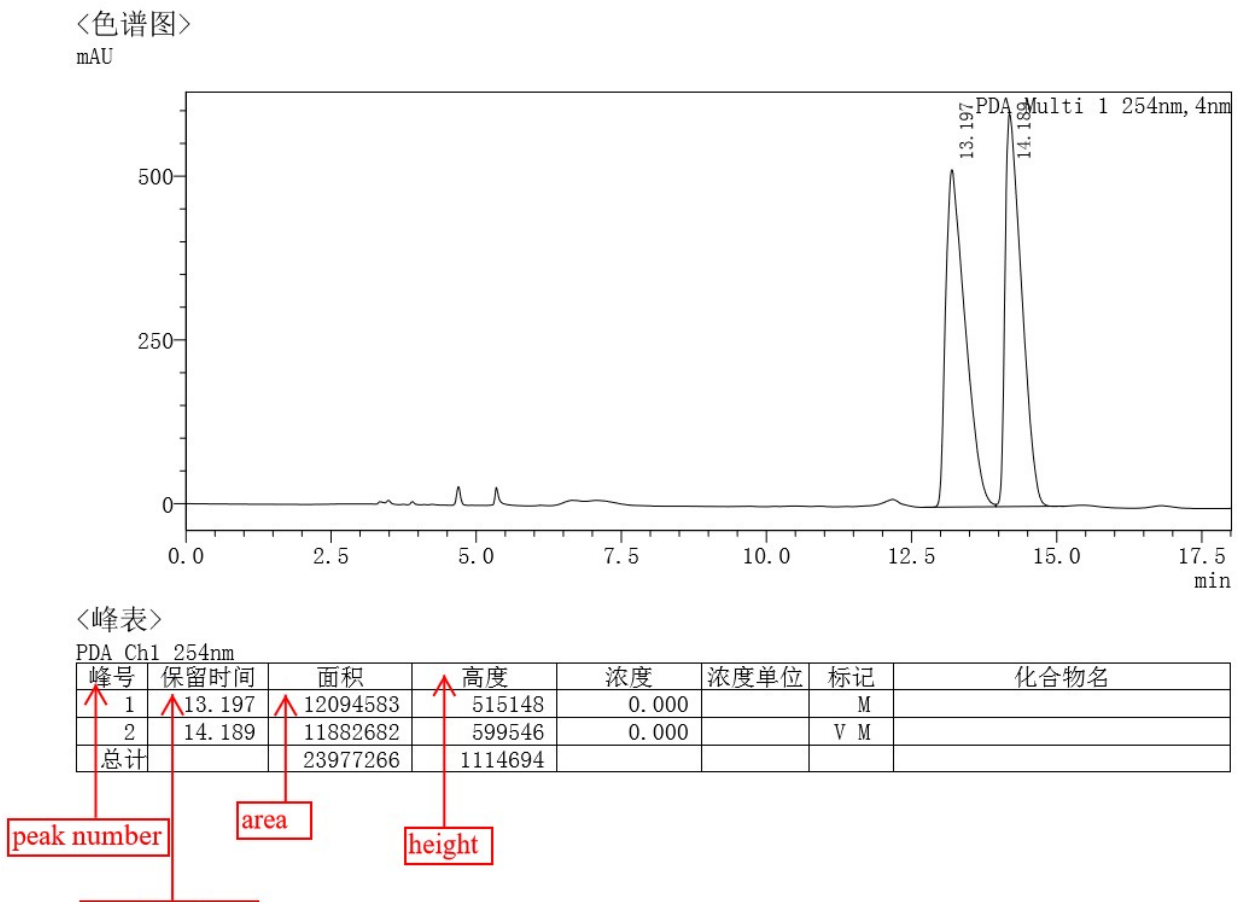

retention time

〈色谱图〉

$\mathrm{mAU}$

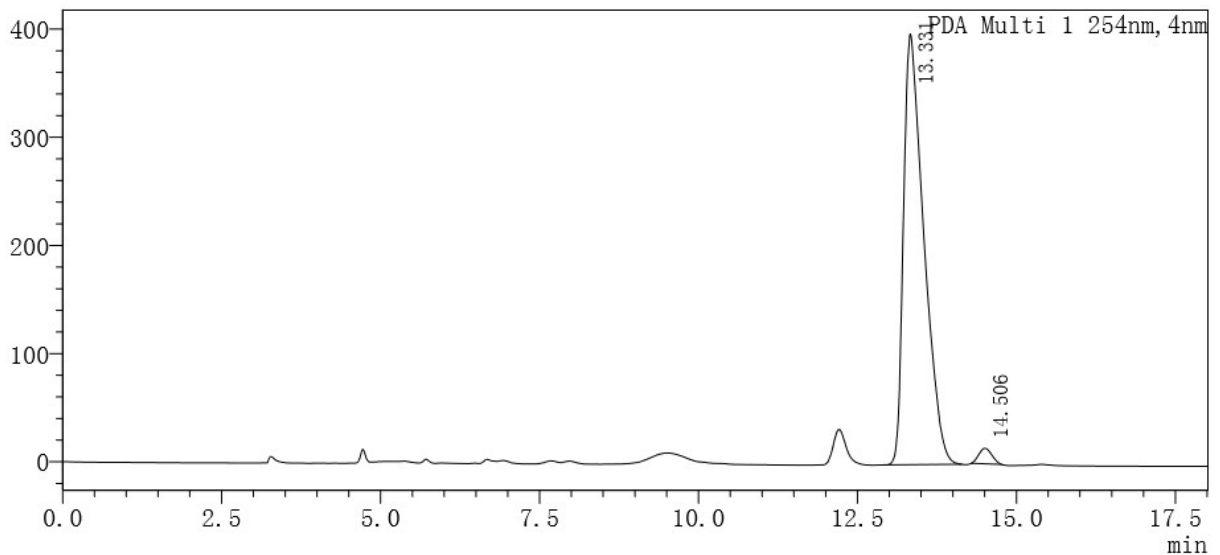

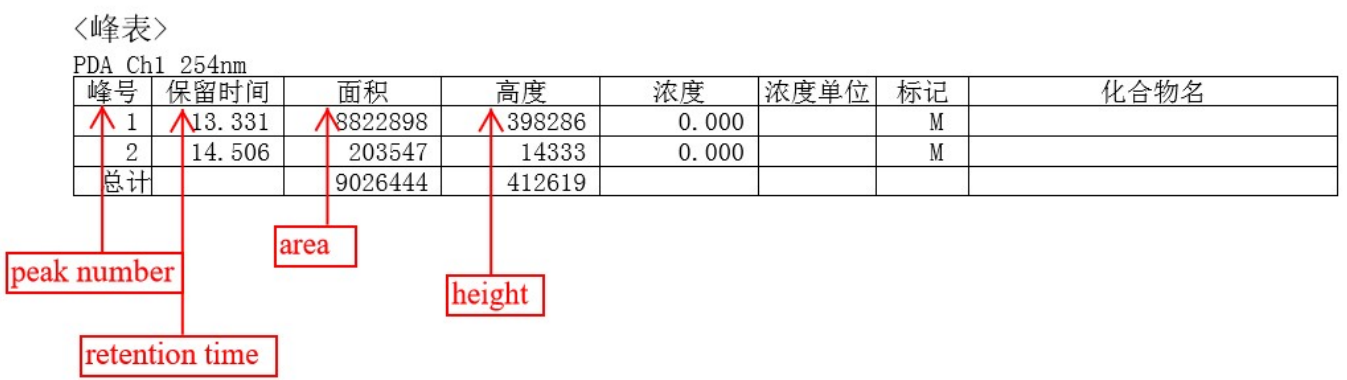


tert-butyl ( $R, E)$-4-(3-methyl-2,3-dihydrobenzofuran-3-yl)but-2-enoate (3ab)

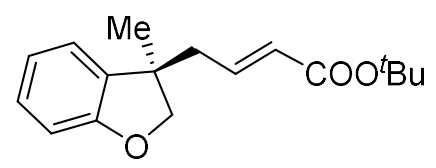

Chemical Formula: $\mathrm{C}_{17} \mathrm{H}_{22} \mathrm{O}_{3}$

Exact Mass: 274.1569

3ab was prepared according to general procedure using 1 a $(0.1 \mathrm{mmol}, 1.0$ equiv, 26.2 $\mathrm{mg})$ and $\mathbf{2 b}(0.2 \mathrm{mmol}, 29 \mu \mathrm{L})$ and was purified by silica gel column chromatography (petroleum ether/EtOAc $=50 / 1 \sim 10 / 1)$ to obtain $3 \mathrm{ab}$ as colorless oil $(20.6 \mathrm{mg}, 75 \%$ yield). ${ }^{1} \mathrm{H}$ NMR $\left(400 \mathrm{MHz}, \mathrm{CDCl}_{3}\right): \delta 7.16-7.08(\mathrm{~m}, 2 \mathrm{H}), 6.91-6.87$ (td, $J=7.4,0.8 \mathrm{~Hz}$, 1H), 6.81-6.72 (m, 2H), $5.79(\mathrm{~d}, J=15.5 \mathrm{~Hz}, 1 \mathrm{H}), 4.35(\mathrm{~d}, J=8.8 \mathrm{~Hz}, 1 \mathrm{H}), 4.14(\mathrm{~d}, J=$ $8.8 \mathrm{~Hz}, 1 \mathrm{H}), 2.47(\mathrm{~d}, J=7.7 \mathrm{~Hz}, 2 \mathrm{H}), 1.47(\mathrm{~s}, 9 \mathrm{H}), 1.37$ (s, 3H);

${ }^{13} \mathrm{C} \mathrm{NMR}\left(101 \mathrm{MHz}, \mathrm{CDCl}_{3}\right): \delta$ 165.5, 159.3, 142.8, 134.2, 128.4, 126.3, 122.8, 120.6, $109.9,81.8,80.3,45.2,43.1,28.1,24.7$

HRMS: (ESI) calcd for $\mathrm{C}_{17} \mathrm{H}_{22} \mathrm{NaO}_{3}{ }^{+}[\mathrm{M}+\mathrm{Na}]^{+} 297.1461$; found 297.1456.

The enantiomeric purity was established by HPLC analysis using a chiral column: OJ$\mathrm{H}$ column, $30{ }^{\circ} \mathrm{C}, n$-Hexane $/ i$-Propanol $=97 / 3$ as eluent, $254 \mathrm{~nm}, 1 \mathrm{~mL} / \mathrm{min}$. $\mathrm{tR}=5.3$ $\min$ (major), $6.9 \mathrm{~min}$ (minor).

Optical Rotation: $[\alpha]_{D}{ }^{28} 8.0$ (c $\left.0.3, \mathrm{CHCl}_{3}\right)$ for $99 \%$ ee. Absolute stereochemistry was determined through analogy with 3ac. 

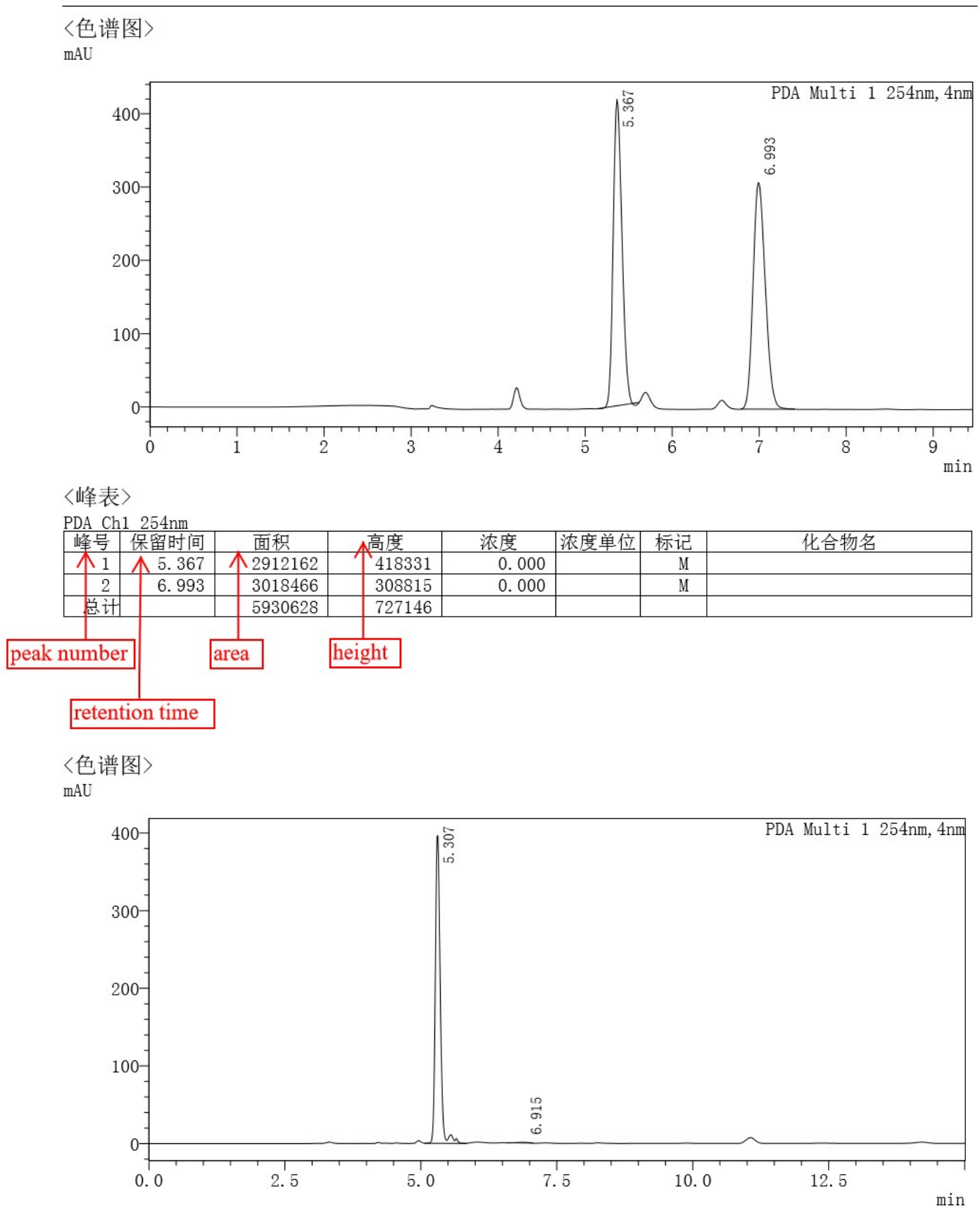

〈峰表〉

PDA Ch1 254nm

\begin{tabular}{|c|c|c|c|c|c|c|c|}
\hline 峰号 & 保留时间 & 面积 & 高度 & 浓度 & 浓度单位 & 标记 & 化合物名 \\
\hline 木 1 & \$5. 307 & 木2494425 & 396323 & 0.000 & & $\mathrm{M}$ & \\
\hline 2 & 6.915 & 12717 & 922 & 0.000 & & $\mathrm{M}$ & \\
\hline 总计 & & 2507142 & 397245 & & & & \\
\hline
\end{tabular}

peak number

area height

retention time 
(R)-3-cinnamyl-3-methyl-2,3-dihydrobenzofuran (3ac)<smiles>C[C@]1(C/C=C/c2ccccc2)COc2ccccc21</smiles>

Chemical Formula: $\mathrm{C}_{18} \mathrm{H}_{18} \mathrm{O}$

Exact Mass: 250.1358

3ac was prepared according to general procedure using $1 \mathrm{a}(0.1 \mathrm{mmol}, 1.0$ equiv, 26.2 $\mathrm{mg})$ and $2 \mathrm{c}(0.2 \mathrm{mmol}, 23 \mu \mathrm{L})$ and was purified by silica gel column chromatography (petroleum ether/EtOAc $=100 / 1 \sim 30 / 1)$ to obtain 3ac as colorless oil $(19.0 \mathrm{mg}, 76 \%$ yield). The ${ }^{1} \mathrm{H}$ NMR data matched those reported in the literature ${ }^{3}:{ }^{1} \mathrm{H}$ NMR $(400 \mathrm{MHz}$, $\left.\mathrm{CDCl}_{3}\right): \delta$ 7.34-7.28 (m, 4H), 7.25-7.20 (m, 1H), 7.19-7.12 (m, 2H), 6.91 (td, J = 7.4, $0.9 \mathrm{~Hz}, 1 \mathrm{H}), 6.82(\mathrm{dt}, J=7.9,0.8 \mathrm{~Hz}, 1 \mathrm{H}), 6.43(\mathrm{dt}, J=15.7,1.3 \mathrm{~Hz}, 1 \mathrm{H}), 6.12(\mathrm{dt}, J=$ 15.8, 7.5 Hz, 1H), 4.46 (d, J = 8.6 Hz, 1H), $4.17(\mathrm{~d}, J=8.6 \mathrm{~Hz}, 1 \mathrm{H}), 2.52$ (dd, $J=7.5$, $1.4 \mathrm{~Hz}, 2 \mathrm{H}), 1.41(\mathrm{~s}, 3 \mathrm{H})$;

${ }^{13} \mathrm{C}$ NMR (101 MHz, $\left.\mathrm{CDCl}_{3}\right): \delta$ 159.5, 137.3, 134.8, 133.4, 128.5, 128.2, 127.2, 126.1, $125.6,122.9,120.5,109.7,82.0,45.6,44.2,25.0$.

The enantiomeric purity was established by HPLC analysis using a chiral column: OD$\mathrm{H}$ column, $30{ }^{\circ} \mathrm{C}, n$-Hexane/i-Propanol $=98 / 2$ as eluent, $254 \mathrm{~nm}, 1 \mathrm{~mL} / \mathrm{min} . \mathrm{tR}=5.6$ $\min$ (minor), $5.8 \mathrm{~min}$ (major).

Optical Rotation: $[\alpha]_{D}{ }^{30} 21.3$ (c $0.1, \mathrm{CHCl}_{3}$ ) for $94 \%$ ee.

The absolute configuration of product 3ac was determined by converting 3ac into the corresponding known compound $\mathbf{6}^{4}$ by ozonolysis of alkene to aldehyde, followed by olefination of the resulting aldehyde to terminal alkene and hydrogenation. All other dihydrobenzofurans $\mathbf{3}$ were attributed accordingly.

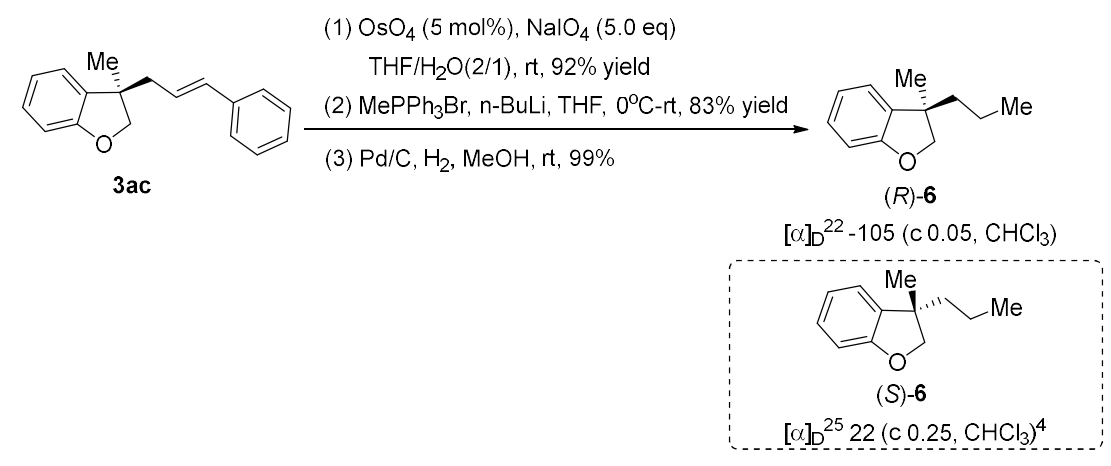


〈色谱图〉

$\mathrm{mAU}$

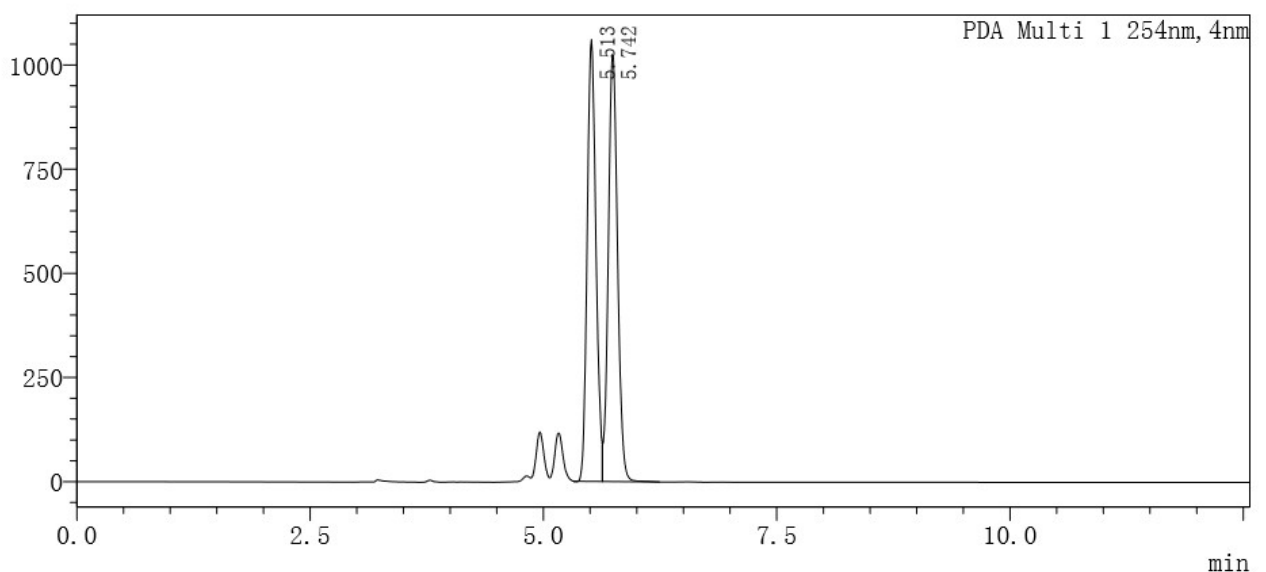

\begin{tabular}{|c|c|c|c|c|c|c|c|}
\hline \multicolumn{8}{|c|}{ 〈峰表〉 } \\
\hline 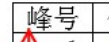 & 保留时间 & & 我度 & 浓度 & 浓度单位 & 标记 & 化合物名 \\
\hline $\begin{array}{ll} & 1 \\
\end{array}$ & 木5.513 & 16925224 & 1060933 & 0.000 & & M & \\
\hline 2 & 5.742 & 7072695 & 1025268 & 0.000 & & V M & \\
\hline 总计 & & 13997920 & 2086201 & & & & \\
\hline$\frac{1}{\text { number }}$ & are & & eight & & & & \\
\hline
\end{tabular}

retention time

〈色谱图〉

$\mathrm{mAU}$

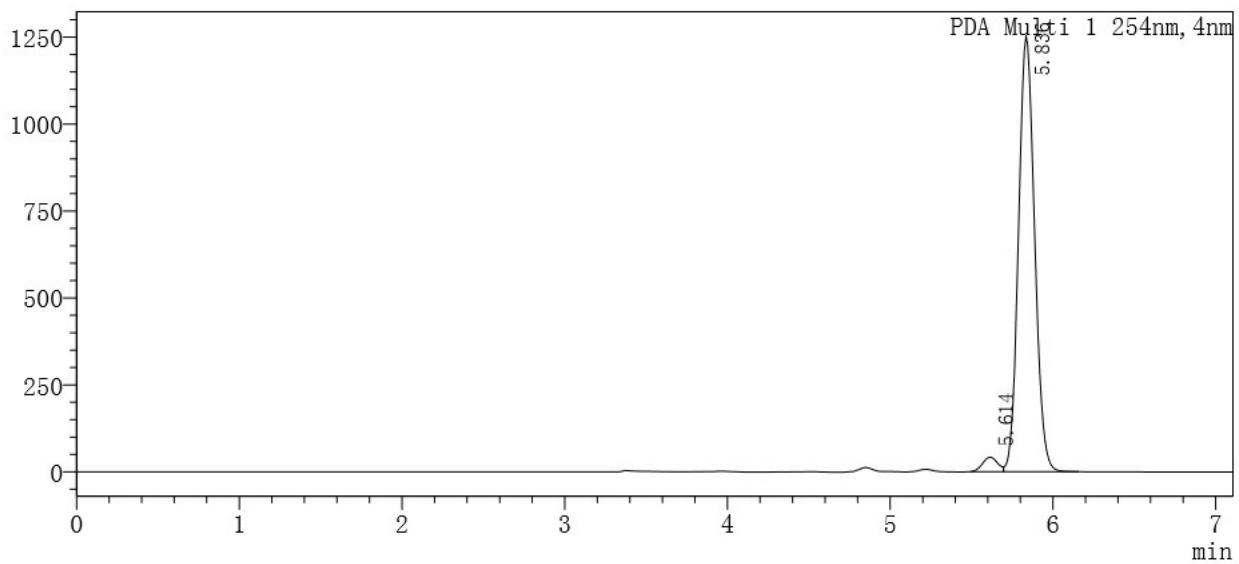

〈峰表〉

PDA Ch1 254nm

峰号

\begin{tabular}{|l|l|l|} 
本 1 & \5.614 & \ 272153 \\
\hline
\end{tabular}

\begin{tabular}{|l|l|r|}
2 & 5.836 & 8610817 \\
\hline 总计 & & 8882970
\end{tabular}

\begin{tabular}{|c|c|c|c|c|c|c|c|}
\hline & 2 & 5.836 & 8610817 & 1253693 & 0.000 & V M & \\
\cline { 1 - 6 } & & 8882970 & & 1295452 & & & \\
\hline
\end{tabular}

1295452

浓度
0.000
0.000

浓度单位 标记 1 化合物名

retention time 
(R, E)-3-methyl-3-(3-(p-tolyl)allyl)-2,3-dihydrobenzofuran (3ad)

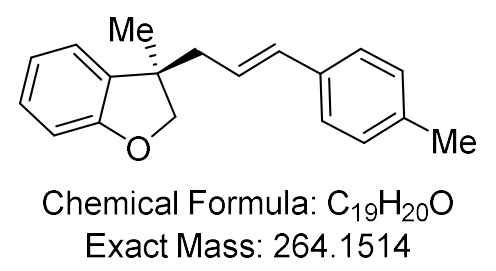

3ad was prepared according to general procedure using $1 \mathrm{a}(0.1 \mathrm{mmol}, 1.0$ equiv, 26.2 $\mathrm{mg}$ ) and $\mathbf{2 d}(0.2 \mathrm{mmol}, 26 \mu \mathrm{L})$ and was purified by silica gel column chromatography (petroleum ether/EtOAc $=100 / 1 \sim 30 / 1)$ to obtain 3ad as colorless oil $(18.1 \mathrm{mg}, 66 \%$ yield). ${ }^{1} \mathrm{H}$ NMR $\left(400 \mathrm{MHz}, \mathrm{CDCl}_{3}\right): \delta$ 7.33-7.07 (m, 6H), 6.98-6.90 (m, 1H), $6.84(\mathrm{~d}, J=$ $7.9 \mathrm{~Hz}, 1 \mathrm{H}), 6.42(\mathrm{~d}, J=15.7 \mathrm{~Hz}, 1 \mathrm{H}), 6.18-5.99(\mathrm{~m}, 1 \mathrm{H}), 4.48(\mathrm{dd}, J=8.6, \mathrm{~Hz}, 1 \mathrm{H})$, 4.19 (dd, J = 8.6, Hz, 1H), $2.53(\mathrm{~d}, J=7.3 \mathrm{~Hz}, 2 \mathrm{H}), 2.37$ (s, 3H), 1.43 (s, 3H);

${ }^{13} \mathrm{C}$ NMR $\left(101 \mathrm{MHz}, \mathrm{CDCl}_{3}\right): \delta$ 159.4, 137.0, 134.9, 134.5, 133.2, 129.2, 128.1, 125.9, $124.5,122.9,120.4,109.7,81.9,45.6,44.2,24.9,21.2$;

HRMS: (ESI) calcd for $\mathrm{C}_{19} \mathrm{H}_{20} \mathrm{NaO}^{+}[\mathrm{M}+\mathrm{Na}]^{+} 287.1406$; found 287.1407 .

The enantiomeric purity was established by HPLC analysis using a chiral column: OD$\mathrm{H}$ column, $30{ }^{\circ} \mathrm{C}, n$-Hexane $/ i$-Propanol $=97 / 3$ as eluent, $254 \mathrm{~nm}, 1 \mathrm{~mL} / \mathrm{min}$. $\mathrm{tR}=4.5$ $\min$ (minor), $5.0 \mathrm{~min}$ (major).

Optical Rotation: $[\alpha]_{\mathrm{D}}{ }^{30}-9.3$ (c $\left.0.2, \mathrm{CHCl}_{3}\right)$ for $94 \%$ ee. Absolute stereochemistry was determined through analogy with 3ac. 


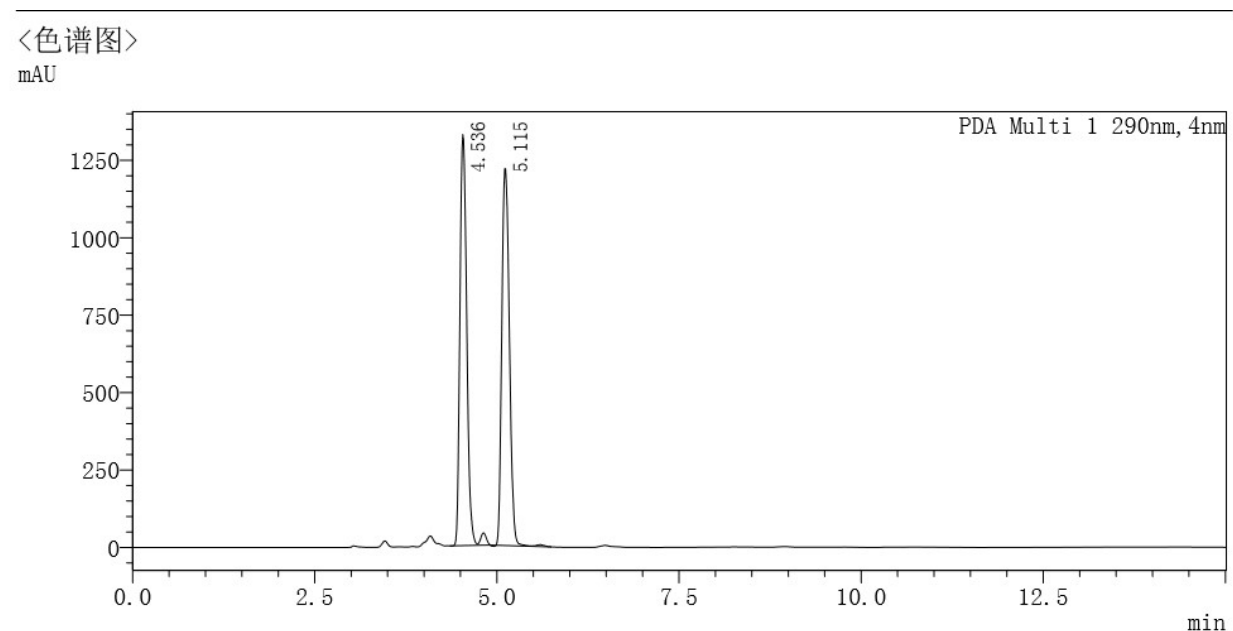

〈峰表〉

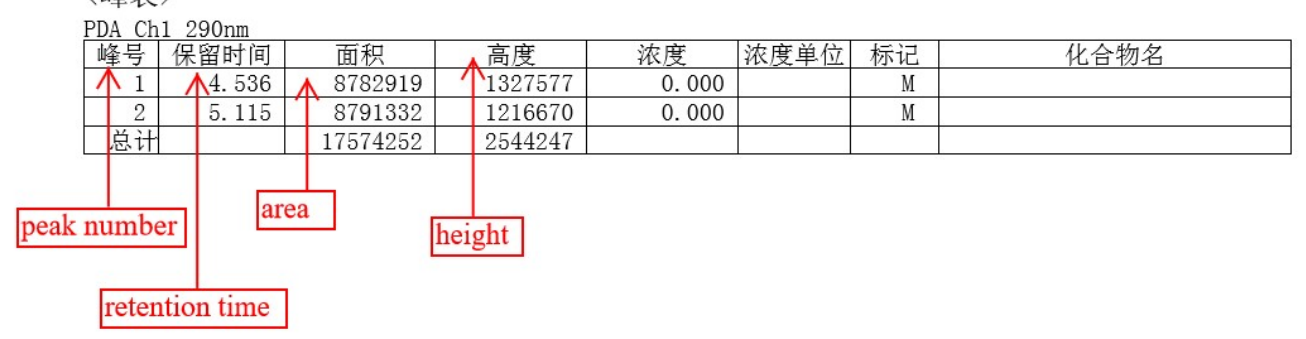

〈色谱图〉

$\mathrm{mAU}$

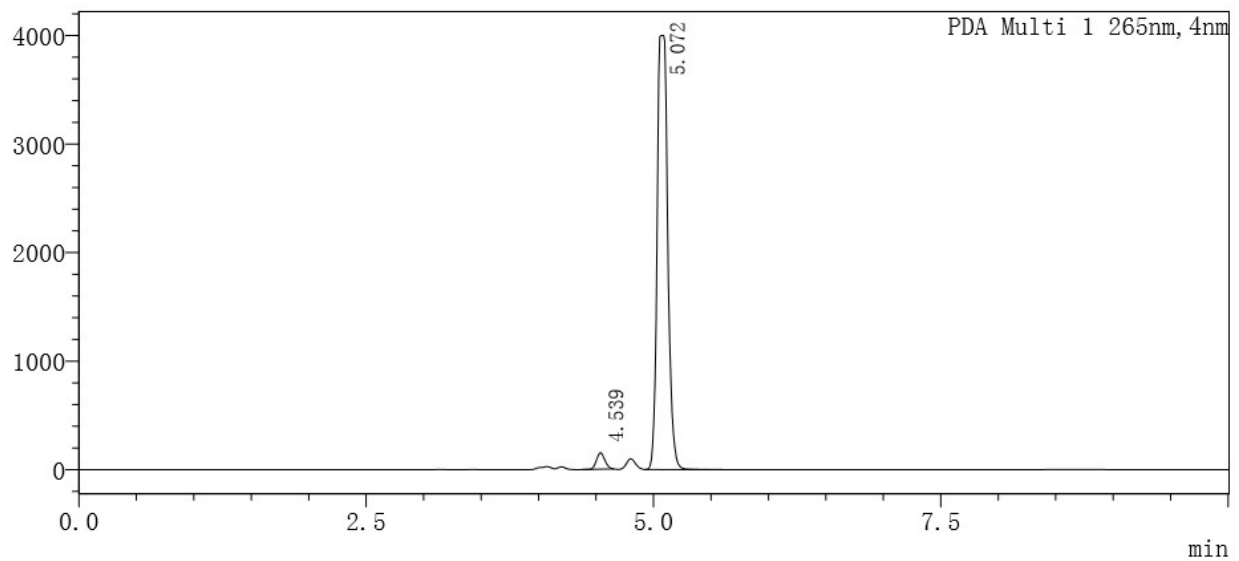

〈峰表〉

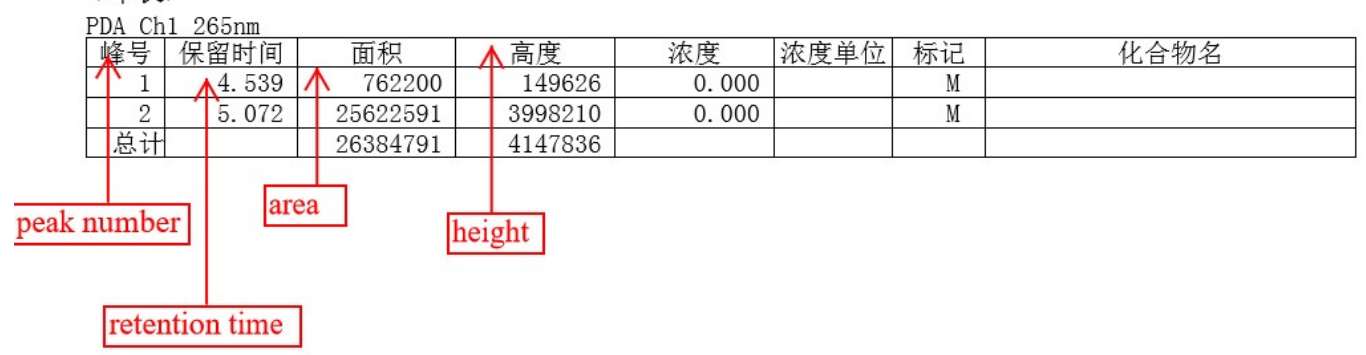


(R, E)-3-(3-(4-methoxyphenyl)allyl)-3-methyl-2,3-dihydrobenzofuran (3ae)

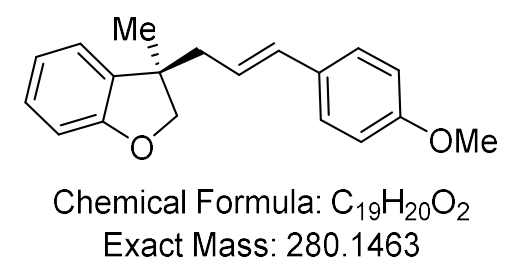

3ae was prepared according to general procedure using $1 \mathrm{a}(0.1 \mathrm{mmol}, 1.0$ equiv, 26.2 $\mathrm{mg})$ and $2 \mathbf{e}(0.2 \mathrm{mmol}, 26.6 \mu \mathrm{L})$ and was purified by silica gel column chromatography (petroleum ether/EtOAc $=100 / 1 \sim 20 / 1)$ to obtain 3ae as colorless oil $(19.1 \mathrm{mg}, 68 \%$ yield). ${ }^{1} \mathrm{H}$ NMR (400 MHz, $\left.\mathrm{CDCl}_{3}\right): \delta$ 7.25-7.23 (m, 1H), 7.17-7.11 (m, 2H), 6.91-6.80 (m, 5H), $6.36(\mathrm{~d}, J=15.7 \mathrm{~Hz}, 1 \mathrm{H}), 6.01-5.93(\mathrm{~m}, 1 \mathrm{H}), 4.44(\mathrm{~d}, J=8.6 \mathrm{~Hz}, 1 \mathrm{H}), 4.15(\mathrm{~d}$, $J=8.6 \mathrm{~Hz}, 1 \mathrm{H}), 3.80(\mathrm{~s}, 3 \mathrm{H}), 2.48(\mathrm{dd}, J=7.5,1.2 \mathrm{~Hz}, 2 \mathrm{H}), 1.39(\mathrm{~s}, 3 \mathrm{H})$;

${ }^{13} \mathrm{C} \mathrm{NMR}\left(101 \mathrm{MHz}, \mathrm{CDCl}_{3}\right) \delta 159.5,158.9,134.9,132.8,130.2,128.1,127.2,123.4$, $122.9,120.4,113.9,109.7,82.0,55.3,45.7,44.2,25.0$;

HRMS: (ESI) calcd for $\mathrm{C}_{19} \mathrm{H}_{21} \mathrm{O}_{2}{ }^{+}[\mathrm{M}+\mathrm{H}]^{+} 281.1536$; found 281.1540 .

The enantiomeric purity was established by HPLC analysis using a chiral column: OD$\mathrm{H}$ column, $30{ }^{\circ} \mathrm{C}, n$-Hexane/i-Propanol $=99 / 1$ as eluent, $254 \mathrm{~nm}, 0.5 \mathrm{~mL} / \mathrm{min} . \mathrm{tR}=14$ $\min$ (minor), $16 \min$ (major).

Optical Rotation: $[\alpha]_{\mathrm{D}}{ }^{28} 18.4$ (c $\left.0.2, \mathrm{CHCl}_{3}\right)$ for $94 \%$ ee. Absolute stereochemistry was determined through analogy with 3ac. 
〈色谱图〉

$\mathrm{mAU}$

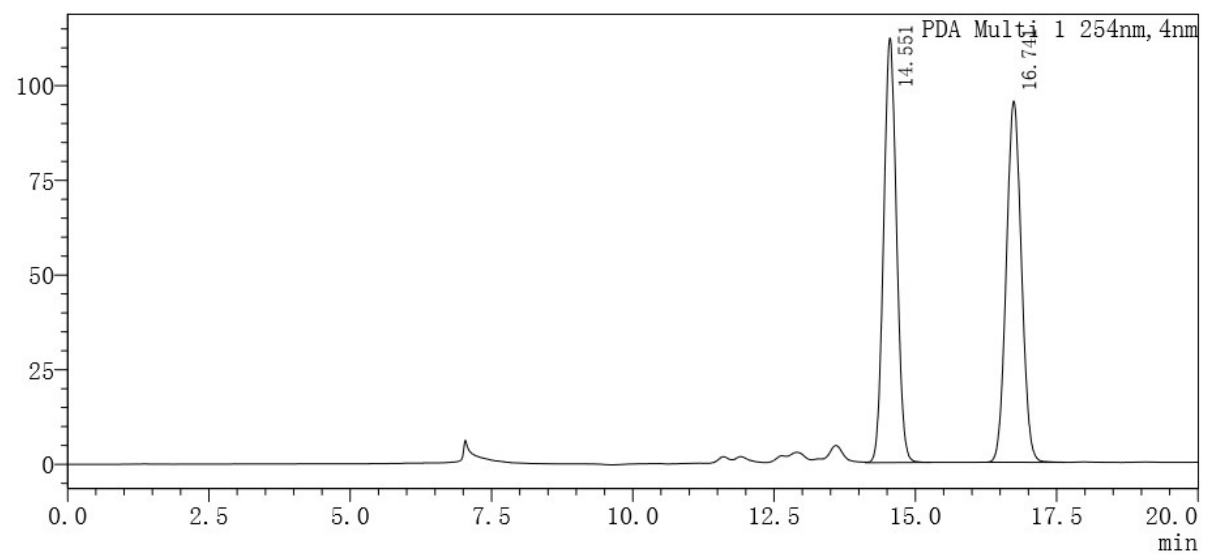

\section{〈峰表〉}

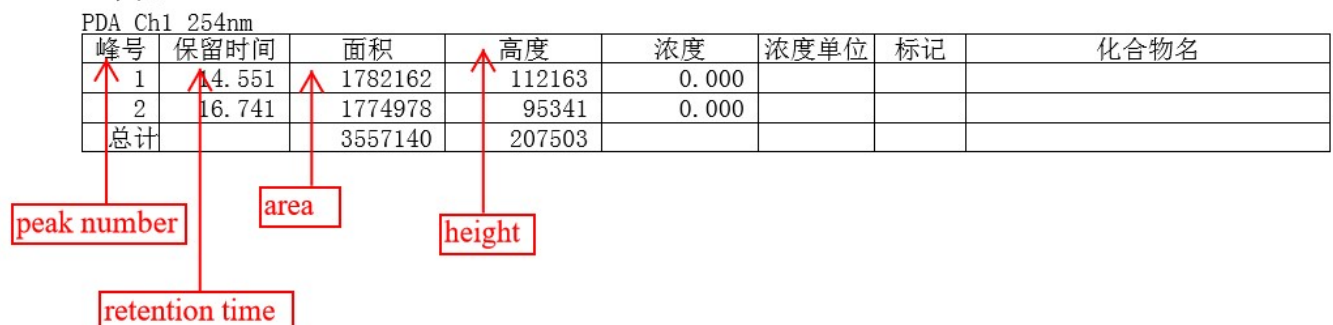

retention time
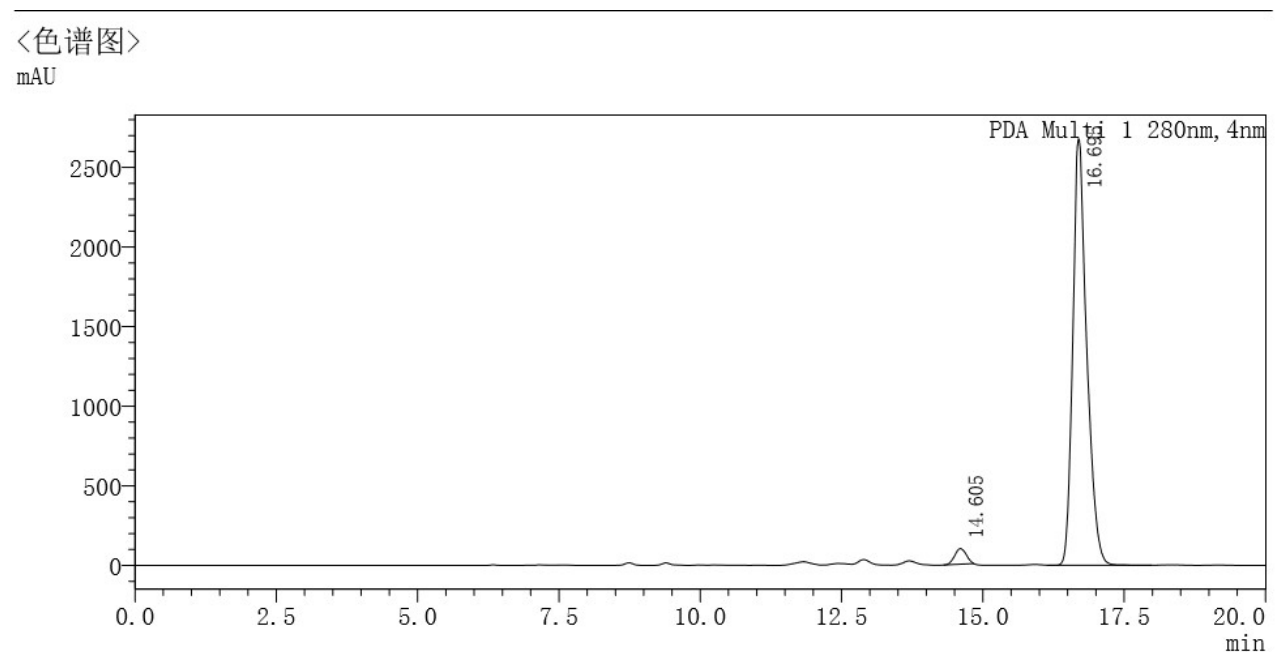

〈峰表〉

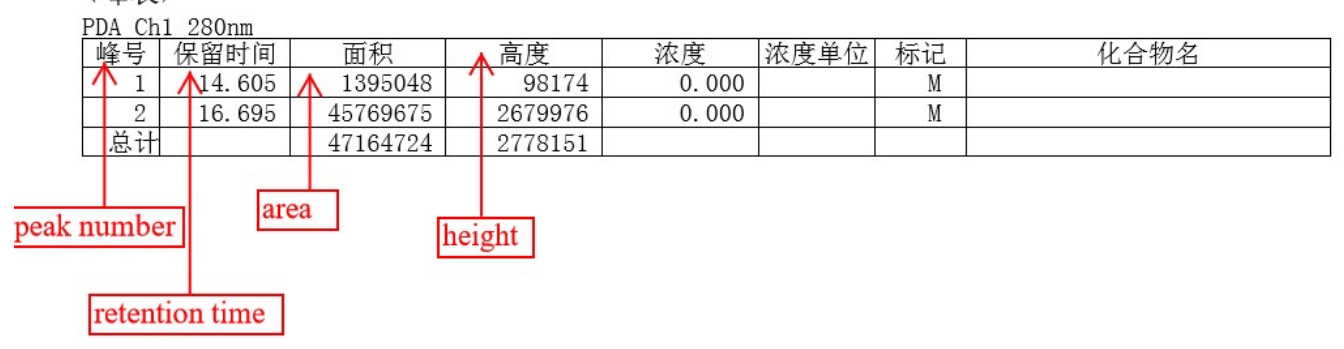




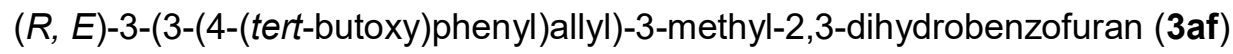

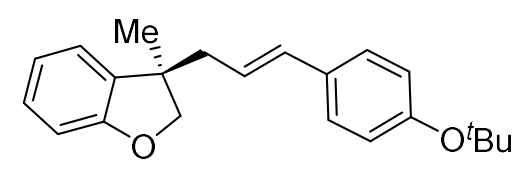

Chemical Formula: $\mathrm{C}_{22} \mathrm{H}_{26} \mathrm{O}_{2}$

Exact Mass: 322.1933

3af was prepared according to general procedure using 1 a $(0.1 \mathrm{mmol}, 1.0$ equiv, 26.2 $\mathrm{mg})$ and $\mathbf{2 f}(0.2 \mathrm{mmol}, 37.7 \mu \mathrm{L})$ and was purified by silica gel column chromatography (petroleum ether/EtOAc $=100 / 1 \sim 20 / 1)$ to obtain 3af as colorless oil $(22.5 \mathrm{mg}, 70 \%$ yield). ${ }^{1} \mathrm{H}$ NMR (400 MHz, $\left.\mathrm{CDCl}_{3}\right): \delta$ 7.23-7.19 (m, 2H), 7.17-7.12 (m, 2H), 6.93-6.88 (m, 3H), 6.82-6.80 (m, 1H), $6.39(\mathrm{~d}, J=15.7 \mathrm{~Hz}, 1 \mathrm{H}), 6.01(\mathrm{dt}, J=15.7,7.5 \mathrm{~Hz}, 1 \mathrm{H})$, $4.45(\mathrm{~d}, J=8.6 \mathrm{~Hz}, 1 \mathrm{H}), 4.15(\mathrm{~d}, J=8.6 \mathrm{~Hz}, 1 \mathrm{H}), 2.50(\mathrm{dd}, J=7.5,1.2 \mathrm{~Hz}, 2 \mathrm{H}), 1.40$ $(\mathrm{s}, 3 \mathrm{H}), 1.34(\mathrm{~s}, 9 \mathrm{H})$;

${ }^{13} \mathrm{C}$ NMR $\left(101 \mathrm{MHz}, \mathrm{CDCl}_{3}\right): \delta$ 159.5, 154.7, 134.9, 132.9, 132.5, 128.1, 126.6, 124.3, $124.2,122.9,120.5,109.7,81.9,78.6,45.6,44.2,28.8,25.0$;

HRMS: (ESI) calcd for $\mathrm{C}_{22} \mathrm{H}_{26} \mathrm{NaO}_{2}{ }^{+}[\mathrm{M}+\mathrm{Na}]^{+} 345.1825$; found 345.1825.

The enantiomeric purity was established by HPLC analysis using a chiral column: OD$\mathrm{H}$ column, $30{ }^{\circ} \mathrm{C}, n$-Hexane/i-Propanol $=97 / 3$ as eluent, $254 \mathrm{~nm}, 1 \mathrm{~mL} / \mathrm{min}$. $\mathrm{tR}=4.9$ $\min$ (minor), 5.7 min (major).

Optical Rotation: $[\alpha]_{D}^{28}-1.9\left(\mathrm{c} 0.5, \mathrm{CHCl}_{3}\right)$ for $95 \%$ ee.

Absolute stereochemistry was determined through analogy with 3ac. 

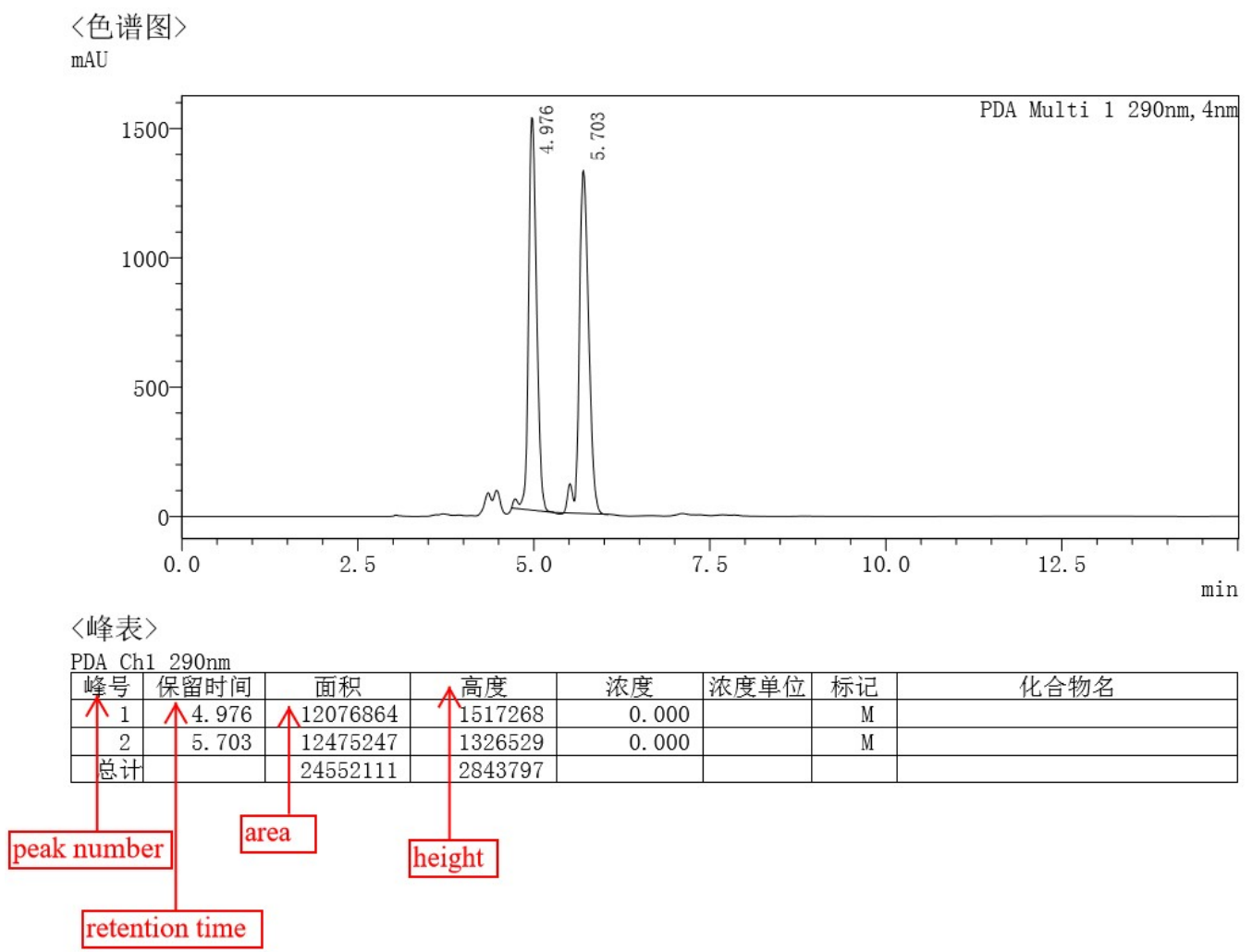

〈色谱图〉

$\mathrm{mAU}$

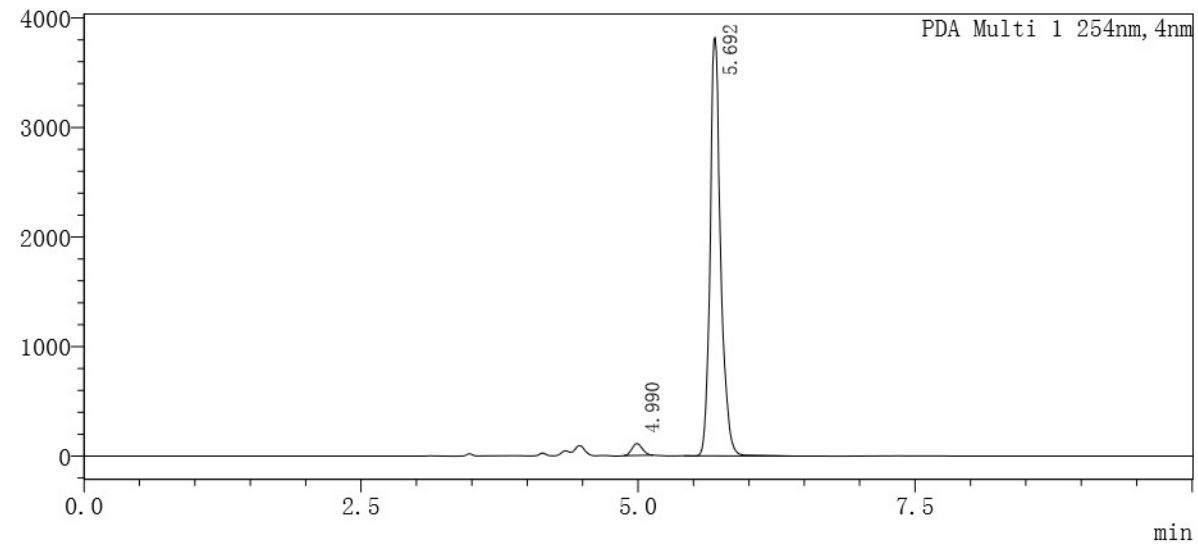

〈峰表〉

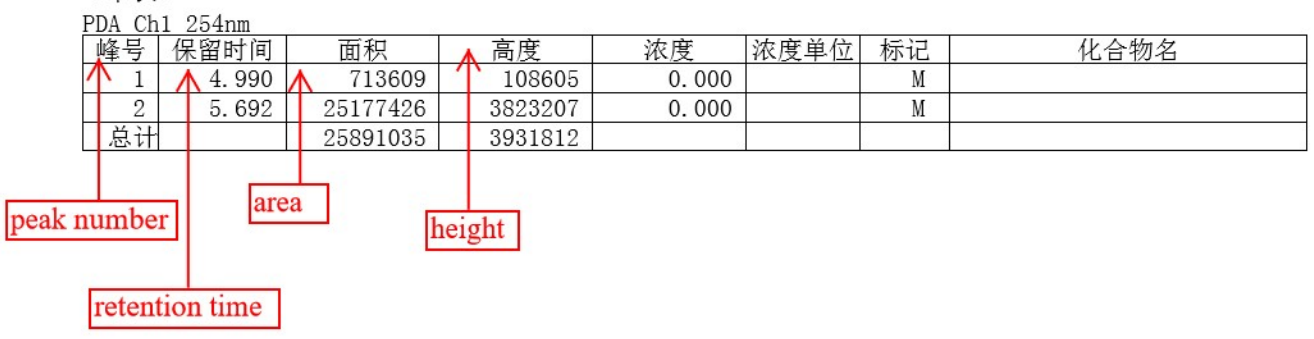


(R, E)-3-(3-(4-fluorophenyl)allyl)-3-methyl-2,3-dihydrobenzofuran (3ag)

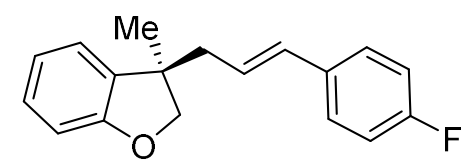

Chemical Formula: $\mathrm{C}_{18} \mathrm{H}_{17} \mathrm{FO}$

Exact Mass: 268.1263

3ag was prepared according to general procedure using $1 \mathrm{a}(0.1 \mathrm{mmol}, 1.0$ equiv, 26.2 $\mathrm{mg})$ and $\mathbf{2 g}(0.2 \mathrm{mmol}, 24 \mu \mathrm{L})$ and was purified by silica gel column chromatography (petroleum ether/EtOAc $=100 / 1 \sim 30 / 1)$ to obtain 3ag as colorless oil $(19.0 \mathrm{mg}, 71 \%$ yield). ${ }^{1} \mathrm{H}$ NMR $\left(400 \mathrm{MHz}, \mathrm{CDCl}_{3}\right): \delta$ 7.34-7.26 (m, 2H), 7.21-7.15 (m, 2H), 7.04-6.98 $(\mathrm{m}, 2 \mathrm{H}), 6.94(\mathrm{td}, J=7.4,1.0 \mathrm{~Hz}, 1 \mathrm{H}), 6.85(\mathrm{dt}, J=7.8,0.8 \mathrm{~Hz}, 1 \mathrm{H}), 6.40(\mathrm{dt}, J=15.7$, $1.4 \mathrm{~Hz}, 1 \mathrm{H}), 6.04(\mathrm{dt}, J=15.3,7.5 \mathrm{~Hz}, 1 \mathrm{H}), 4.47(\mathrm{~d}, J=8.6 \mathrm{~Hz}, 1 \mathrm{H}), 4.20(\mathrm{~d}, J=8.7$ $\mathrm{Hz}, 1 \mathrm{H}), 2.53$ (dd, $J=7.4,1.4 \mathrm{~Hz}, 2 \mathrm{H}), 1.44(\mathrm{~s}, 3 \mathrm{H})$;

${ }^{13} \mathrm{C} \mathrm{NMR}\left(101 \mathrm{MHz}, \mathrm{CDCl}_{3}\right): \delta 16(\mathrm{~d}, J=246.2 \mathrm{~Hz}), 159.5,134.8,133.5(\mathrm{~d}, J=3.3 \mathrm{~Hz})$, 132.2, 128.3, 127.6 (d, $J=7.9 \mathrm{~Hz}), 125.4$ (d, $J=2.2 \mathrm{~Hz}), 122.9,120.5,115.4$ (d, $J=$ $21.5 \mathrm{~Hz}), 109.7,81.9,45.7,44.3,25.0$;

${ }^{19} \mathrm{~F} \mathrm{NMR}\left(376 \mathrm{MHz}, \mathrm{CDCl}_{3}\right): \delta-114.95$;

HRMS: (APCI) calcd for $\mathrm{C}_{18} \mathrm{H}_{18} \mathrm{FO}+[\mathrm{M}+\mathrm{H}]^{+} 269.1337$; found 269.1347 .

The enantiomeric purity was established by HPLC analysis using a chiral column: OD$\mathrm{H}$ column, $30{ }^{\circ} \mathrm{C}, n$-Hexane/i-Propanol $=97 / 3$ as eluent, $254 \mathrm{~nm}, 1 \mathrm{~mL} / \mathrm{min}$. $\mathrm{tR}=4.7$ $\min$ (minor), 5.0 min (major).

Optical Rotation: $[\alpha]_{D}^{30}-18.1$ (c $\left.0.3, \mathrm{CHCl}_{3}\right)$ for $94 \%$ ee.

Absolute stereochemistry was determined through analogy with 3ac. 


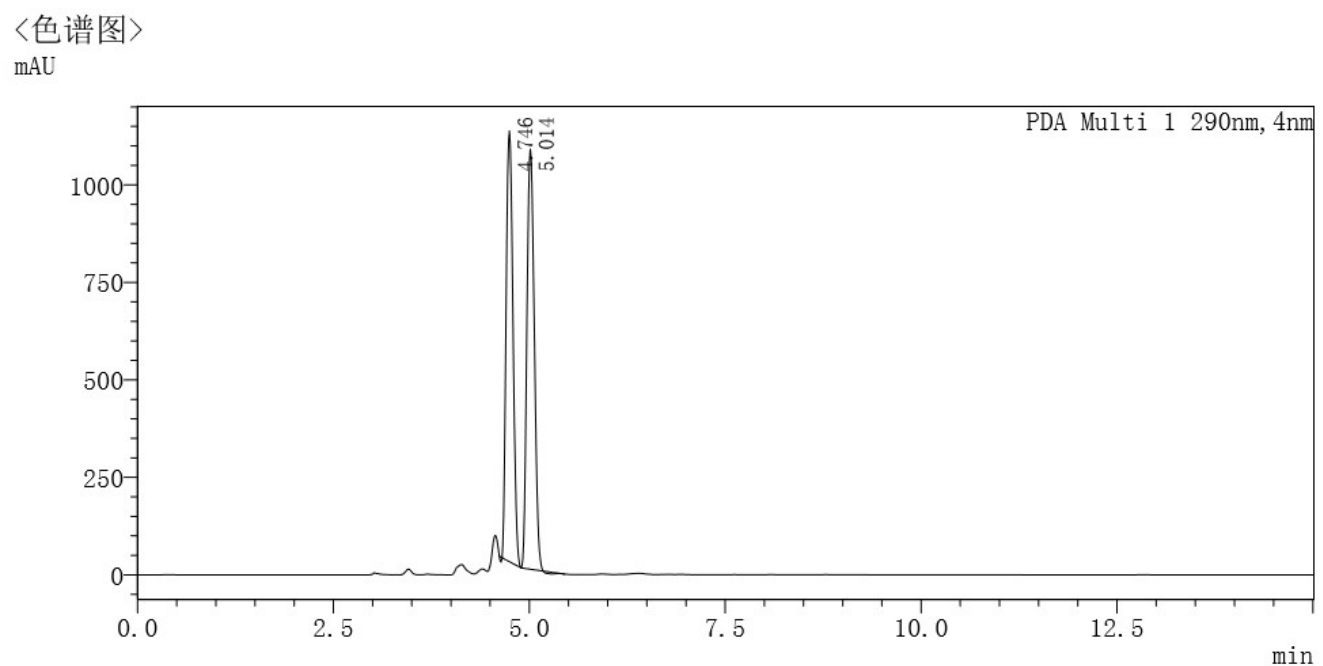

〈峰表〉

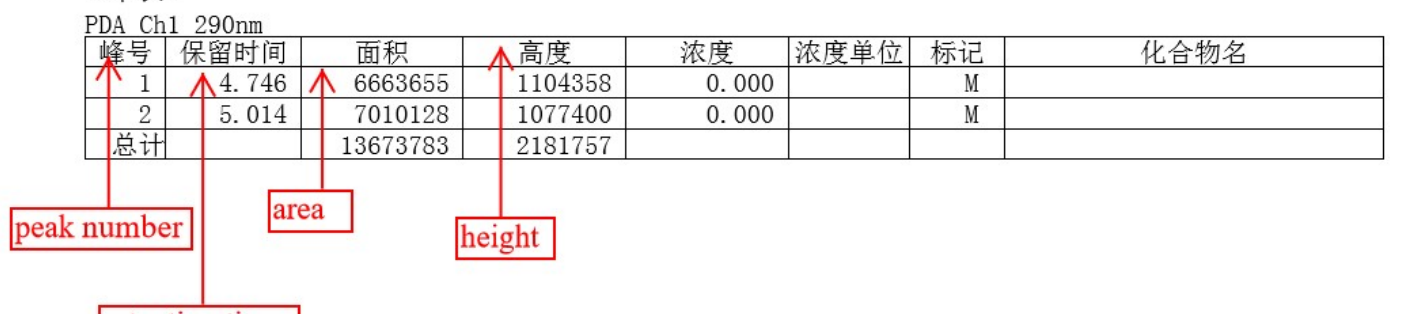

retention time

〈色谱图〉

$\mathrm{mAU}$

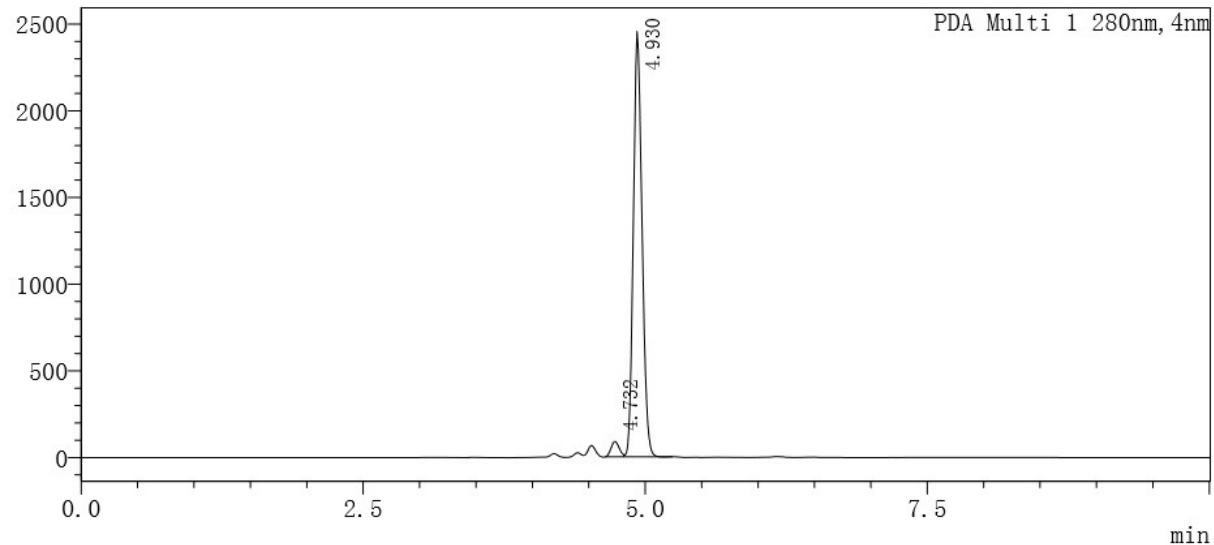

〈峰表〉

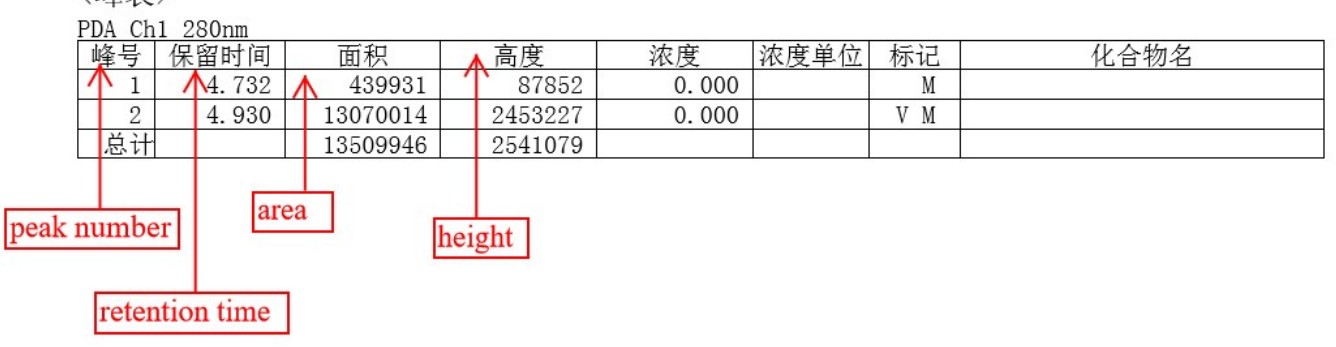


$(R, E)$-3-methyl-3-(3-(4-nitrophenyl)allyl)-2,3-dihydrobenzofuran (3ah)

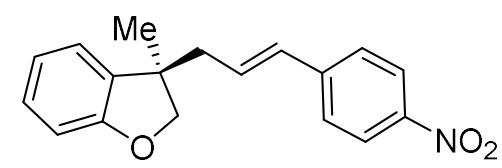

Chemical Formula: $\mathrm{C}_{18} \mathrm{H}_{17} \mathrm{NO}_{3}$

Exact Mass: 295.1208

3ah was prepared according to general procedure using $1 \mathrm{a}(0.1 \mathrm{mmol}, 1.0$ equiv, 26.2 $\mathrm{mg})$ and $\mathbf{2 h}(0.2 \mathrm{mmol}, 25.6 \mu \mathrm{L})$ and was purified by silica gel column chromatography (petroleum ether/EtOAc $=100 / 1 \sim 5 / 1)$ to obtain 3ah as colorless oil $(25.1 \mathrm{mg}, 85 \%$ yield). ${ }^{1} \mathrm{H}$ NMR (400 MHz, $\left.\mathrm{CDCl}_{3}\right): \delta$ 8.15-8.13 (m, 2H), 7.42-7.38 (m, 2H), 7.18-7.11 $(\mathrm{m}, 2 \mathrm{H}), 6.91(\mathrm{td}, J=7.4,0.9 \mathrm{~Hz}, 1 \mathrm{H}), 6.81(\mathrm{~d}, J=8.0 \mathrm{~Hz}, 1 \mathrm{H}), 6.45(\mathrm{~d}, J=15.8 \mathrm{~Hz}$, $1 \mathrm{H}), 6.31-6.23(\mathrm{~m}, 1 \mathrm{H}), 4.43(\mathrm{~d}, J=8.7 \mathrm{~Hz}, 1 \mathrm{H}), 4.18(\mathrm{~d}, J=8.7 \mathrm{~Hz}, 1 \mathrm{H}), 2.55(\mathrm{dd}, J=$ 7.5, $1.1 \mathrm{~Hz}, 2 \mathrm{H}), 1.43(\mathrm{~s}, 3 \mathrm{H})$;

${ }^{13} \mathrm{C}$ NMR $\left(101 \mathrm{MHz}, \mathrm{CDCl}_{3}\right): \delta$ 159.4, 146.6, 143.6, 134.1, 131.5, 131.1, 128.4, 126.6, $123.9,122.8,120.6,109.8,81.8,45.7,44.5,25.0$;

HRMS: (ESI) calcd for $\mathrm{C}_{18} \mathrm{H}_{17} \mathrm{NNaO}_{3}{ }^{+}[\mathrm{M}+\mathrm{Na}]^{+} 318.1101$; found 318.1104.

The enantiomeric purity was established by HPLC analysis using a chiral column: AD$\mathrm{H}$ column, $30{ }^{\circ} \mathrm{C}, n$-Hexane/i-Propanol $=95 / 5$ as eluent, $254 \mathrm{~nm}, 1 \mathrm{~mL} / \mathrm{min}$. $\mathrm{tR}=9.4$ $\min$ (minor), 9.9 min (major).

Optical Rotation: [a] $]^{28} 19.7$ (c $\left.0.4, \mathrm{CHCl}_{3}\right)$ for $90 \%$ ee. Absolute stereochemistry was determined through analogy with 3ac. 

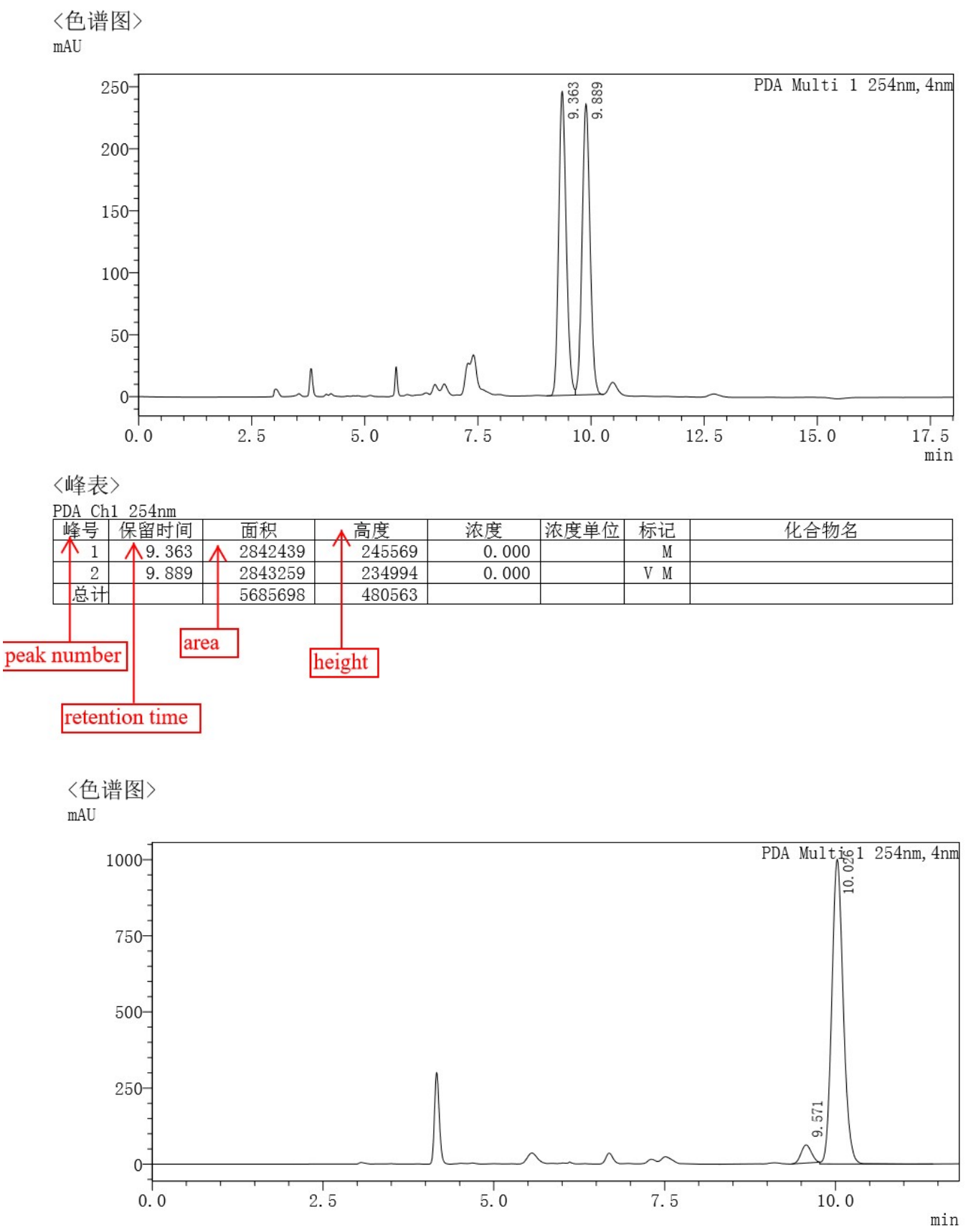

〈峰表〉

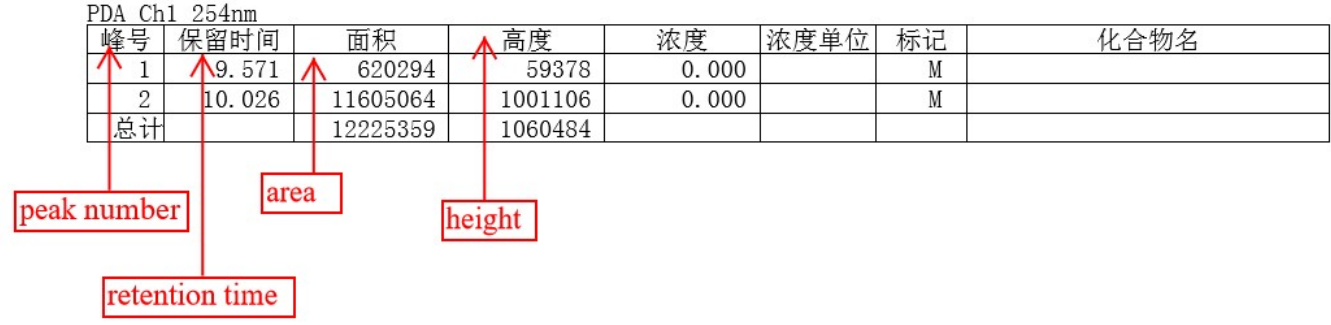


(R, E)-3-methyl-3-(3-(4-(trifluoromethyl)phenyl)allyl)-2,3-dihydrobenzofuran (3ai)

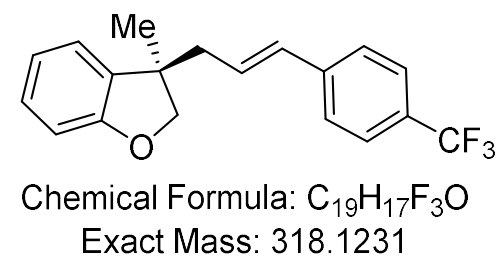

3ai was prepared according to general procedure using $1 \mathrm{a}(0.1 \mathrm{mmol}, 1.0$ equiv, 26.2 $\mathrm{mg})$ and $\mathbf{2 i}(0.2 \mathrm{mmol}, 30 \mu \mathrm{L})$ and was purified by silica gel column chromatography (petroleum ether/EtOAc $=100 / 1 \sim 30 / 1)$ to obtain 3ai as colorless oil $(20.0 \mathrm{mg}, 63 \%$ yield). ${ }^{1} \mathrm{H}$ NMR (400 MHz, $\left.\mathrm{CDCl}_{3}\right): \delta 7.54$ (d, $\left.J=8.2 \mathrm{~Hz}, 2 \mathrm{H}\right), 7.44-7.34(\mathrm{~m}, 2 \mathrm{H}), 7.22-$ $7.08(\mathrm{~m}, 2 \mathrm{H}), 6.91(\mathrm{td}, J=7.4,1.0 \mathrm{~Hz}, 1 \mathrm{H}), 6.82(\mathrm{ddd}, J=8.0,0.6 \mathrm{~Hz}, 1 \mathrm{H}), 6.43(\mathrm{~d}, J$ $=15.8 \mathrm{~Hz}, 1 \mathrm{H}), 6.28-6.13(\mathrm{~m}, 1 \mathrm{H}), 4.44(\mathrm{~d}, J=8.7 \mathrm{~Hz}, 1 \mathrm{H}), 4.17(\mathrm{~d}, J=8.7 \mathrm{~Hz}, 1 \mathrm{H})$, 2.54 (dd, J = 7.5, $1.2 \mathrm{~Hz}, 2 \mathrm{H}), 1.42(\mathrm{~s}, 3 \mathrm{H})$;

${ }^{13} \mathrm{C}$ NMR $\left(101 \mathrm{MHz}, \mathrm{CDCl}_{3}\right): \delta$ 159.5, 140.7, 134.5, 132.2, 129.2, 128.9, 128.6, 128.3, 126.2, 125.45 (q, $J=3.8 \mathrm{~Hz}), 122.9,120.6,109.8,81.9,45.6,44.3,25.0$;

${ }^{19} \mathrm{~F}$ NMR $\left(376 \mathrm{MHz}, \mathrm{CDCl}_{3}\right): \delta-62.35$;

HRMS: (APCl) calcd for $\mathrm{C}_{19} \mathrm{H}_{18} \mathrm{~F}_{3} \mathrm{O}^{+}[\mathrm{M}+\mathrm{H}]^{+} 319.1304$; found 319.1298.

The enantiomeric purity was established by HPLC analysis using a chiral column: OJ$\mathrm{H}$ column, $30{ }^{\circ} \mathrm{C}, n$-Hexane/i-Propanol $=95 / 5$ as eluent, $254 \mathrm{~nm}, 1 \mathrm{~mL} / \mathrm{min}$. $\mathrm{tR}=8.8$ $\min$ (major), $9.4 \min$ (minor).

Optical Rotation: $[\alpha]_{\mathrm{D}}{ }^{30}-12.5$ (c $\left.0.3, \mathrm{CHCl}_{3}\right)$ for $93 \%$ ee. Absolute stereochemistry was determined through analogy with 3ac. 


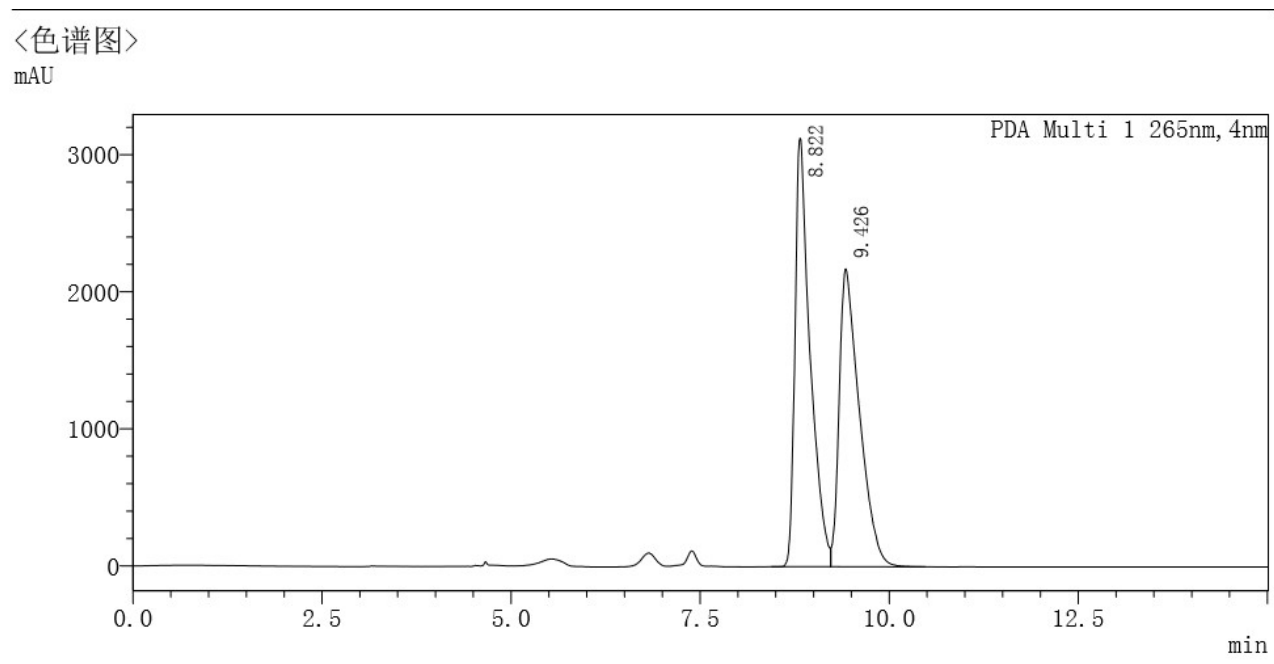

〈峰表〉

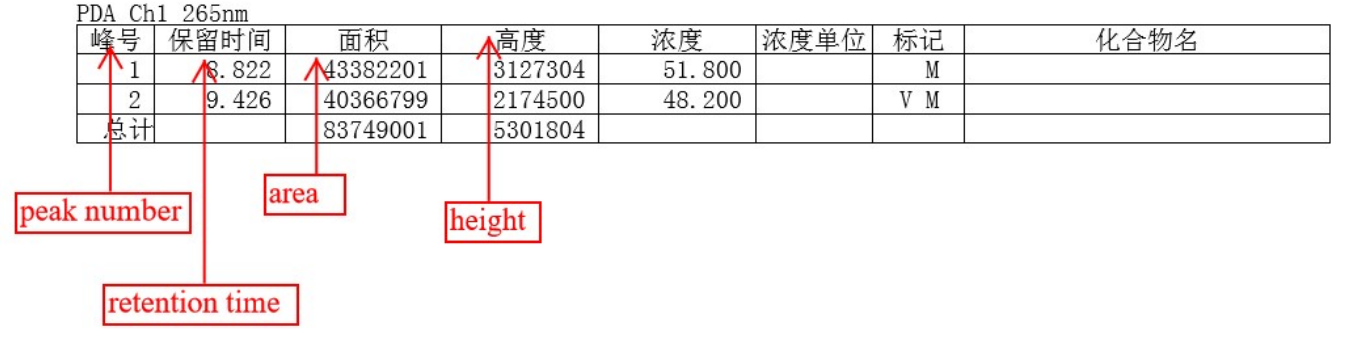

〈色谱图〉

mAU

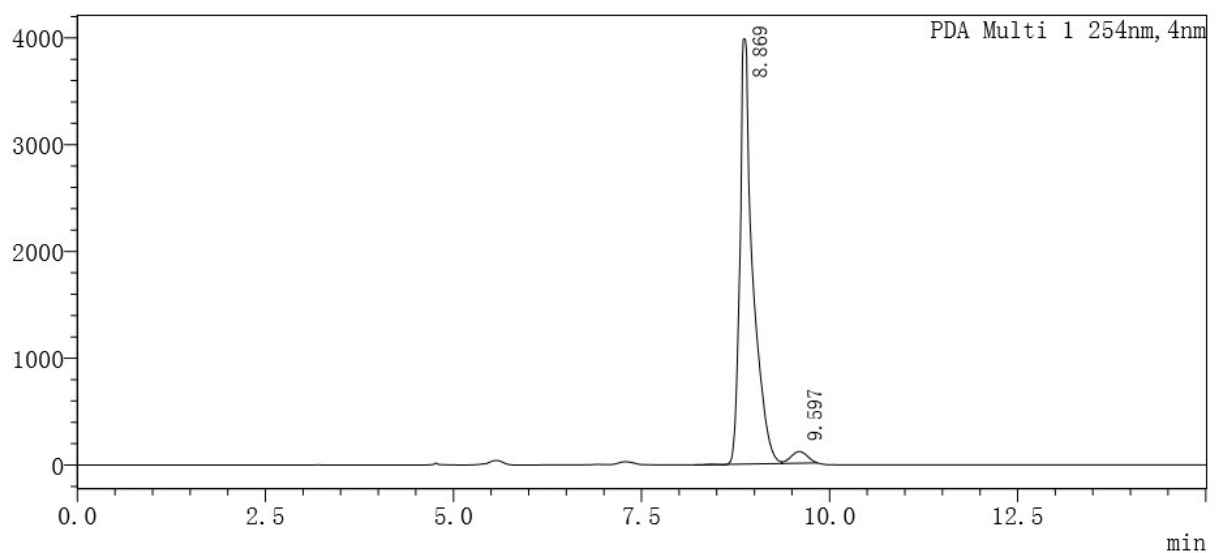

〈峰表〉

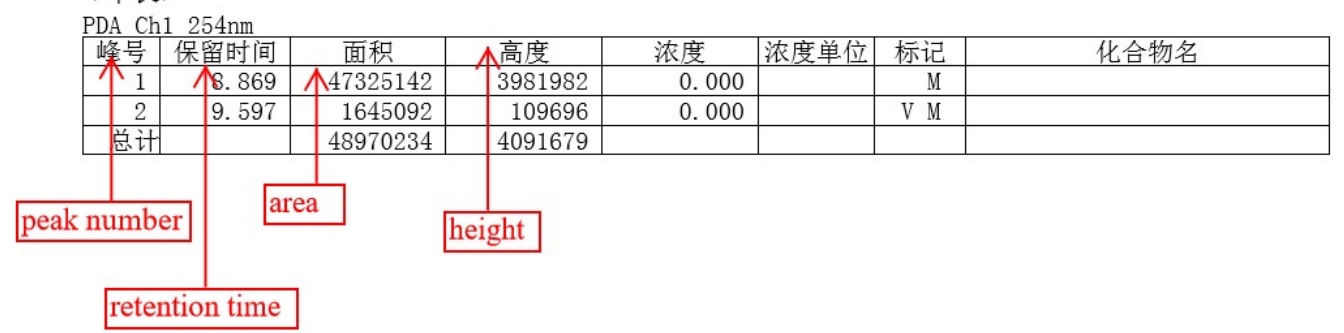


(R, E)-3-(3-([1,1'-biphenyl]-4-yl)allyl)-3-methyl-2,3-dihydrobenzofuran (3aj)

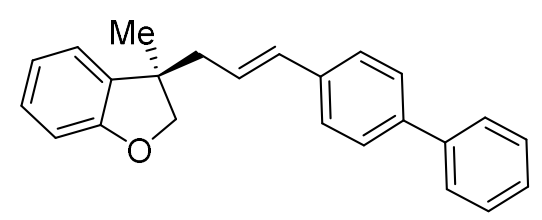

Chemical Formula: $\mathrm{C}_{24} \mathrm{H}_{22} \mathrm{O}$

Exact Mass: 326.1671

3aj was prepared according to general procedure using 1 a $(0.1 \mathrm{mmol}, 1.0$ equiv, 26.2 $\mathrm{mg}$ ) and $2 \mathrm{j}(0.2 \mathrm{mmol}, 36 \mathrm{mg})$ and was purified by silica gel column chromatography (petroleum ether/EtOAc $=100 / 1 \sim 30 / 1)$ to obtain 3aj as colorless oil $(28.0 \mathrm{mg}, 86 \%$ yield). ${ }^{1} \mathrm{H}$ NMR (400 MHz, $\left.\mathrm{CDCl}_{3}\right): \delta$ 7.71-7.52 (m, 4H), 7.48-7.31 (m, 5H), 7.23-7.11 (m, 2H), 6.92 (td, $J=7.4,1.0 \mathrm{~Hz}, 1 \mathrm{H}), 6.83(\mathrm{dt}, J=7.8,0.9 \mathrm{~Hz}, 1 \mathrm{H}), 6.58-6.39(\mathrm{~m}, 1 \mathrm{H})$, 6.17 (dt, $J=15.8,7.5 \mathrm{~Hz}, 1 \mathrm{H}), 4.47(\mathrm{~d}, J=8.6 \mathrm{~Hz}, 1 \mathrm{H}), 4.18$ (d, J = 8.6 Hz, 1H), 2.55 (dd, $J=7.5,1.3 \mathrm{~Hz}, 2 \mathrm{H}), 1.43(\mathrm{~s}, 3 \mathrm{H})$;

${ }^{13} \mathrm{C} \mathrm{NMR}\left(101 \mathrm{MHz}, \mathrm{CDCl}_{3}\right): \delta 159.5,140.7,140.0,136.3,134.8,133.0,128.8,128.2$, $127.2,126.9,126.5,125.8,122.9,120.5,109.7,82.0,45.7,44.3,25.0$;

HRMS: (APCl) calcd for $\mathrm{C}_{24} \mathrm{H}_{23} \mathrm{O}^{+}[\mathrm{M}+\mathrm{H}]^{+} 327.1743$; found 327.1739 .

The enantiomeric purity was established by HPLC analysis using a chiral column: OD$\mathrm{H}$ column, $30{ }^{\circ} \mathrm{C}, n$-Hexane/i-Propanol $=98 / 2$ as eluent, $254 \mathrm{~nm}, 0.5 \mathrm{~mL} / \mathrm{min}$. tR $=6$ $\min$ (minor), 9 min (major).

Optical Rotation: $[\alpha]_{\mathrm{D}}{ }^{28} 36.8$ (c $\left.0.1, \mathrm{CHCl}_{3}\right)$ for $93 \%$ ee.

Absolute stereochemistry was determined through analogy with 3ac. 

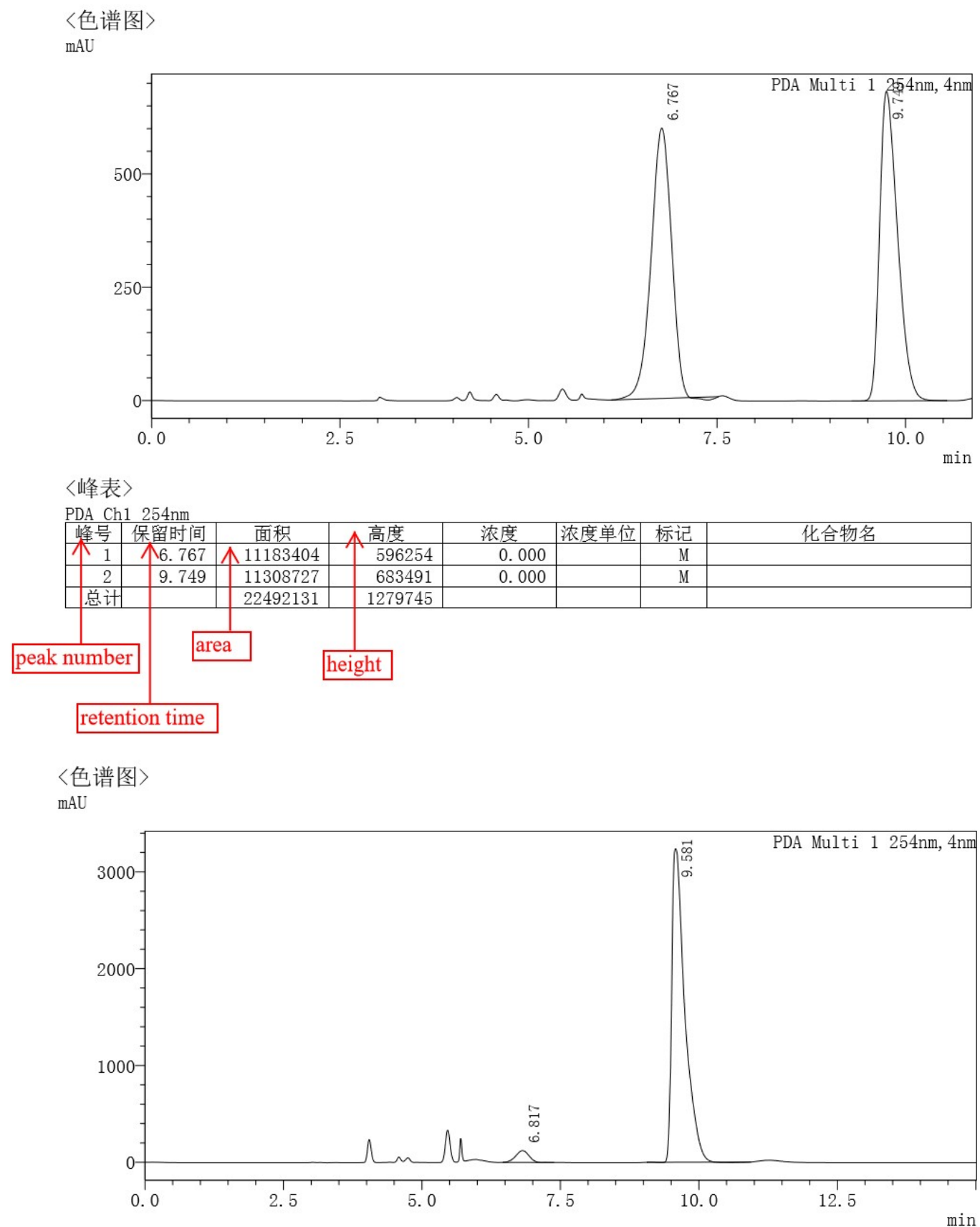

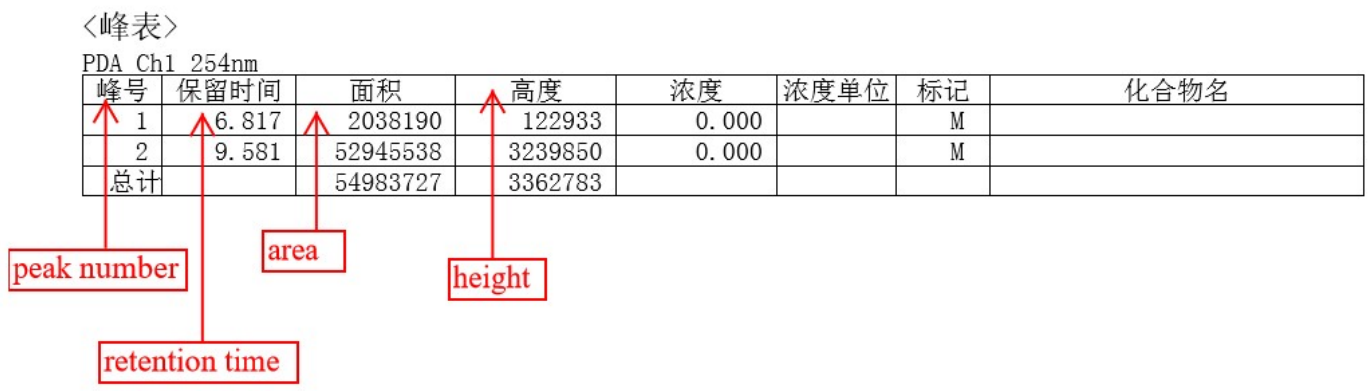


(R, E)-3-(3-(4-bromophenyl)allyl)-3-methyl-2,3-dihydrobenzofuran (3ak)

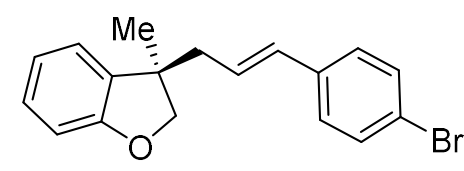

Chemical Formula: $\mathrm{C}_{18} \mathrm{H}_{17} \mathrm{BrO}$

Exact Mass: 328.0463

3ak was prepared according to general procedure using 1 a $(0.1 \mathrm{mmol}, 1.0$ equiv, 26.2 $\mathrm{mg})$ and $\mathbf{2 k}(0.2 \mathrm{mmol}, 26 \mu \mathrm{L})$ and was purified by silica gel column chromatography (petroleum ether/EtOAc $=100 / 1 \sim 30 / 1)$ to obtain 3ak as colorless oil $(29.0 \mathrm{mg}, 88 \%$ yield). ${ }^{1} \mathrm{H}$ NMR $\left(400 \mathrm{MHz}, \mathrm{CDCl}_{3}\right): \delta$ 7.48-7.38 (m, 2H), 7.22-7.10 (m, 4H), 6.90 (td, J $=7.4,1.0 \mathrm{~Hz}, 1 \mathrm{H}), 6.85-6.79(\mathrm{~m}, 1 \mathrm{H}), 6.43-6.27(\mathrm{~m}, 1 \mathrm{H}), 6.09(\mathrm{dt}, J=15.8,7.5 \mathrm{~Hz}, 1 \mathrm{H})$, $4.43(\mathrm{~d}, J=8.7 \mathrm{~Hz}, 1 \mathrm{H}), 4.16(\mathrm{~d}, J=8.7 \mathrm{~Hz}, 1 \mathrm{H}), 2.50$ (dd, J = 7.5, $1.3 \mathrm{~Hz}, 2 \mathrm{H}), 1.40$ $(\mathrm{s}, 3 \mathrm{H})$;

${ }^{13} \mathrm{C}$ NMR $\left(101 \mathrm{MHz}, \mathrm{CDCl}_{3}\right): \delta$ 159.4, 136.2, 134.6, 132.2, 131.5, 128.2, 127.6, 126.5, $122.9,120.9,120.5,109.7,81.9,45.6,44.3,24.9$;

HRMS: (APCI) calcd for $\mathrm{C}_{18} \mathrm{H}_{18} \mathrm{BrO}^{+}[\mathrm{M}+\mathrm{H}]^{+} 329.0536$; found 329.0543 .

The enantiomeric purity was established by HPLC analysis using a chiral column: OD$\mathrm{H}$ column, $30{ }^{\circ} \mathrm{C}, n$-Hexane $/ \mathrm{i}$-Propanol $=97 / 3$ as eluent, $254 \mathrm{~nm}, 1 \mathrm{~mL} / \mathrm{min}$. $\mathrm{tR}=5.0$ $\min$ (minor), $5.5 \mathrm{~min}$ (major).

Optical Rotation: $[\alpha]_{\mathrm{D}}^{30}-1$ (c $\left.0.3, \mathrm{CHCl}_{3}\right)$ for $94 \%$ ee.

Absolute stereochemistry was determined through analogy with 3ac. 


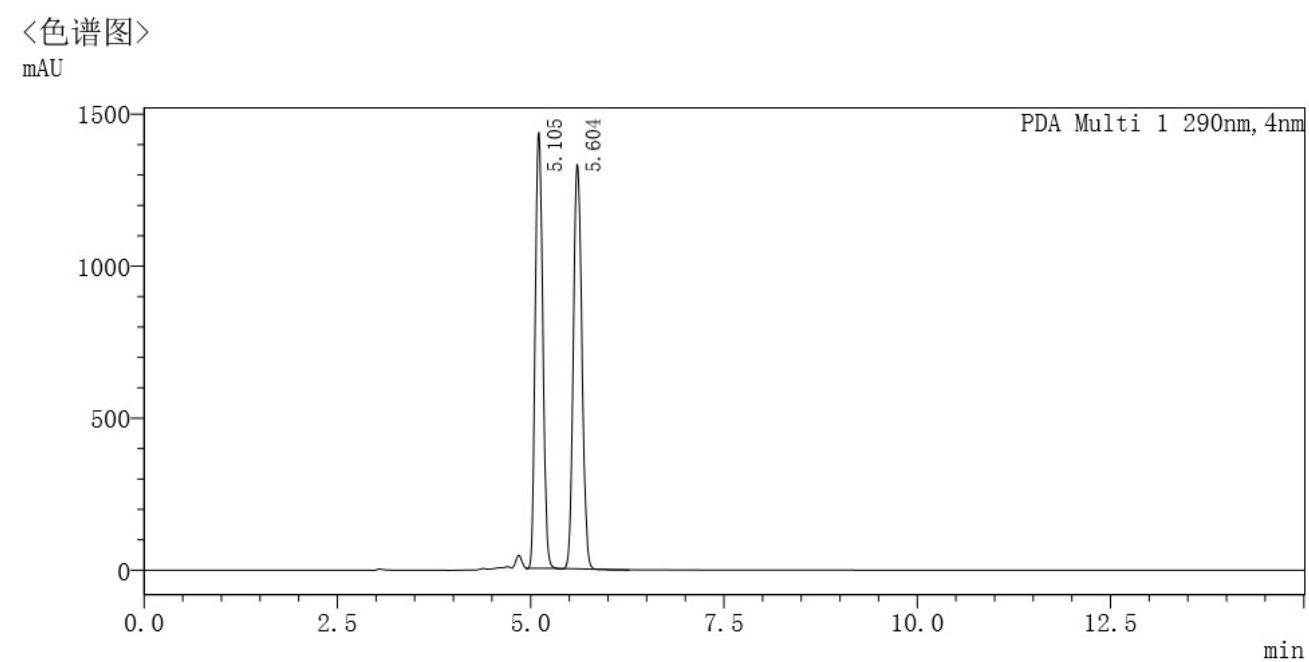

〈峰表〉

\begin{tabular}{|c|c|c|c|c|c|c|c|}
\hline 峰号 & 保留时间 & 面积 & 高度 & 浓度 & 浓度单位 & 标记 & 化合物名 \\
\hline 梠 1 & 木5.105 & 19694000 & 个 1434717 & 0.000 & & M & \\
\hline 2 & 5.604 & 10235645 & 1330338 & 0.000 & & M & \\
\hline 总计 & & 19929644 & 2765055 & & & & \\
\hline
\end{tabular}

retention time

〈色谱图〉

$\mathrm{mAU}$

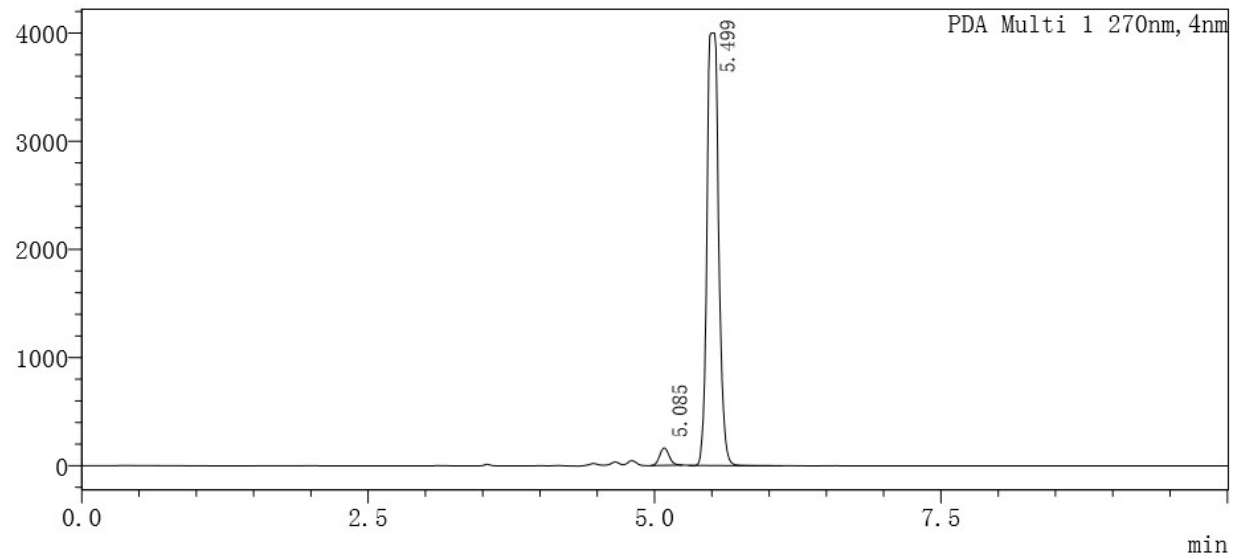

〈峰表〉

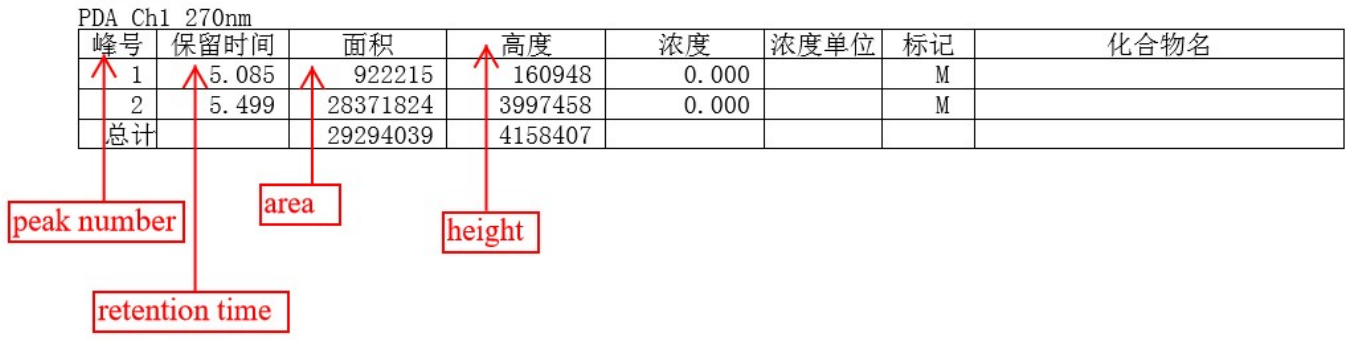


Methyl ( $R, E)$-4-(3-(3-methyl-2,3-dihydrobenzofuran-3-yl)prop-1-en-1-yl)benzoate (3al)

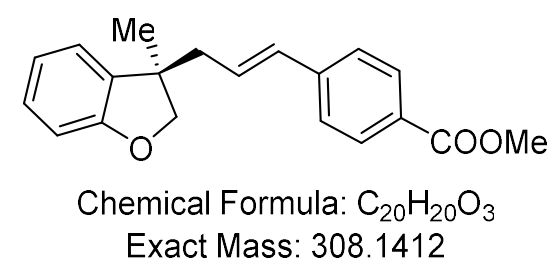

3al was prepared according to general procedure using $1 \mathrm{a}(0.1 \mathrm{mmol}, 1.0$ equiv, 26.2 $\mathrm{mg}$ ) and $2 \mathrm{I}(0.2 \mathrm{mmol}, 32.4 \mathrm{mg})$ and was purified by silica gel column chromatography (petroleum ether/EtOAc $=100 / 1 \sim 30 / 1)$ to obtain 3 al as colorless oil $(21.2 \mathrm{mg}, 69 \%$ yield). ${ }^{1} \mathrm{H}$ NMR (400 MHz, $\left.\mathrm{CDCl}_{3}\right): \delta 7.96(\mathrm{~d}, J=8.4 \mathrm{~Hz}, 2 \mathrm{H}), 7.35(\mathrm{~d}, J=8.3 \mathrm{~Hz}, 2 \mathrm{H})$, 7.17-7.08 (m, 2H), $6.90(\mathrm{td}, J=7.4,1.0 \mathrm{~Hz}, 1 \mathrm{H}), 6.85-6.78(\mathrm{~m}, 1 \mathrm{H}), 6.52-6.38(\mathrm{~m}, 1 \mathrm{H})$, 6.31-6.15 (m, 1H), $4.44(\mathrm{~d}, J=8.7 \mathrm{~Hz}, 1 \mathrm{H}), 4.17(\mathrm{~d}, J=8.6 \mathrm{~Hz}, 1 \mathrm{H}), 3.91(\mathrm{~s}, 3 \mathrm{H}), 2.53$ (dd, $J=7.5,1.3 \mathrm{~Hz}, 2 \mathrm{H}), 1.42(\mathrm{~s}, 3 \mathrm{H})$;

${ }^{13} \mathrm{C}$ NMR NMR $\left(101 \mathrm{MHz}, \mathrm{CDCl}_{3}\right): \delta 166.9,159.5,141.7,134.5,132.6,129.9,128.7$, 128.6, 128.3, 125.9, 122.9, 120.5, 109.8, 81.9, 52.0, 45.6, 44.4, 25.0;

HRMS: (ESI) calcd for $\mathrm{C}_{20} \mathrm{H}_{20} \mathrm{NaO}_{3}{ }^{+}[\mathrm{M}+\mathrm{Na}]^{+} 331.1305$; found 331.1310 .

The enantiomeric purity was established by HPLC analysis using a chiral column: OD$\mathrm{H}$ column, $30{ }^{\circ} \mathrm{C}, n$-Hexane/i-Propanol $=85 / 15$ as eluent, $254 \mathrm{~nm}, 1 \mathrm{~mL} / \mathrm{min}$. $\mathrm{tR}=5.5$ $\min (\operatorname{minor}), 5.8 \mathrm{~min}$ (major).

Optical Rotation: $[\alpha]_{\mathrm{D}}{ }^{28} 13.9$ (c $\left.0.3, \mathrm{CHCl}_{3}\right)$ for $93 \%$ ee.

Absolute stereochemistry was determined through analogy with 3ac. 
〈色谱图〉

$\mathrm{mAU}$

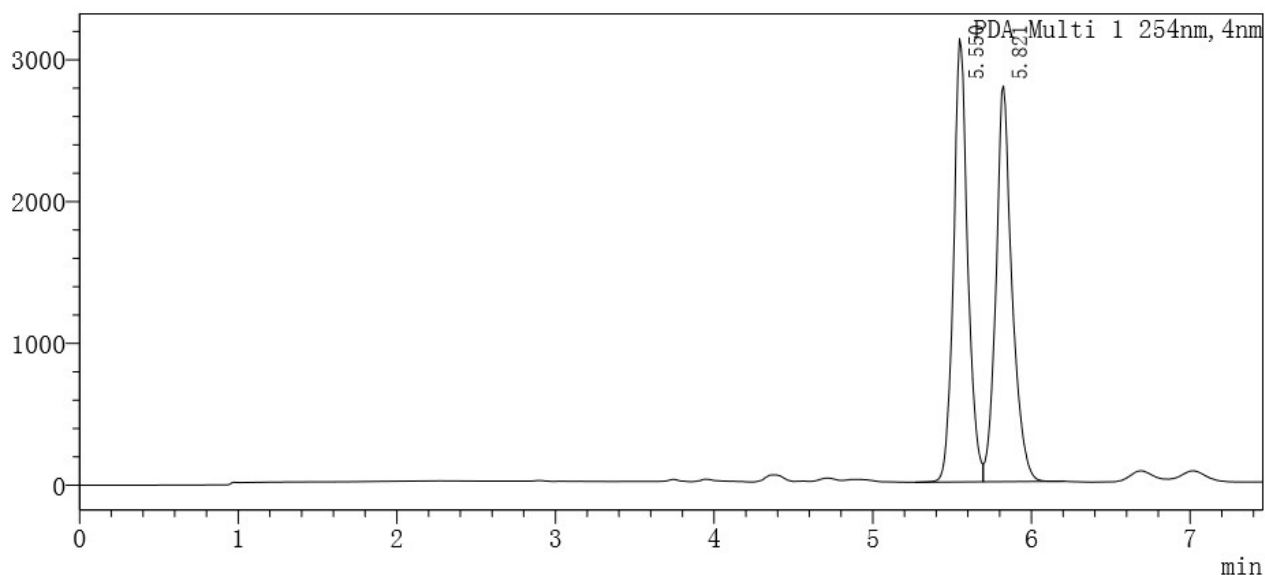

〈峰表〉

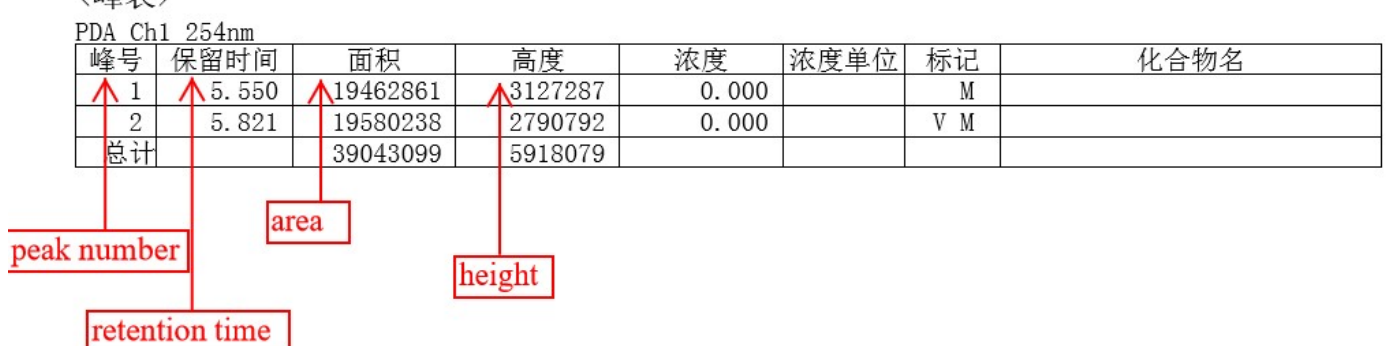

retention time

〈色谱图〉

$\mathrm{mAU}$

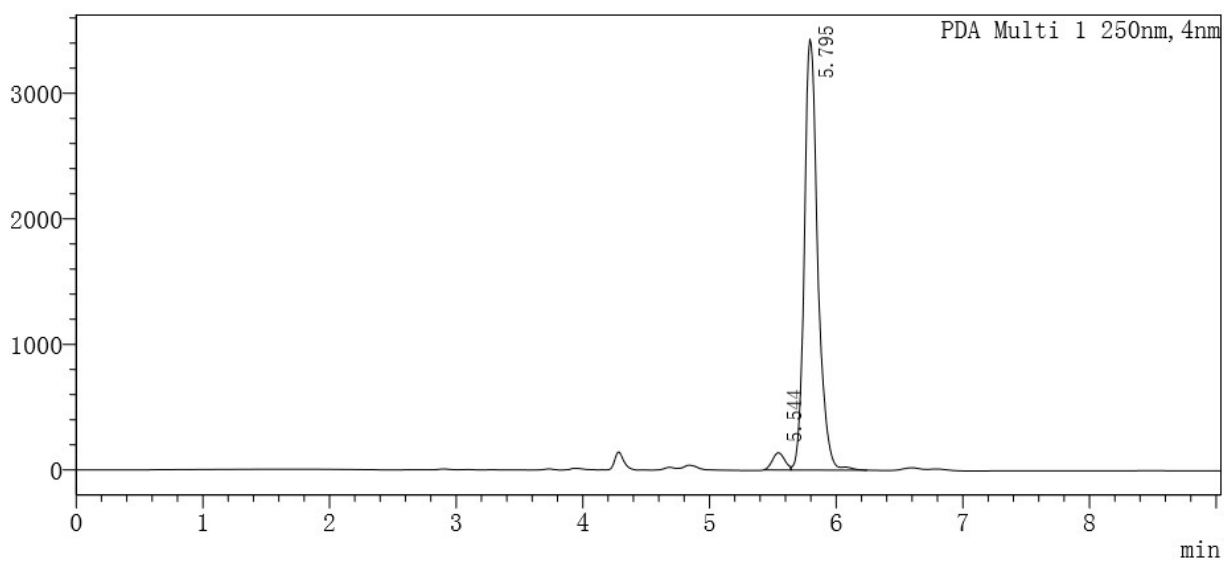

〈峰表〉

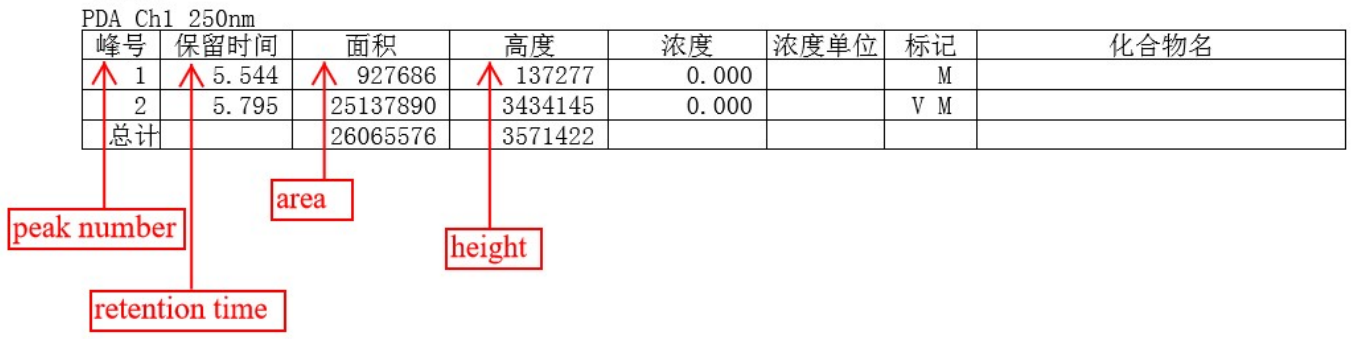


(R, E)-3-methyl-3-(3-(3-(trifluoromethoxy)phenyl)allyl)-2,3-dihydrobenzofuran (3am)

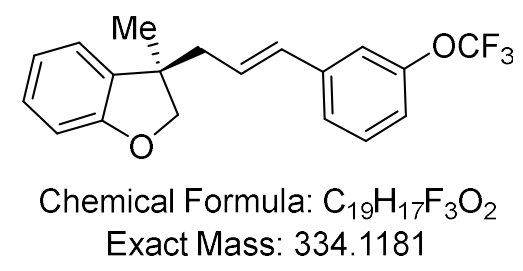

3am was prepared according to general procedure using 1a $(0.1 \mathrm{mmol}, 1.0$ equiv, 26.2 $\mathrm{mg})$ and $2 \mathrm{~m}(0.2 \mathrm{mmol}, 37.6 \mathrm{mg})$ and was purified by silica gel column chromatography (petroleum ether/EtOAc $=100 / 1 \sim 30 / 1)$ to obtain 3am as colorless oil $(17.4 \mathrm{mg}, 52 \%$ yield). ${ }^{1} \mathrm{H}$ NMR $\left(400 \mathrm{MHz}, \mathrm{CDCl}_{3}\right): \delta 7.30(\mathrm{t}, J=7.9 \mathrm{~Hz}, 1 \mathrm{H}), 7.25-7.20(\mathrm{~m}, 1 \mathrm{H}), 7.19-$ $7.10(\mathrm{~m}, 3 \mathrm{H}), 7.09-7.04(\mathrm{~m}, 1 \mathrm{H}), 6.91(\mathrm{td}, J=7.5,1.0 \mathrm{~Hz}, 1 \mathrm{H}), 6.81(\mathrm{dt}, J=8.0,0.8 \mathrm{~Hz}$, $1 \mathrm{H}), 6.54-6.30(\mathrm{~m}, 1 \mathrm{H}), 6.13(\mathrm{dt}, J=15.7,7.5 \mathrm{~Hz}, 1 \mathrm{H}), 4.44(\mathrm{~d}, J=8.7 \mathrm{~Hz}, 1 \mathrm{H}), 4.17$ (d, $J=8.7 \mathrm{~Hz}, 1 \mathrm{H}), 2.52(\mathrm{dd}, J=7.5,1.3 \mathrm{~Hz}, 2 \mathrm{H}), 1.41(\mathrm{~s}, 3 \mathrm{H})$;

${ }^{13} \mathrm{C}$ NMR $\left(101 \mathrm{MHz}, \mathrm{CDCl}_{3}\right): \delta 159.5,149.6,139.5,134.6,13,129.8,128.3,127.7$, 124.4, 122.9, 120.6, 120.0 (q, J = 115.1 Hz), 119.4, 118.5, 109.8, 81.9, 45.6, 44.2, 24.9; ${ }^{19} \mathrm{~F}$ NMR $\left(376 \mathrm{MHz}, \mathrm{CDCl}_{3}\right): \delta-57.58$;

HRMS: (ESI) calcd for $\mathrm{C}_{19} \mathrm{H}_{17} \mathrm{~F}_{3} \mathrm{NaO}_{2}{ }^{+}[\mathrm{M}+\mathrm{Na}]^{+}$357.1073; found 357.1072.

The enantiomeric purity was established by HPLC analysis using a chiral column: OJ$\mathrm{H}$ column, $30{ }^{\circ} \mathrm{C}, n$-Hexane/i-Propanol $=95 / 5$ as eluent, $254 \mathrm{~nm}, 1 \mathrm{~mL} / \mathrm{min} . \mathrm{tR}=5.7$ $\min$ (minor), $6.5 \mathrm{~min}$ (major).

Optical Rotation: $[\alpha]_{\mathrm{D}}{ }^{28} 39.2$ (c $0.1, \mathrm{CHCl}_{3}$ ) for $97 \%$ ee. Absolute stereochemistry was determined through analogy with 3ac. 
〈色谱图〉

$\mathrm{mAU}$
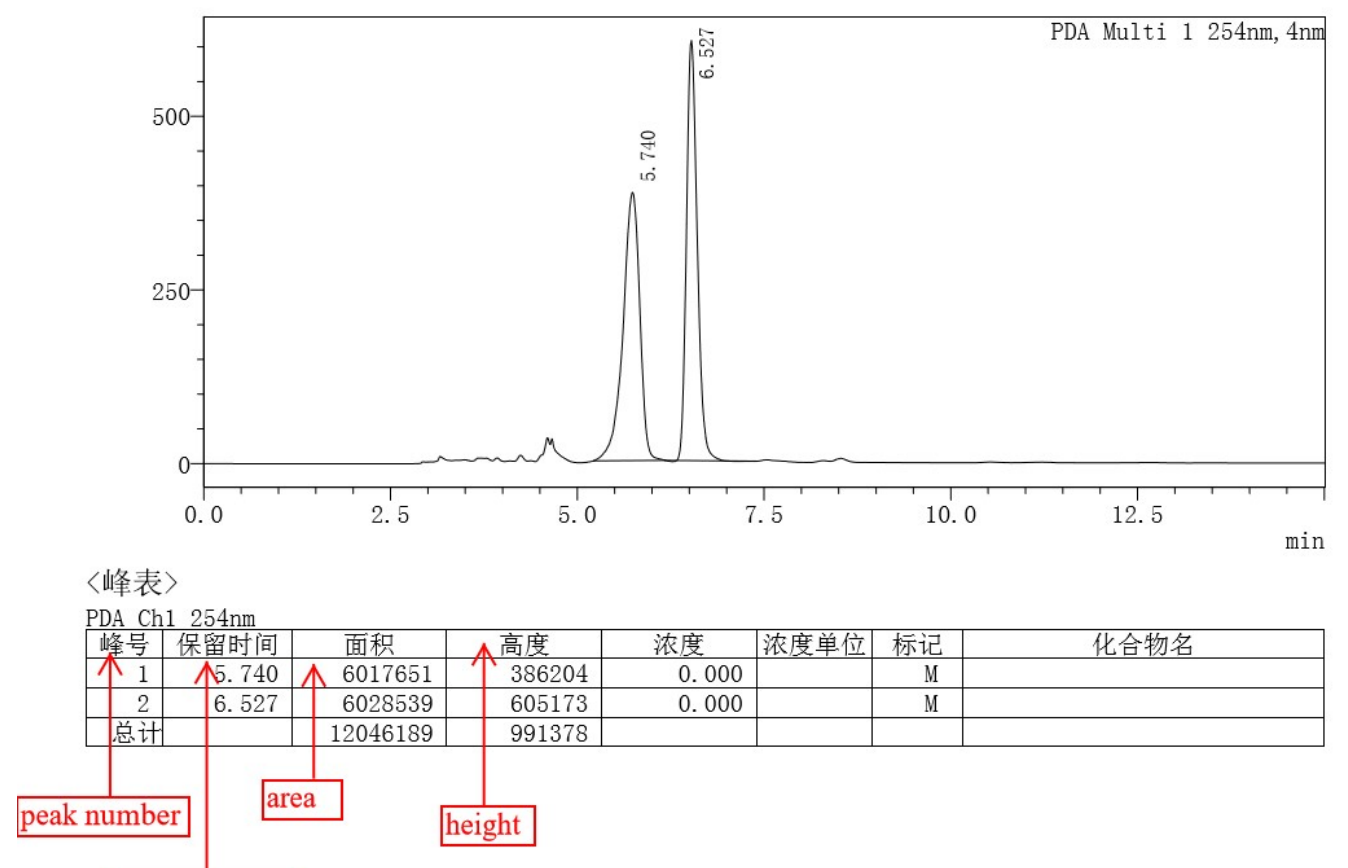

retention time

〈色谱图〉

$\mathrm{mAU}$

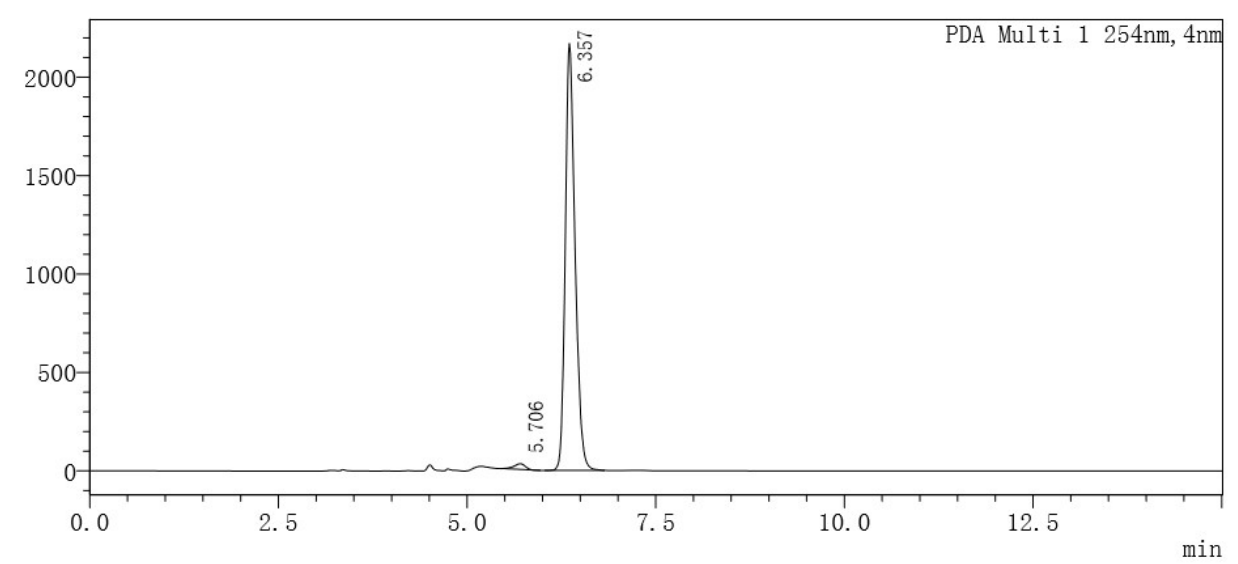

〈峰表〉

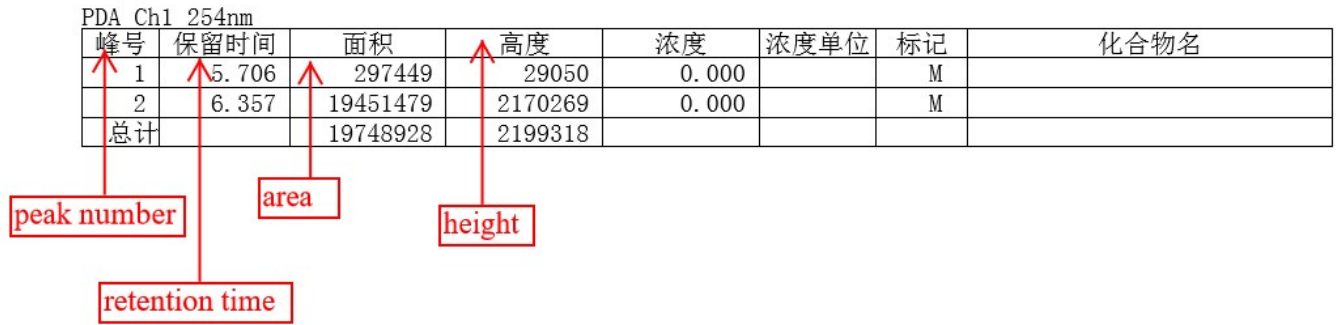


( $R, E)$-3-methyl-3-(3-(3-phenoxyphenyl)allyl)-2,3-dihydrobenzofuran (3an)

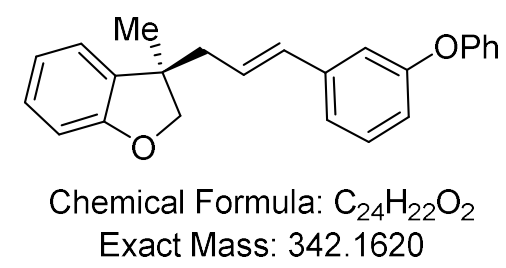

3an was prepared according to general procedure using 1 a $(0.1 \mathrm{mmol}, 1.0$ equiv, 26.2 $\mathrm{mg})$ and $2 \mathrm{n}(0.2 \mathrm{mmol}, 39 \mathrm{mg})$ and was purified by silica gel column chromatography (petroleum ether/EtOAc $=100 / 1 \sim 20 / 1)$ to obtain 3an as colorless oil $(28.4 \mathrm{mg}, 83 \%$ yield). ${ }^{1} \mathrm{H}$ NMR (400 MHz, $\left.\mathrm{CDCl}_{3}\right): \delta$ 7.38-7.31 (m, 2H), 7.29-7.21 (m, 1H), 7.17-7.08 (m, 3H), 7.08-6.94 (m, 4H), 6.92-6.83 (m, 2H), $6.80(\mathrm{dt}, J=8.0,0.8 \mathrm{~Hz}, 1 \mathrm{H}), 6.37(\mathrm{dt}$, $J=15.7,1.3 \mathrm{~Hz}, 1 \mathrm{H}), 6.09(\mathrm{dt}, J=15.7,7.5 \mathrm{~Hz}, 1 \mathrm{H}), 4.42(\mathrm{~d}, J=8.6 \mathrm{~Hz}, 1 \mathrm{H}), 4.15(\mathrm{~d}$, $J=8.7 \mathrm{~Hz}, 1 \mathrm{H}), 2.49(\mathrm{dd}, J=7.5,1.4 \mathrm{~Hz}, 2 \mathrm{H}), 1.39(\mathrm{~s}, 3 \mathrm{H})$;

${ }^{13} \mathrm{C}$ NMR NMR (101 MHz, $\left.\mathrm{CDCl}_{3}\right): \delta 159.5,157.4,157.2,139.3,134.7,132.8,129.7$ $(\mathrm{d}, J=1.9 \mathrm{~Hz}), 128.2,126.6,123.2,122.9,121.2,120.5,118.8,117.6,116.5,109.7$, 82.0, 45.6, 44.2, 25.0;

HRMS: (ESI) calcd for $\mathrm{C}_{24} \mathrm{H}_{23} \mathrm{O}_{2}{ }^{+}[\mathrm{M}+\mathrm{H}]^{+} 343.1693$; found 343.1686.

The enantiomeric purity was established by HPLC analysis using a chiral column: OD$\mathrm{H}$ column, $30{ }^{\circ} \mathrm{C}, n$-Hexane/i-Propanol $=85 / 15$ as eluent, $254 \mathrm{~nm}, 1 \mathrm{~mL} / \mathrm{min} . \mathrm{tR}=7$ $\min$ (minor), $13 \mathrm{~min}$ (major).

Optical Rotation: $[\alpha]_{\mathrm{D}}^{28} 6.5$ (c $\left.0.6, \mathrm{CHCl}_{3}\right)$ for $93 \%$ ee.

Absolute stereochemistry was determined through analogy with 3ac. 
〈色谱图〉

mAU

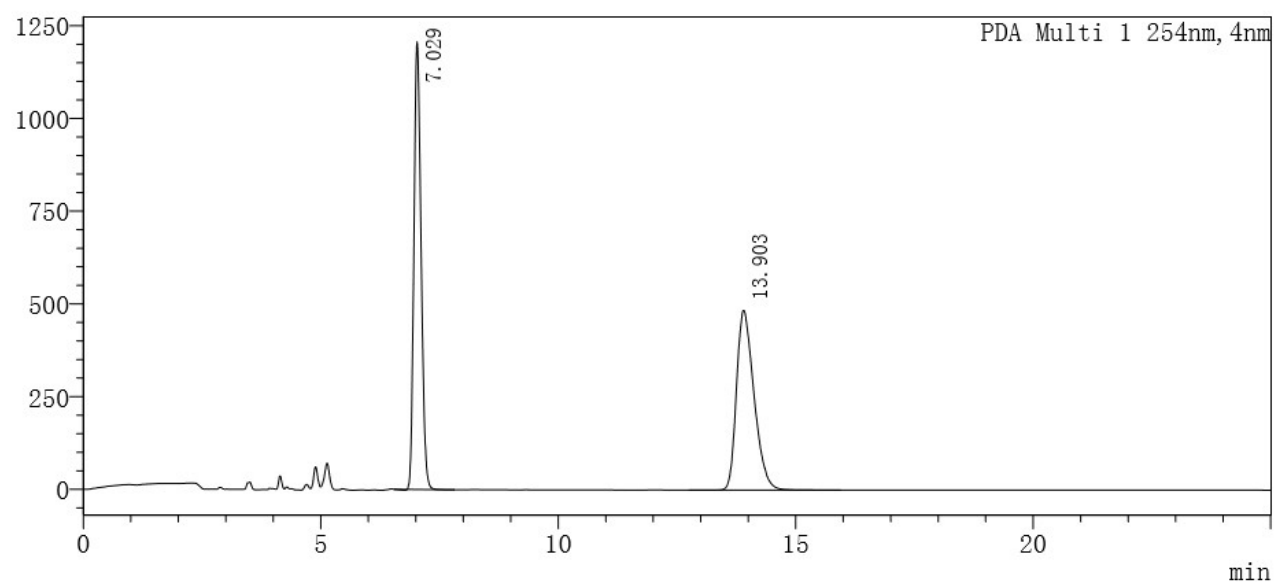

〈峰表〉

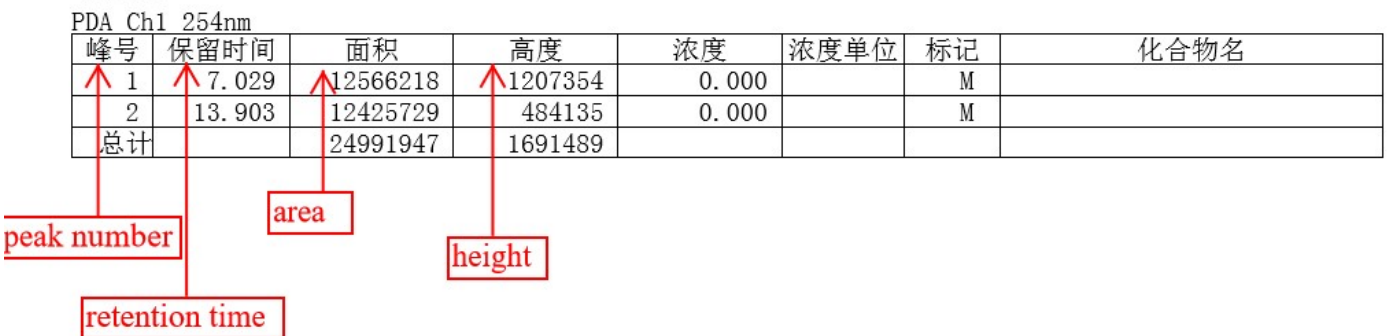

〈色谱图〉

mAU

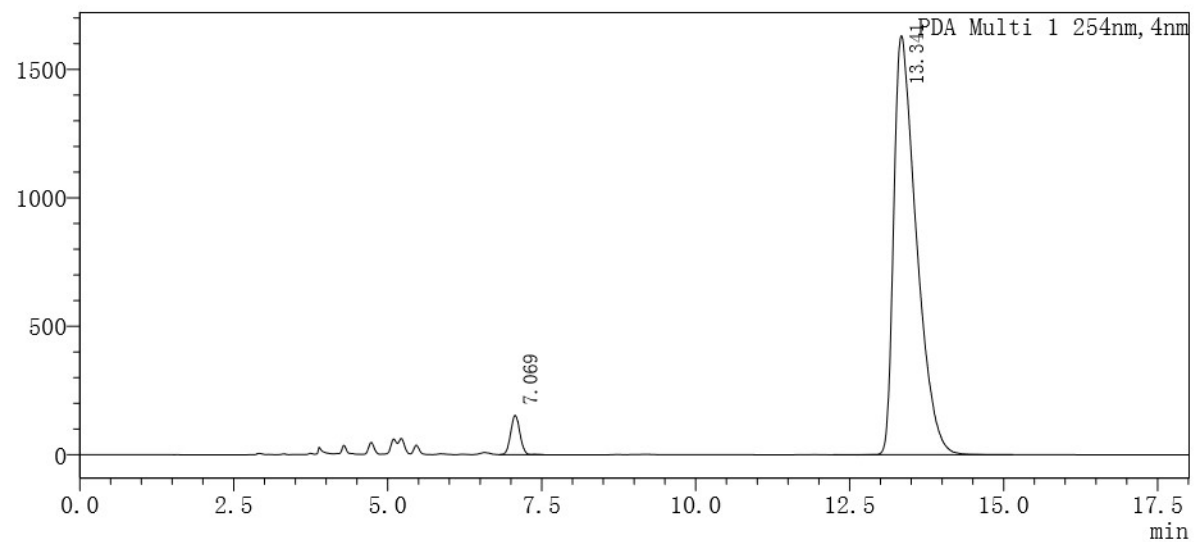

〈峰表〉

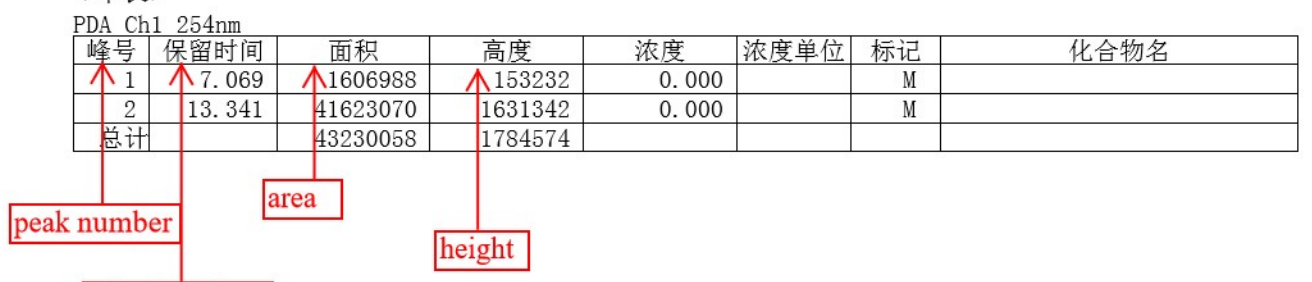

retention time 
(R, E)-3-(3-(3-bromophenyl)allyl)-3-methyl-2,3-dihydrobenzofuran (3ao)

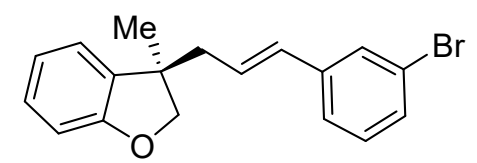

Chemical Formula: $\mathrm{C}_{18} \mathrm{H}_{17} \mathrm{BrO}$

Exact Mass: 328.0463

3ao was prepared according to general procedure using 1 a $(0.1 \mathrm{mmol}, 1.0$ equiv, 26.2 $\mathrm{mg})$ and $20(0.2 \mathrm{mmol}, 26 \mu \mathrm{L})$ and was purified by silica gel column chromatography (petroleum ether/EtOAc $=100 / 1 \sim 30 / 1)$ to obtain 3ao as colorless oil $(28.6 \mathrm{mg}, 87 \%$ yield). ${ }^{1} \mathrm{H}$ NMR $\left(400 \mathrm{MHz}, \mathrm{CDCl}_{3}\right): \delta 7.45(\mathrm{t}, J=1.8 \mathrm{~Hz}, 1 \mathrm{H}), 7.34$ (ddd, $J=7.7,1.9$, $1.3 \mathrm{~Hz}, 1 \mathrm{H}), 7.24-7.09(\mathrm{~m}, 4 \mathrm{H}), 6.91(\mathrm{td}, J=7.4,1.0 \mathrm{~Hz}, 1 \mathrm{H}), 6.82(\mathrm{ddd}, J=8.0,0.6$ $\mathrm{Hz}, 1 \mathrm{H}), 6.33(\mathrm{~d}, J=15.7 \mathrm{~Hz}, 1 \mathrm{H}), 6.11(\mathrm{dt}, J=15.7,7.5 \mathrm{~Hz}, 1 \mathrm{H}), 4.43(\mathrm{~d}, J=8.7 \mathrm{~Hz}$, 1H), $4.16(\mathrm{~d}, J=8.7 \mathrm{~Hz}, 1 \mathrm{H}), 2.51(\mathrm{dd}, J=7.5,1.3 \mathrm{~Hz}, 2 \mathrm{H}), 1.41(\mathrm{~s}, 3 \mathrm{H})$;

${ }^{13} \mathrm{C}$ NMR $\left(101 \mathrm{MHz}, \mathrm{CDCl}_{3}\right): \delta 159.5,139.4,134.6,13,130.1,130.0,128.9,128.3$, $127.4,124.8,122.9,122.7,120.5,109.8,81.9,45.6,44.2,24.9$;

HRMS: (APCI) calcd for $\mathrm{C}_{18} \mathrm{H}_{18} \mathrm{BrO}^{+}[\mathrm{M}+\mathrm{H}]^{+} 329.0536$; found 329.0528.

The enantiomeric purity was established by HPLC analysis using a chiral column: OD$\mathrm{H}$ column, $30^{\circ} \mathrm{C}, n$-Hexane/i-Propanol $=97 / 3$ as eluent, $254 \mathrm{~nm}, 1 \mathrm{~mL} / \mathrm{min} . \mathrm{tR}=5.2$ $\min$ (minor), $5.4 \min$ (major).

Optical Rotation: $[\alpha]_{\mathrm{D}}{ }^{30}-7.1\left(\mathrm{c} 0.4, \mathrm{CHCl}_{3}\right)$ for $93 \%$ ee.

Absolute stereochemistry was determined through analogy with 3ac. 

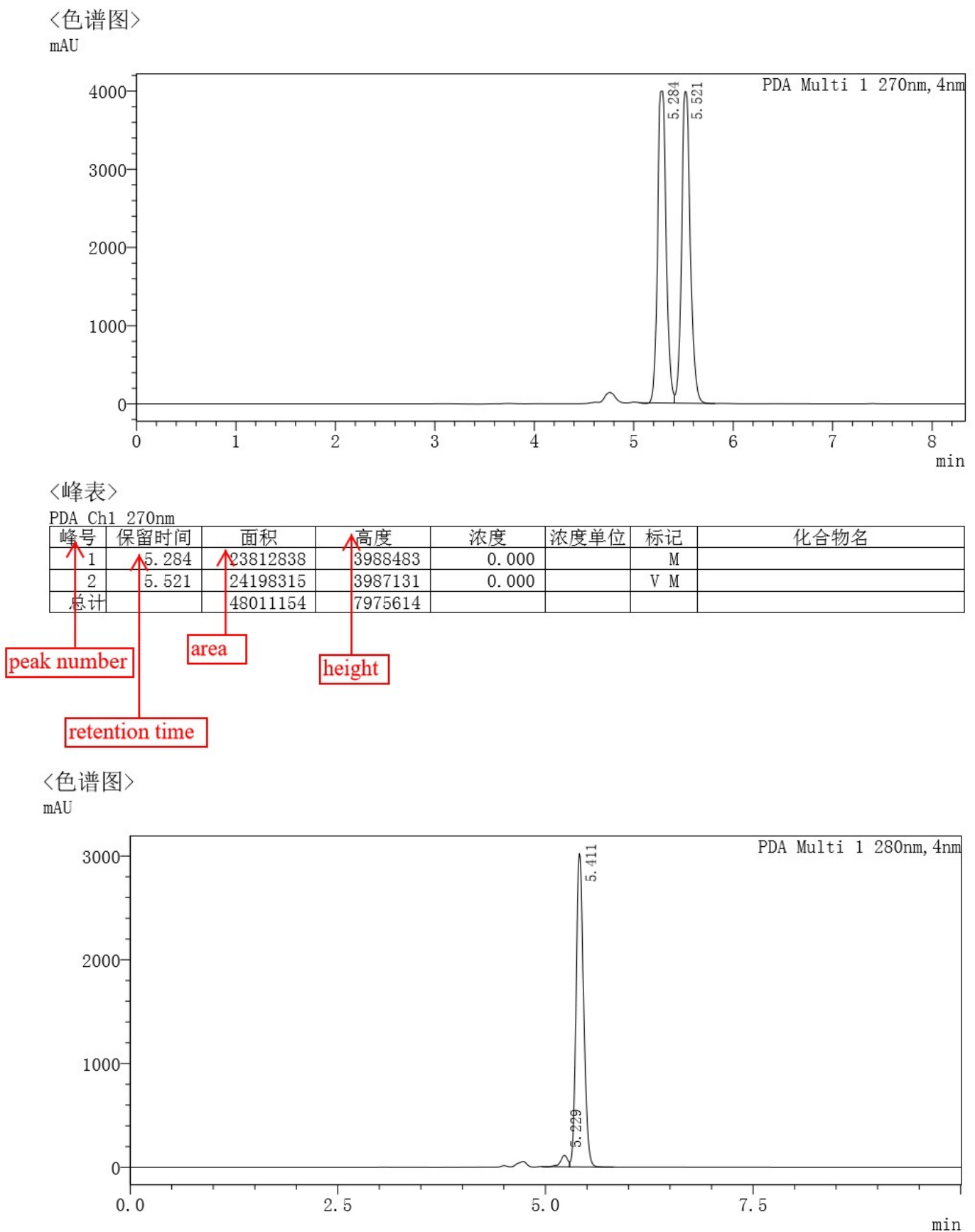

〈峰表〉

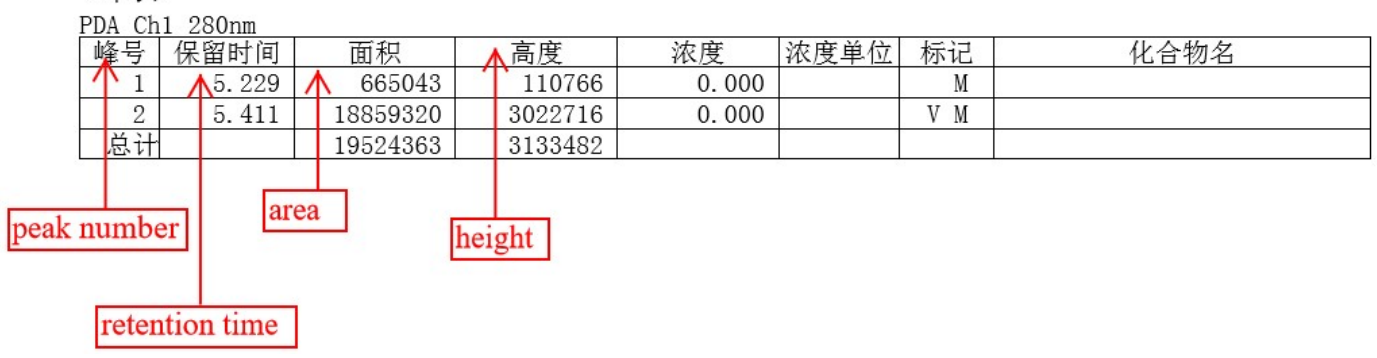


(R, E)-3-(3-(3,5-dimethoxyphenyl)allyl)-3-methyl-2,3-dihydrobenzofuran (3ap)<smiles>COc1cc(/C=C/C[C@]2(C)COc3ccccc32)cc(OC)c1</smiles>

Chemical Formula: $\mathrm{C}_{20} \mathrm{H}_{22} \mathrm{O}_{3}$

Exact Mass: 310.1569

3ap was prepared according to general procedure using 1 a $(0.1 \mathrm{mmol}, 1.0$ equiv, 26.2 $\mathrm{mg}$ ) and $2 \mathrm{p}(0.2 \mathrm{mmol}, 33 \mathrm{mg})$ and was purified by silica gel column chromatography (petroleum ether/EtOAc $=100 / 1 \sim 20 / 1)$ to obtain 3ap as colorless oil $(10.9 \mathrm{mg}, 35 \%$ yield). ${ }^{1} \mathrm{H}$ NMR (400 MHz, $\left.\mathrm{CDCl}_{3}\right): \delta 7.20-7.07(\mathrm{~m}, 2 \mathrm{H}), 6.89(\mathrm{td}, J=7.4,1.0 \mathrm{~Hz}, 1 \mathrm{H})$, $6.80(\mathrm{dt}, J=7.9,0.8 \mathrm{~Hz}, 1 \mathrm{H}), 6.47(\mathrm{~d}, J=2.2 \mathrm{~Hz}, 2 \mathrm{H}), 6.40-6.30(\mathrm{~m}, 2 \mathrm{H}), 6.16-6.02(\mathrm{~m}$, $1 \mathrm{H}), 4.43(\mathrm{~d}, J=8.6 \mathrm{~Hz}, 1 \mathrm{H}), 4.15(\mathrm{~d}, J=8.7 \mathrm{~Hz}, 1 \mathrm{H}), 3.79$ (s, 6H), 2.49 (dd, $J=7.5$, $1.2 \mathrm{~Hz}, 2 \mathrm{H}), 1.40(\mathrm{~s}, 3 \mathrm{H})$;

${ }^{13} \mathrm{C}$ NMR $\left(101 \mathrm{MHz}, \mathrm{CDCl}_{3}\right): \delta$ 160.9, 159.5, 139.4, 134.8, 133.4, 128.2, 126.3, 122.9, $120.5,109.7,104.3,99.3,82.0,55.3,45.6,44.2,24.9$;

HRMS: (ESI) calcd for $\mathrm{C}_{20} \mathrm{H}_{23} \mathrm{O}_{3}+[\mathrm{M}+\mathrm{H}]^{+} 311.1642$; found 311.1633 .

The enantiomeric purity was established by HPLC analysis using a chiral column: AD$\mathrm{H}$ column, $30{ }^{\circ} \mathrm{C}, n$-Hexane/i-Propanol $=85 / 15$ as eluent, $254 \mathrm{~nm}, 1 \mathrm{~mL} / \mathrm{min}$. $\mathrm{tR}=5.3$ $\min$ (major), $5.7 \min$ (minor).

Optical Rotation: $[\alpha]_{\mathrm{D}}{ }^{30}-22.3$ (c $\left.0.1, \mathrm{CHCl}_{3}\right)$ for $96 \%$ ee.

Absolute stereochemistry was determined through analogy with 3ac. 


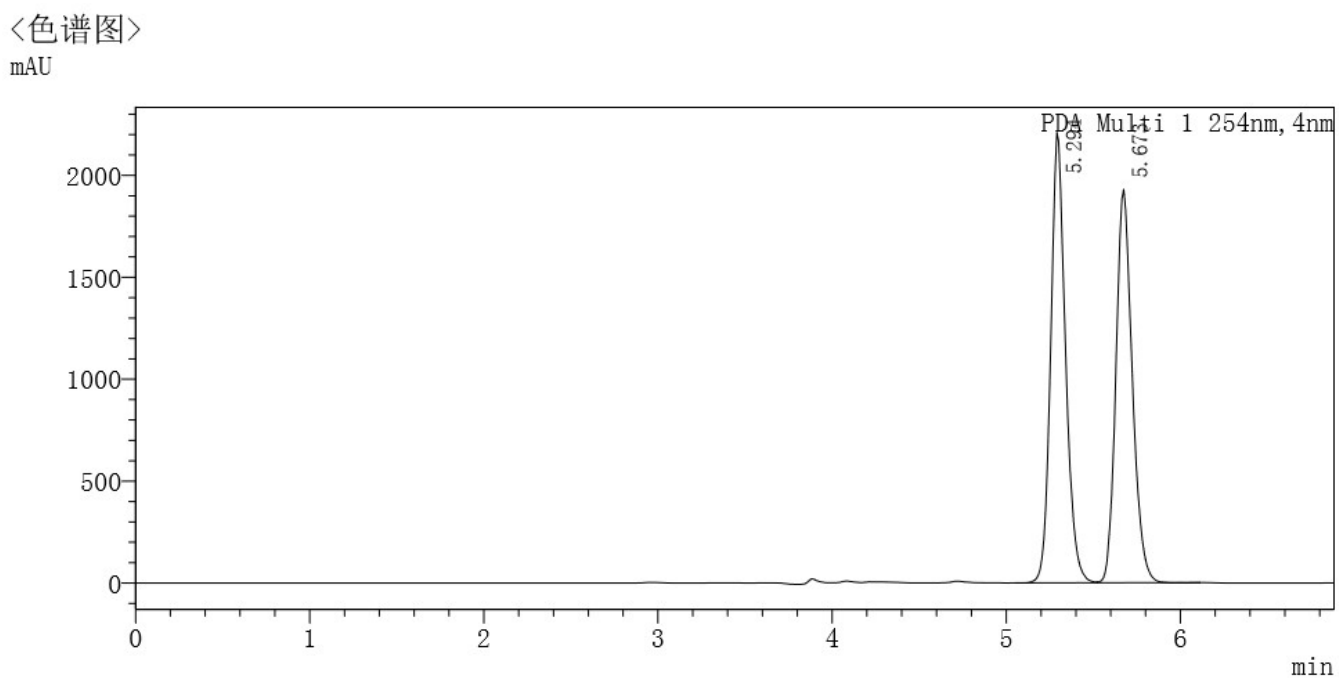

〈峰表〉

\begin{tabular}{|c|c|c|c|c|c|c|c|}
\hline | 峰号| & 保留时间 & 面积 & 高度 & 浓度 & 浓度单位 & 标记 & 化合物名 \\
\hline$\uparrow 1$ & \$5.294 & N13052027 & \$2210178 & 0.000 & & M & \\
\hline 2 & 5.673 & 12552447 & 1930180 & 0.000 & & V M & \\
\hline 总计 & & 25604474 & 4140358 & & & & \\
\hline numbe & & & height & & & & \\
\hline
\end{tabular}

retention time

〈色谱图〉

$\mathrm{mAU}$

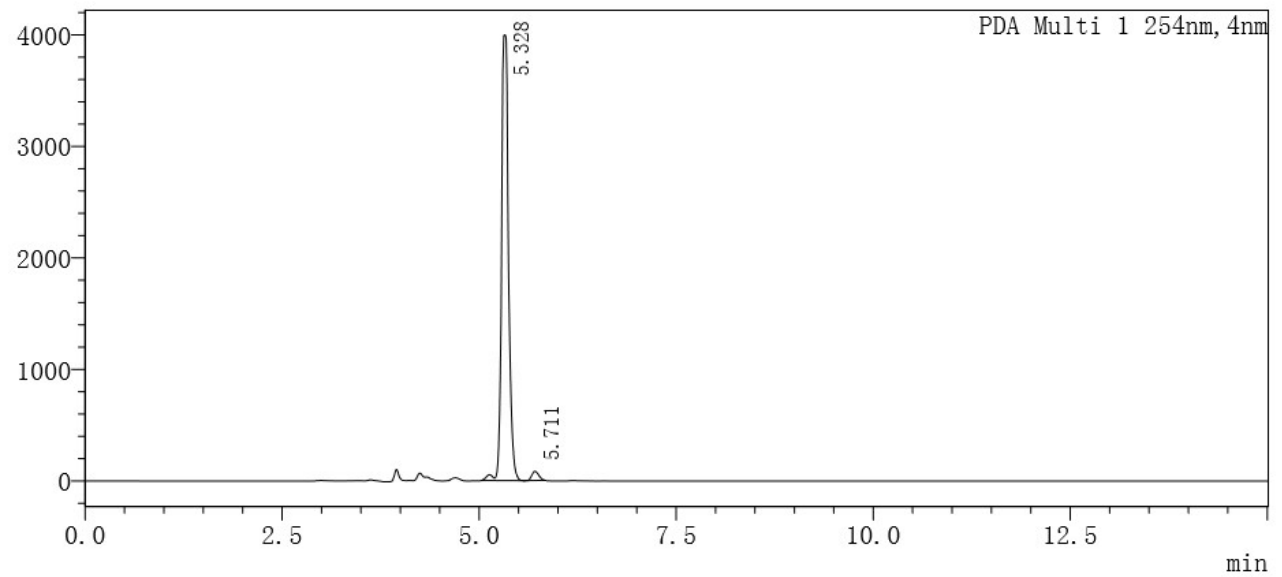

〈峰表〉

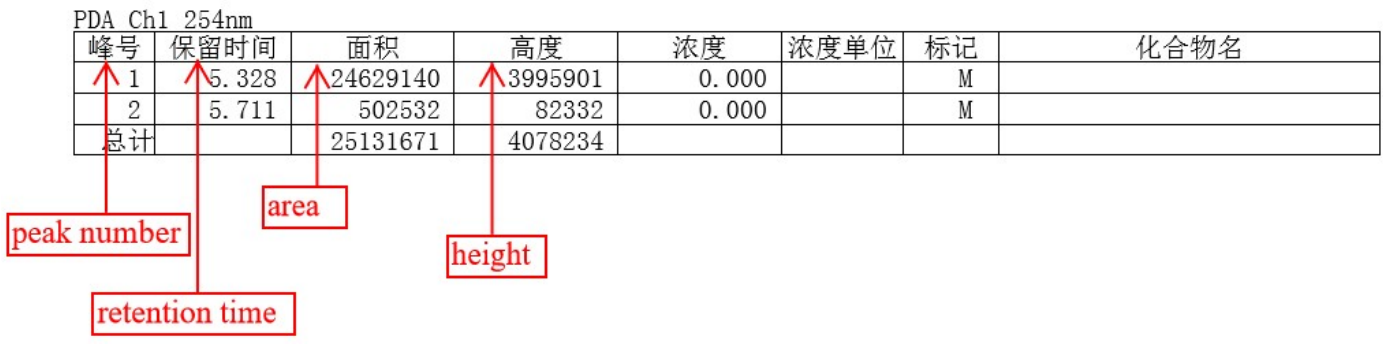


(R, E)-3-(3-(2-chlorophenyl)allyl)-3-methyl-2,3-dihydrobenzofuran (3aq)

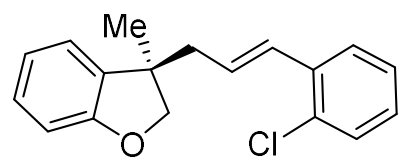

Chemical Formula: $\mathrm{C}_{18} \mathrm{H}_{17} \mathrm{ClO}$

Exact Mass: 284.0968

3aq was prepared according to general procedure using $1 \mathrm{a}(0.1 \mathrm{mmol}, 1.0$ equiv, 26.2 $\mathrm{mg})$ and $2 q(0.2 \mathrm{mmol}, 26 \mu \mathrm{L})$ and was purified by silica gel column chromatography (petroleum ether/EtOAc $=100 / 1 \sim 30 / 1)$ to obtain 3aq as colorless oil $(21.7 \mathrm{mg}, 76 \%$ yield). ${ }^{1} \mathrm{H}$ NMR $\left(400 \mathrm{MHz}, \mathrm{CDCl}_{3}\right): \delta 7.41(\mathrm{dd}, J=7.5,2.0 \mathrm{~Hz}, 1 \mathrm{H}), 7.33(\mathrm{dd}, J=7.7$, 1.6 Hz, 1H), 7.24-7.09 (m, 4H), $6.91(\mathrm{td}, J=7.4,1.0 \mathrm{~Hz}, 1 \mathrm{H}), 6.86-6.75(\mathrm{~m}, 2 \mathrm{H}), 6.06$ (dt, $J=15.4,7.5 \mathrm{~Hz}, 1 \mathrm{H}), 4.47(\mathrm{~d}, J=8.6 \mathrm{~Hz}, 1 \mathrm{H}), 4.18(\mathrm{~d}, J=8.7 \mathrm{~Hz}, 1 \mathrm{H}), 2.56(\mathrm{dd}, J$ $=7.5,1.4 \mathrm{~Hz}, 2 \mathrm{H}), 1.43(\mathrm{~s}, 3 \mathrm{H})$;

${ }^{13} \mathrm{C}$ NMR $\left(101 \mathrm{MHz}, \mathrm{CDCl}_{3}\right): \delta$ 159.5, 135.5, 134.6, 132.7, 129.8, 129.6, 128.8, 128.2, $126.9,126.8,122.9,120.6,109.7,81.9,45.6,44.4,25.1$;

HRMS: (ESI) calcd for $\mathrm{C}_{18} \mathrm{H}_{17} \mathrm{CINaO}^{+}[\mathrm{M}+\mathrm{Na}]^{+}$307.0860; found 307.0867.

The enantiomeric purity was established by HPLC analysis using a chiral column: OD$\mathrm{H}$ column, $30^{\circ} \mathrm{C}, n$-Hexane $/ \mathrm{i}$-Propanol $=98 / 2$ as eluent, $254 \mathrm{~nm}, 0.5 \mathrm{~mL} / \mathrm{min}$. tR $=11.3$ $\min$ (minor), 11.7 min (major).

Optical Rotation: $[\alpha]_{D}{ }^{30}-3.8$ (c $\left.0.3, \mathrm{CHCl}_{3}\right)$ for $92 \%$ ee.

Absolute stereochemistry was determined through analogy with 3ac. 
〈色谱图〉

mAU

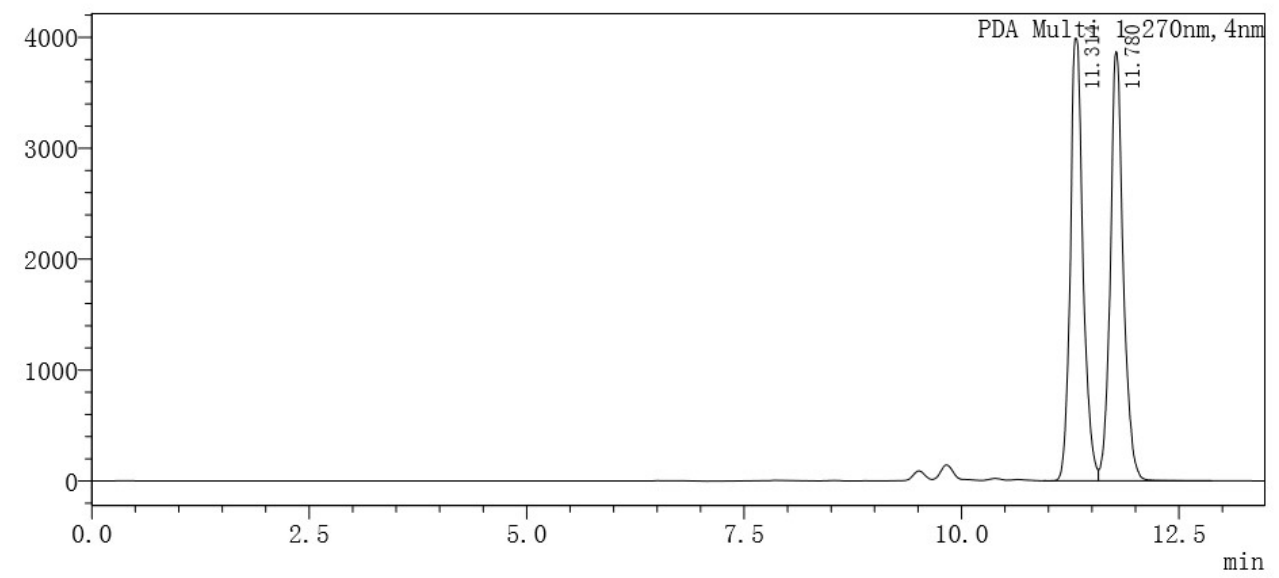

〈峰表〉

\begin{tabular}{|c|c|c|c|c|c|c|c|}
\hline 峰号 & 保留时间 & 面积 & 高度 & 浓度 & 浓度单位 & 标记 & 化合物名 \\
\hline 梠 & A1. 314 & $\$ 40675722$ & 3992904 & 0.000 & & M & \\
\hline 2 & 11.780 & 40029148 & 3871651 & 0.000 & & V M & \\
\hline 总计 & & 80704870 & 7864555 & & & & \\
\hline numb & & & height & & & & \\
\hline
\end{tabular}

retention time

〈色谱图〉

$\mathrm{mAU}$

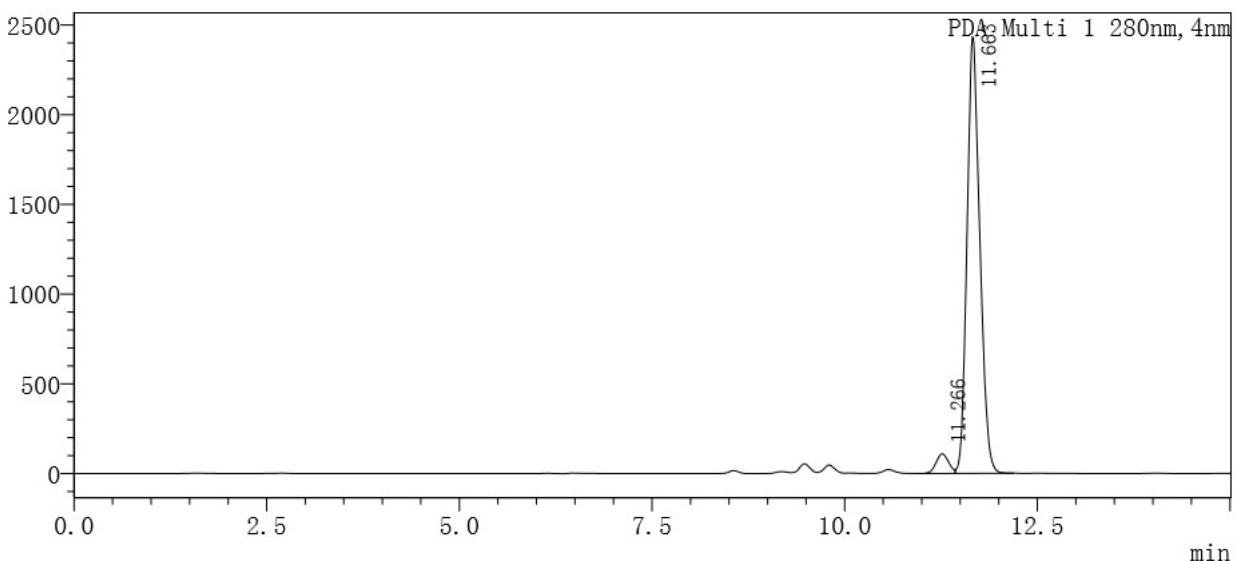

〈峰表〉

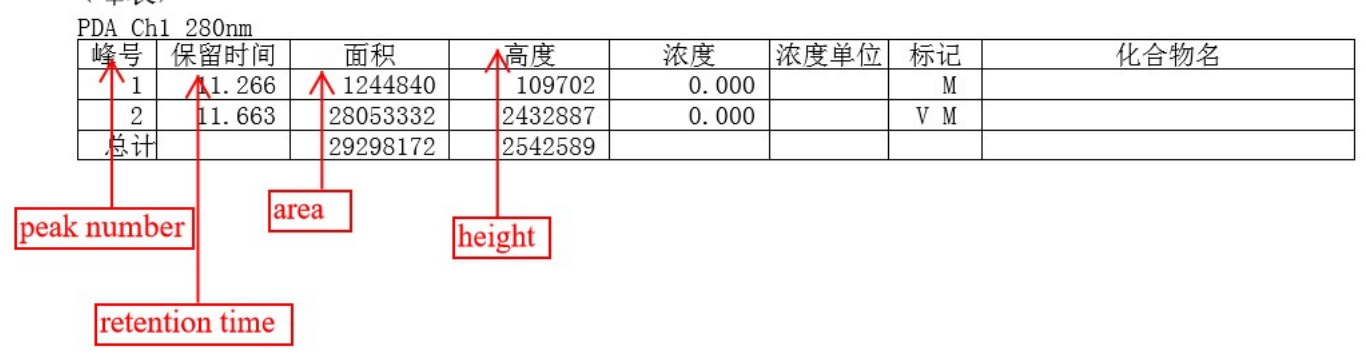


(R, E)-3-(3-(4-fluoro-3-methoxyphenyl)allyl)-3-methyl-2,3-dihydrobenzofuran (3ar)

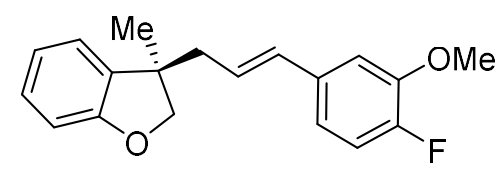

Chemical Formula: $\mathrm{C}_{19} \mathrm{H}_{19} \mathrm{FO}_{2}$

Exact Mass: 298.1369

3ar was prepared according to general procedure using $1 \mathrm{a}(0.1 \mathrm{mmol}, 1.0$ equiv, 26.2 $\mathrm{mg})$ and $2 \mathrm{r}(0.1 \mathrm{mmol}, 30.4 \mathrm{mg})$ and was purified by silica gel column chromatography (petroleum ether/EtOAc $=100 / 1 \sim 20 / 1)$ to obtain 3 ar as colorless oil $(16.1 \mathrm{mg}, 54 \%$ yield). ${ }^{1} \mathrm{H}$ NMR (400 MHz, $\left.\mathrm{CDCl}_{3}\right): \delta 7.19-7.09(\mathrm{~m}, 2 \mathrm{H}), 6.99(\mathrm{dd}, J=11.1,8.3 \mathrm{~Hz}, 1 \mathrm{H})$, 6.93-6.86 (m, 2H), 6.85-6.79 (m, 2H), $6.34(\mathrm{dd}, J=15.7,1.4 \mathrm{~Hz}, 1 \mathrm{H}), 5.99(\mathrm{dtd}, J=$ 15.7, 7.5, 0.6 Hz, 1H), $4.44(\mathrm{~d}, J=8.7 \mathrm{~Hz}, 1 \mathrm{H}), 4.16(\mathrm{~d}, J=8.7 \mathrm{~Hz}, 1 \mathrm{H}), 3.89(\mathrm{~s}, 3 \mathrm{H})$, 2.49 (dd, $J=7.5,1.3 \mathrm{~Hz}, 2 \mathrm{H}), 1.41(\mathrm{~s}, 3 \mathrm{H})$;

${ }^{13} \mathrm{C} \operatorname{NMR}\left(101 \mathrm{MHz}, \mathrm{CDCl}_{3}\right): \delta$ 159.5, $151.9(\mathrm{~d}, J=246.0 \mathrm{~Hz}), 147.6(\mathrm{~d}, J=11.0 \mathrm{~Hz})$, 134.7, 133.9, 132.5, 128.2, 125.6, 122.9, 120.5, 118.7 (d, $J=6.7 \mathrm{~Hz}), 116.0$ (d, $J=$ $18.8 \mathrm{~Hz}), 111.1,109.7,81.9,56.2,45.6,44.2,24.9$;

${ }^{19} \mathrm{~F} \mathrm{NMR}\left(376 \mathrm{MHz}, \mathrm{CDCl}_{3}\right): \delta-136.63 ;$

HRMS: (APCI) calcd for $\mathrm{C}_{19} \mathrm{H}_{20} \mathrm{FO}_{2}{ }^{+}[\mathrm{M}+\mathrm{H}]^{+} 299.1442$; found 299.1443.

The enantiomeric purity was established by HPLC analysis using a chiral column: OD$\mathrm{H}$ column, $30^{\circ} \mathrm{C}, n$-Hexane $/ i$-Propanol $=95 / 5$ as eluent, $254 \mathrm{~nm}, 1 \mathrm{~mL} / \mathrm{min} . \mathrm{tR}=5.5$ $\min ($ minor), $5.8 \min$ (major).

Optical Rotation: $[\alpha]_{D}^{28} 14.3$ (c $\left.0.3, \mathrm{CHCl}_{3}\right)$ for $93 \%$ ee.

Absolute stereochemistry was determined through analogy with 3ac. 
〈色谱图〉

$\mathrm{mAU}$

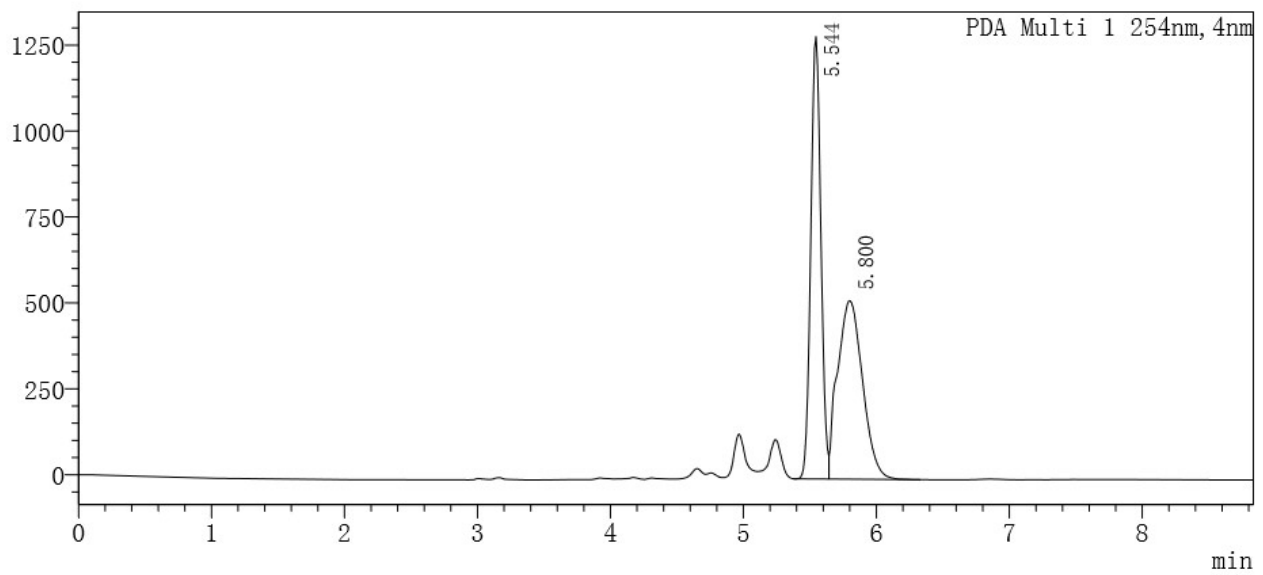

〈峰表〉

\begin{tabular}{|c|c|c|c|c|c|c|c|}
\hline 峰号 & 保留时间 & 面积 & 高度 & 浓度 & 浓度单位 & 标记 & 化合物名 \\
\hline 木 1 & \5. 544 & 16810429 & 1287663 & 0.000 & & $\mathrm{M}$ & \\
\hline 2 & 5.800 & 6777237 & 519817 & 0.000 & & V M & \\
\hline 总计 & & 13587666 & 1807480 & & & & \\
\hline
\end{tabular}

retention time

〈色谱图〉

maU

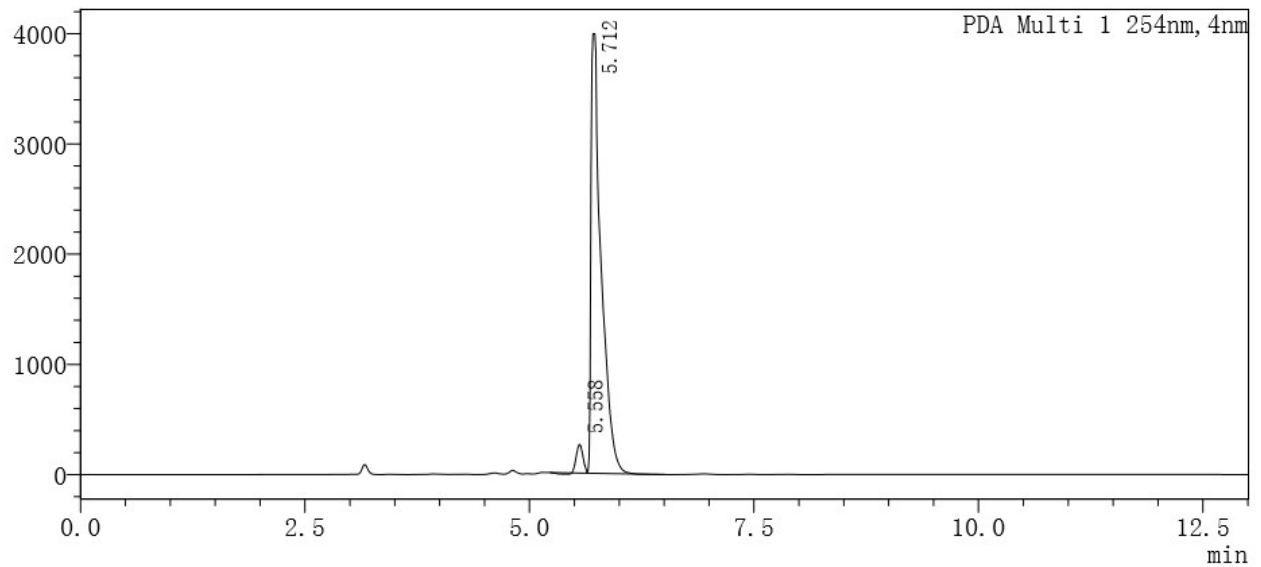

〈峰表〉

\begin{tabular}{|c|c|c|c|c|c|c|c|}
\hline 峰号 & 保留时间 & 面积 & 高度 & 浓度 & 浓度单位 & 标记 & 化合物名 \\
\hline 木 1 & 5.558 & \1212451 & 261455 & 0.000 & & $\mathrm{M}$ & \\
\hline 2 & 5.712 & 31305311 & 3987314 & 0.000 & & V M & \\
\hline 总计 & & 32517762 & 4248770 & & & & \\
\hline numb & & & ght & & & & \\
\hline
\end{tabular}

retention time 
(R, E)-3-(3-(3,4-dimethoxyphenyl)allyl)-3-methyl-2,3-dihydrobenzofuran (3as)

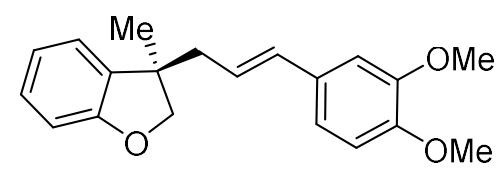

Chemical Formula: $\mathrm{C}_{20} \mathrm{H}_{22} \mathrm{O}_{3}$

Exact Mass: 310.1569

3as was prepared according to general procedure using $1 \mathrm{a}(0.1 \mathrm{mmol}, 1.0$ equiv, 26.2 $\mathrm{mg})$ and $2 \mathrm{~s}(0.1 \mathrm{mmol}, 32.8 \mathrm{mg})$ and was purified by silica gel column chromatography (petroleum ether/EtOAc $=100 / 1 \sim 20 / 1)$ to obtain 3as as colorless oil $(19.0 \mathrm{mg}, 61 \%$ yield). ${ }^{1} \mathrm{H}$ NMR $\left(400 \mathrm{MHz}, \mathrm{CDCl}_{3}\right): \delta 7.20-7.08(\mathrm{~m}, 2 \mathrm{H}), 6.98-6.76(\mathrm{~m}, 5 \mathrm{H}), 6.35(\mathrm{dt}, J$ $=15.5,1.3 \mathrm{~Hz}, 1 \mathrm{H}), 6.06-5.88(\mathrm{~m}, 1 \mathrm{H}), 4.45(\mathrm{~d}, J=8.6 \mathrm{~Hz}, 1 \mathrm{H}), 4.16(\mathrm{~d}, J=8.7 \mathrm{~Hz}$, 1H), 3.89 (s, 3H), 3.87 (s, 3H), 2.49 (dd, J = 7.5, $1.3 \mathrm{~Hz}, 2 \mathrm{H}), 1.40$ (s, 3H);

${ }^{13} \mathrm{C}$ NMR $\left(101 \mathrm{MHz}, \mathrm{CDCl}_{3}\right): \delta 159.5,148.9,148.5,134.9,133.1,130.5,128.1,123.7$, $122.9,120.4,119.0,111.1,109.6,108.8,81.9,55.9,55.8,45.7,44.2,24.9$;

HRMS: (ESI) calcd for $\mathrm{C}_{20} \mathrm{H}_{23} \mathrm{O}_{3}+[\mathrm{M}+\mathrm{H}]^{+} 311.1642$; found 311.1635 .

The enantiomeric purity was established by HPLC analysis using a chiral column: OD$\mathrm{H}$ column, $30{ }^{\circ} \mathrm{C}, n$-Hexane/i-Propanol $=90 / 10$ as eluent, $254 \mathrm{~nm}, 1 \mathrm{~mL} / \mathrm{min}$. $\mathrm{tR}=7.5$ $\min$ (minor), 9.1 min (major).

Optical Rotation: $[\alpha]_{D}^{28} 11.1$ (c $\left.0.3, \mathrm{CHCl}_{3}\right)$ for $91 \%$ ee. Absolute stereochemistry was determined through analogy with 3ac. 

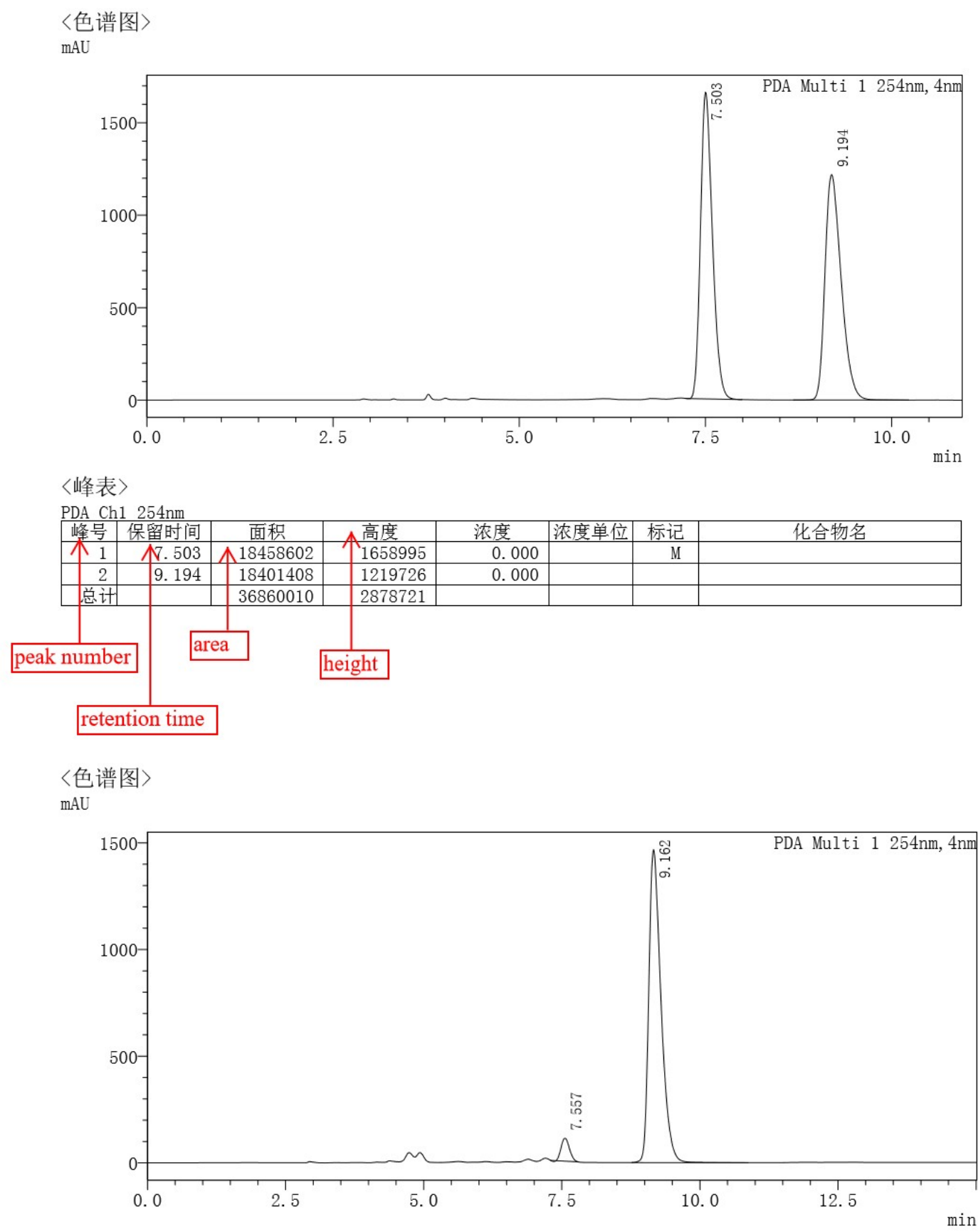

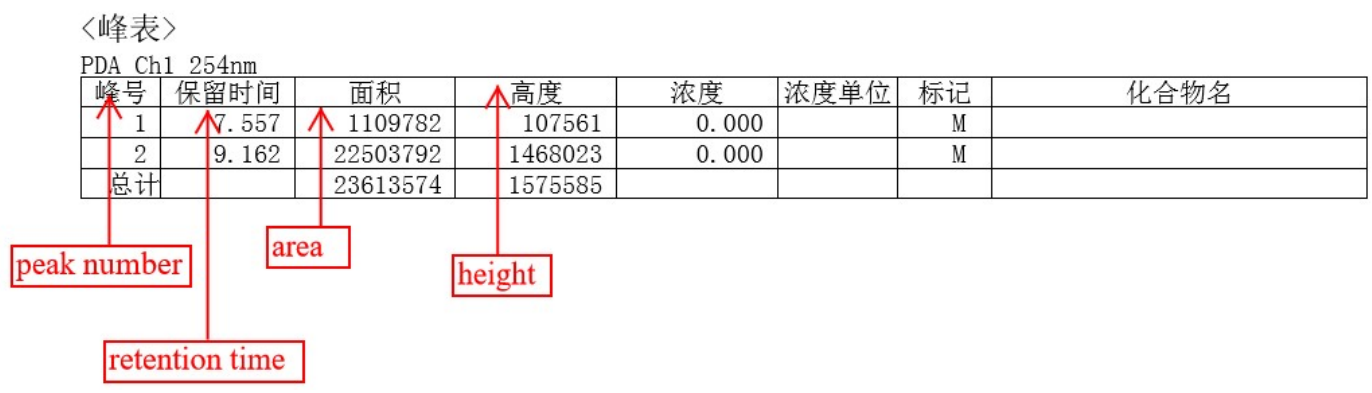


(R, E)-3-methyl-3-(3-(perfluorophenyl)allyl)-2,3-dihydrobenzofuran (3at)

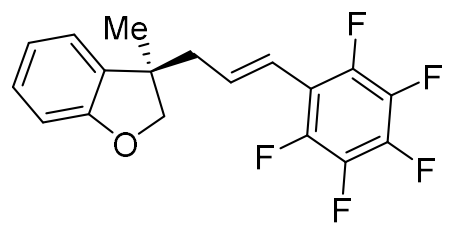

Chemical Formula: $\mathrm{C}_{18} \mathrm{H}_{13} \mathrm{~F}_{5} \mathrm{O}$

Exact Mass: 340.0887

3at was prepared according to general procedure using 1 a $(0.1 \mathrm{mmol}, 1.0$ equiv, 26.2 $\mathrm{mg})$ and $2 \mathrm{t}(0.5 \mathrm{mmol}, 69 \mu \mathrm{L})$ and was purified by silica gel column chromatography (petroleum ether/EtOAc $=100 / 1 \sim 30 / 1)$ to obtain 3at as colorless oil $(21.1 \mathrm{mg}, 62 \%$ yield). ${ }^{1} \mathrm{H}$ NMR (400 MHz, $\left.\mathrm{CDCl}_{3}\right): \delta 7.24-7.07(\mathrm{~m}, 2 \mathrm{H}), 6.90(\mathrm{td}, J=7.5,1.0 \mathrm{~Hz}, 1 \mathrm{H})$, $6.81(\mathrm{dt}, J=8.0,0.8 \mathrm{~Hz}, 1 \mathrm{H}), 6.42(\mathrm{dt}, J=16.2,7.4 \mathrm{~Hz}, 1 \mathrm{H}), 6.28(\mathrm{dt}, J=16.3,1.3 \mathrm{~Hz}$, 1H), $4.42(\mathrm{~d}, J=8.7 \mathrm{~Hz}, 1 \mathrm{H}), 4.17(\mathrm{~d}, J=8.8 \mathrm{~Hz}, 1 \mathrm{H}), 2.73-2.43(\mathrm{~m}, 2 \mathrm{H}), 1.42(\mathrm{~s}, 3 \mathrm{H})$; ${ }^{13} \mathrm{C} \mathrm{NMR}\left(101 \mathrm{MHz}, \mathrm{CDCl}_{3}\right): \delta 159.5,136.0,134.1,128.4,122.8,120.6,117.4,109.8$, $81.9,45.6,45.5,24.7$

${ }^{19} \mathrm{~F} \mathrm{NMR}\left(376 \mathrm{MHz}, \mathrm{CDCl}_{3}\right): \delta-143.40(\mathrm{~m}),-156.90(\mathrm{t}, J=20.9 \mathrm{~Hz}),-163.02(\mathrm{~m})$;

HRMS: (APCI) calcd for $\mathrm{C}_{18} \mathrm{H}_{14} \mathrm{~F}_{5} \mathrm{O}^{+}[\mathrm{M}+\mathrm{H}]^{+} 341.0959$; found 341.0958 .

The enantiomeric purity was established by HPLC analysis using a chiral column: OJ$\mathrm{H}$ column, $30{ }^{\circ} \mathrm{C}, n$-Hexane/i-Propanol $=97 / 3$ as eluent, $254 \mathrm{~nm}, 1 \mathrm{~mL} / \mathrm{min} . \mathrm{tR}=4.7$ $\min$ (minor), $4.9 \mathrm{~min}$ (major).

Optical Rotation: $[\alpha]_{D}^{30}-14.2$ (c $\left.0.4, \mathrm{CHCl}_{3}\right)$ for $90 \%$ ee.

Absolute stereochemistry was determined through analogy with 3ac. 
〈色谱图〉

$\mathrm{mAU}$

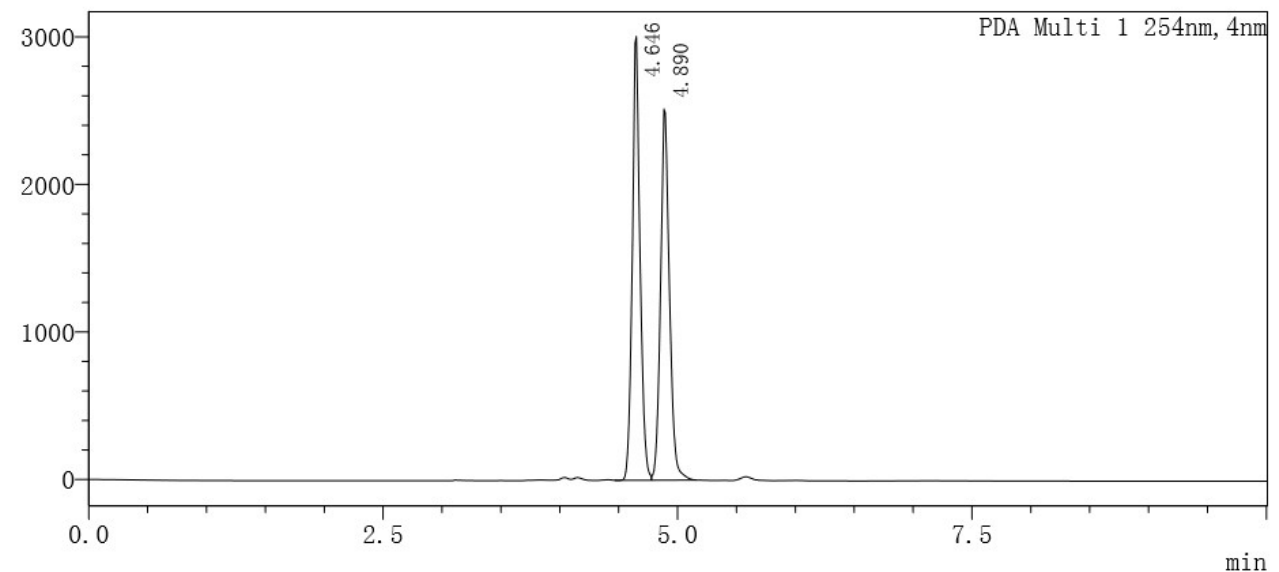

〈峰表〉

\begin{tabular}{|c|c|c|c|c|c|c|c|}
\hline 峰号 & 保留时间 & 面积 & 高度 & 浓度 & 浓度单位 & 标记 & 化合物名 \\
\hline $\begin{array}{ll} \\
\end{array}$ & 森.646 & \$13651633 & 3009043 & 0.000 & & M & \\
\hline 2 & 4.890 & 13161968 & 2516941 & 0.000 & & V M & \\
\hline 总计 & & 26813601 & 5525984 & & & & \\
\hline numbe & & & height & & & & \\
\hline
\end{tabular}

retention time

〈色谱图〉

$\mathrm{mAU}$

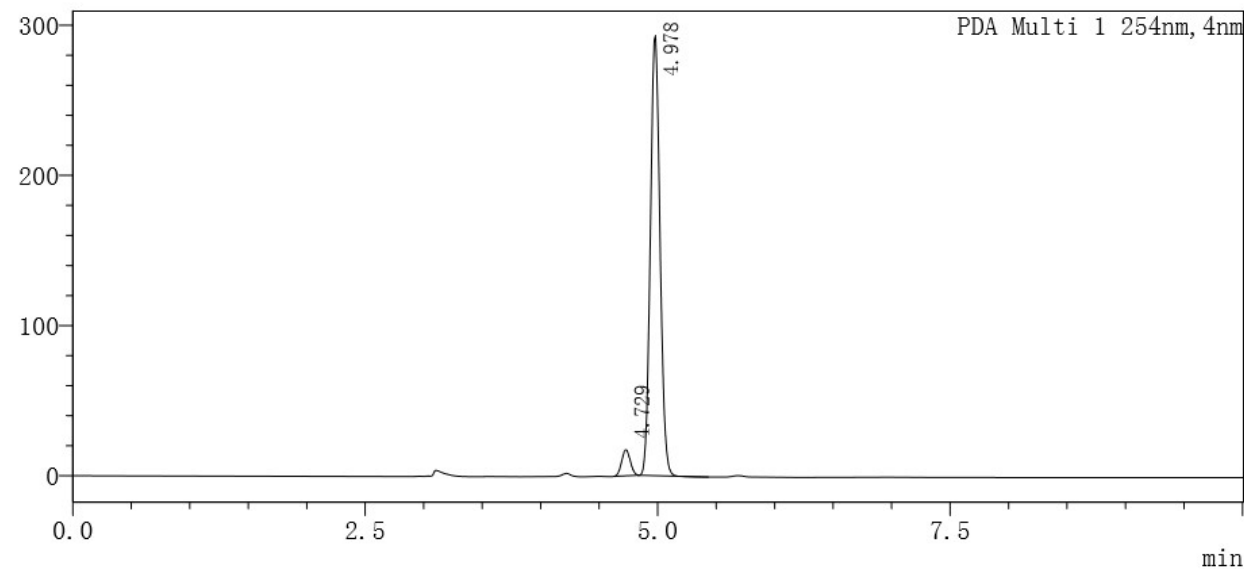

〈峰表〉

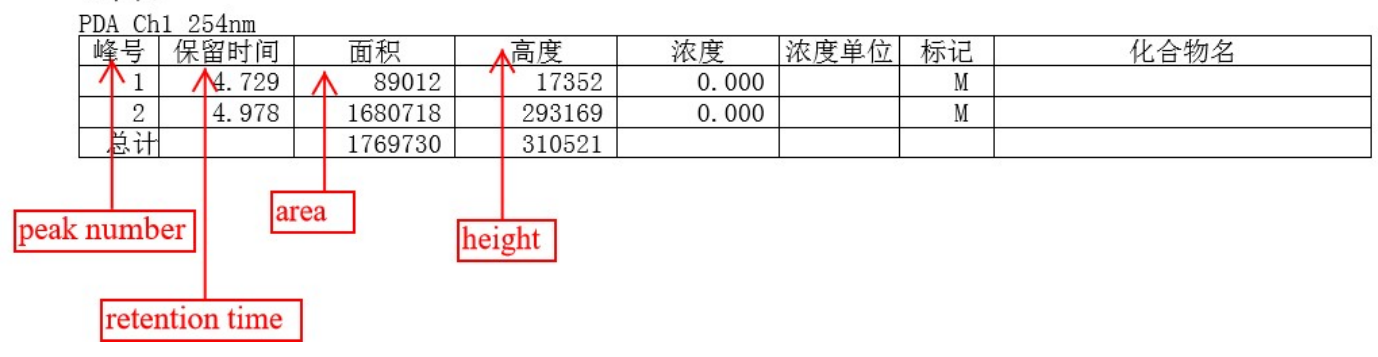


(R, E)-3-(3-(4-bromophenyl)allyl)-5-(tert-butyl)-3-methyl-2,3-dihydrobenzofuran (3bk)

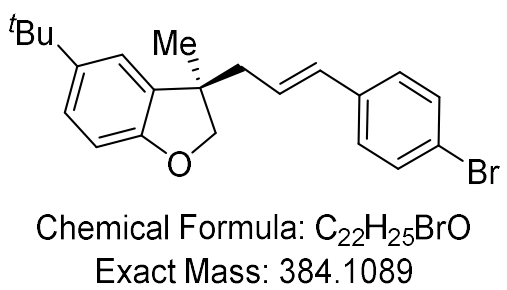

3bk was prepared according to general procedure using $\mathbf{1 b}(0.1 \mathrm{mmol}, 1.0$ equiv, 31.8 $\mathrm{mg})$ and $2 \mathrm{k}(0.2 \mathrm{mmol}, 26.2 \mu \mathrm{L})$ and was purified by silica gel column chromatography (petroleum ether/EtOAc $=100 / 1 \sim 30 / 1)$ to obtain $3 \mathbf{b k}$ as colorless oil $(18.4 \mathrm{mg}, 48 \%$ yield). ${ }^{1} \mathrm{H}$ NMR $\left(400 \mathrm{MHz}, \mathrm{CDCl}_{3}\right): \delta 7.50-7.38(\mathrm{~m}, 2 \mathrm{H}), 7.17(\mathrm{dd}, J=8.5, \mathrm{~Hz}, 3 \mathrm{H})$, $7.11(\mathrm{~d}, J=\mathrm{Hz}, 1 \mathrm{H}), 6.74(\mathrm{~d}, J=8.4 \mathrm{~Hz}, 1 \mathrm{H}), 6.38-6.27(\mathrm{~m}, 1 \mathrm{H}), 6.12(\mathrm{dt}, J=15.8$, $7.5 \mathrm{~Hz}, 1 \mathrm{H}), 4.41$ (d, J = 8.6 Hz, 1H), 4.15 (d, J = 8.6 Hz, 1H), 2.49 (dd, J= 7.5, $1.4 \mathrm{~Hz}$, $2 \mathrm{H}), 1.41(\mathrm{~s}, 3 \mathrm{H}), 1.30(\mathrm{~s}, 9 \mathrm{H})$;

${ }^{13} \mathrm{C}$ NMR $\left(101 \mathrm{MHz}, \mathrm{CDCl}_{3}\right): \delta 157.2,143.5,136.3,134.1,132.2,131.6,127.6,126.7$, $124.9,120.9,119.8,108.8,82.3,45.8,44.3,34.4,31.7,24.6$;

HRMS: (APCI) calcd for $\mathrm{C}_{22} \mathrm{H}_{26} \mathrm{BrO}^{+}[\mathrm{M}+\mathrm{H}]^{+} 385.1162$; found 385.1165.

The enantiomeric purity was established by HPLC analysis using a chiral column: OD$\mathrm{H}$ column, $30{ }^{\circ} \mathrm{C}, n$-Hexane/ $\mathrm{i}$-Propanol $=98 / 2$ as eluent, $254 \mathrm{~nm}, 0.5 \mathrm{~mL} / \mathrm{min} . \mathrm{tR}=8.5$ $\min$ (minor), 8.9 min (major).

Optical Rotation: $[\alpha]_{\mathrm{D}}{ }^{30} 30.1$ (c $0.5, \mathrm{CHCl}_{3}$ ) for $92 \%$ ee.

Absolute stereochemistry was determined through analogy with 3ac. 
〈色谱图〉

$\mathrm{mAU}$
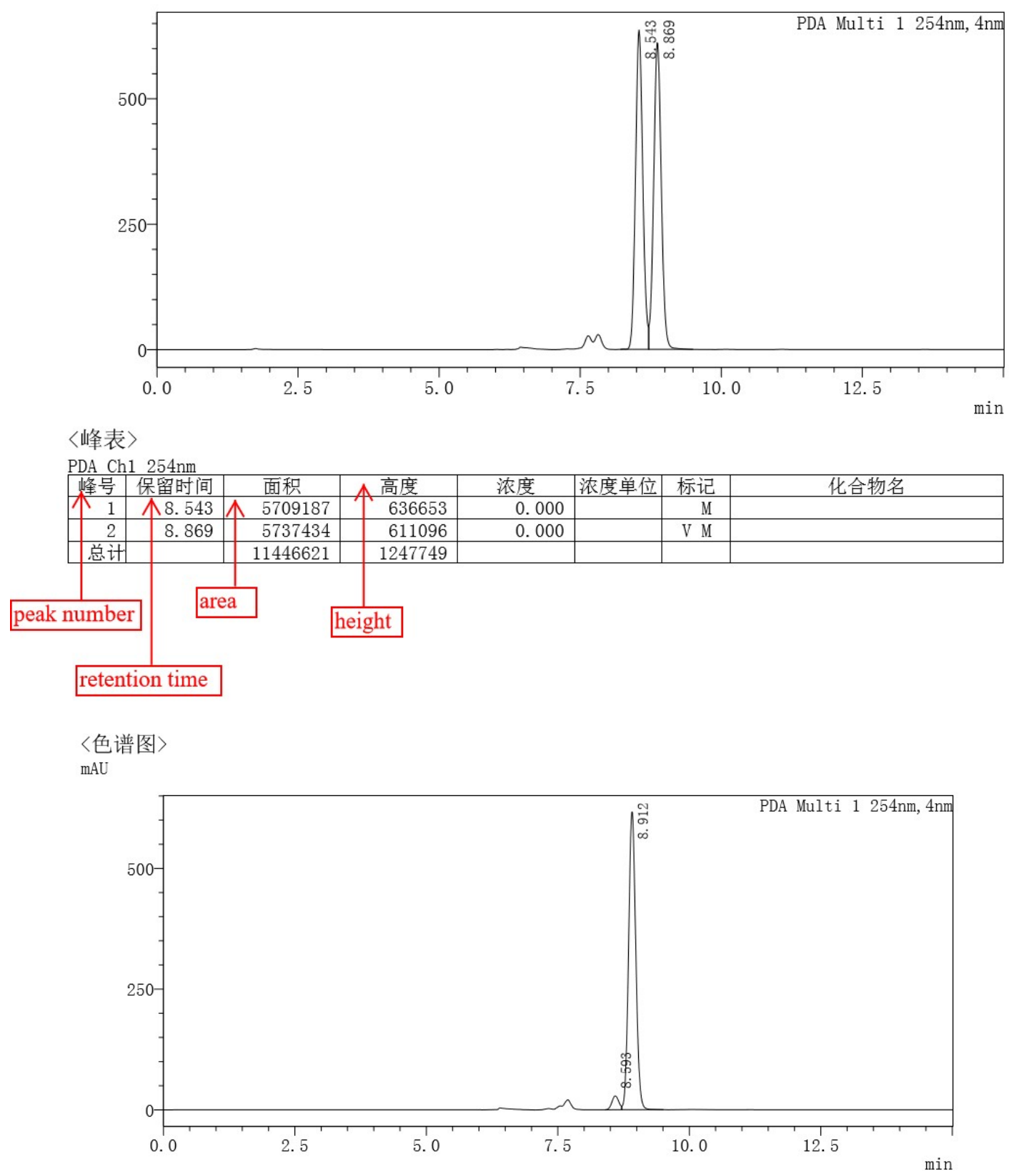

〈峰表〉

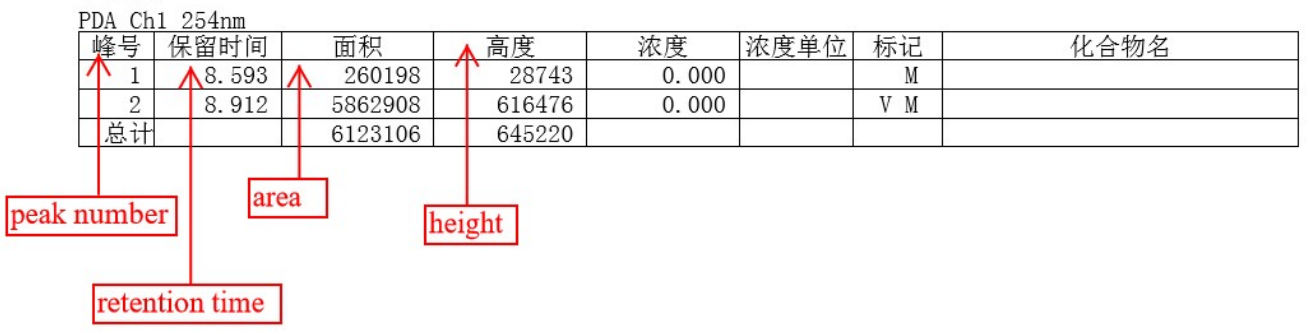


(R, E)-3-(3-(4-bromophenyl)allyl)-3-methyl-5-phenyl-2,3-dihydrobenzofuran (3ck)

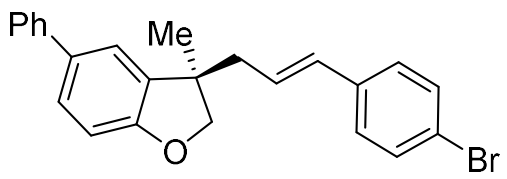

Chemical Formula: $\mathrm{C}_{24} \mathrm{H}_{21} \mathrm{BrO}$

Exact Mass: 404.0776

3ck was prepared according to general procedure using $1 \mathrm{c}(0.1 \mathrm{mmol}, 1.0$ equiv, 33.8 $\mathrm{mg})$ and $2 \mathbf{k}(0.2 \mathrm{mmol}, 26.2 \mu \mathrm{L})$ and was purified by silica gel column chromatography (petroleum ether/EtOAc $=100 / 1 \sim 30 / 1)$ to obtain 3ck as colorless oil $(22.7 \mathrm{mg}, 56 \%$ yield). ${ }^{1} \mathrm{H}$ NMR (400 MHz, $\left.\mathrm{CDCl}_{3}\right): \delta$ 7.59-7.51 (m, 2H), 7.48-7.37 (m, 5H), 7.36-7.28 (m, 2H), 7.22-7.15 (m, 2H), $6.88(\mathrm{~d}, J=8.3 \mathrm{~Hz}, 1 \mathrm{H}), 6.60-6.31(\mathrm{~m}, 1 \mathrm{H}), 6.13(\mathrm{dt}, J=$ 15.7, 7.5 Hz, 1H), 4.50 (d, $J=8.7 \mathrm{~Hz}, 1 \mathrm{H}), 4.22$ (d, $J=8.7 \mathrm{~Hz}, 1 \mathrm{H}$ ), 2.55 (ddd, $J=7.7$, 3.1, $1.3 \mathrm{~Hz}, 2 \mathrm{H}), 1.46(\mathrm{~s}, 3 \mathrm{H})$;

${ }^{13} \mathrm{C} \mathrm{NMR}\left(101 \mathrm{MHz}, \mathrm{CDCl}_{3}\right): \delta$ 159.2, 141.3, 136.2, 135.3, 134.1, 132.5, 131.6, 128.7, $127.7,127.4,126.8,126.6,126.4,121.8,121.0,109.9,82.3,45.7,44.3,25.0$;

HRMS: (APCI) calcd for $\mathrm{C}_{24} \mathrm{H}_{22} \mathrm{BrO}^{+}[\mathrm{M}+\mathrm{H}]^{+} 405.0849$; found 405.0848.

The enantiomeric purity was established by HPLC analysis using a chiral column: OD$\mathrm{H}$ column, $30{ }^{\circ} \mathrm{C}, n$-Hexane/i-Propanol $=98 / 2$ as eluent, $254 \mathrm{~nm}, 1 \mathrm{~mL} / \mathrm{min}$. $\mathrm{tR}=8.4$ $\min$ (minor), $9.5 \min$ (major).

Optical Rotation: $[\alpha]_{D}^{28} 153.1$ (c $0.5, \mathrm{CHCl}_{3}$ ) for $91 \%$ ee.

Absolute stereochemistry was determined through analogy with 3ac. 
〈色谱图〉

mAU

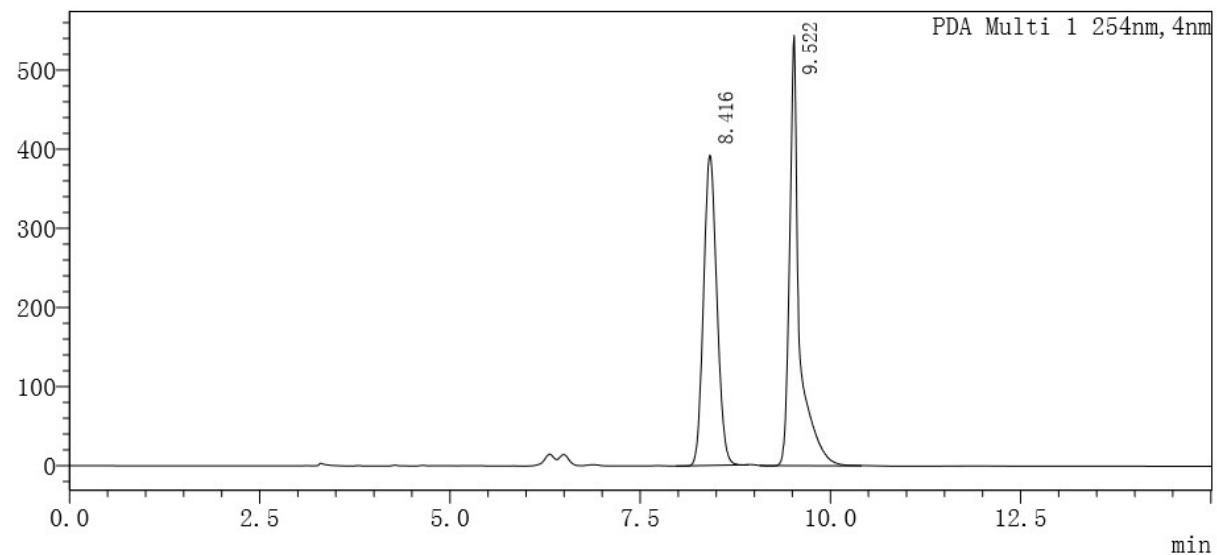

〈峰表〉

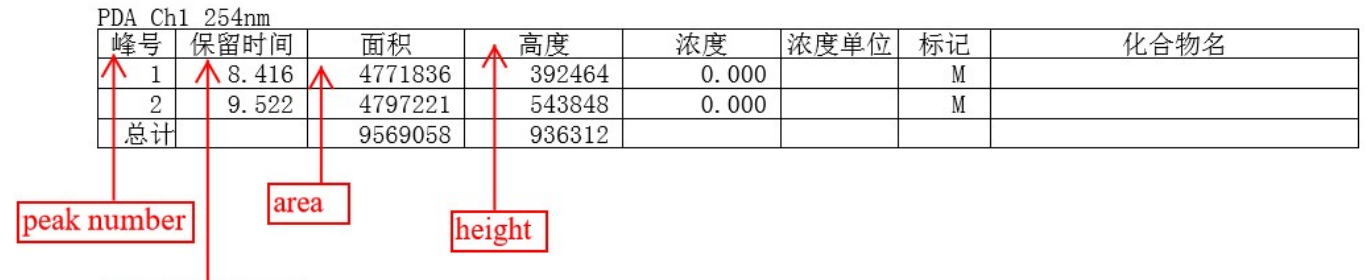

retention time

〈色谱图〉

$\mathrm{mAU}$

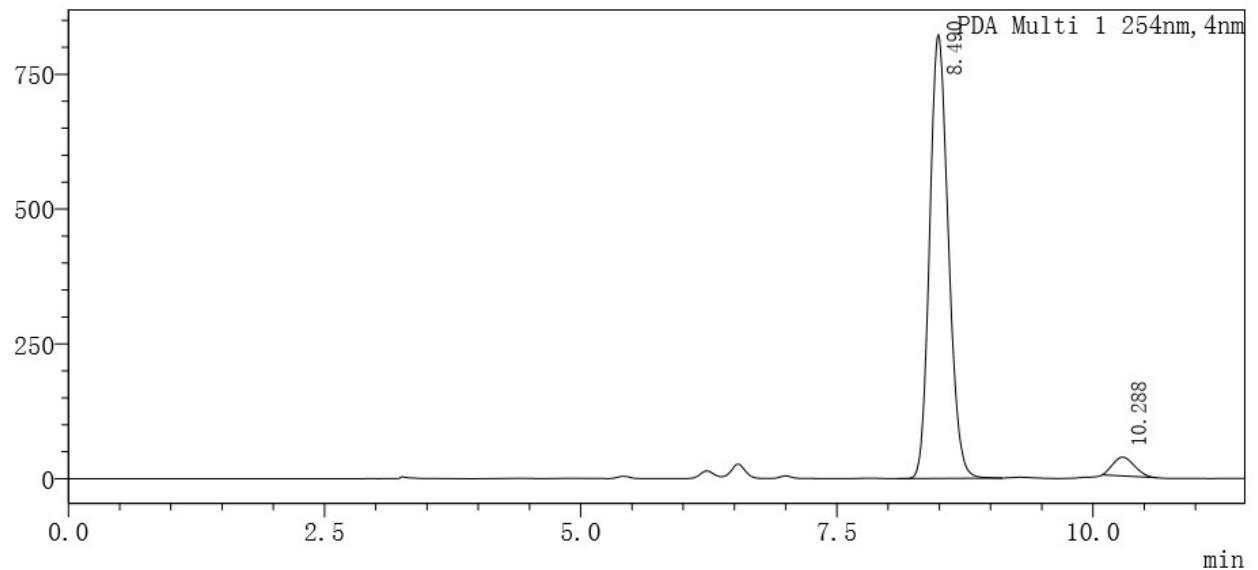

〈峰表〉

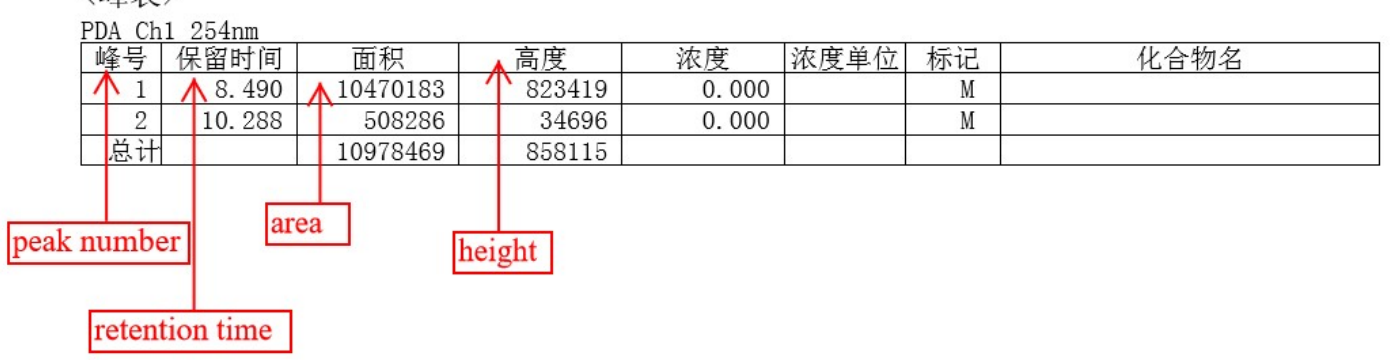


(R, E)-3-(3-(4-bromophenyl)allyl)-5-chloro-3-methyl-2,3-dihydrobenzofuran (3dk)

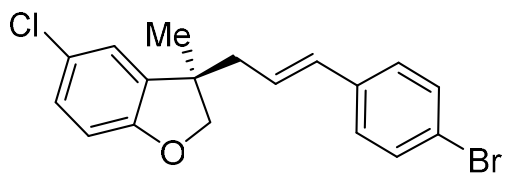

Chemical Formula: $\mathrm{C}_{18} \mathrm{H}_{16} \mathrm{BrClO}$

Exact Mass: 362.0073

3dk was prepared according to general procedure using $\mathbf{1 d}(0.1 \mathrm{mmol}, 1.0$ equiv, 29.6 $\mathrm{mg})$ and $2 \mathbf{k}(0.2 \mathrm{mmol}, 26.2 \mu \mathrm{L})$ and was purified by silica gel column chromatography (petroleum ether/EtOAc $=100 / 1 \sim 30 / 1)$ to obtain $3 \mathbf{d k}$ as colorless oil $(19.3 \mathrm{mg}, 53 \%$ yield). ${ }^{1} \mathrm{H}$ NMR (400 MHz, $\left.\mathrm{CDCl}_{3}\right): \delta$ 7.54-7.34 (m, 2H), 7.19-7.12 (m, 2H), 7.08 (dd, J $=8.4,2.3 \mathrm{~Hz}, 1 \mathrm{H}), 7.04(\mathrm{~d}, J=2.0 \mathrm{~Hz}, 1 \mathrm{H}), 6.70(\mathrm{~d}, J=8.5 \mathrm{~Hz}, 1 \mathrm{H}), 6.44-6.25(\mathrm{~m}, 1 \mathrm{H})$, $6.03(\mathrm{dt}, J=15.7,7.5 \mathrm{~Hz}, 1 \mathrm{H}), 4.43(\mathrm{~d}, J=8.7 \mathrm{~Hz}, 1 \mathrm{H}), 4.16(\mathrm{~d}, J=8.7 \mathrm{~Hz}, 1 \mathrm{H}), 2.46$ (ddd, $J=7.7,2.3,1.3 \mathrm{~Hz}, 2 \mathrm{H}), 1.38(\mathrm{~s}, 3 \mathrm{H})$;

${ }^{13} \mathrm{C}$ NMR $\left(101 \mathrm{MHz}, \mathrm{CDCl}_{3}\right): \delta$ 158.2, 136.6, 136.0, 132.7, 131.6, 128.2, 127.7, 125.9, $125.2,123.1,121.1,110.7,82.3,45.9,44.1,25.0$;

HRMS: (APCl) calcd for $\mathrm{C}_{18} \mathrm{H}_{17} \mathrm{BrClO}^{+}[\mathrm{M}+\mathrm{H}]^{+}$363.0146; found 363.0158.

The enantiomeric purity was established by HPLC analysis using a chiral column: OD$\mathrm{H}$ column, $30^{\circ} \mathrm{C}, n$-Hexane $/ \mathrm{i}$-Propanol $=98 / 2$ as eluent, $254 \mathrm{~nm}, 0.5 \mathrm{~mL} / \mathrm{min}$. tR $=11.2$ $\min$ (minor), 12.4 min (major).

Optical Rotation: $[\alpha]_{\mathrm{D}}{ }^{30} 71.4\left(\mathrm{c} 0.5, \mathrm{CHCl}_{3}\right)$ for $90 \%$ ee.

Absolute stereochemistry was determined through analogy with 3ac. 
〈色谱图〉

$\mathrm{mAU}$

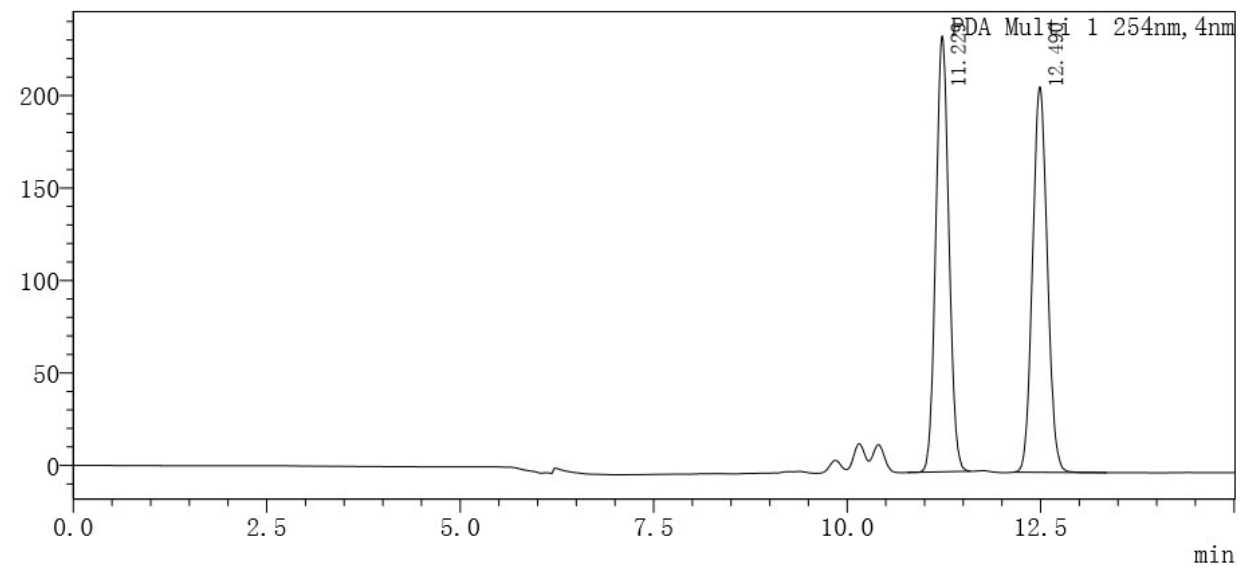

〈峰表〉

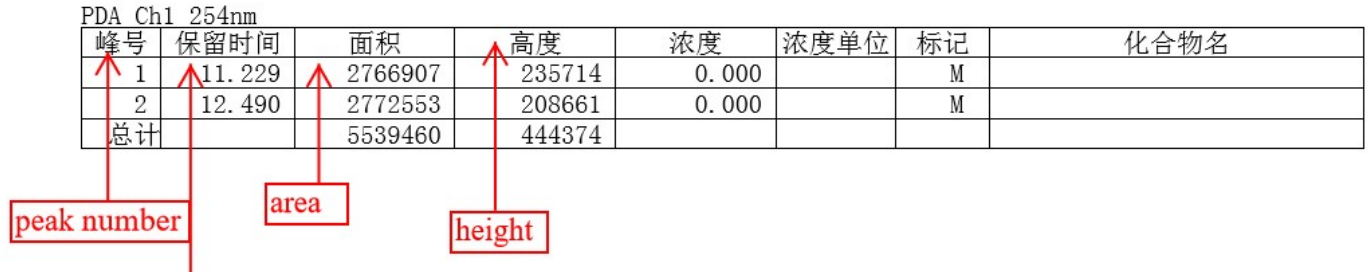

retention time

〈色谱图〉

mAU

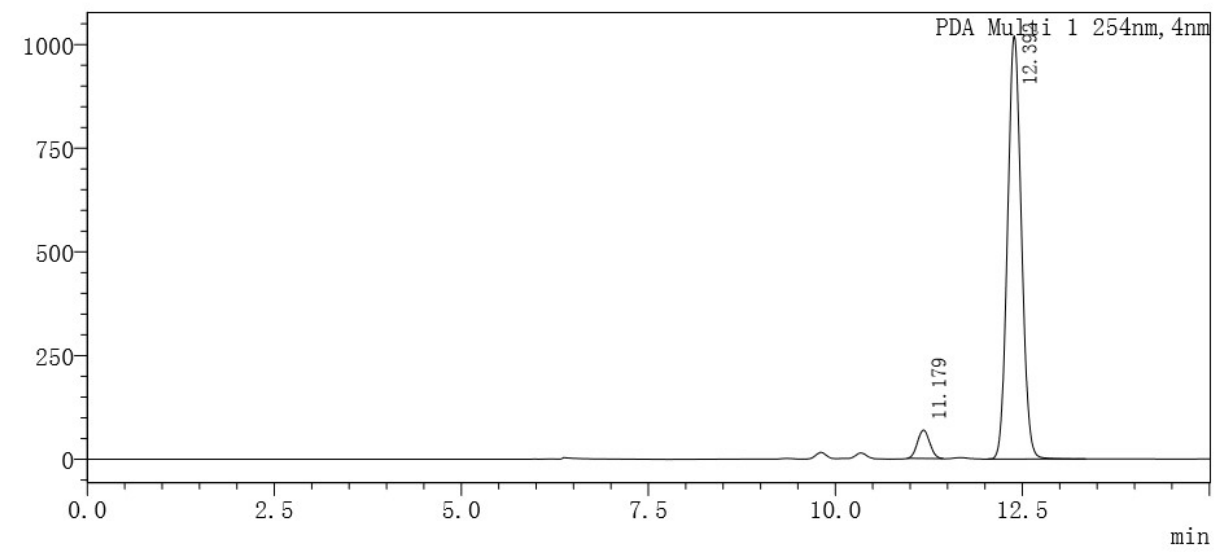

〈峰表〉

PDA Ch1 254nm

峰号 保留时间

\begin{tabular}{|c|c|c|c|c|c|c|c|}
\hline 峰号 & 保留时间 & 面积 & 高度 & 浓度 & 浓度单位 & 标记 & 化合物名 \\
\hline 本 1 & $\$ 11.179$ & 779201 & 68666 & 0.000 & & M & \\
\hline 2 & 12. 392 & 13133568 & 1020302 & 0.000 & & M & \\
\hline 总计 & & 13912769 & 1088968 & & & & \\
\hline number & $\operatorname{ar}$ & & height & & & & \\
\hline
\end{tabular}

retention time 
(R, E)-5-bromo-3-(3-(4-bromophenyl)allyl)-3-methyl-2,3-dihydrobenzofuran (3ek)

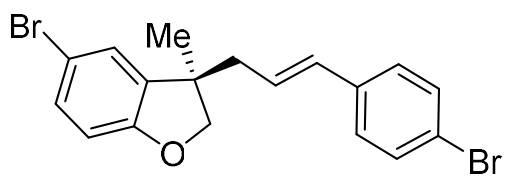

Chemical Formula: $\mathrm{C}_{18} \mathrm{H}_{16} \mathrm{Br}_{2} \mathrm{O}$

Exact Mass: 405.9568

3ek was prepared according to general procedure using $1 \mathrm{e}(0.1 \mathrm{mmol}, 1.0$ equiv, 34 $\mathrm{mg})$ and $2 \mathbf{k}(0.2 \mathrm{mmol}, 26.2 \mu \mathrm{L})$ and was purified by silica gel column chromatography (petroleum ether/EtOAc $=100 / 1 \sim 30 / 1)$ to obtain 3 ek as colorless oil $(18.4 \mathrm{mg}, 45 \%$ yield). ${ }^{1} \mathrm{H}$ NMR $\left(400 \mathrm{MHz}, \mathrm{CDCl}_{3}\right): \delta 7.49-7.38(\mathrm{~m}, 2 \mathrm{H}), 7.23(\mathrm{dd}, J=8.4, \mathrm{~Hz}, 1 \mathrm{H})$, 7.20-7.13 (m, 3H), $6.67(\mathrm{~d}, J=8.4 \mathrm{~Hz}, 1 \mathrm{H}), 6.50-6.26(\mathrm{~m}, 1 \mathrm{H}), 6.03$ (dt, $J=15.7,7.5$ $\mathrm{Hz}, 1 \mathrm{H}$ ), $4.44(\mathrm{~d}, J=8.8 \mathrm{~Hz}, 1 \mathrm{H}), 4.16(\mathrm{~d}, J=8.8 \mathrm{~Hz}, 1 \mathrm{H}$ ), 2.47 (ddd, $J=7.7,2.7,1.3$ $\mathrm{Hz}, 2 \mathrm{H}), 1.39$ (s, 3H);

${ }^{13} \mathrm{C}$ NMR (101 MHz, $\left.\mathrm{CDCl}_{3}\right): \delta$ 158.7, 137.2, 136.0, 132.7, 131.6, 131.1, 127.7, 126.0, $125.9,121.1,112.3,111.4,82.3,45.9,44.1,25.0$;

HRMS: (APCI) calcd for $\mathrm{C}_{18} \mathrm{H}_{17} \mathrm{Br}_{2} \mathrm{O}^{+}[\mathrm{M}+\mathrm{H}]^{+} 406.9641$; found 406.9639 .

The enantiomeric purity was established by HPLC analysis using a chiral column: OD$\mathrm{H}$ column, $30{ }^{\circ} \mathrm{C}, n$-Hexane/i-Propanol $=98 / 2$ as eluent, $254 \mathrm{~nm}, 0.5 \mathrm{~mL} / \mathrm{min}$. tR $=11.7$ $\min$ (minor), 12.9 min (major).

Optical Rotation: $[\alpha]_{D}^{28} 112.9$ (c $\left.0.4, \mathrm{CHCl}_{3}\right)$ for $90 \%$ ee. Absolute stereochemistry was determined through analogy with 3ac. 

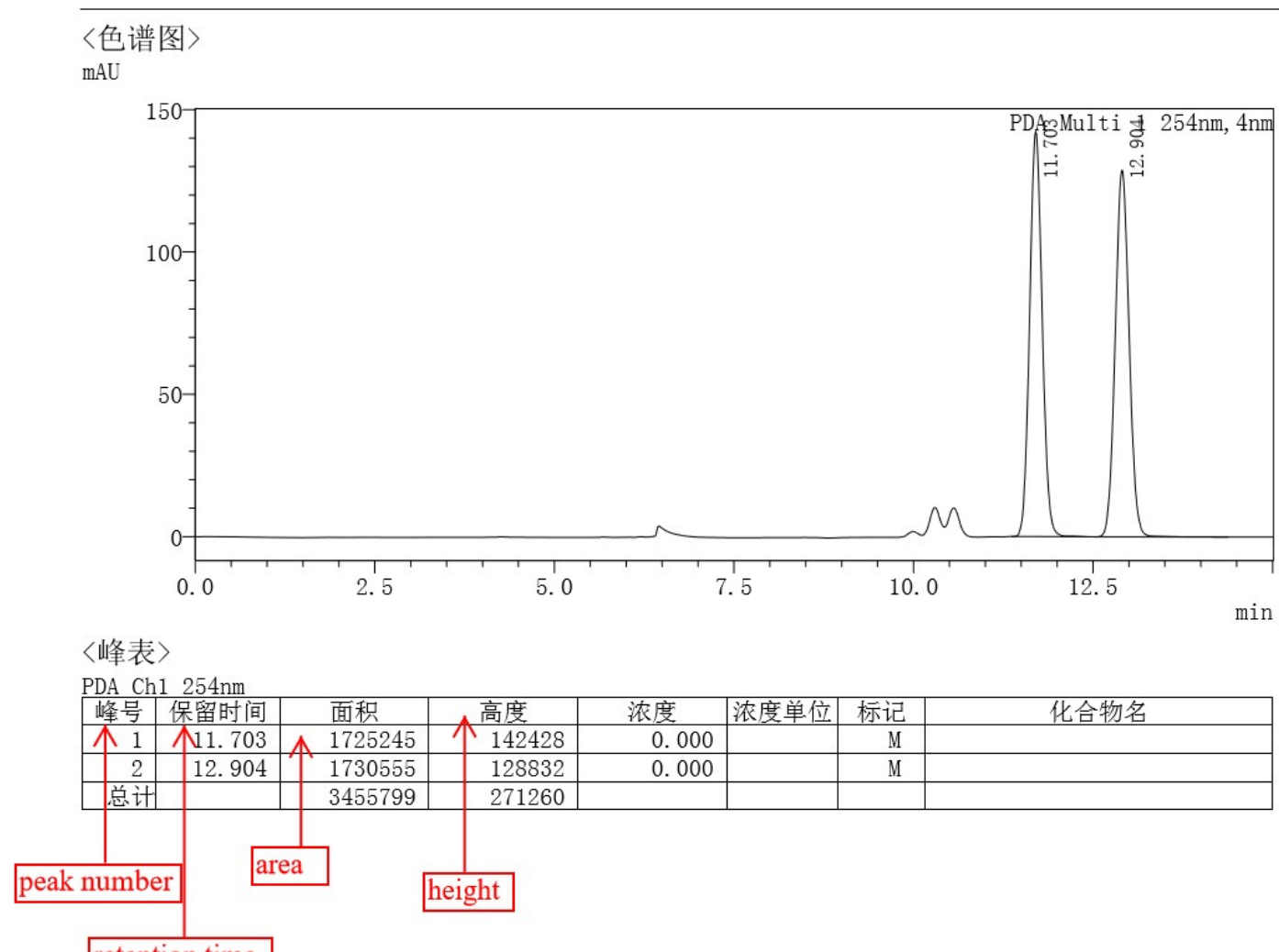

retention time

〈色谱图〉

$\mathrm{mAU}$

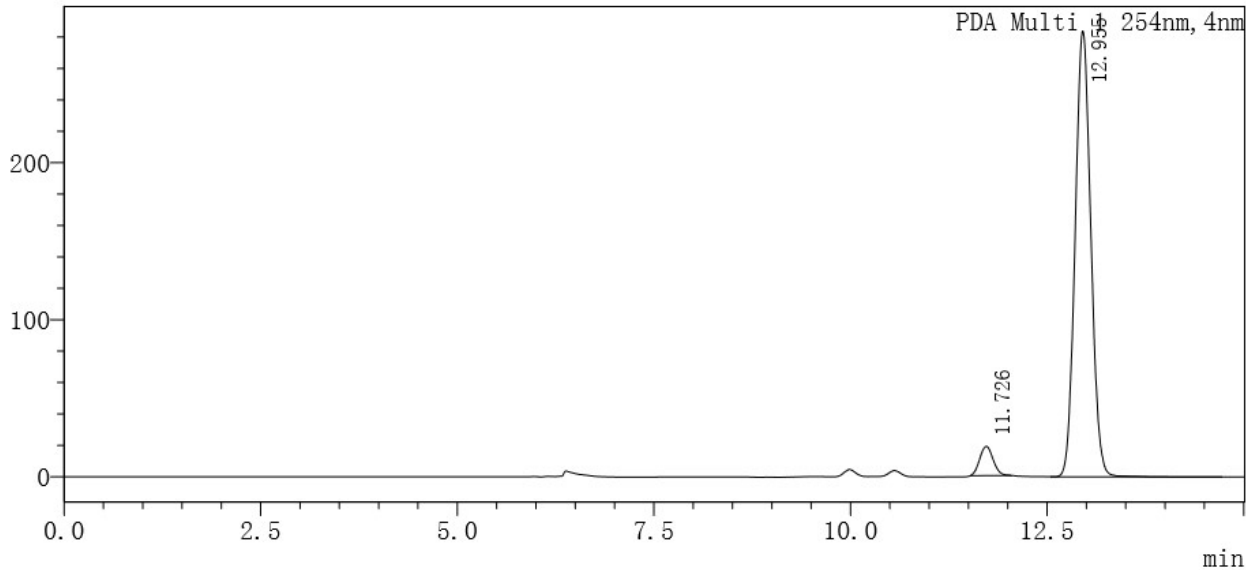

〈峰表〉

PDA Ch1 254nm

\begin{tabular}{|c|c|c|c|c|c|c|c|}
\hline 峰号 & 保留时间 & 面积 & 高度 & 浓度 & 浓度单位 & 标记 & 化合物名 \\
\hline 木1 1 & \$11.726 & 219604 & 18639 & 0.000 & & M & \\
\hline 2 & 12.955 & 3927873 & 283851 & 0.000 & & M & \\
\hline 总计 & & 4147478 & 302490 & & & & \\
\hline
\end{tabular}

retention time 
(R, E)-3-(3-(4-bromophenyl)allyl)-5-fluoro-3-methyl-2,3-dihydrobenzofuran (3fk)

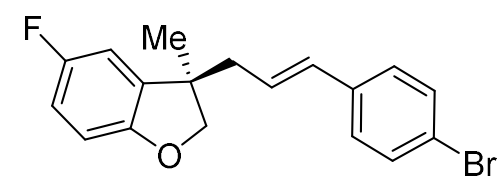

Chemical Formula: $\mathrm{C}_{18} \mathrm{H}_{16} \mathrm{BrFO}$

Exact Mass: 346.0369

3fk was prepared according to general procedure using $\mathbf{1 f}(0.1 \mathrm{mmol}, 1.0$ equiv, 28 $\mathrm{mg})$ and $\mathbf{2 k}(0.2 \mathrm{mmol}, 26.2 \mu \mathrm{L})$ and was purified by silica gel column chromatography (petroleum ether/EtOAc $=100 / 1 \sim 30 / 1)$ to obtain 3fk as colorless oil $(24.3 \mathrm{mg}, 70 \%$ yield). ${ }^{1} \mathrm{H}$ NMR (400 MHz, $\left.\mathrm{CDCl}_{3}\right): \delta$ 7.53-7.37 (m, 2H), 7.22-7.11 (m, 2H), 6.90-6.77 (m, 2H), 6.70 (ddd, $J=8.3,4.1,0.7 \mathrm{~Hz}, 1 \mathrm{H}), 6.41-6.29(\mathrm{~m}, 1 \mathrm{H}), 6.06(\mathrm{dt}, J=15.7,7.5$ $\mathrm{Hz}, 1 \mathrm{H}), 4.44(\mathrm{~d}, J=8.7 \mathrm{~Hz}, 1 \mathrm{H}), 4.17(\mathrm{~d}, J=8.7 \mathrm{~Hz}, 1 \mathrm{H}), 2.48(\mathrm{dt}, J=7.9,1.0 \mathrm{~Hz}$, 2H), 1.39 (s, 3H);

${ }^{13} \mathrm{C} \mathrm{NMR}\left(101 \mathrm{MHz}, \mathrm{CDCl}_{3}\right): \delta 157.6(\mathrm{~d}, J=237.4 \mathrm{~Hz}), 155.34,136.1,136.05,132.6$, 131.6, 127.7, 126.0, 121.1, 114.4 (d, $J=24.3 \mathrm{~Hz}), 109.98$ (d, J = 31.4 Hz), 109.9, 82.4, $46.1,44.0,24.8$;

${ }^{19} \mathrm{~F} \mathrm{NMR}\left(376 \mathrm{MHz}, \mathrm{CDCl}_{3}\right) \delta-123.77$;

HRMS: (APCI) calcd for $\mathrm{C}_{18} \mathrm{H}_{17} \mathrm{BrFO}^{+}[\mathrm{M}+\mathrm{H}]^{+} 347.0441$; found 347.0439 .

The enantiomeric purity was established by HPLC analysis using a chiral column: OD$\mathrm{H}$ column, $30^{\circ} \mathrm{C}, n$-Hexane $/ \mathrm{i}$-Propanol $=98 / 2$ as eluent, $254 \mathrm{~nm}, 0.5 \mathrm{~mL} / \mathrm{min}$. $\mathrm{tR}=11.2$ $\min$ (minor), 12.4 min (major).

Optical Rotation: $[\alpha]_{D}^{28} 12.4$ (c $\left.0.6, \mathrm{CHCl}_{3}\right)$ for $91 \%$ ee.

Absolute stereochemistry was determined through analogy with 3ac. 
〈色谱图〉

mAU

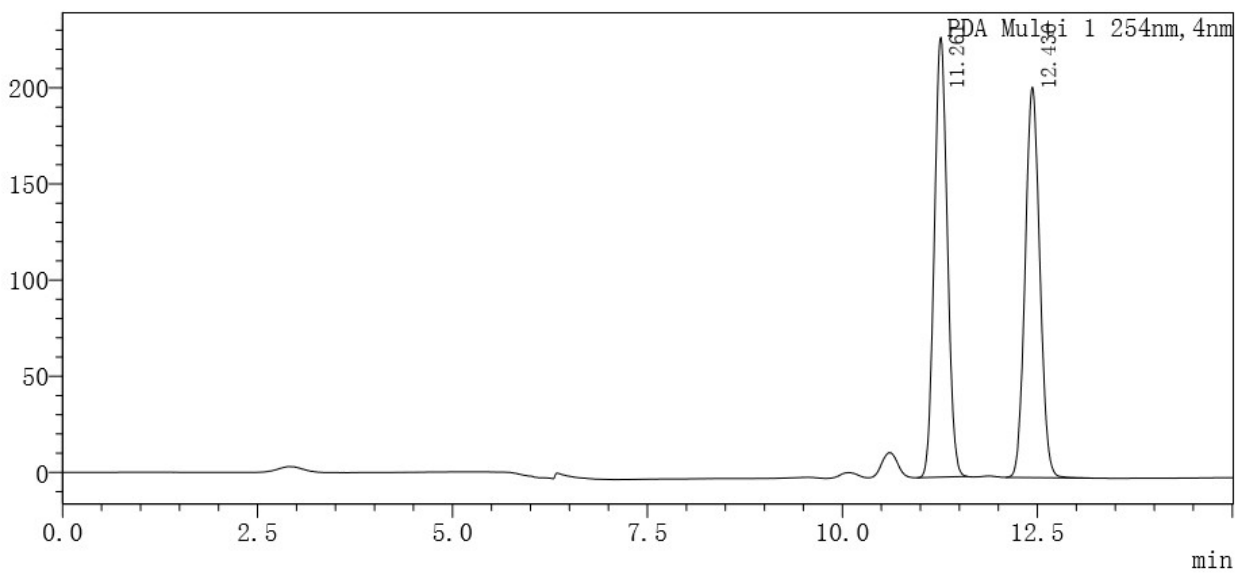

〈峰表〉

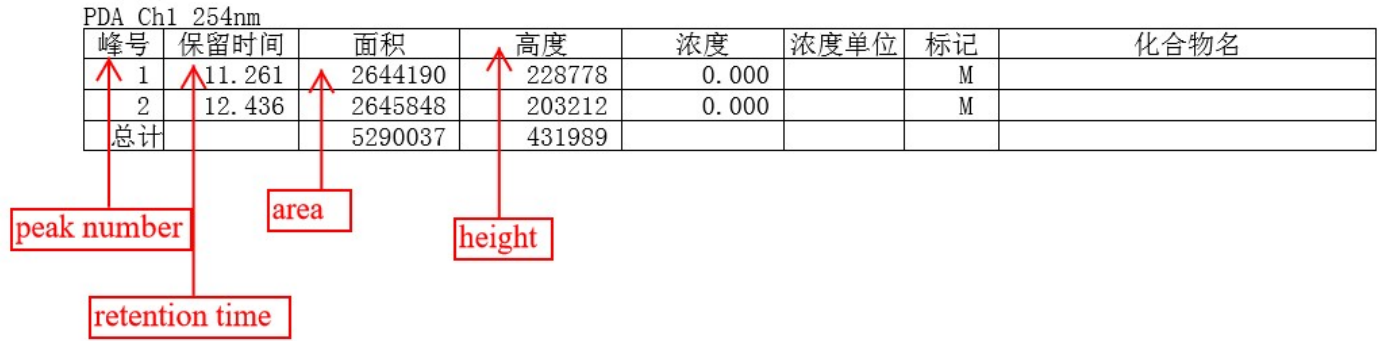

〈色谱图〉

$\mathrm{mAU}$

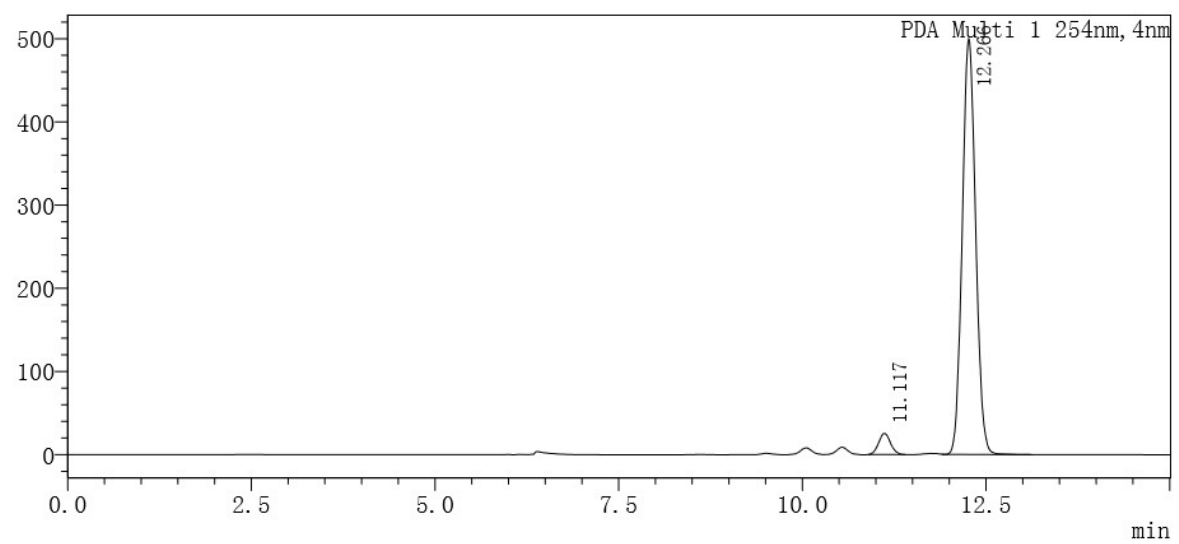

〈峰表〉

\begin{tabular}{|c|c|c|c|c|c|c|c|}
\hline 峰号 & 保留时间 & 面积 & 高度 & 浓度 & 浓度单位 & 标记 & 化合物名 \\
\hline 11 & A 11.117 & 284317 & 25505 & 0.000 & & M & \\
\hline 2 & 12.266 & 6317892 & 500394 & 0.000 & & M & \\
\hline 总计 & & 6602209 & 525899 & & & & \\
\hline
\end{tabular}

retention time 
(R, E)-3-(3-(4-bromophenyl)allyl)-3,6-dimethyl-2,3-dihydrobenzofuran (3gk)

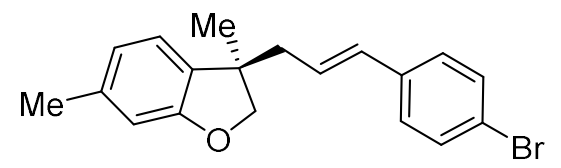

Chemical Formula: $\mathrm{C}_{19} \mathrm{H}_{19} \mathrm{BrO}$

Exact Mass: 342.0619

3gk was prepared according to general procedure using $1 \mathbf{g}(0.1 \mathrm{mmol}, 1.0$ equiv, 27.6 $\mathrm{mg})$ and $\mathbf{2 k}(0.2 \mathrm{mmol}, 26.2 \mu \mathrm{L})$ and was purified by silica gel column chromatography (petroleum ether/EtOAc $=100 / 1 \sim 30 / 1)$ to obtain $3 \mathbf{g k}$ as colorless oil $(28.1 \mathrm{mg}, 82 \%$ yield). ${ }^{1} \mathrm{H}$ NMR (400 MHz, $\left.\mathrm{CDCl}_{3}\right): \delta$ 7.49-7.36 (m, 2H), 7.23-7.14 (m, 2H), 6.99 (d, J = $7.5 \mathrm{~Hz}, 1 \mathrm{H}), 6.72$ (ddd, $J=7.5,1.5,0.8 \mathrm{~Hz}, 1 \mathrm{H}), 6.64(\mathrm{dt}, J=1.5,0.7 \mathrm{~Hz}, 1 \mathrm{H}), 6.34$ (dd, $J=15.7,1.5 \mathrm{~Hz}, 1 \mathrm{H}), 6.10(\mathrm{dt}, J=15.7,7.5 \mathrm{~Hz}, 1 \mathrm{H}), 4.41(\mathrm{~d}, J=8.7 \mathrm{~Hz}, 1 \mathrm{H}), 4.15$ (d, $J=8.7 \mathrm{~Hz}, 1 \mathrm{H}), 2.48(\mathrm{dd}, J=7.5,1.3 \mathrm{~Hz}, 2 \mathrm{H}), 2.33(\mathrm{~d}, J=0.8 \mathrm{~Hz}, 3 \mathrm{H}), 1.38(\mathrm{~s}, 3 \mathrm{H})$; ${ }^{13} \mathrm{C}$ NMR $\left(101 \mathrm{MHz}, \mathrm{CDCl}_{3}\right): \delta 159.7,138.4,136.3,13,131.7,131.6,127.7,126.8$, $122.5,121.2,120.9,110.4,82.2,45.3,44.3,25.0,21.5$

HRMS: (APCI) calcd for $\mathrm{C}_{19} \mathrm{H}_{20} \mathrm{BrO}^{+}[\mathrm{M}+\mathrm{H}]^{+} 343.0692$; found 343.0696.

The enantiomeric purity was established by HPLC analysis using a chiral column: OJ$\mathrm{H}$ column, $30{ }^{\circ} \mathrm{C}, n$-Hexane/i-Propanol $=95 / 5$ as eluent, $254 \mathrm{~nm}, 1 \mathrm{~mL} / \mathrm{min}$. $\mathrm{tR}=8.4$ $\min$ (minor), 14.1 min (major).

Optical Rotation: $[\alpha]_{D}{ }^{30}-7.2\left(\mathrm{c} 0.7, \mathrm{CHCl}_{3}\right)$ for $91 \%$ ee. Absolute stereochemistry was determined through analogy with 3ac. 
〈色谱图〉

mAU

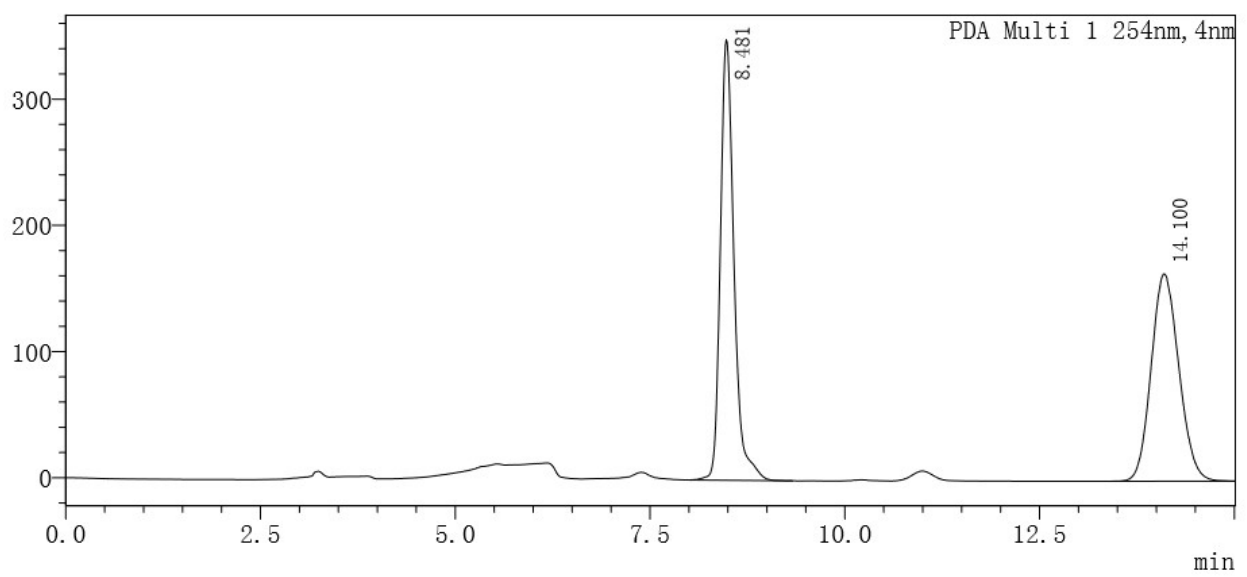

〈峰表〉

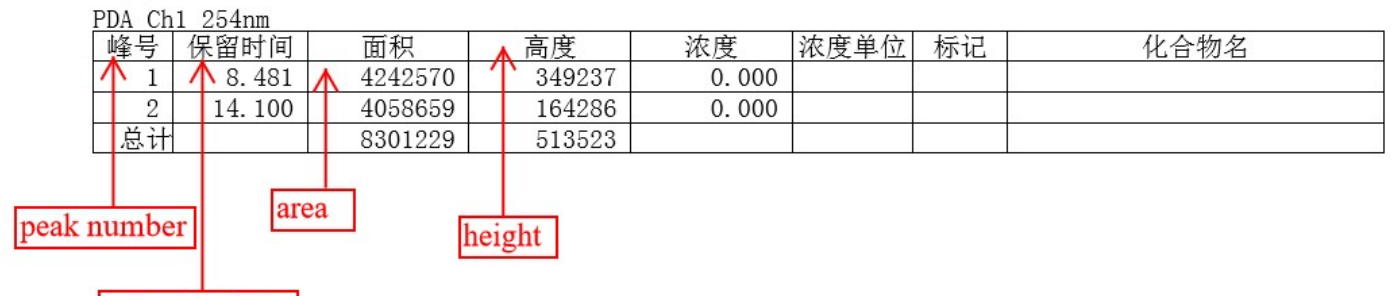

retention time

〈色谱图〉

$\mathrm{mAU}$

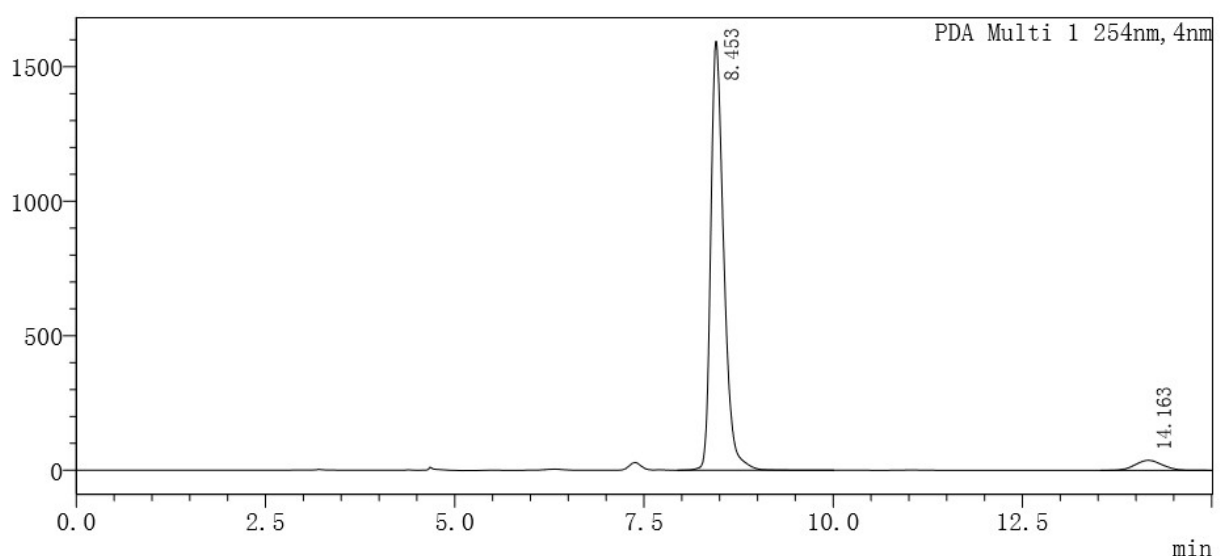

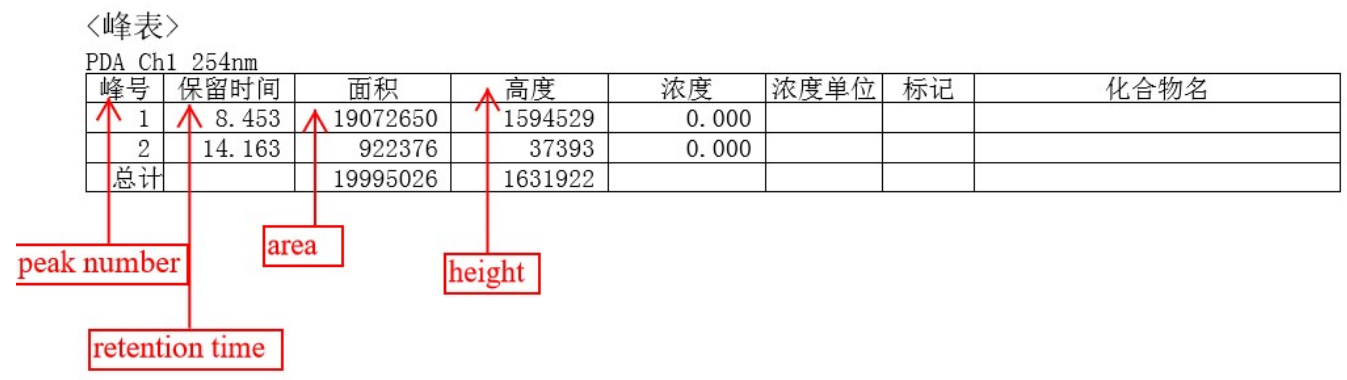


(R, E)-3-(3-(4-bromophenyl)allyl)-6-chloro-3-methyl-2,3-dihydrobenzofuran (3hk)

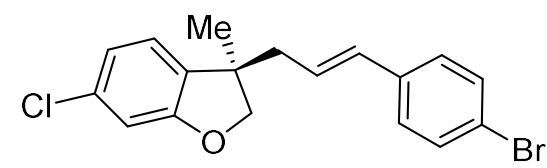

Chemical Formula: $\mathrm{C}_{18} \mathrm{H}_{16} \mathrm{BrClO}$

Exact Mass: 362.0073

3hk was prepared according to general procedure using $1 \mathrm{~h}(0.1 \mathrm{mmol}, 1.0$ equiv, 29.6 $\mathrm{mg})$ and $2 \mathbf{k}(0.2 \mathrm{mmol}, 26.2 \mu \mathrm{L})$ and was purified by silica gel column chromatography (petroleum ether/EtOAc $=100 / 1 \sim 30 / 1)$ to obtain $3 \mathbf{h k}$ as colorless oil $(24.3 \mathrm{mg}, 67 \%$ yield). ${ }^{1} \mathrm{H}$ NMR (400 MHz, $\left.\mathrm{CDCl}_{3}\right): \delta 7.54-7.37(\mathrm{~m}, 2 \mathrm{H}), 7.16(\mathrm{~d}, J=8.4 \mathrm{~Hz}, 2 \mathrm{H}), 6.99$ $(\mathrm{d}, J=7.9 \mathrm{~Hz}, 1 \mathrm{H}), 6.87(\mathrm{dd}, J=7.9,1.8 \mathrm{~Hz}, 1 \mathrm{H}), 6.79(\mathrm{~d}, J=1.8 \mathrm{~Hz}, 1 \mathrm{H}), 6.33(\mathrm{dt}, J$ $=15.7,1.4 \mathrm{~Hz}, 1 \mathrm{H}), 6.05(\mathrm{dt}, J=15.7,7.5 \mathrm{~Hz}, 1 \mathrm{H}), 4.45(\mathrm{~d}, J=8.7 \mathrm{~Hz}, 1 \mathrm{H}), 4.18(\mathrm{~d}, J$ $=8.8 \mathrm{~Hz}, 1 \mathrm{H}), 2.47(\mathrm{dt}, J=7.8,1.3 \mathrm{~Hz}, 2 \mathrm{H}), 1.38(\mathrm{~s}, 3 \mathrm{H})$;

${ }^{13} \mathrm{C}$ NMR $\left(101 \mathrm{MHz}, \mathrm{CDCl}_{3}\right): \delta$ 160.4, 136.0, 133.5, 133.4, 132.6, 131.6, 127.7, 126.0, $123.5,121.1,120.7,110.5,82.7,45.3,44.2,25.0$;

HRMS: (APCl) calcd for $\mathrm{C}_{18} \mathrm{H}_{17} \mathrm{BrClO}^{+}[\mathrm{M}+\mathrm{H}]^{+}$363.0146; found 363.0144.

The enantiomeric purity was established by HPLC analysis using a chiral column: AD$\mathrm{H}$ column, $30{ }^{\circ} \mathrm{C}, n$-Hexane/i-Propanol $=97 / 3$ as eluent, $254 \mathrm{~nm}, 1 \mathrm{~mL} / \mathrm{min}$. $\mathrm{tR}=5.3$ $\min$ (minor), $5.6 \min$ (major).

Optical Rotation: $[\alpha]_{\triangleright}{ }^{30}-8.6\left(\mathrm{c} 0.7, \mathrm{CHCl}_{3}\right)$ for $94 \%$ ee.

Absolute stereochemistry was determined through analogy with 3ac. 
〈色谱图〉

$\mathrm{mAU}$

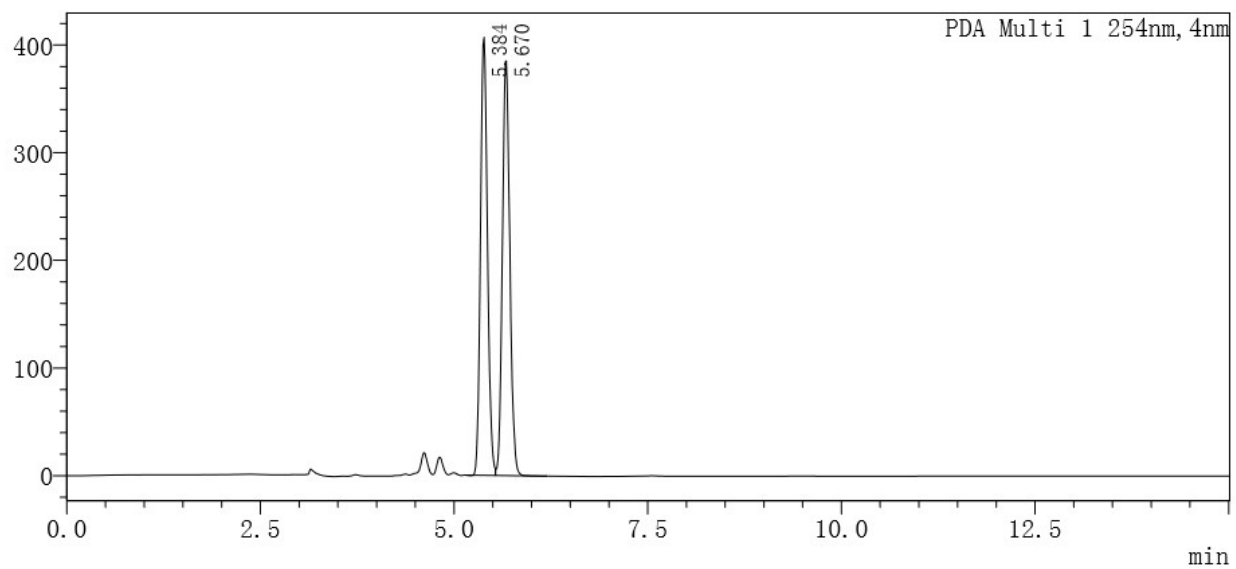

〈峰表〉

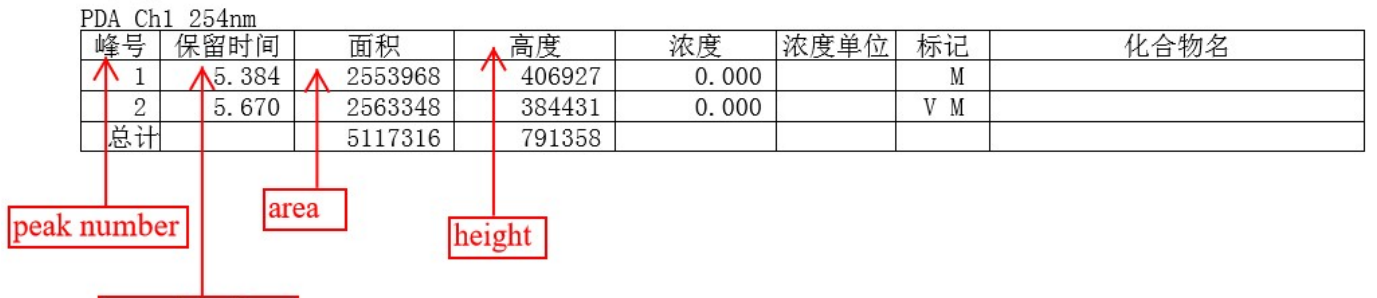

retention time

〈色谱图〉

$\mathrm{mAU}$

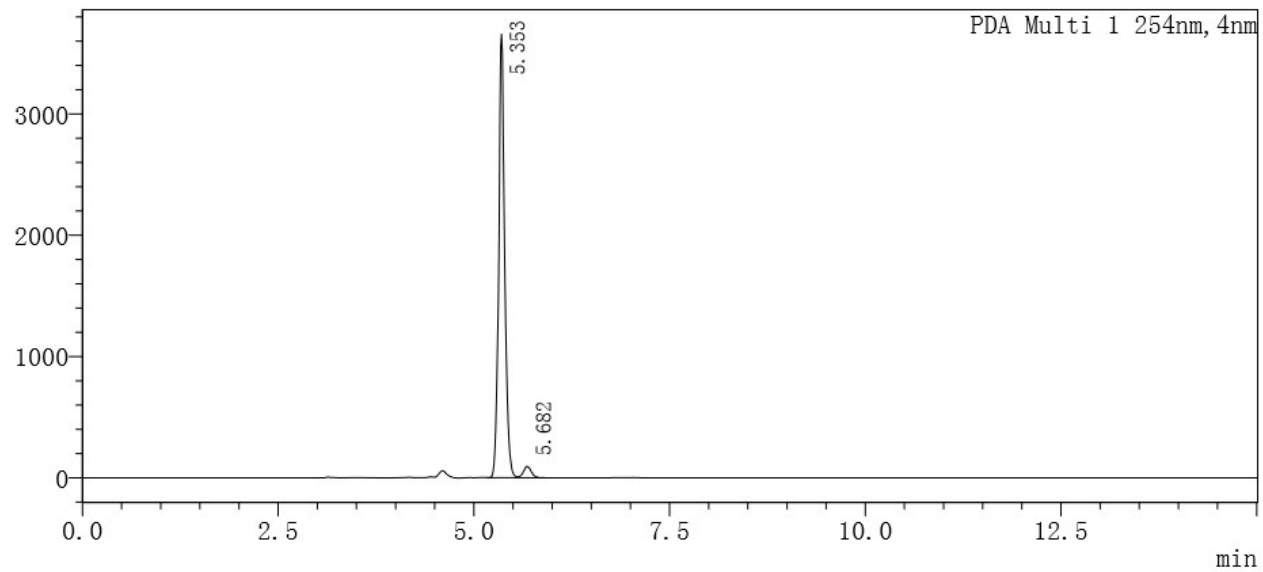

〈峰表〉

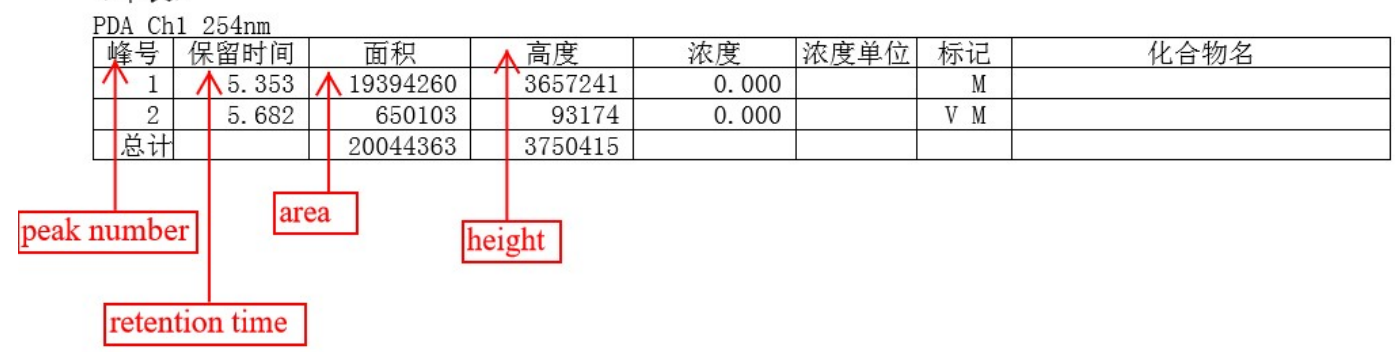


(3R)-3-methyl-3-((3-phenyloxiran-2-yl)methyl)-2,3-dihydrobenzofuran (4)

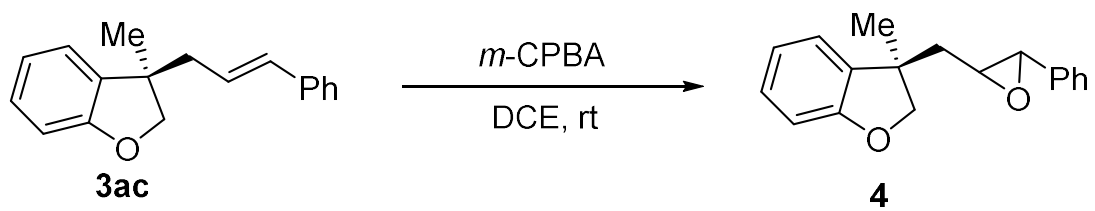

To a stirred solution of $3 \mathrm{ac}(0.4 \mathrm{mmol}, 120.1 \mathrm{mg})$ in DCE $(2.0 \mathrm{~mL})$ was added $m$-CPBA $(0.48 \mathrm{mmol}, 84.0 \mathrm{mg})$ in three pots. The mixture was then stirred at room temperature for 24 hours. After the reaction was completed, the solid was filtrated and the solvent was removed under vacuum. The residue was then purified by column chromatography on silica gel, eluting with petroleum ether/EtOAc $=50 / 1 \sim 20 / 1$ to afford the desired epoxide $4(85.2 \mathrm{mg}, 80 \%$ yield, d.r. $=1.1 / 1)$.

${ }^{1} \mathrm{H}$ NMR $\left(400 \mathrm{MHz}, \mathrm{CDCl}_{3}\right):$ 7.41-7.21 (m, 8H), 7.20-7.05 (m, 6H), 6.97-6.87 (m, 2H), 6.85-6.73 (m, 2H), $4.60(\mathrm{~d}, J=8.8 \mathrm{~Hz}, 1 \mathrm{H}), 4.51(\mathrm{~d}, J=8.8 \mathrm{~Hz}, 1 \mathrm{H}), 4.25(\mathrm{~d}, J=1.1$ $\mathrm{Hz}, 1 \mathrm{H}), 4.23(\mathrm{~d}, J=1.2 \mathrm{~Hz}, 1 \mathrm{H}), 3.55(\mathrm{~d}, J=\mathrm{Hz}, 1 \mathrm{H}), 3.44(\mathrm{~d}, J=\mathrm{Hz}, 1 \mathrm{H}), 3.05-$ $2.94(\mathrm{~m}, 1 \mathrm{H}), 2.93-2.83(\mathrm{~m}, 1 \mathrm{H}), 0-1.90(\mathrm{~m}, 4 \mathrm{H}), 1.49(\mathrm{~s}, 3 \mathrm{H}), 1.47(\mathrm{~s}, 3 \mathrm{H})$;

${ }^{13} \mathrm{C} \mathrm{NMR}\left(100 \mathrm{MHz}, \mathrm{CDCl}_{3}\right): \delta$ 159.5, 159.2, 137.2, 137.1, 134.4, 133.9, 128.4, 128.4, 128.3, 128.1, 127.9, 125.5, 125.4, 122.97, 122.7, 120.7, 120.6, 109.9, 109.9, 82.8, 81.8, 59.9, 59.8, 58.6, 58.1, 44.7, 44.6, 43.6, 42.9, 26.1, 24.6;

HRMS: (ESI) calcd for $\mathrm{C}_{18} \mathrm{H}_{18} \mathrm{NaO}_{2}{ }^{+}[\mathrm{M}+\mathrm{Na}]^{+} 289.1199$; found 289.1201. 
(2aR,4R,5R)-2a-methyl-5-phenyl-2a,3,4,5-tetrahydro-2H-naphtho[1,8-bc]furan-4-ol (5)

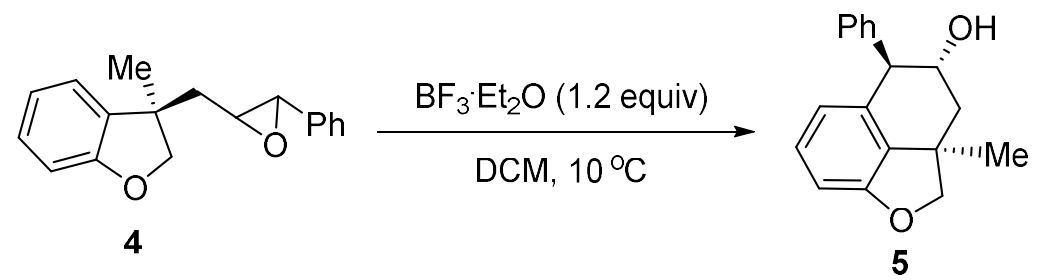

To a solution of $4(0.1 \mathrm{mmol}, 26.6 \mathrm{mg})$ in dry $\mathrm{DCM}(2 \mathrm{~mL})$ at $10{ }^{\circ} \mathrm{C}$ under an argon atmosphere was added $\mathrm{BF}_{3} \cdot \mathrm{Et}_{2} \mathrm{O}(0.12 \mathrm{mmol})$ slowly. After the reaction was completed, the reaction was quenched by the addition of saturated aqueous $\mathrm{NaHCO}_{3}$ and extracted with DCM. The combined organic layers was washed with satuated $\mathrm{NH}_{4} \mathrm{Cl}$ and dried over $\mathrm{Na}_{2} \mathrm{SO}_{4}$. The solvent was then removed under vacuum and the residue was purified by column chromatography on silica gel, eluting with petroleum ether/EtOAc $=20: 1 \sim 5 / 1$ to afford the desired tricyclic dihydrobenzofuran 5 (16.0 mg, $60 \%)$ with d.r. (100/13).

${ }^{1} \mathrm{H}$ NMR $\left(400 \mathrm{MHz}, \mathrm{CDCl}_{3}\right):$ $7.47-7.40(\mathrm{~m}, 2 \mathrm{H}), 7.39-7.32(\mathrm{~m}, 1 \mathrm{H}), 7.31-7.24(\mathrm{~m}, 2 \mathrm{H})$, $6.99(\mathrm{t}, J=7.8 \mathrm{~Hz}, 1 \mathrm{H}), 6.72-6.67(\mathrm{~m}, 1 \mathrm{H}), 6.23-6.17(\mathrm{~m}, 1 \mathrm{H}), 4.50(\mathrm{~d}, J=8.2 \mathrm{~Hz}, 1 \mathrm{H})$, $4.22(\mathrm{~d}, J=8.1 \mathrm{~Hz}, 1 \mathrm{H}), 4.18-4.08(\mathrm{~m}, 1 \mathrm{H}), 3.94(\mathrm{~d}, J=8.9 \mathrm{~Hz}, 1 \mathrm{H}), 2.33(\mathrm{dd}, J=14.2$, $8.0 \mathrm{~Hz}, 1 \mathrm{H}), 1.99$ (dd, J = 14.1, $6.8 \mathrm{~Hz}, 1 \mathrm{H}), 1.74$ (bs, 1H), 1.45 (s, 3H);

${ }^{13} \mathrm{C} \mathrm{NMR}\left(100 \mathrm{MHz}, \mathrm{CDCl}_{3}\right): \delta 157.1,139.2,136.2,132.6,129.7,129.0,128.3,127.5$, $118.5,107.7,87.1,75.1,5,41.0,40.3,27.7$;

HRMS: (ESI) calcd for $\mathrm{C}_{18} \mathrm{H}_{18} \mathrm{NaO}_{2}{ }^{+}[\mathrm{M}+\mathrm{Na}]^{+} 289.1199$; found 289.1201.

Optical Rotation: $[\alpha]_{\mathrm{D}}{ }^{30} 23.0$ (c $\left.0.3, \mathrm{CHCl}_{3}\right)$. 


\section{Copies of the ${ }^{1} \mathrm{H},{ }^{13} \mathrm{C}$ and ${ }^{19} \mathrm{~F}$ NMR spectra}

\section{3aa}

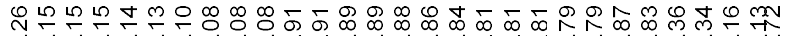

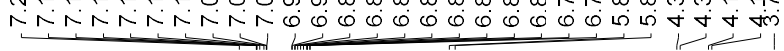

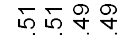

กั่ $ง$

$\stackrel{\infty}{m}$
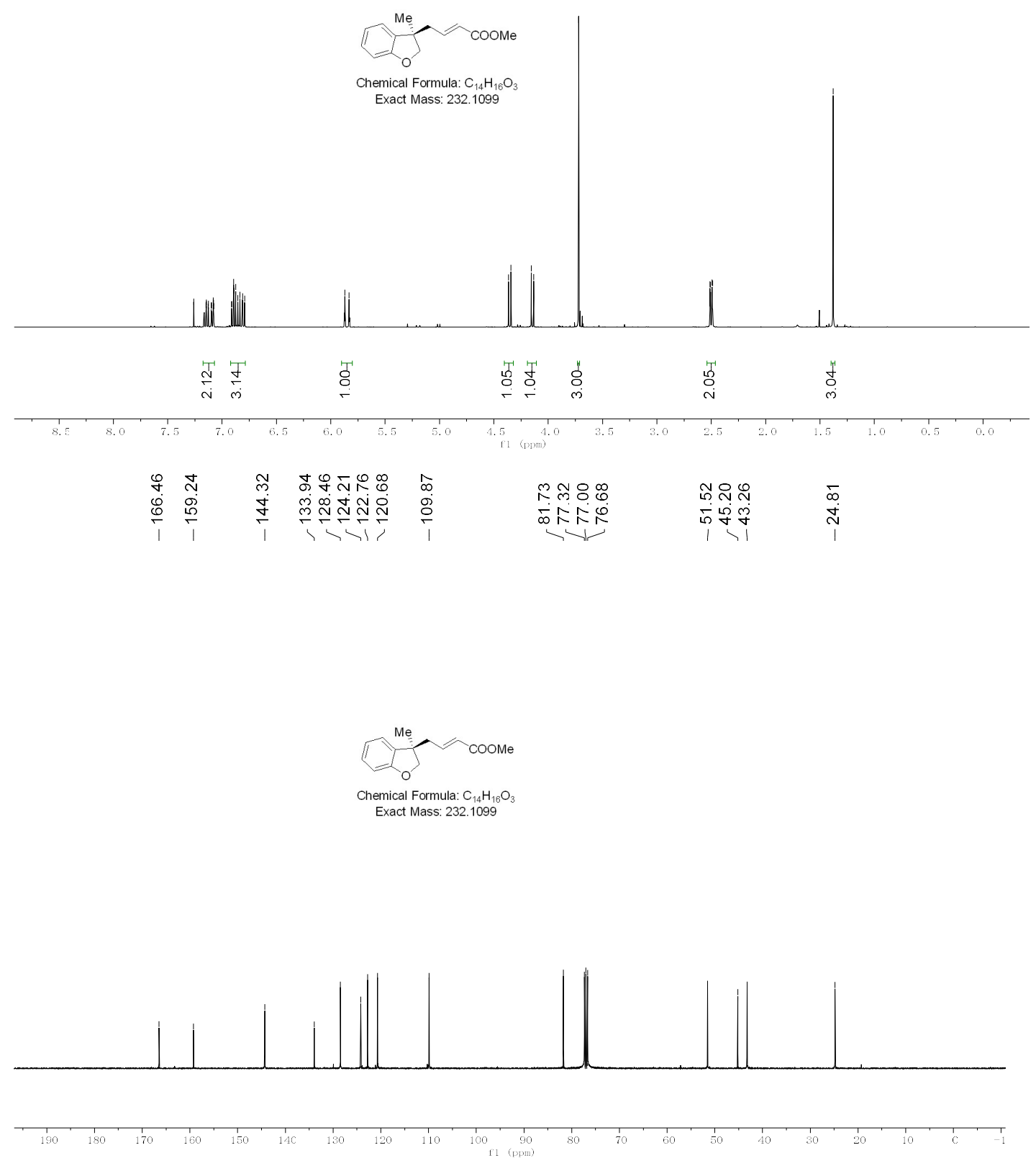
$3 a b$

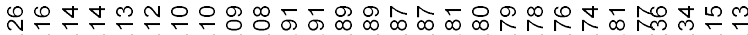

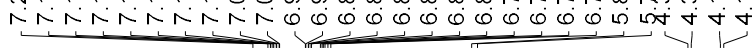

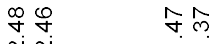

$\stackrel{n}{\circ}$

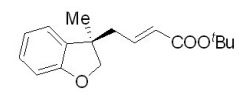

Chemical Formula: $\mathrm{C}_{17} \mathrm{H}_{22} \mathrm{O}$ Exact Mass: 274.1569

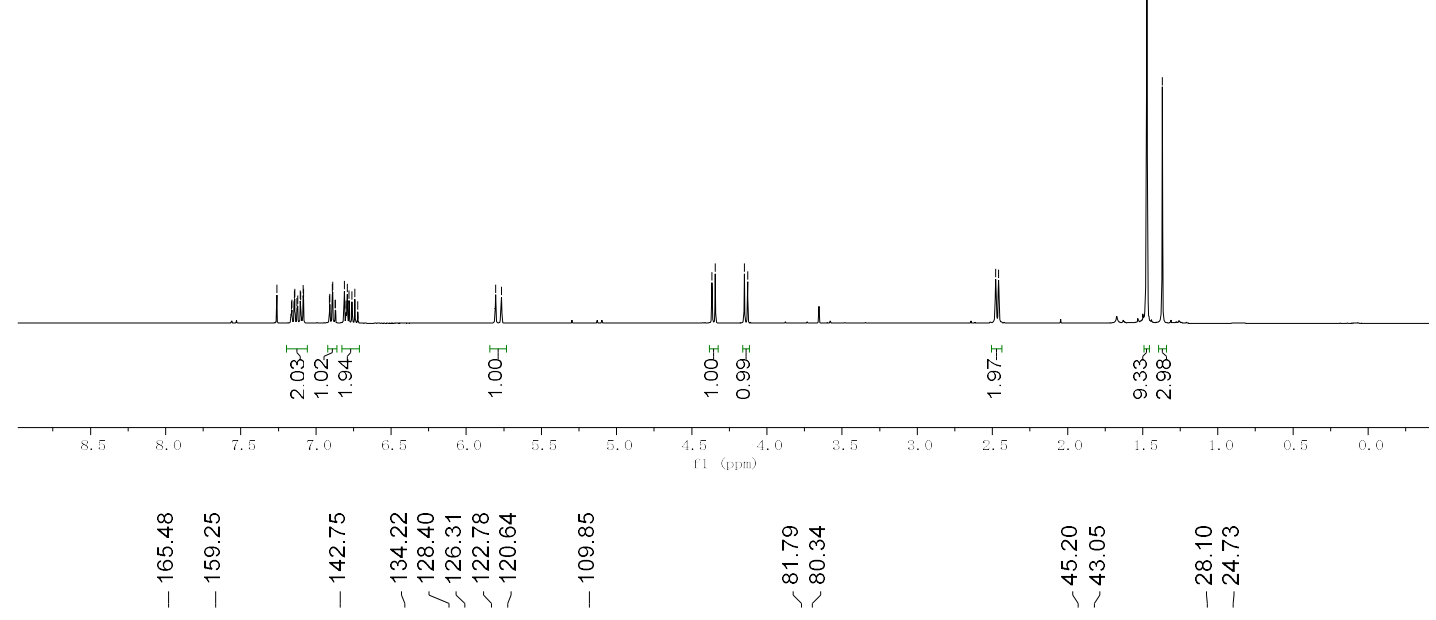

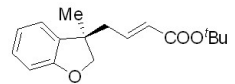

Chemical Formula: $\mathrm{C}_{17} \mathrm{H}_{22} \mathrm{O}_{3}$

Exact Mass: 274.1569
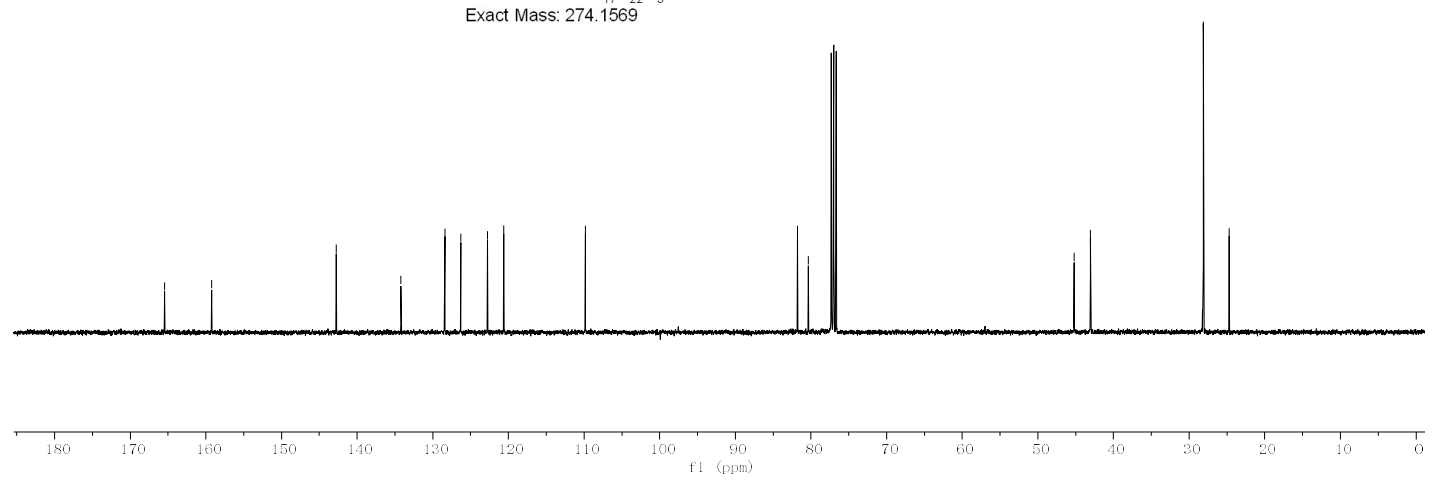
3ac

림ำ

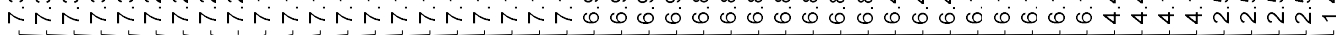
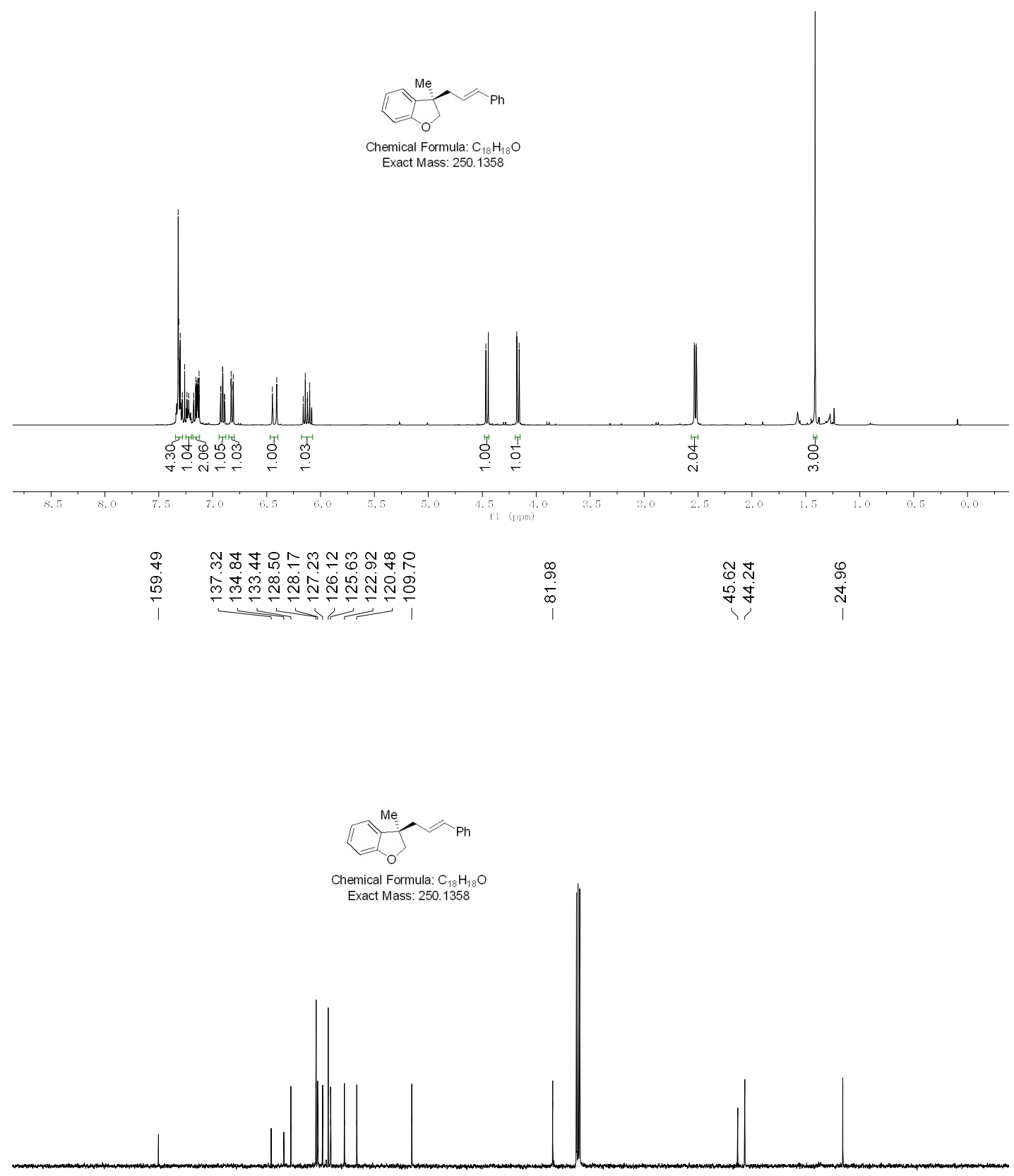
3ad

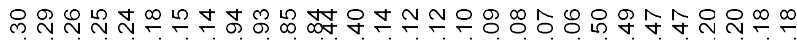

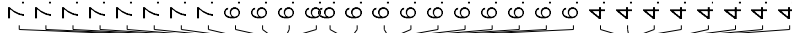

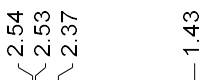
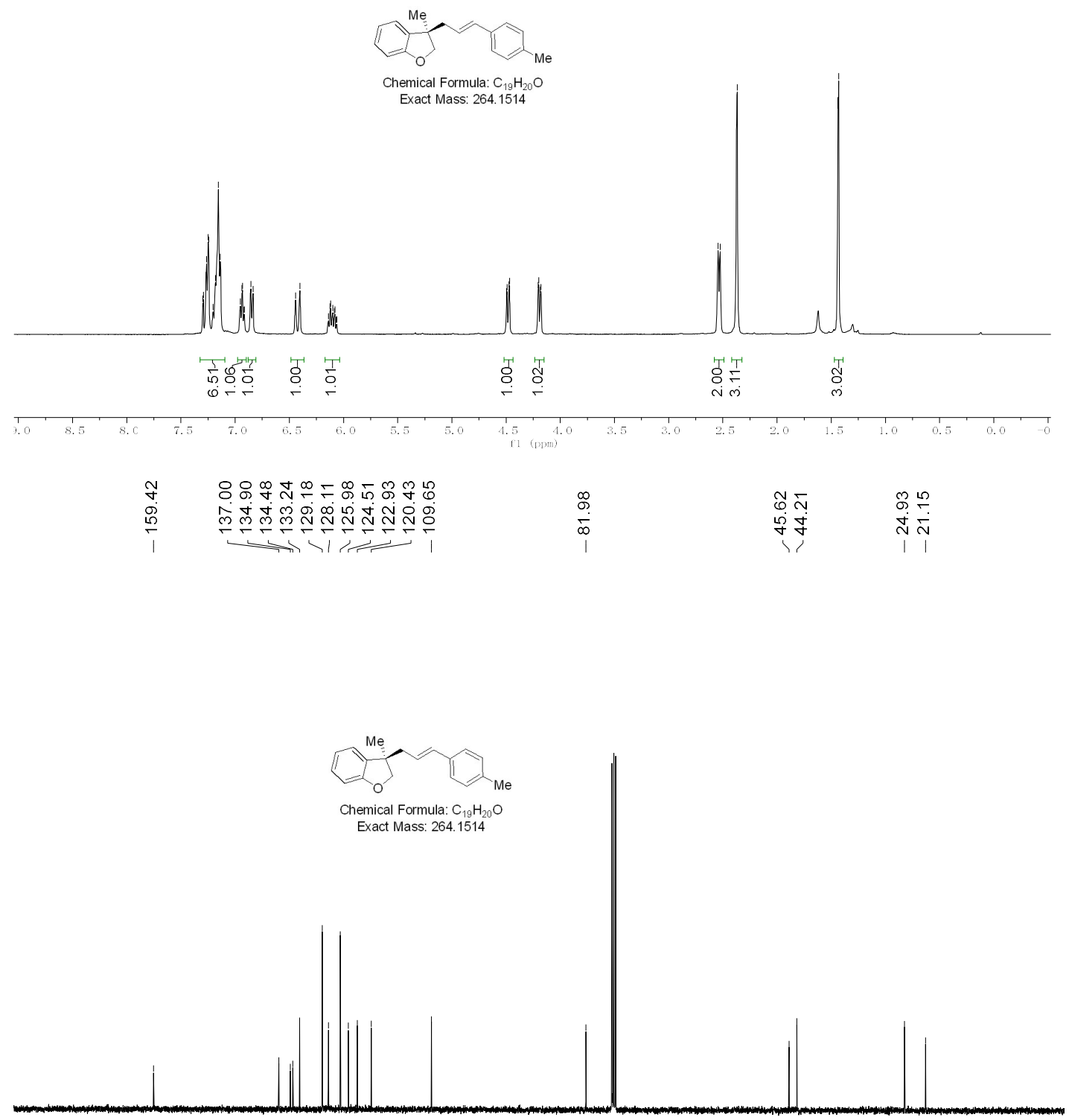
3ae

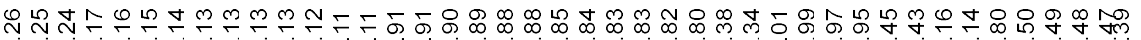

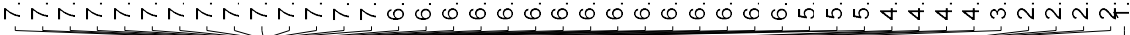
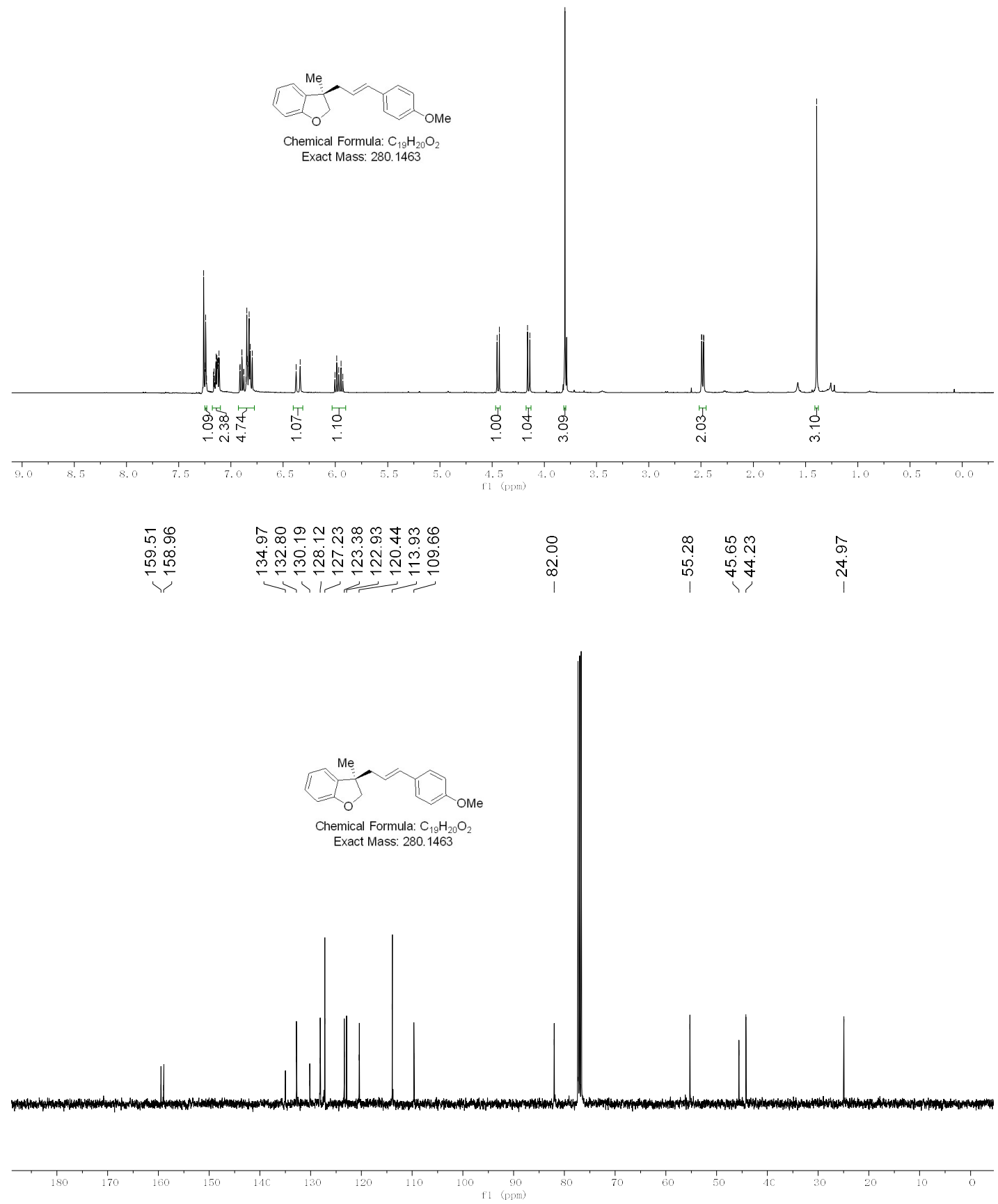
3af

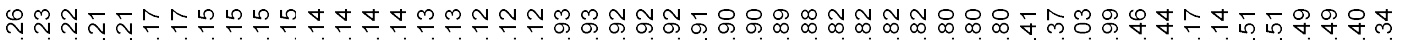

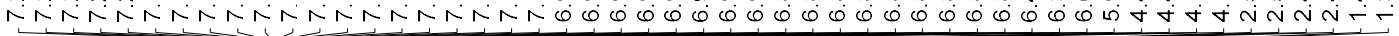
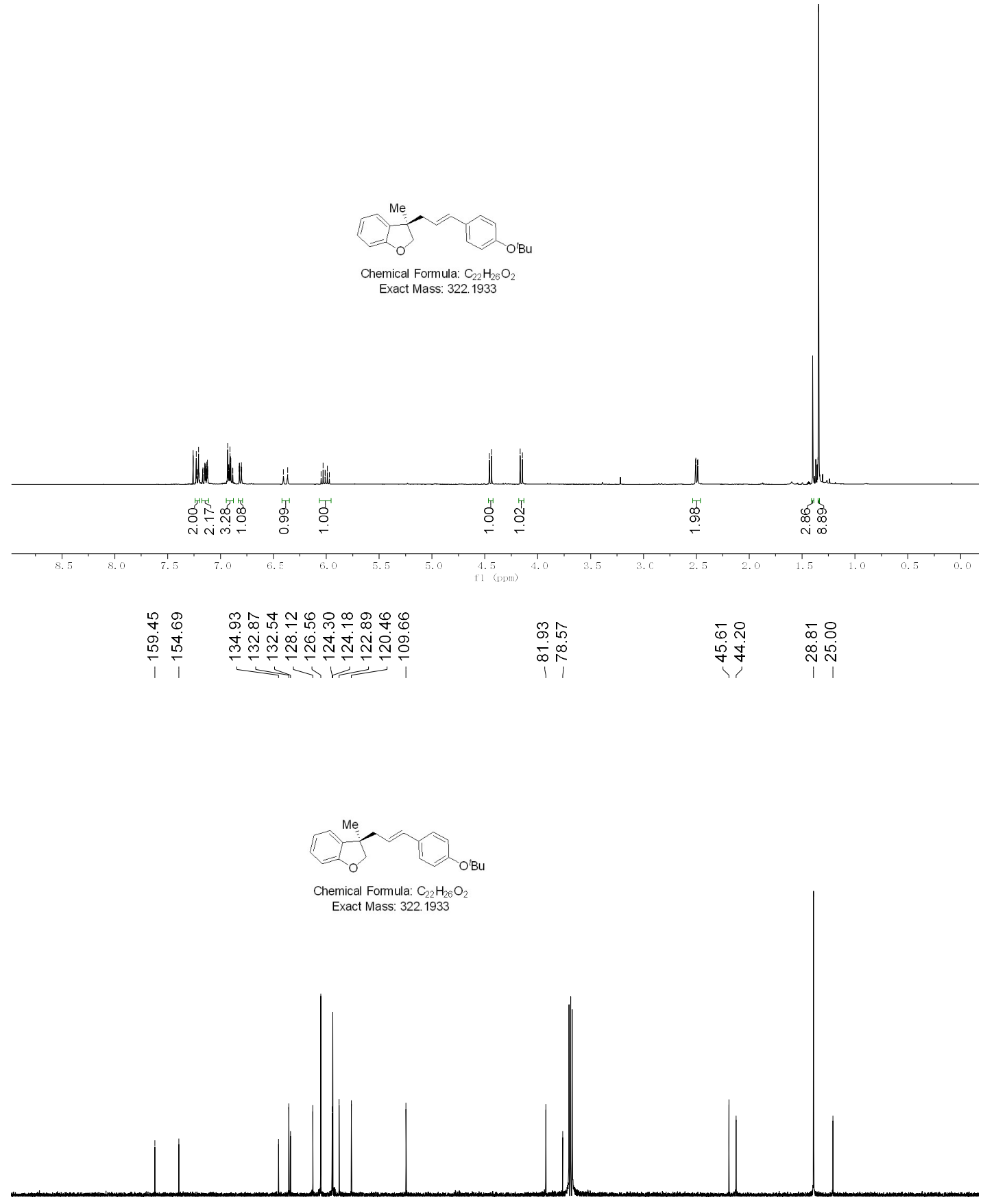

ᄂ) 8 का

Г্

i

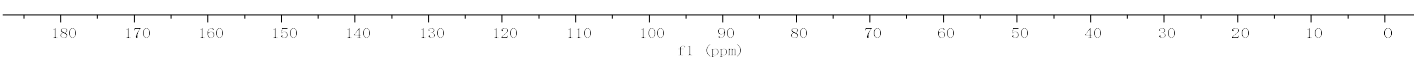


3ag

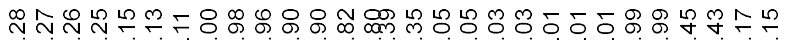

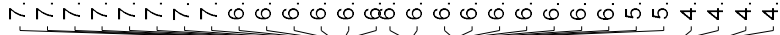

हु

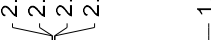
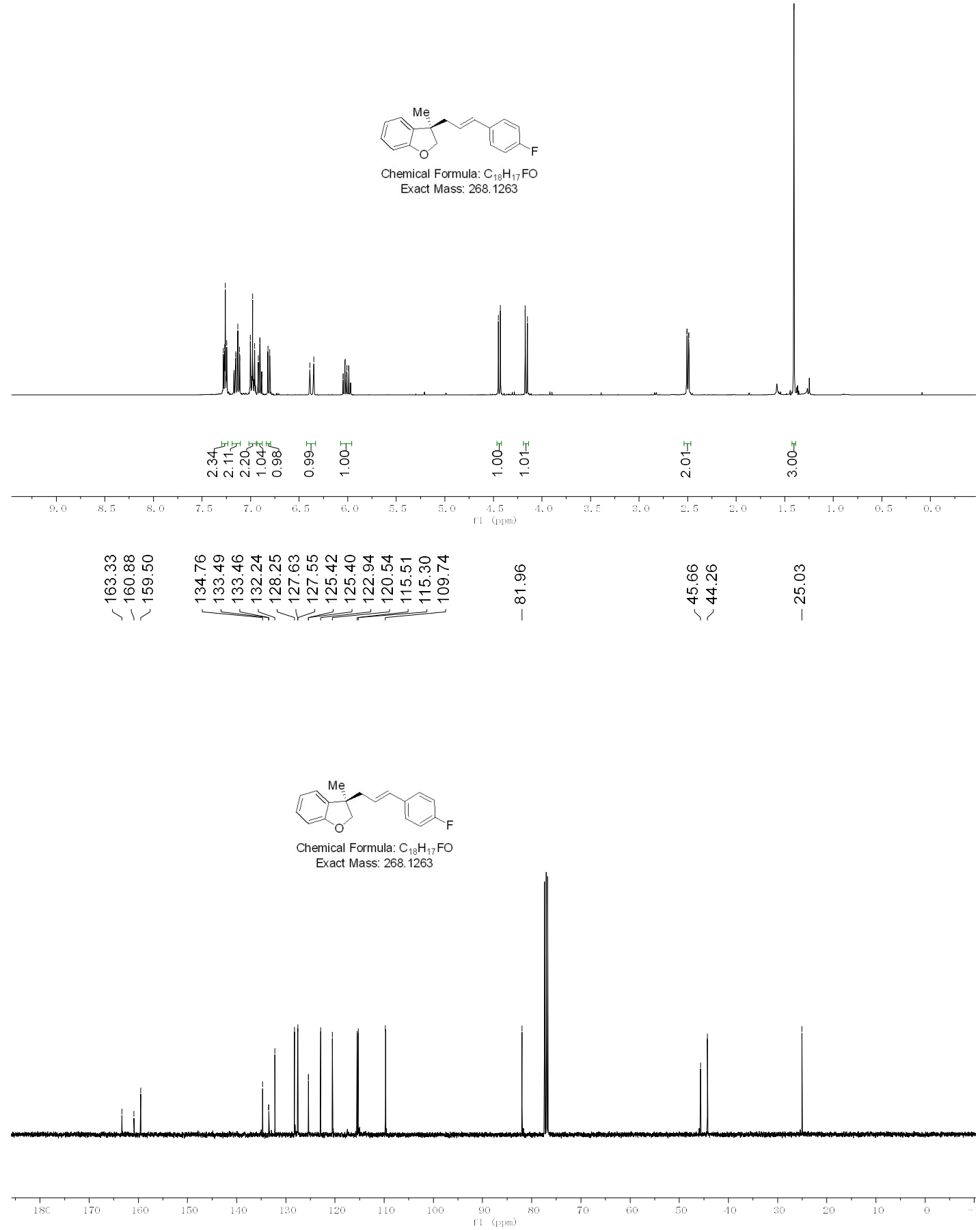


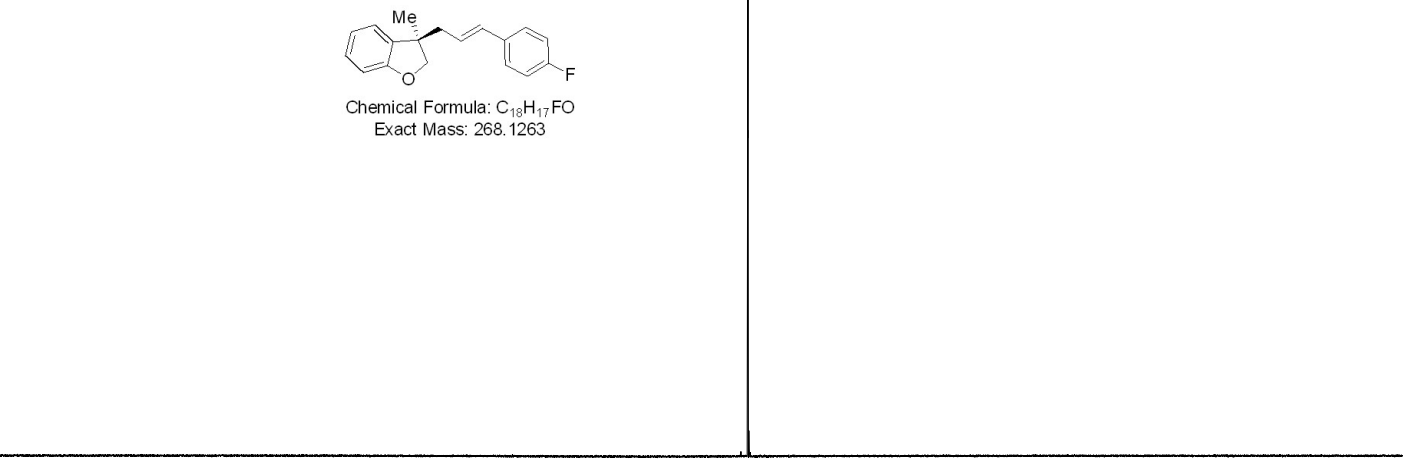

Chemical Formula: $\mathrm{C}_{18} \mathrm{H}_{12} \mathrm{FO}$ Exact Mass: 268.1263

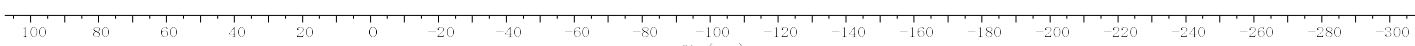

$\mathrm{f1}$ (pim) 
3ah

음 암

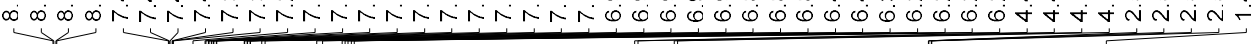
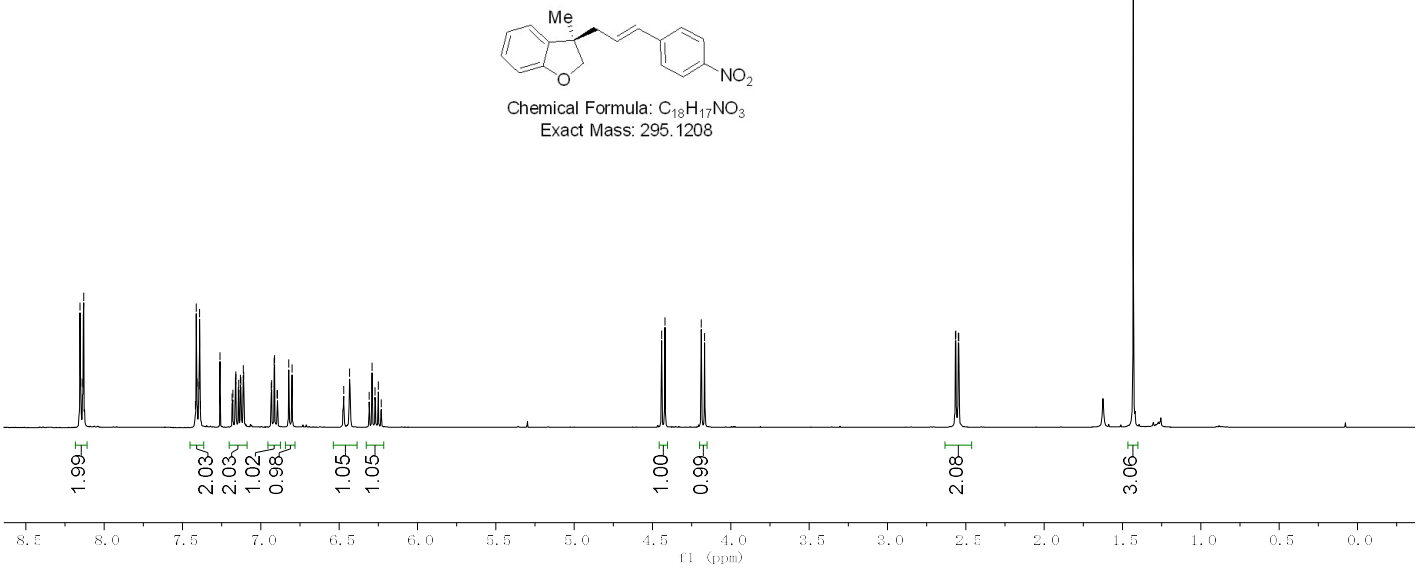

\& $\quad$ C

帛

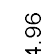

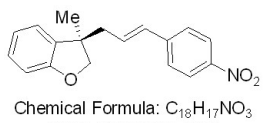
Exact Mass: 295.1208

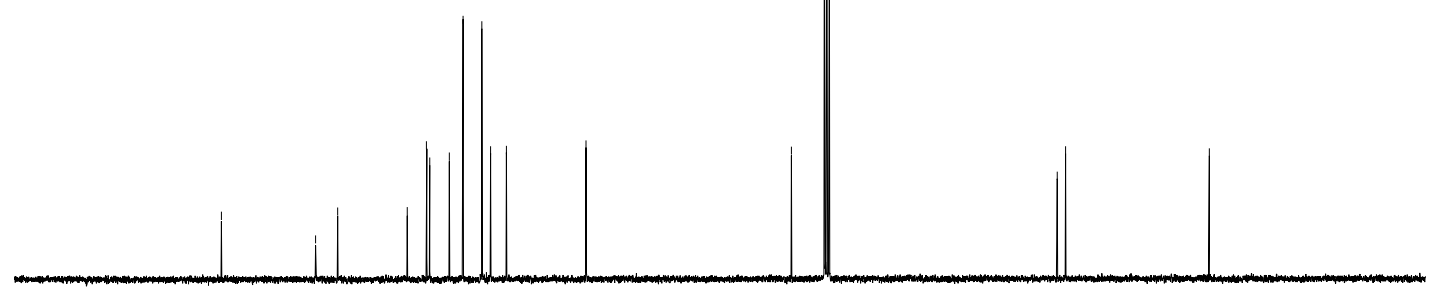


3ai

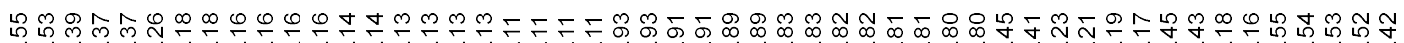

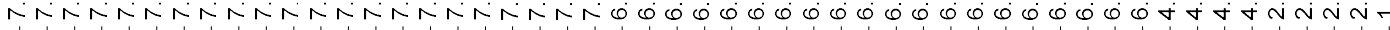
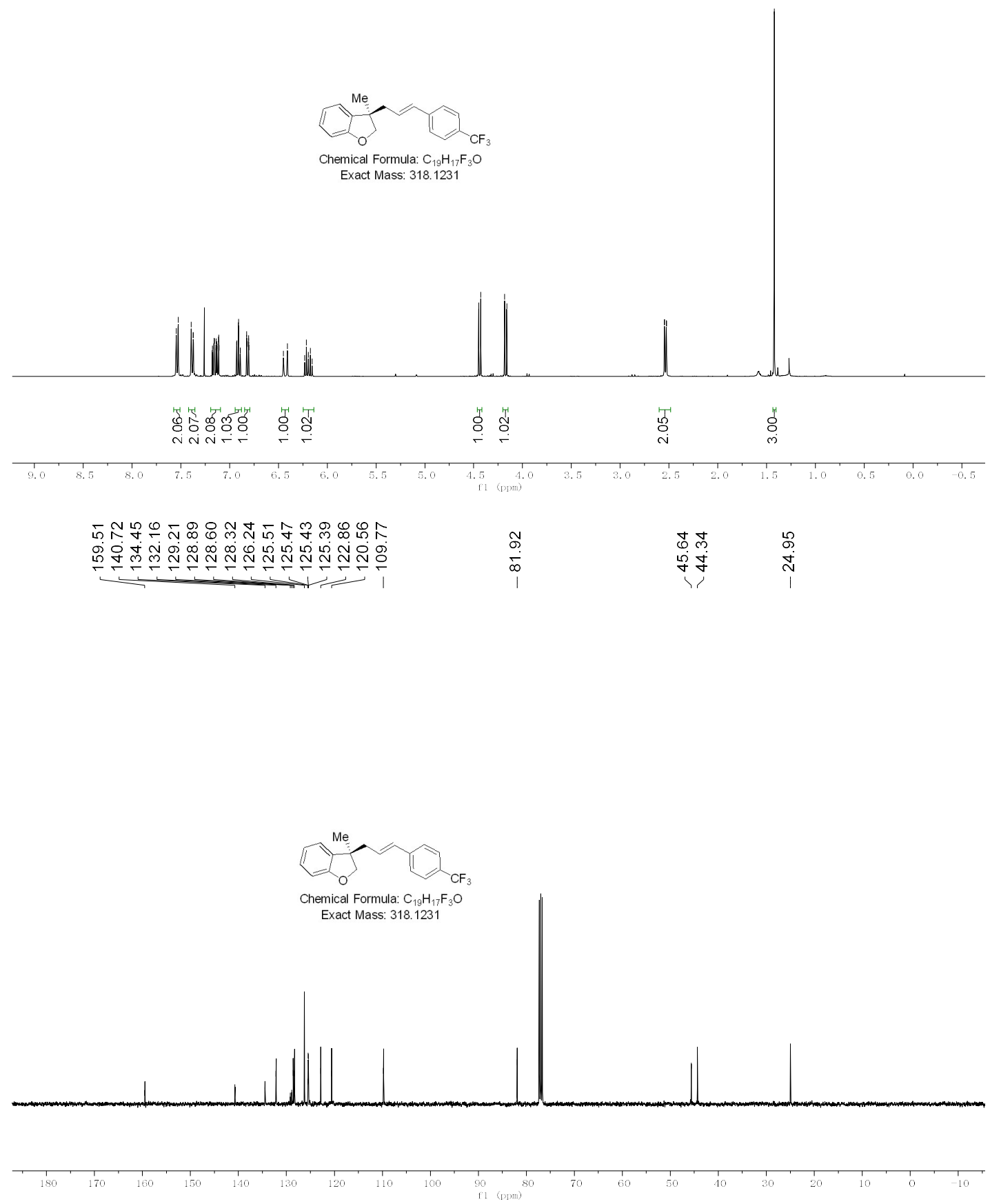


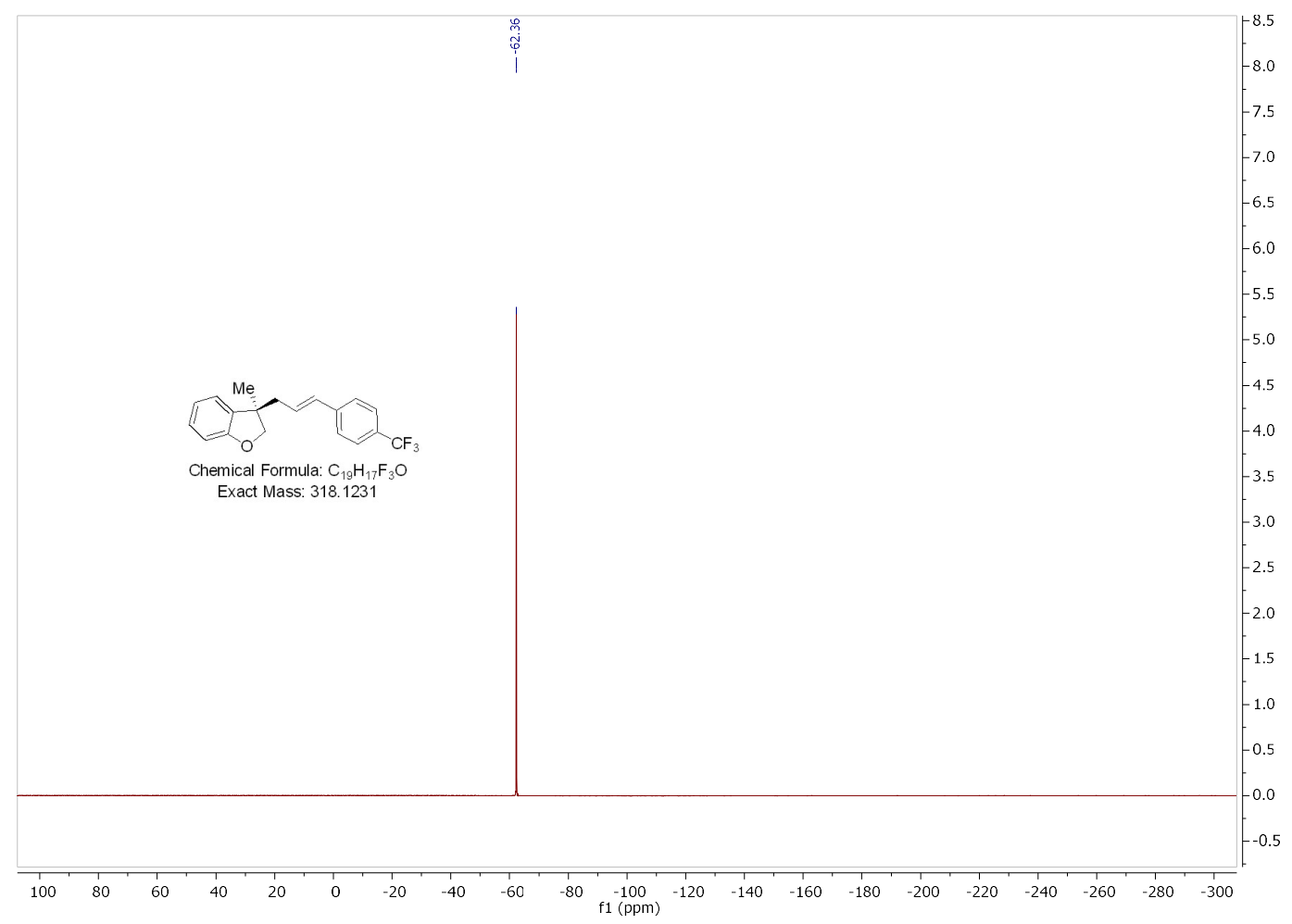


3aj

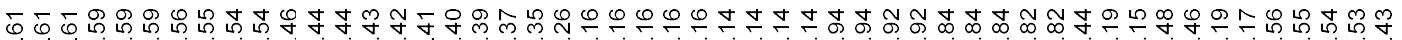

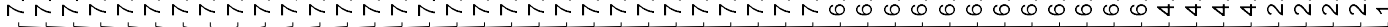

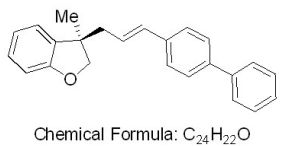

Exact Mass: 326.1671
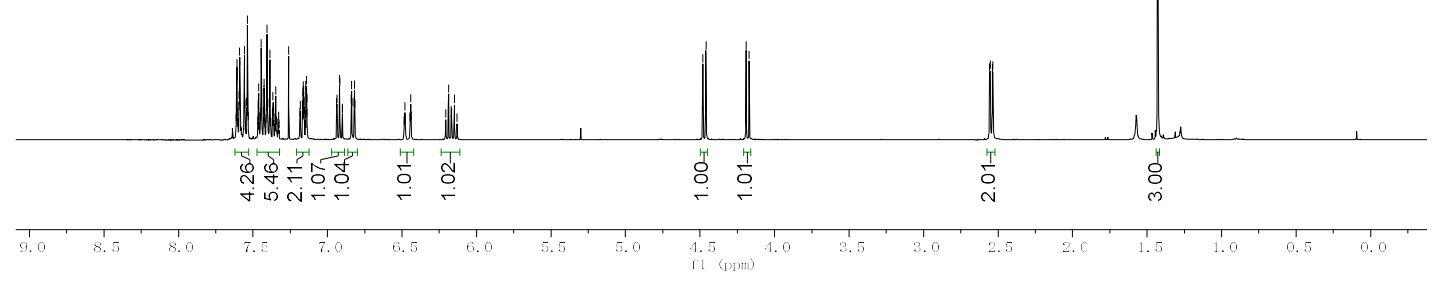

மำ

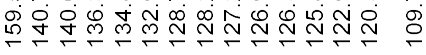

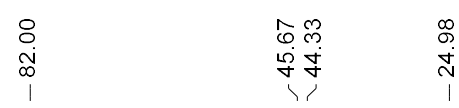

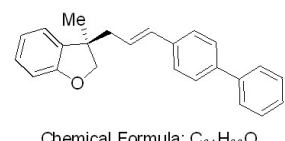

Chemical Formula: $\mathrm{C}_{24} \mathrm{H}_{22} \mathrm{O}$

Exact Mass: 326.1671
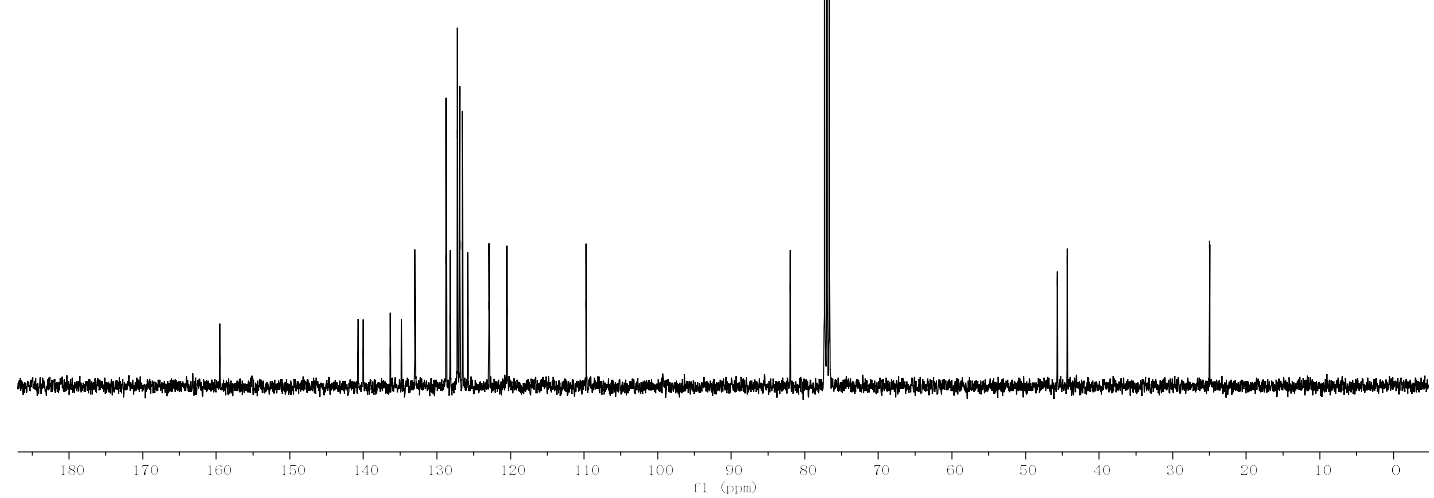
3ak

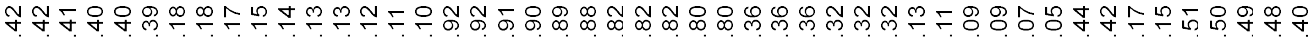

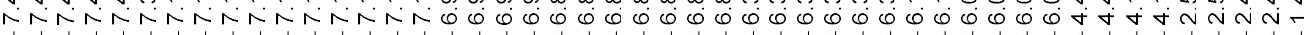
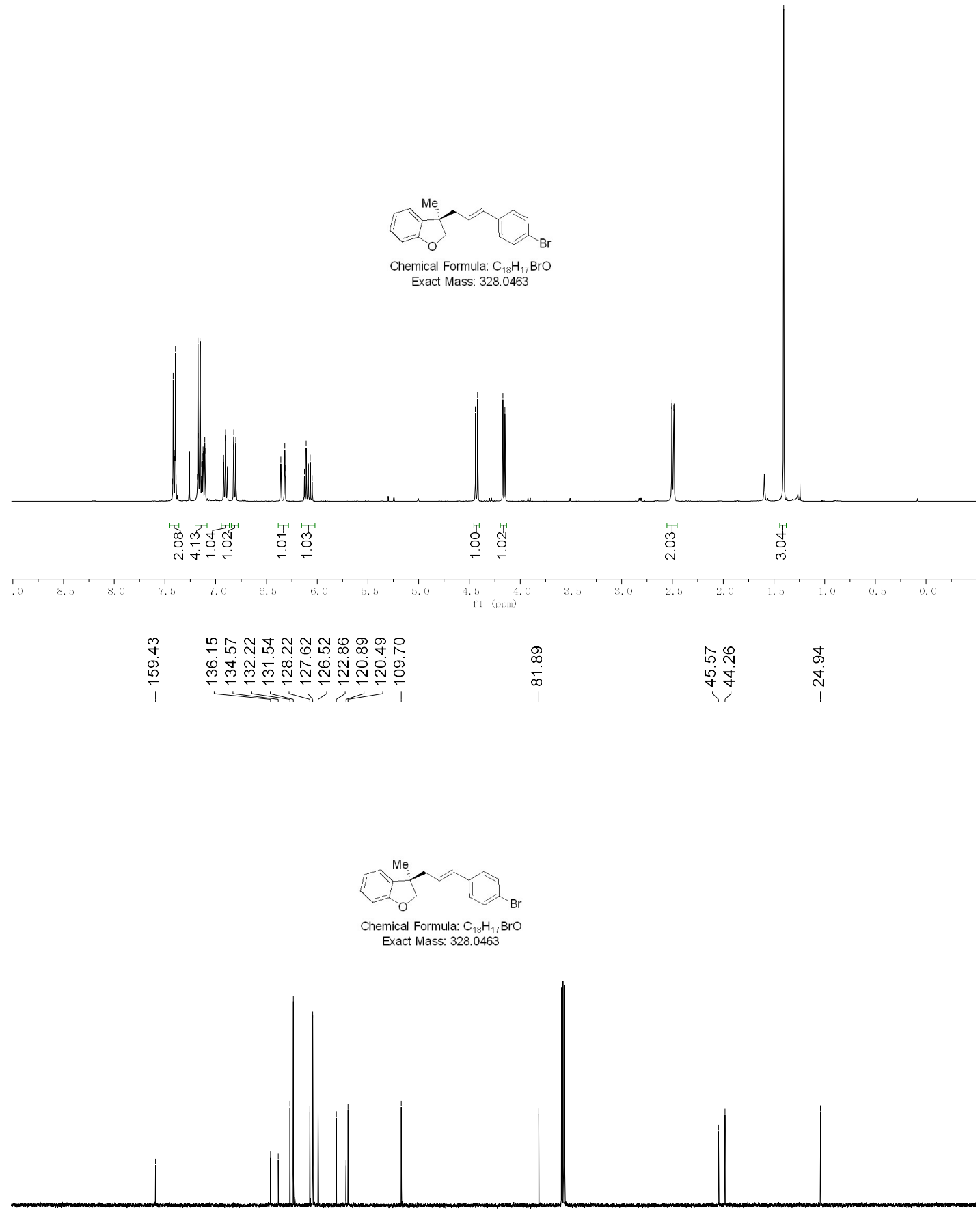
3al

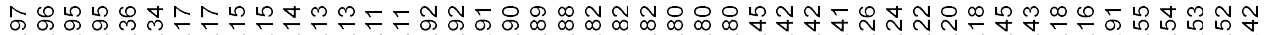

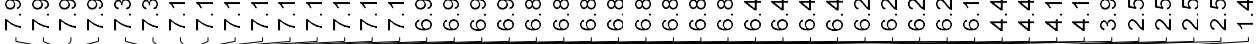
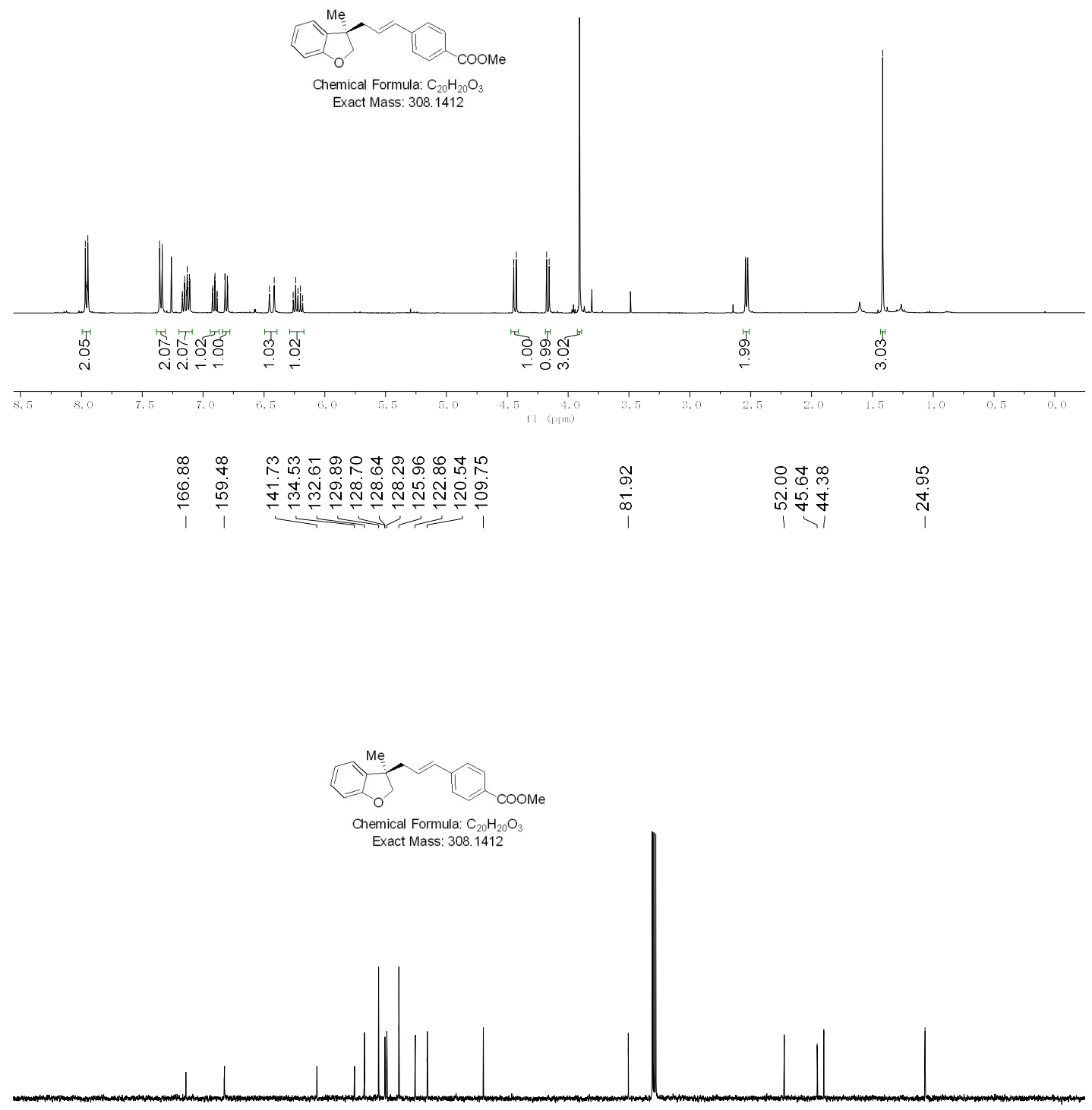

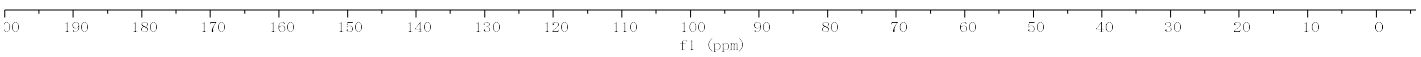


3am

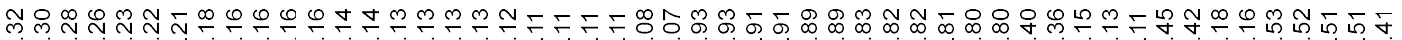

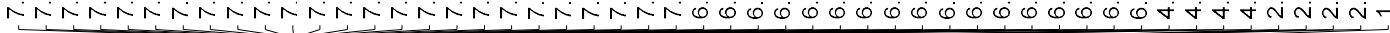
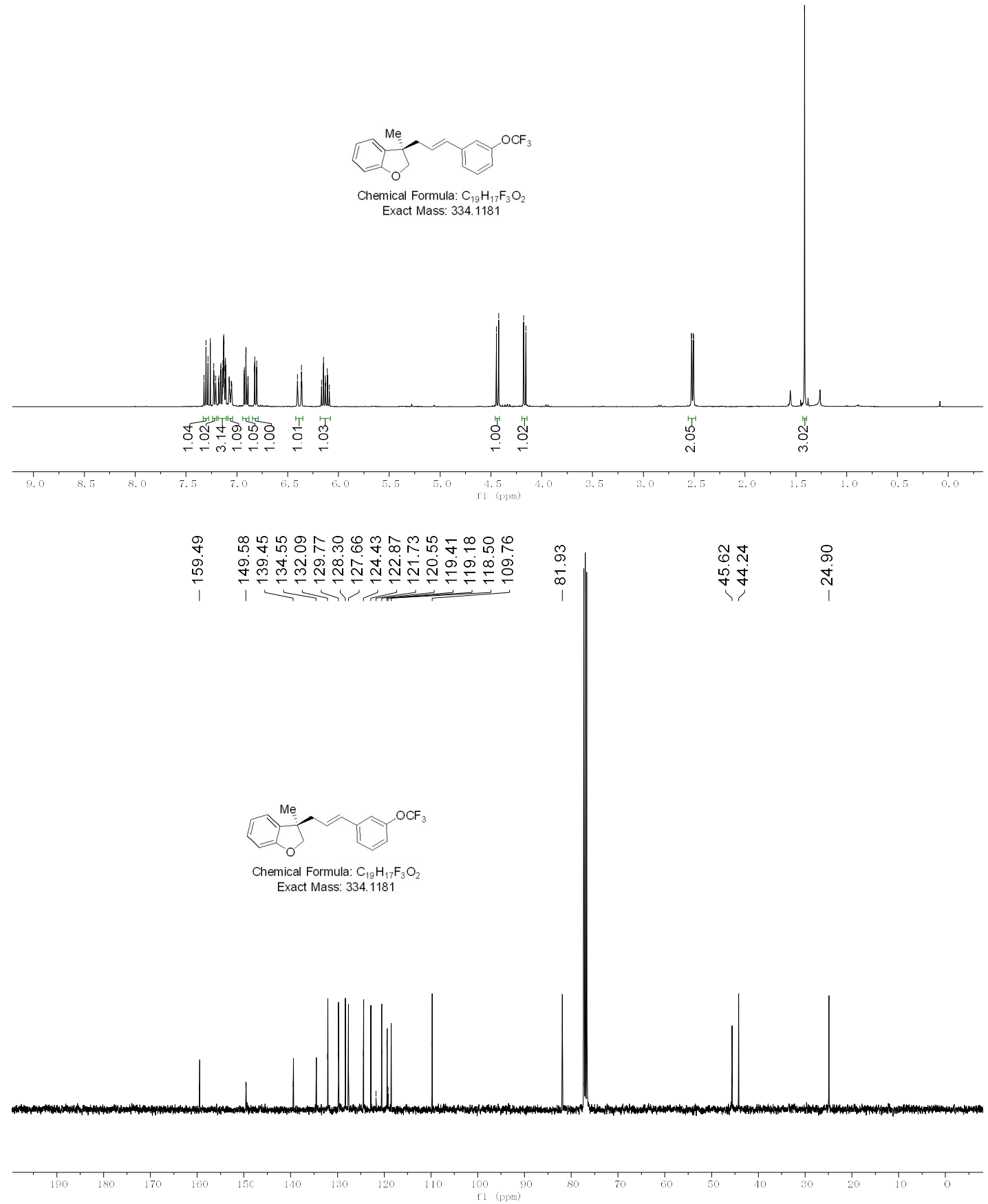


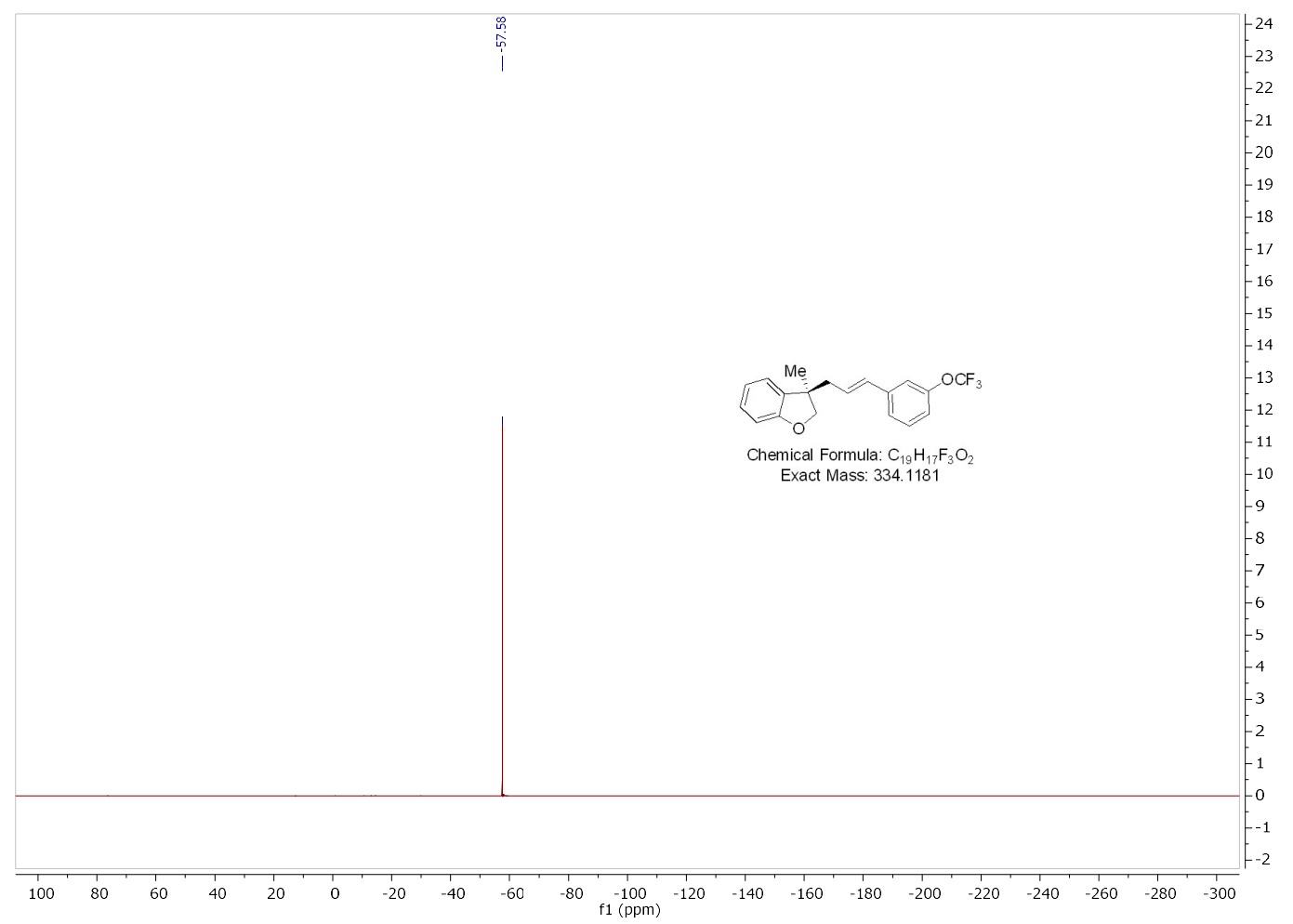


3an

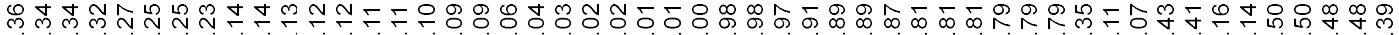

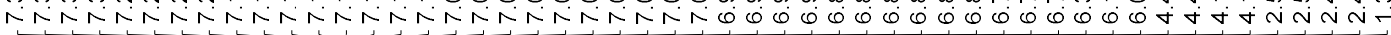
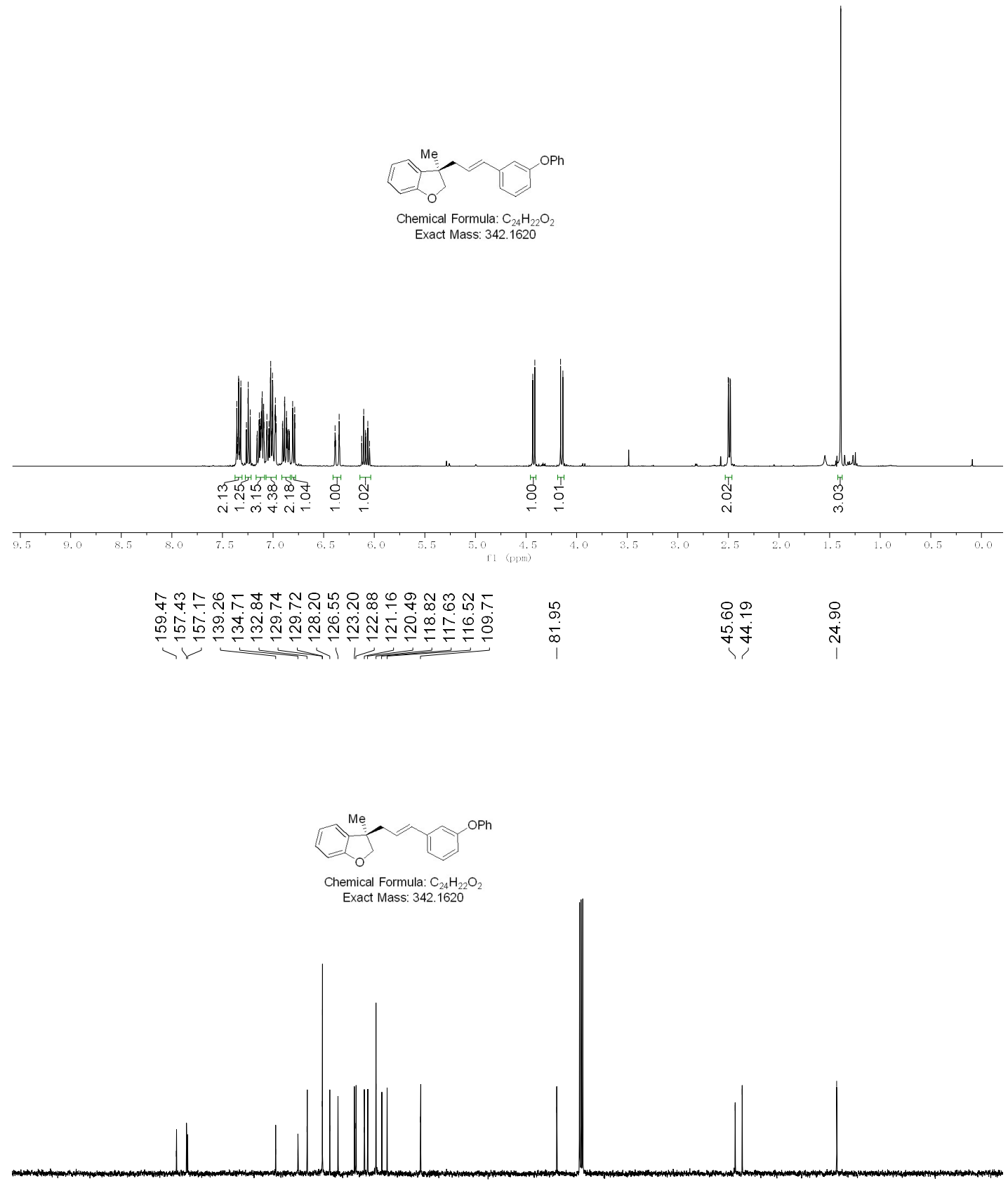
3 ao

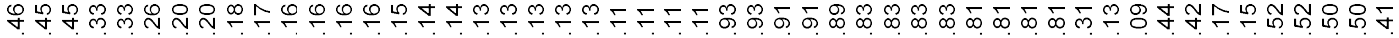

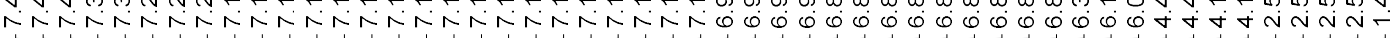

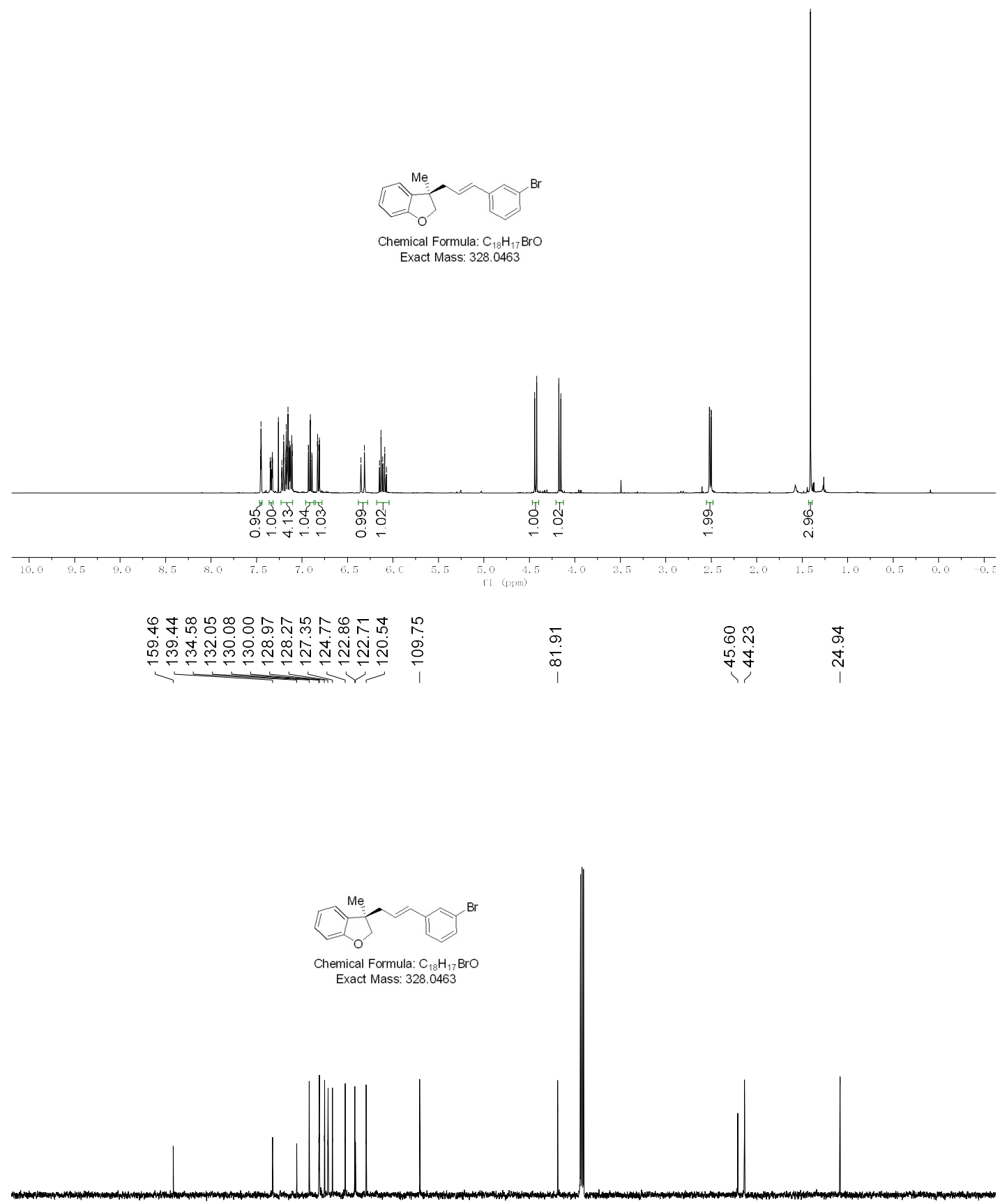

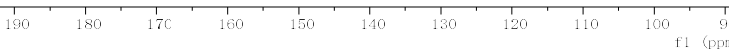


3ap

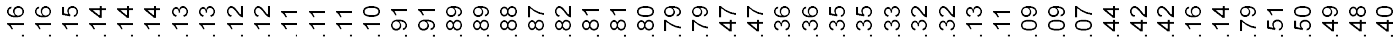

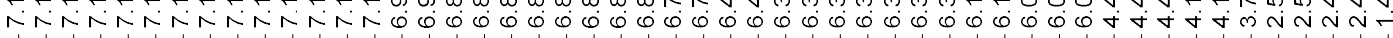
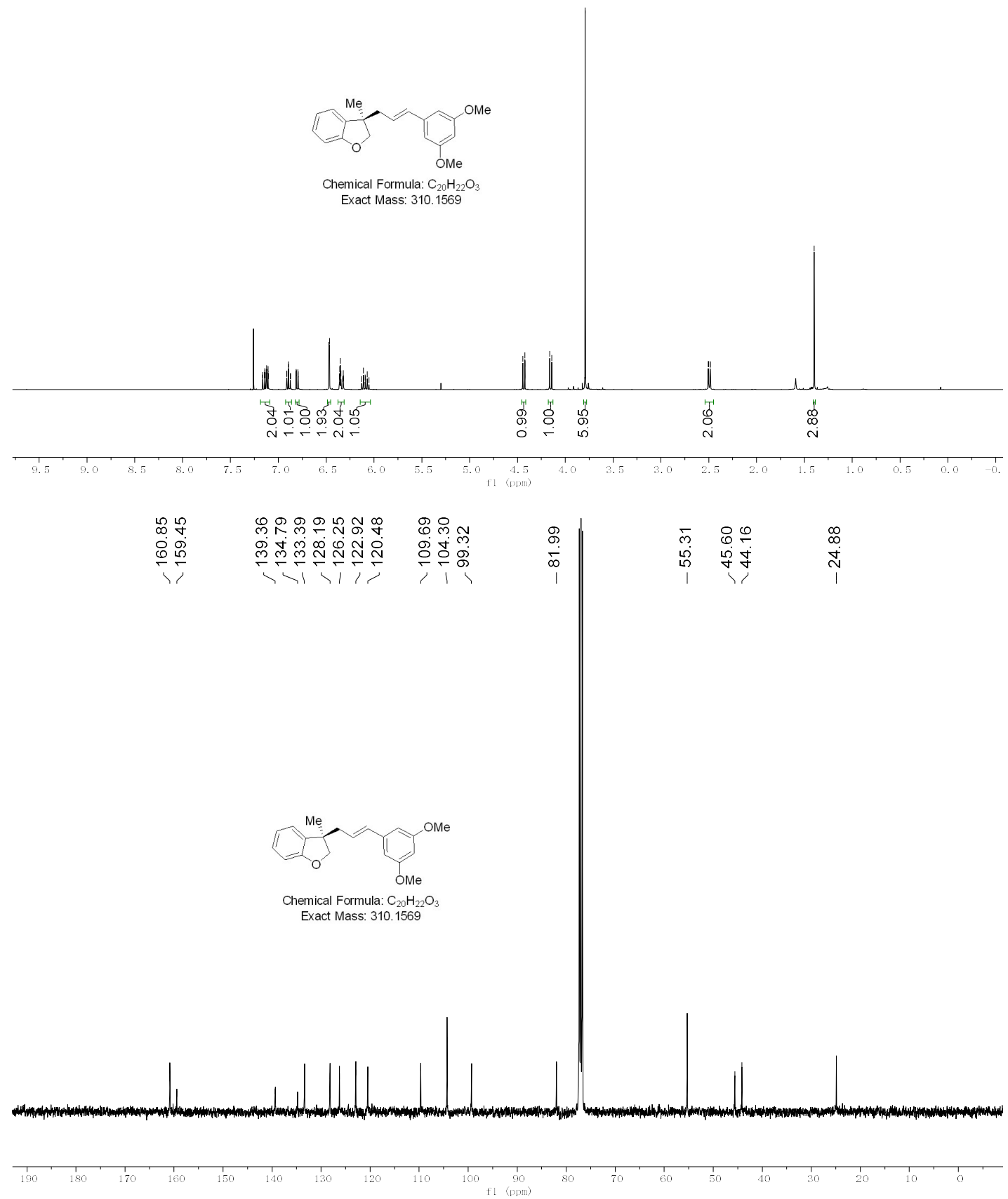
$3 a q$

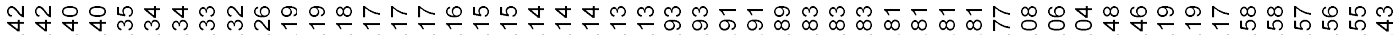

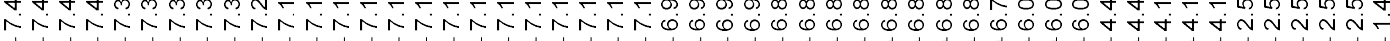
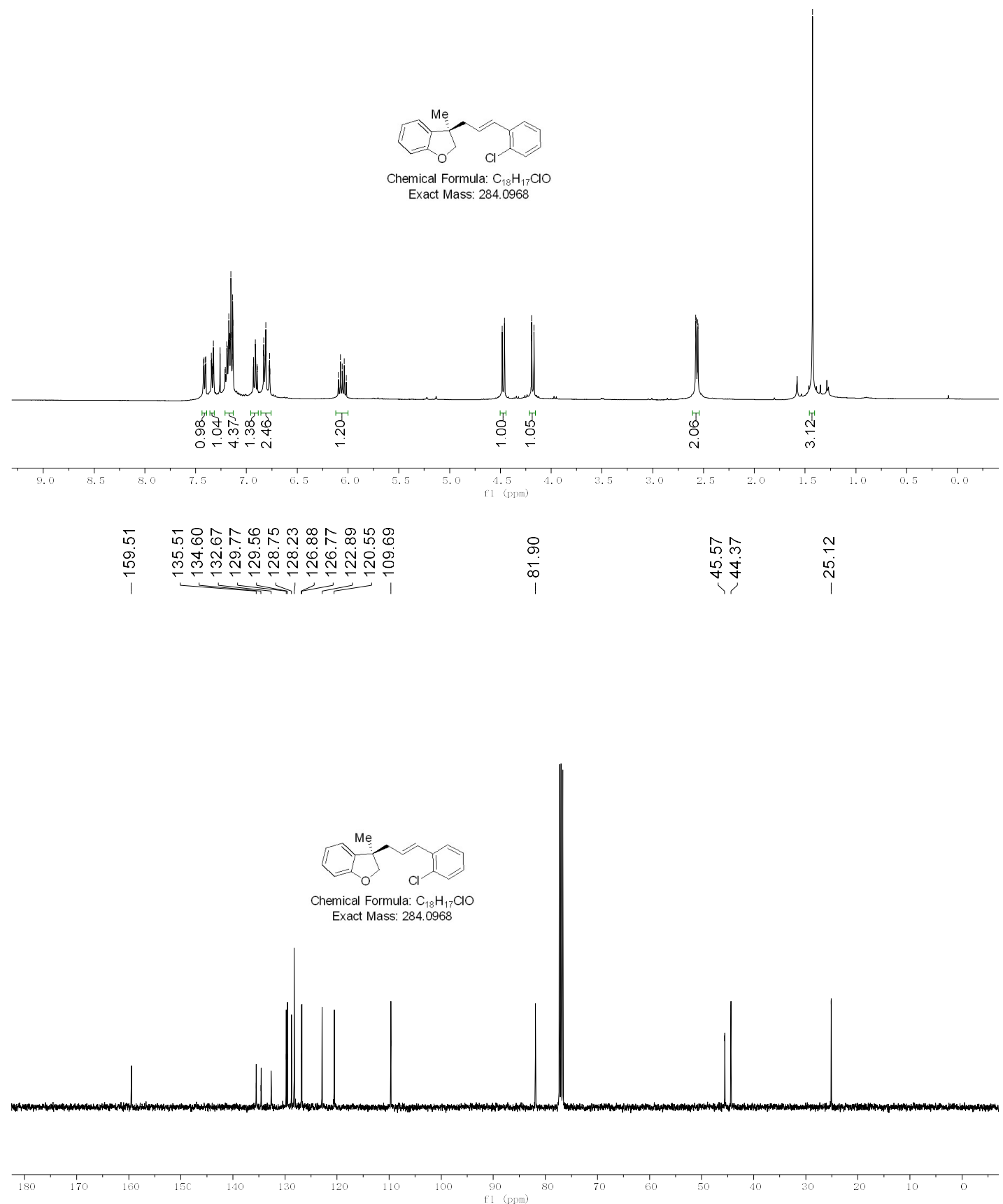
3ar

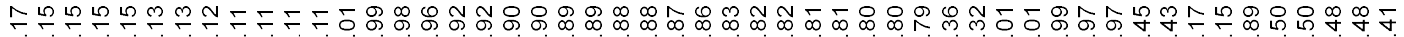

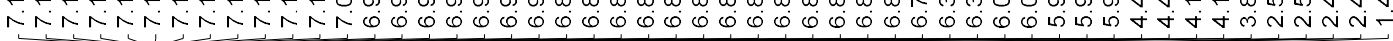
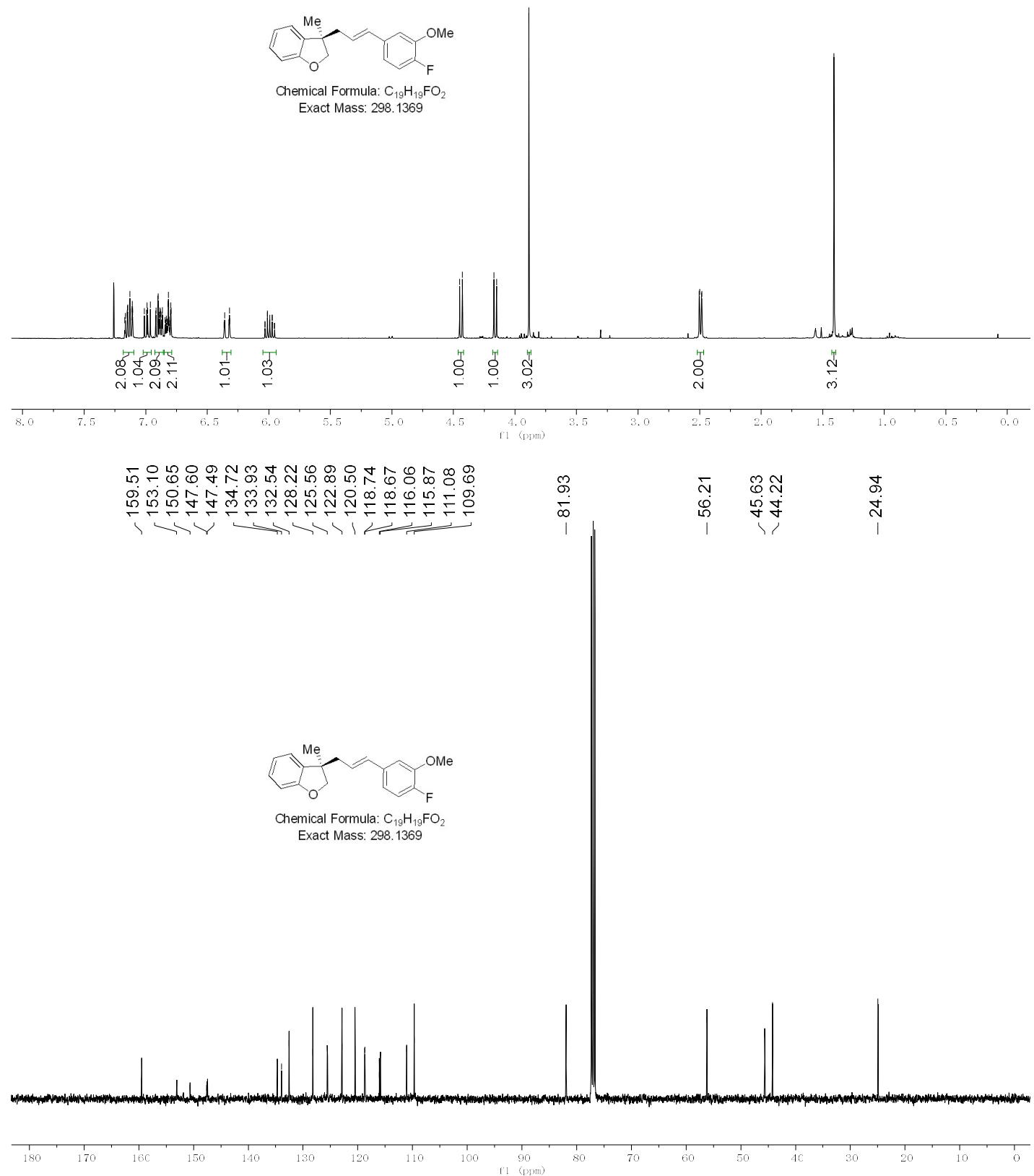


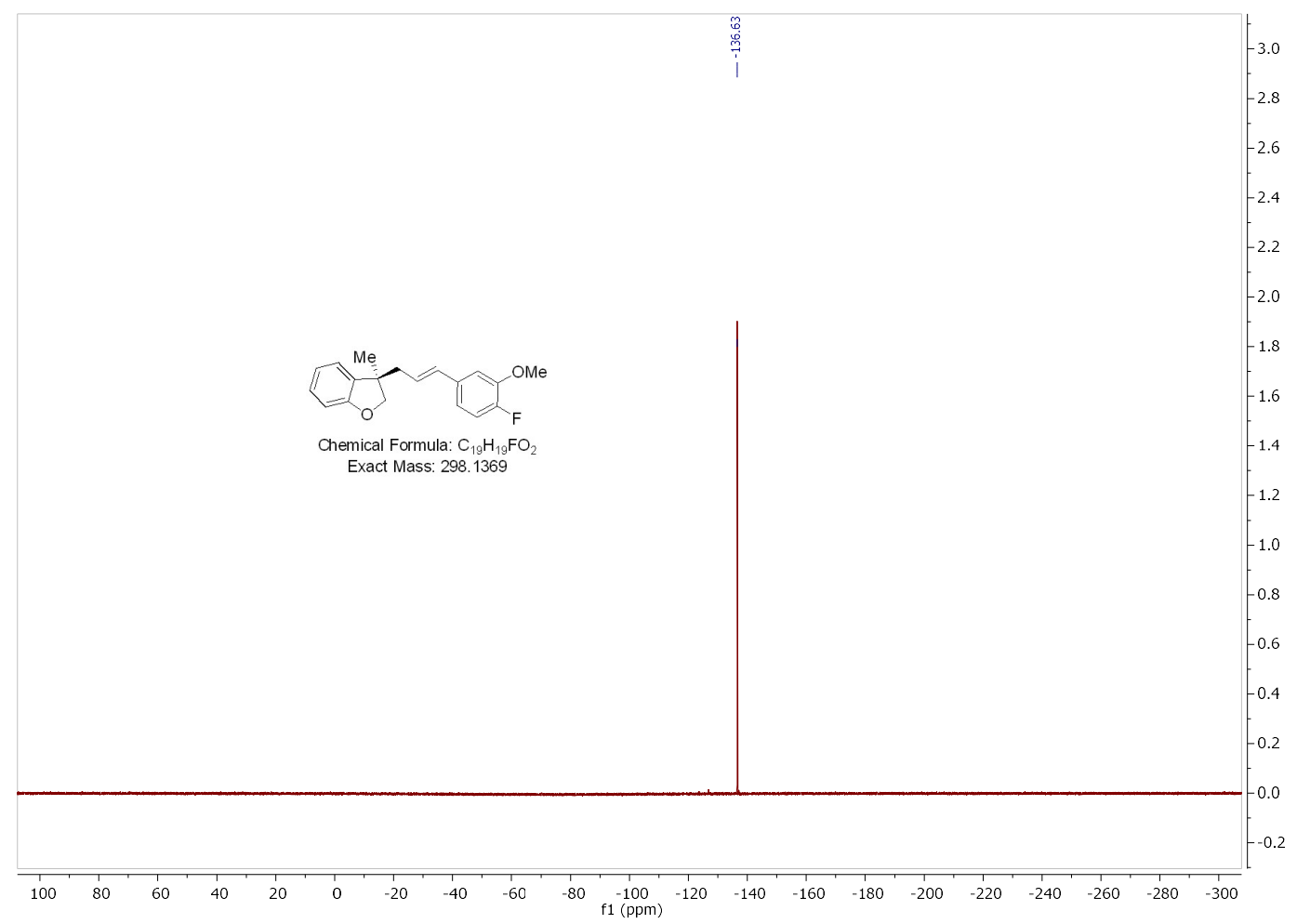


3as

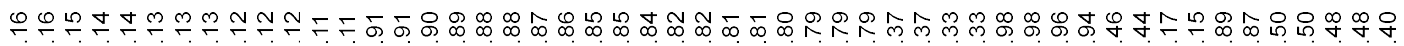

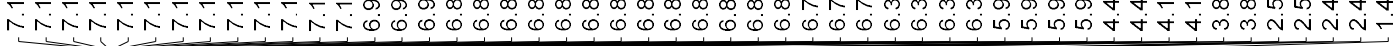
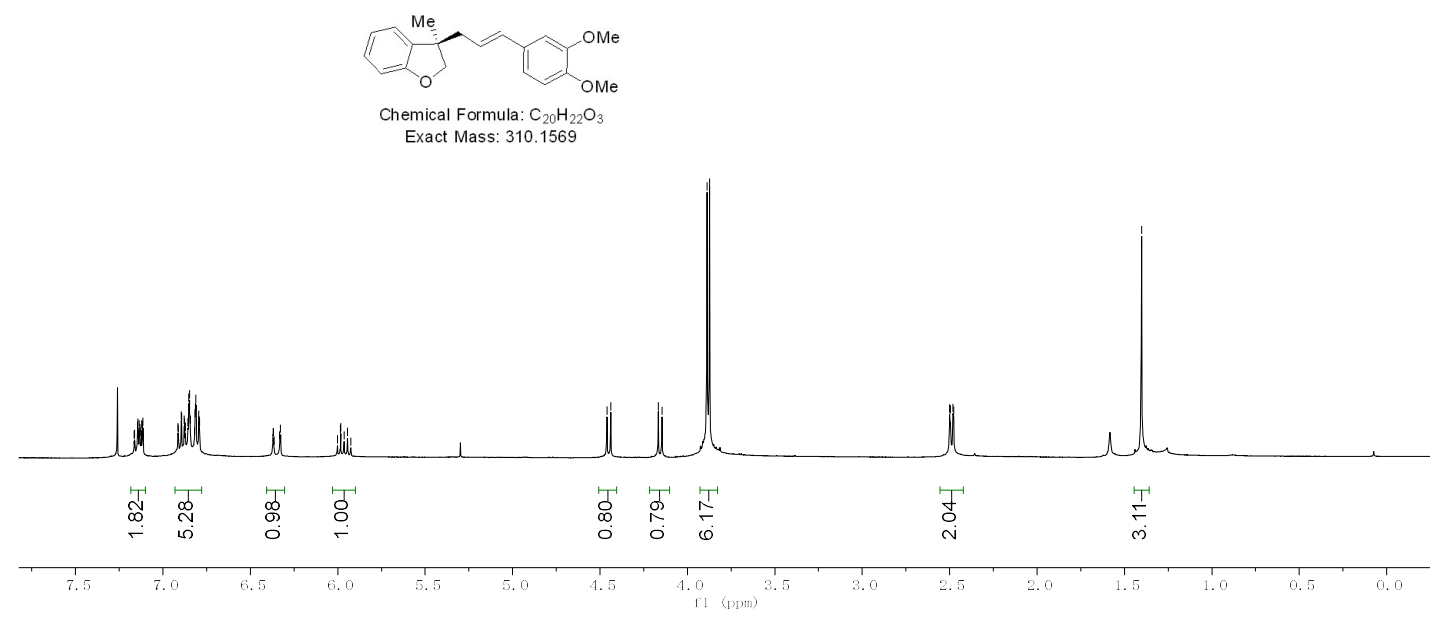

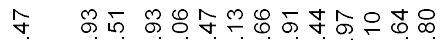

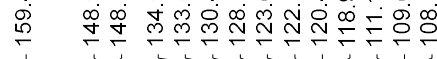

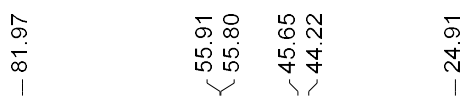

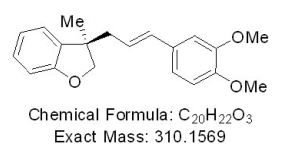

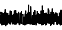

Exact Mass: 310.1569
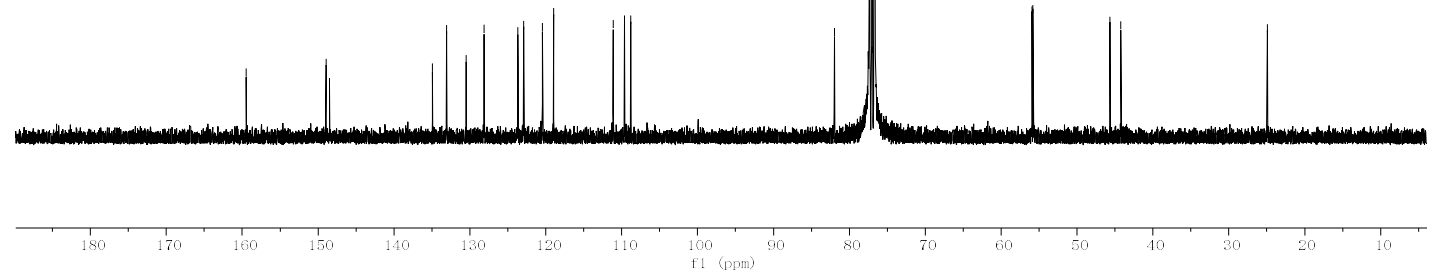
3at

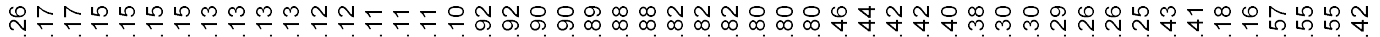

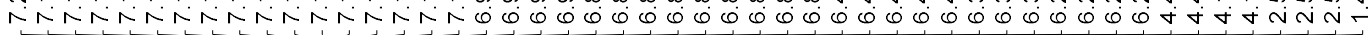
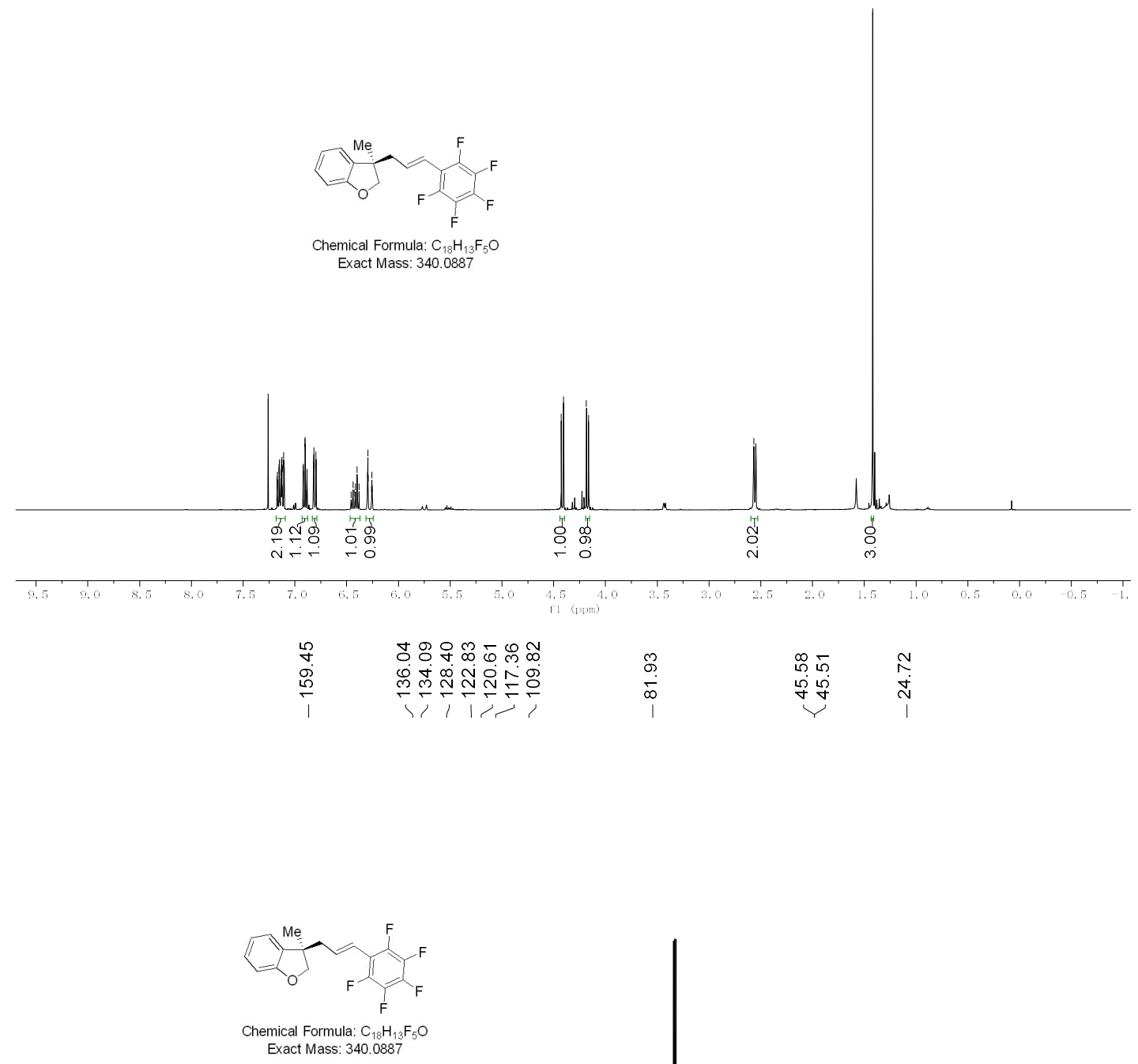

Exact Mass: 340.0887

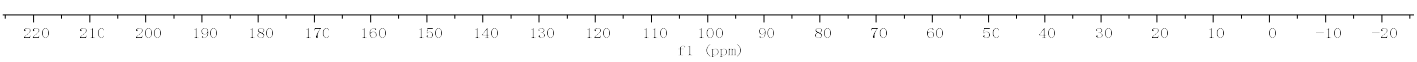




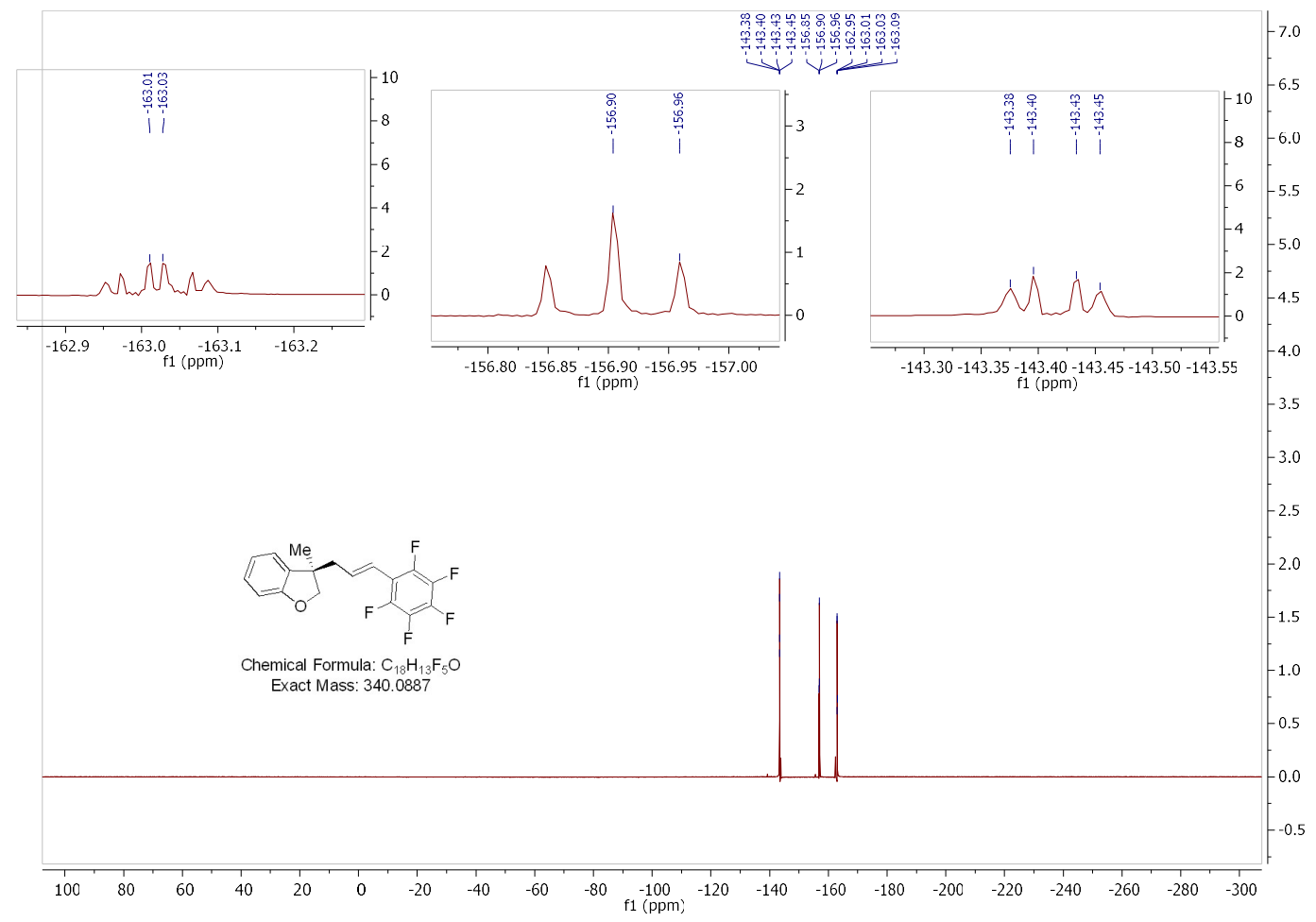


3bk

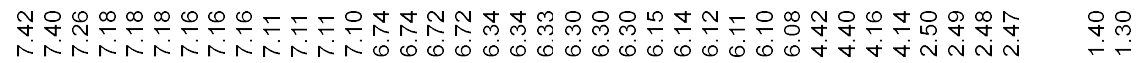

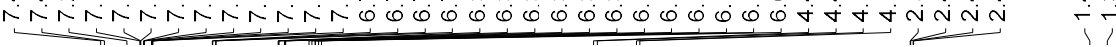
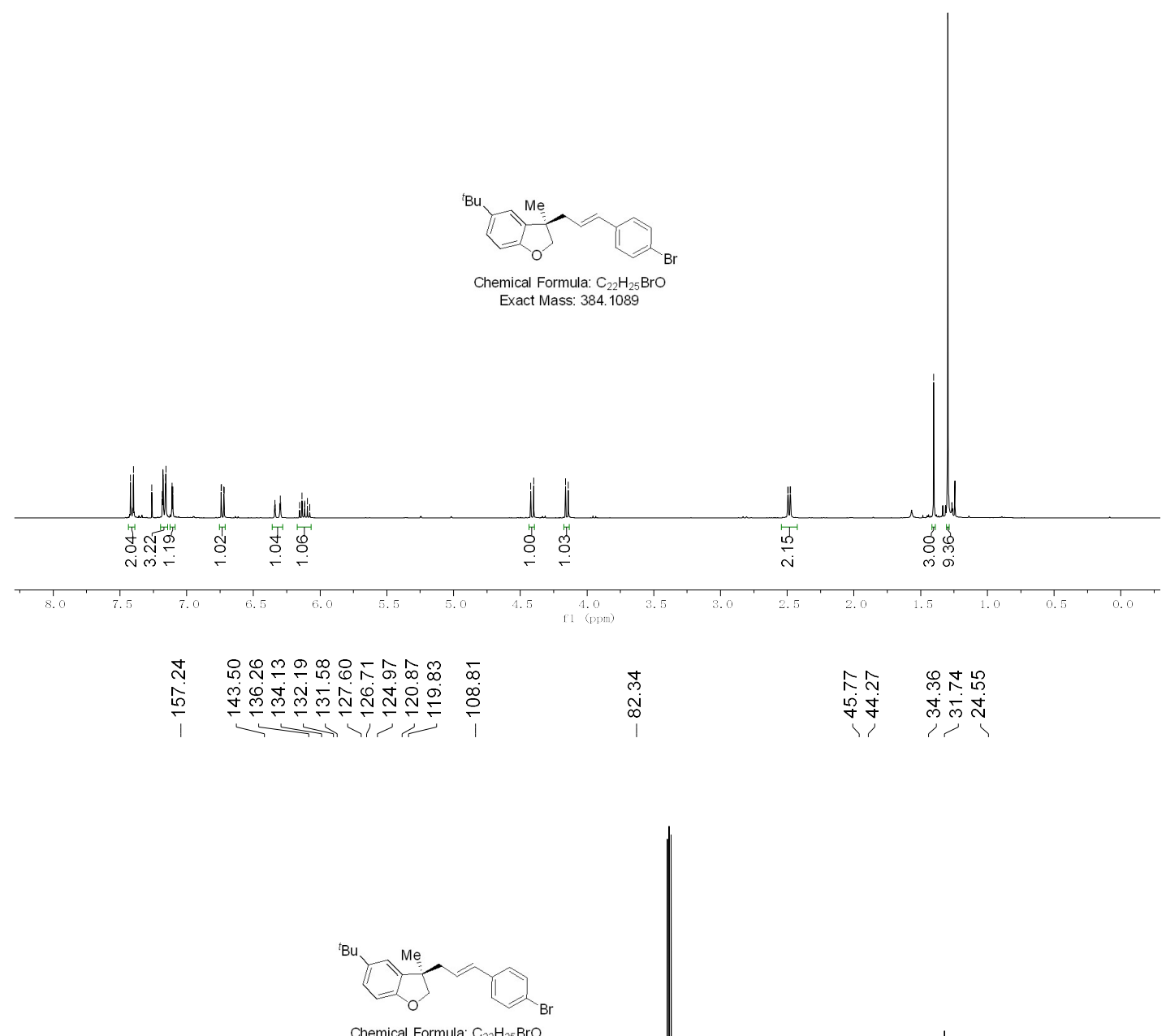

Chemical Formula: $\mathrm{C}_{22} \mathrm{H}_{25} \mathrm{BrO}$

Exact Mass: 384.1089

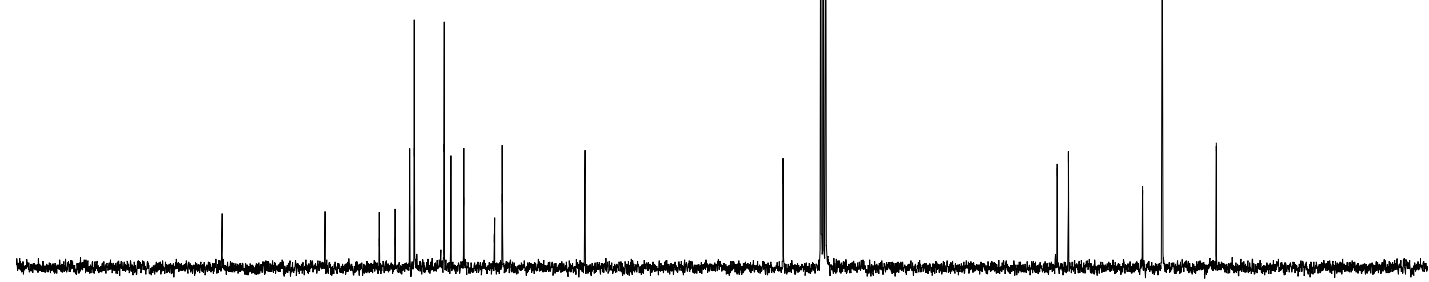




\section{3ck}

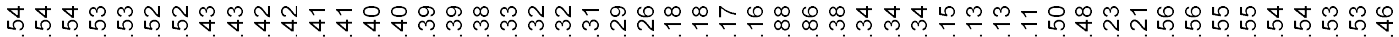

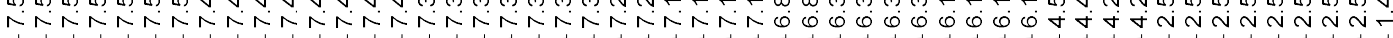
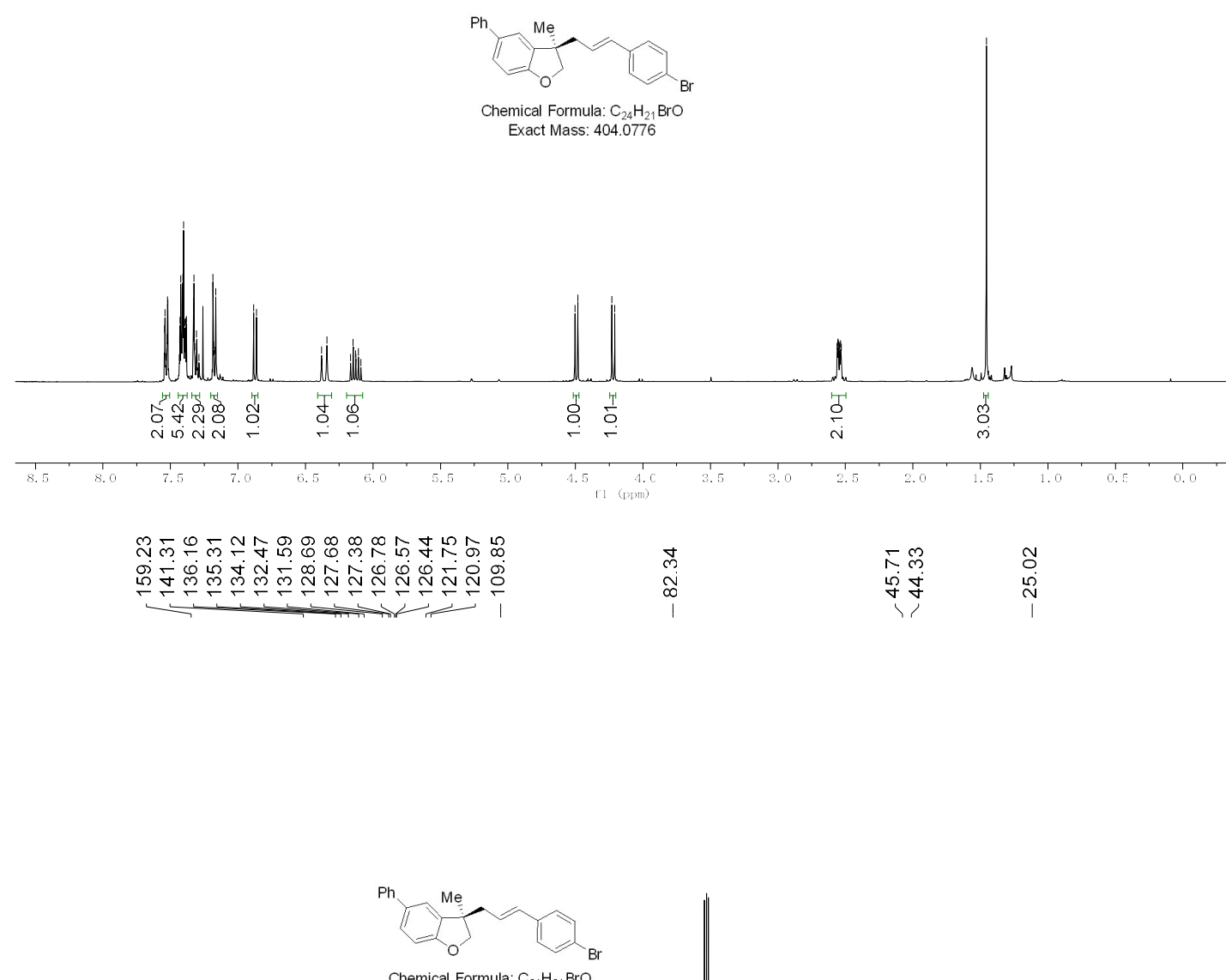

Chemical Formula: $\mathrm{C}_{24} \mathrm{H}_{21} \mathrm{BrO}$

Exact Mass: 404.0776
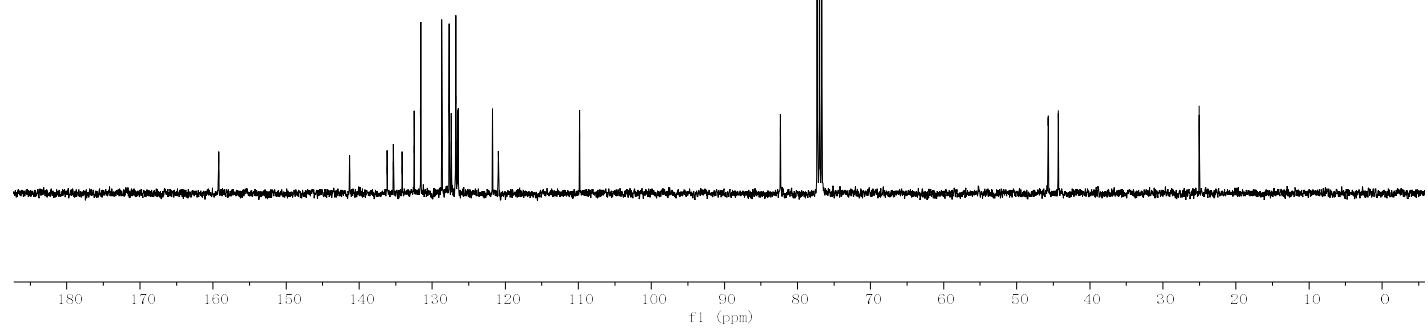
3dk

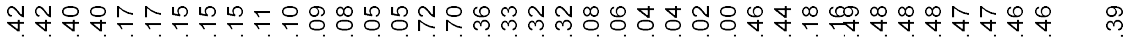

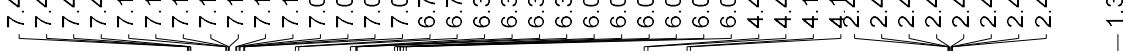
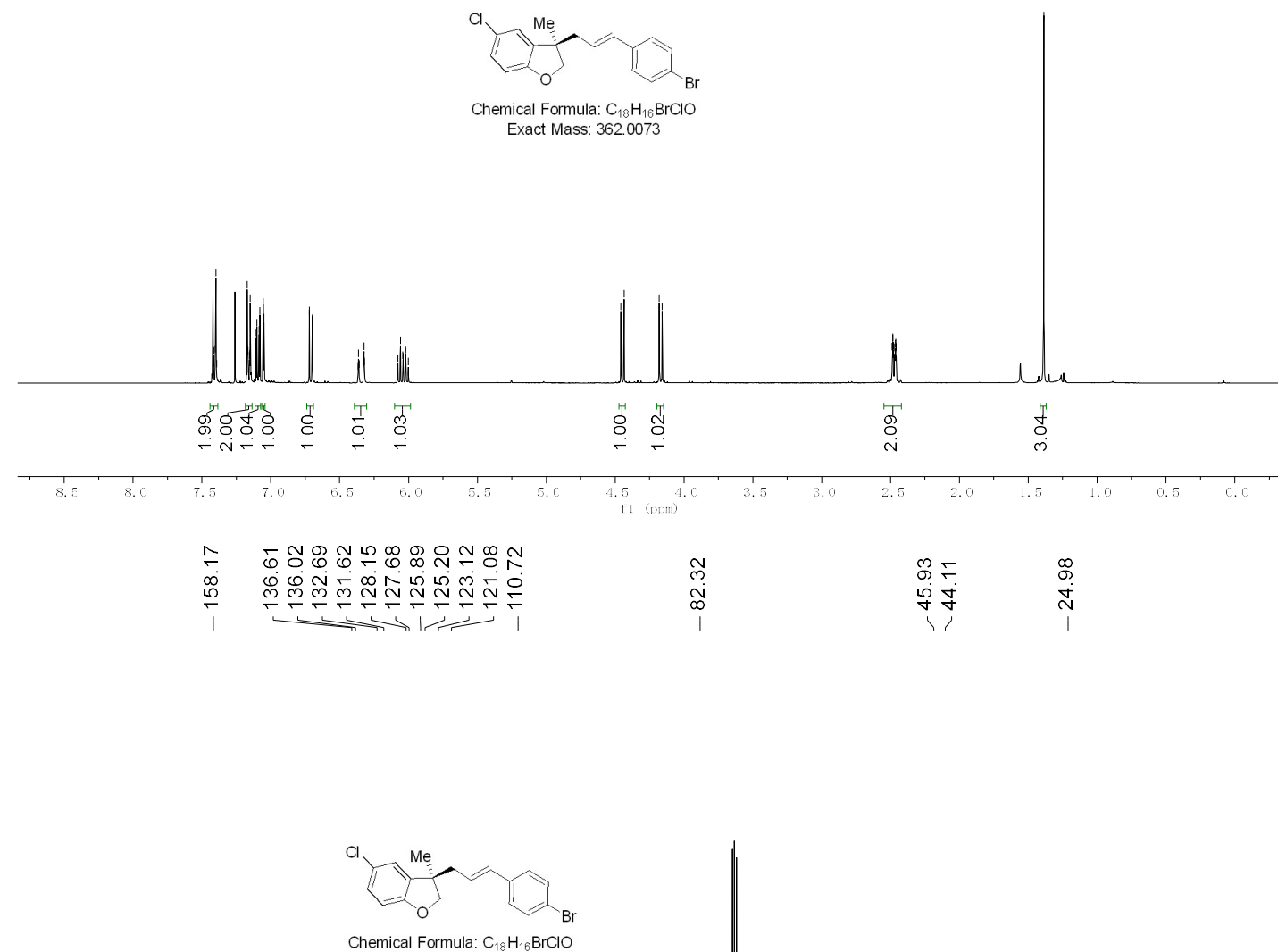

Exact Mass: 362.0073

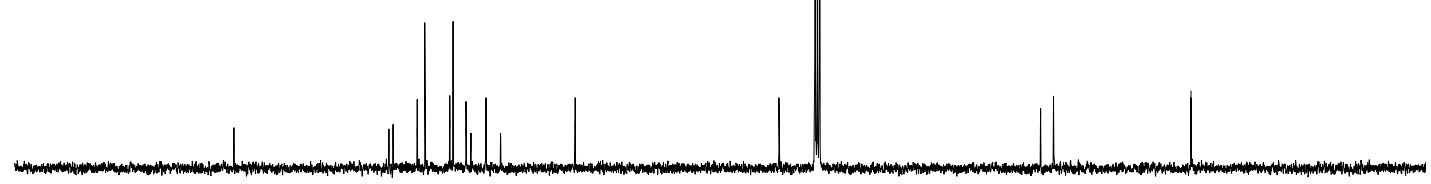




\section{3ek}

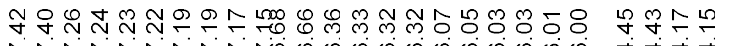

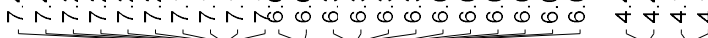

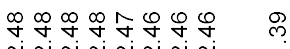

ningnivina

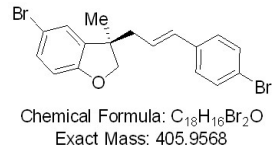

Exact Mass: 405.9568

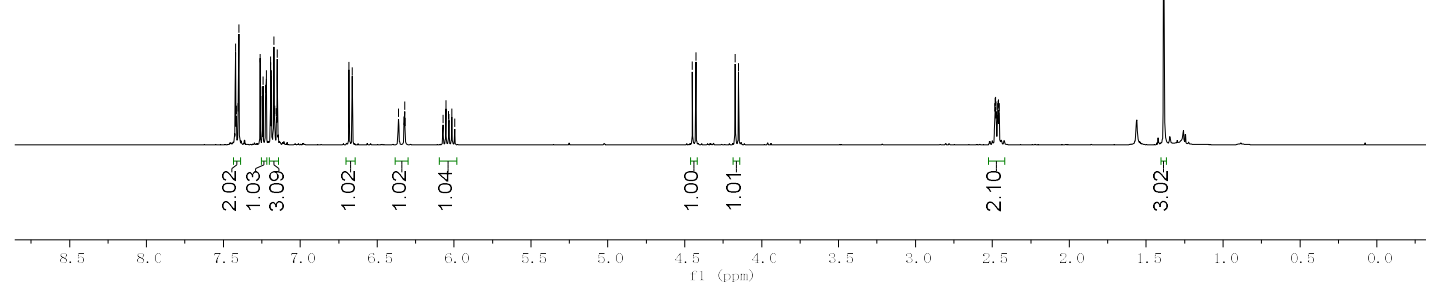

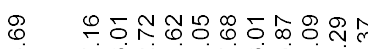

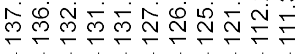

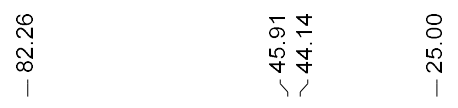
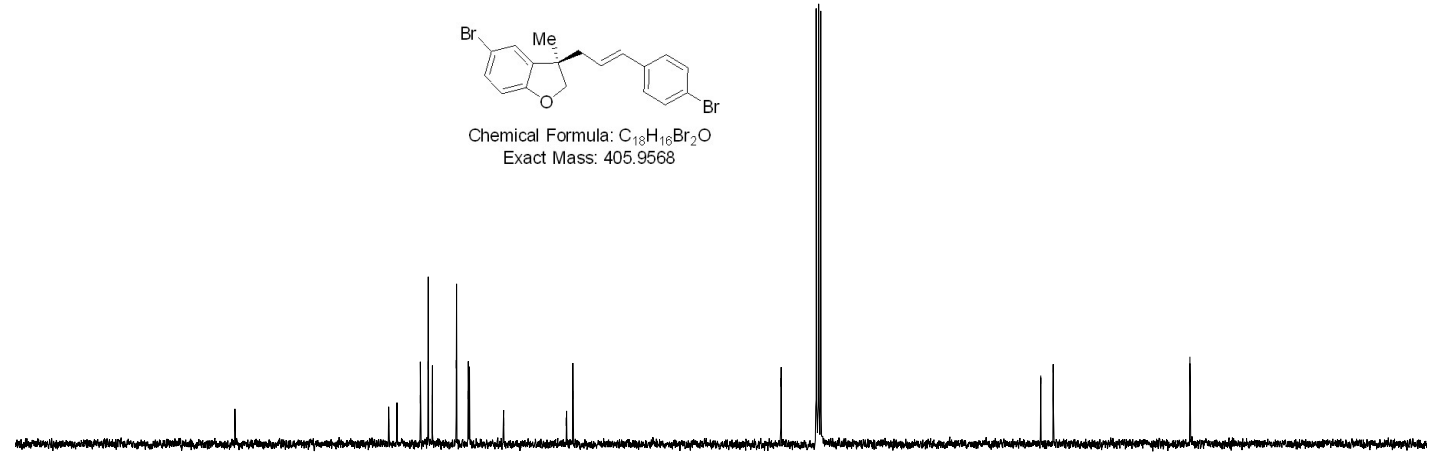
3fk

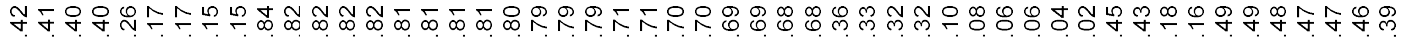

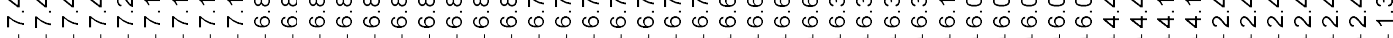
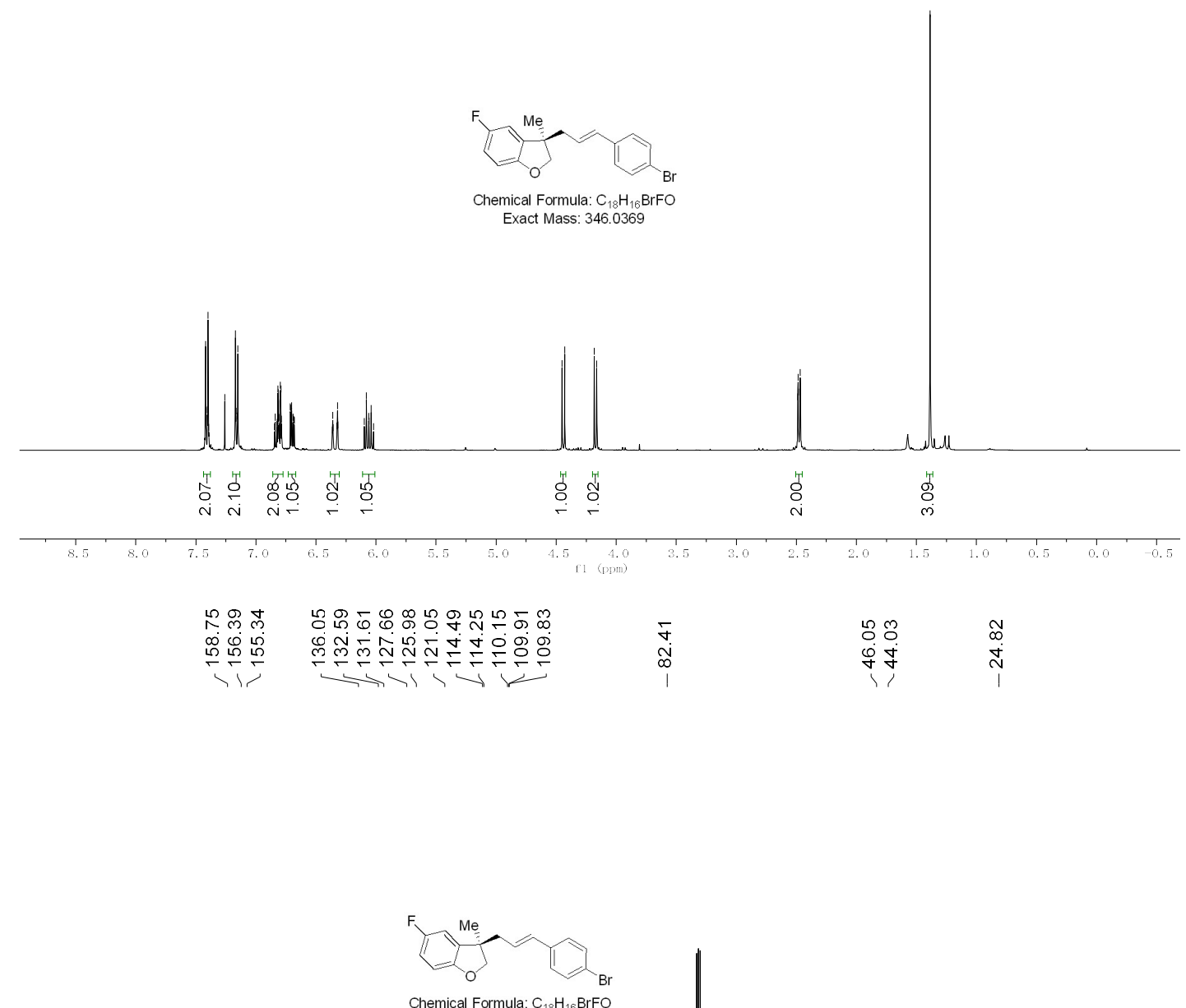

Exact Mass: 346.0369
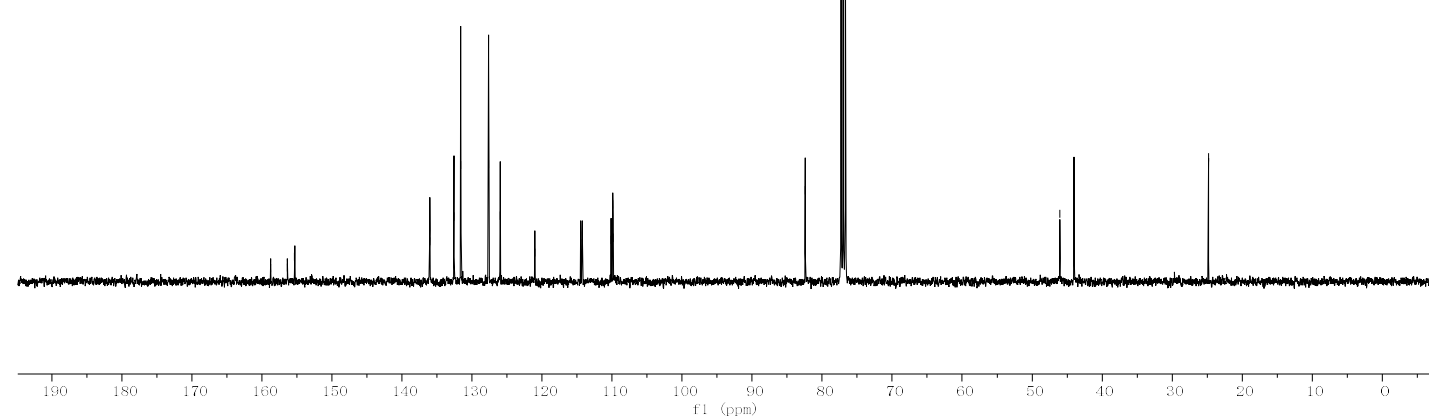
Chemical Formula: $\mathrm{C}_{18} \mathrm{H}_{16} \mathrm{BrFO}$

Exact Mass: 346.0369

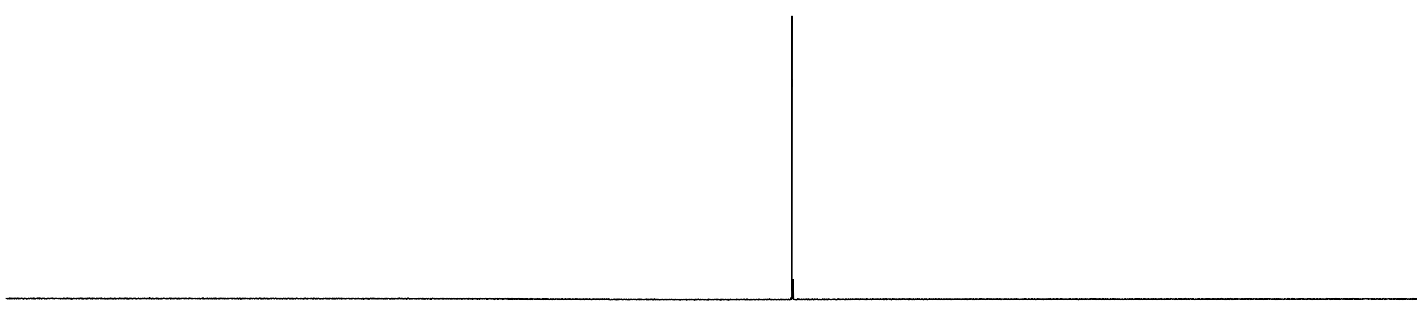

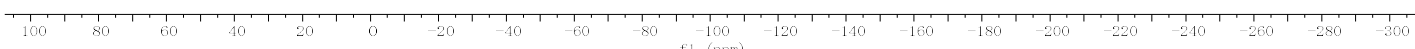

f1 (bom) 


\section{3gk}

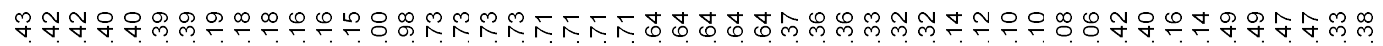

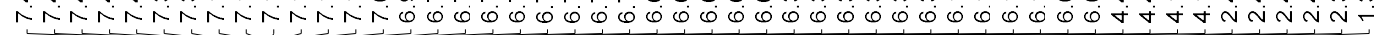
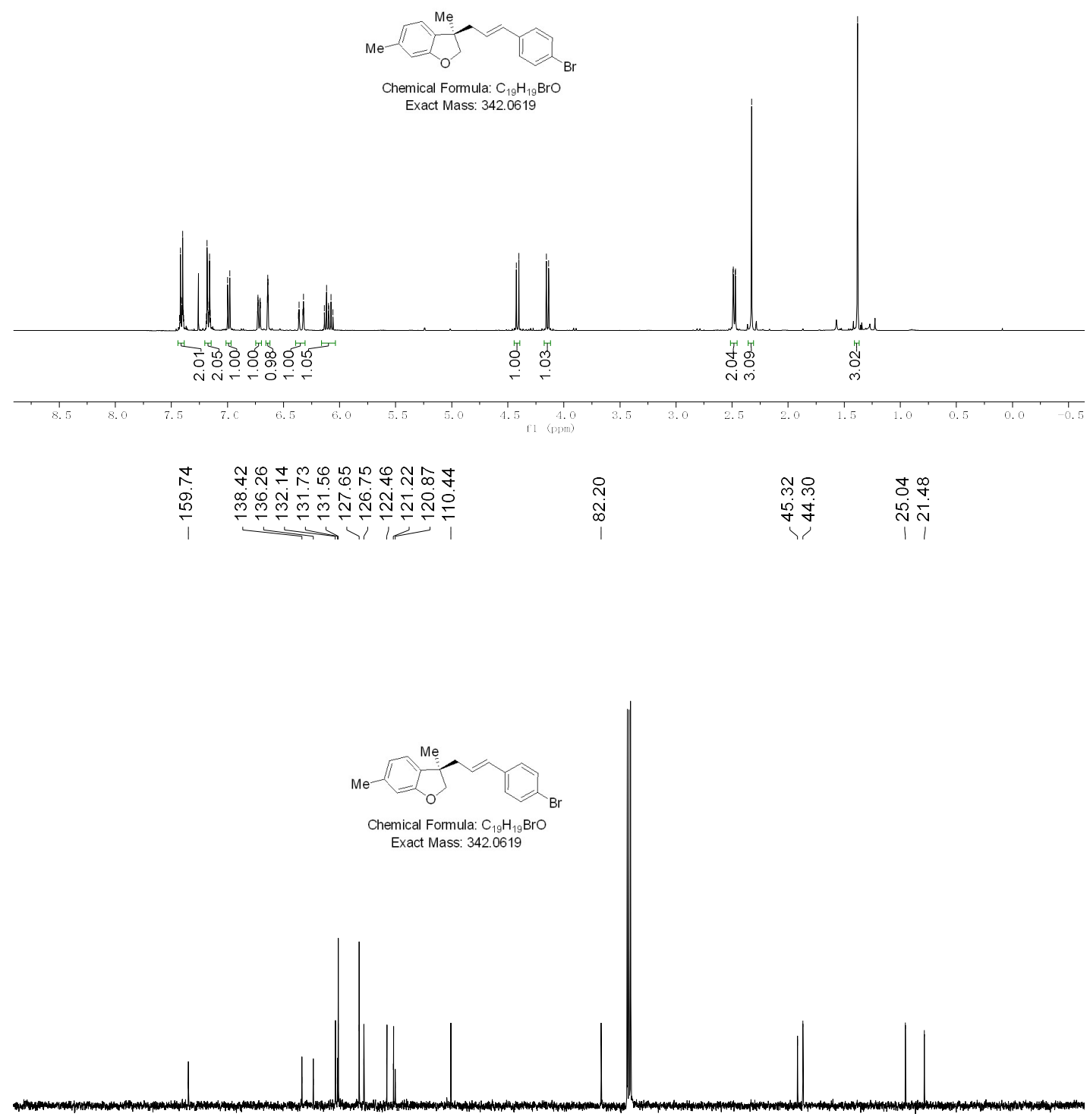

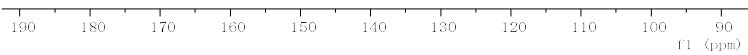




\section{3hk}

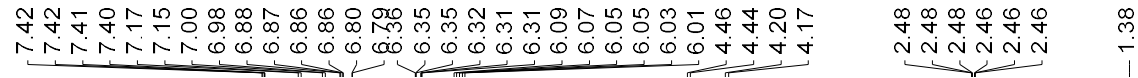
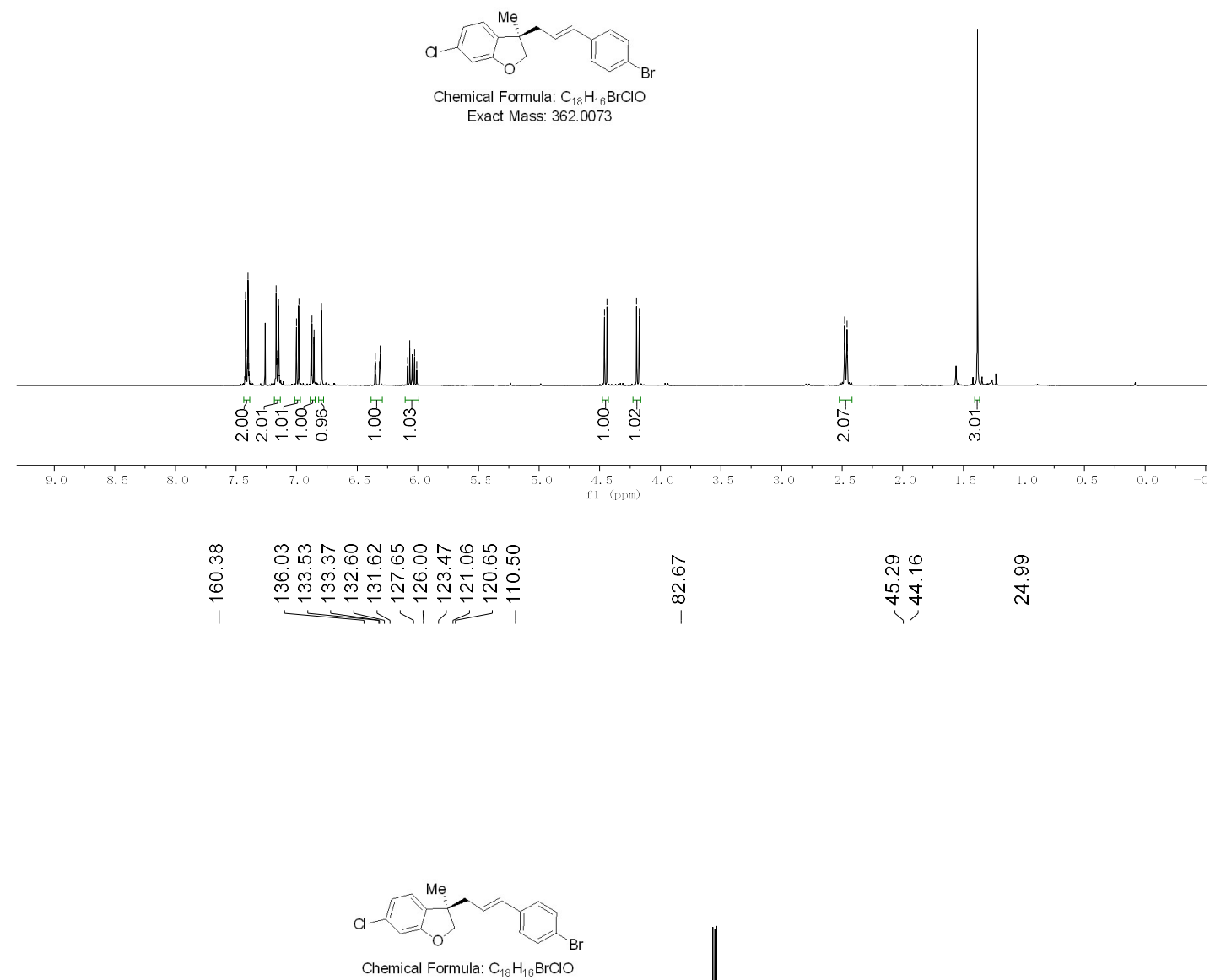
Exact Mass: 362.0073
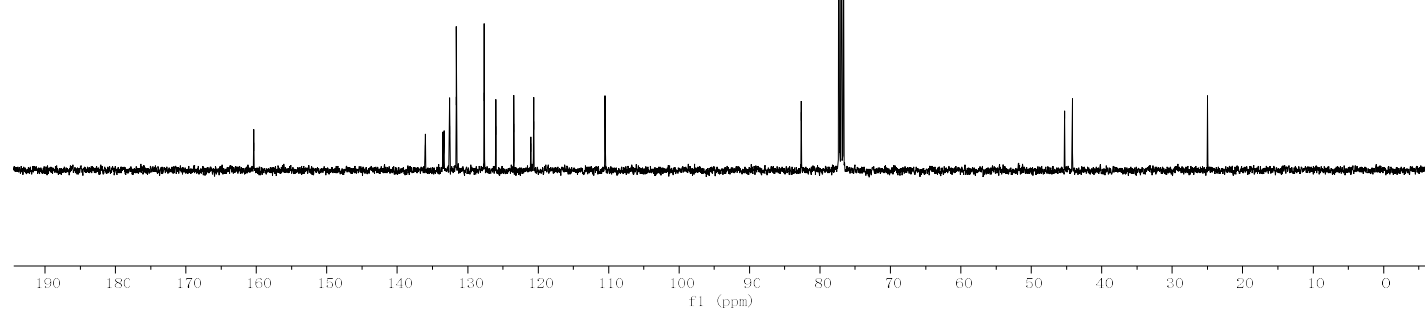


\section{4}

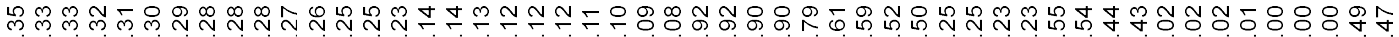

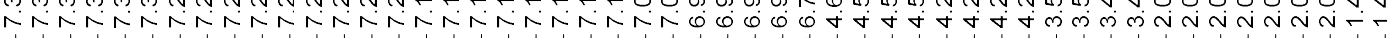
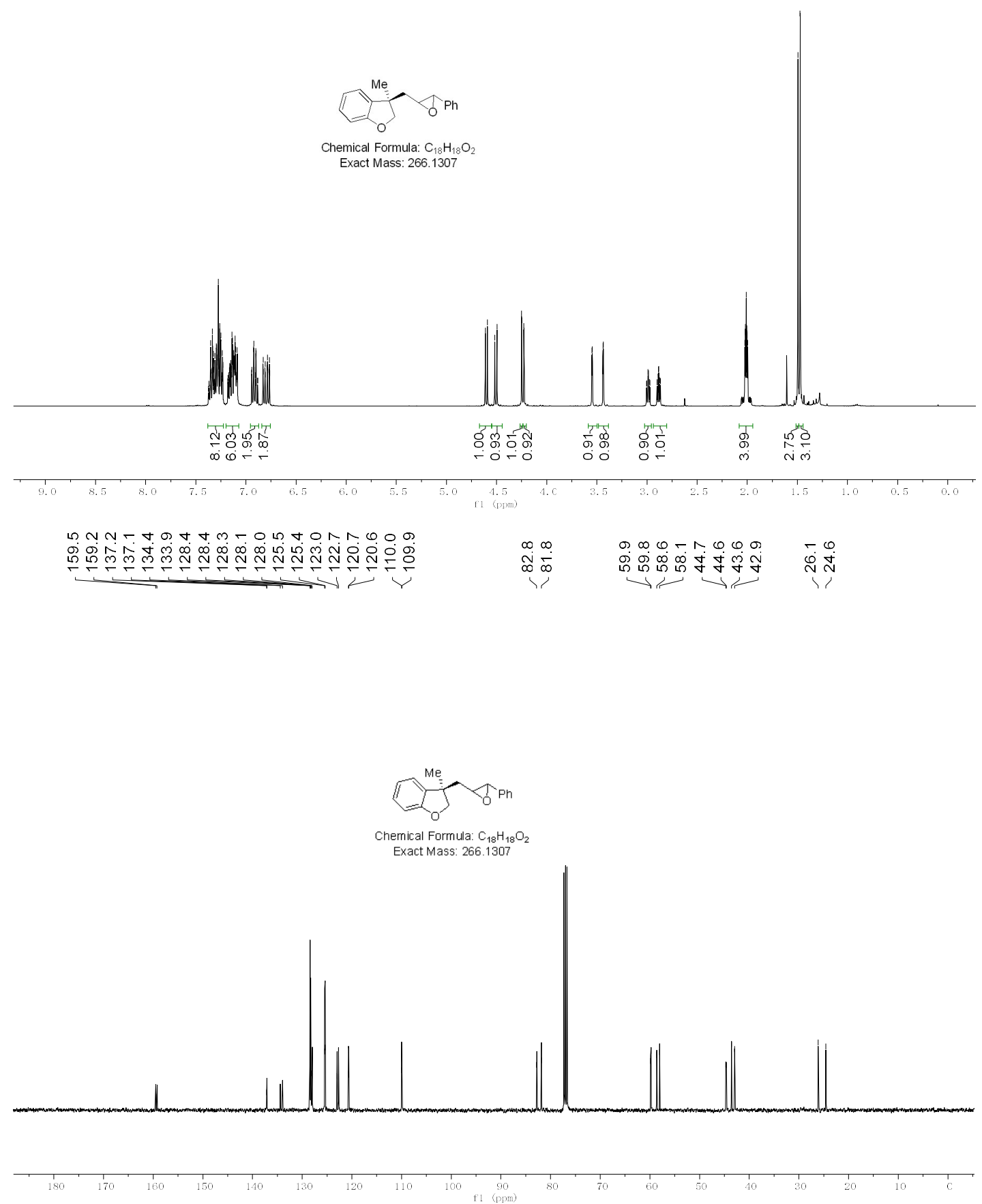

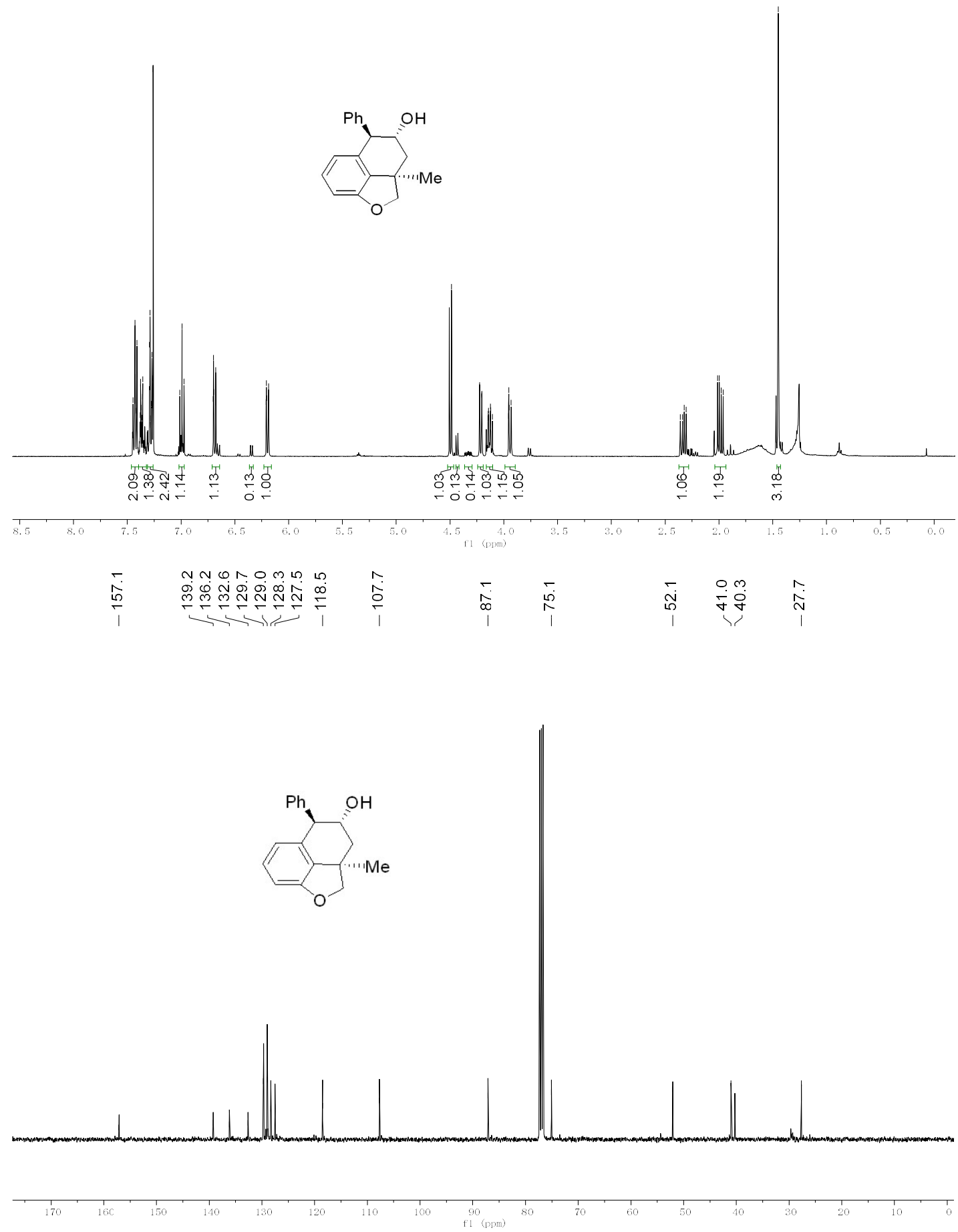

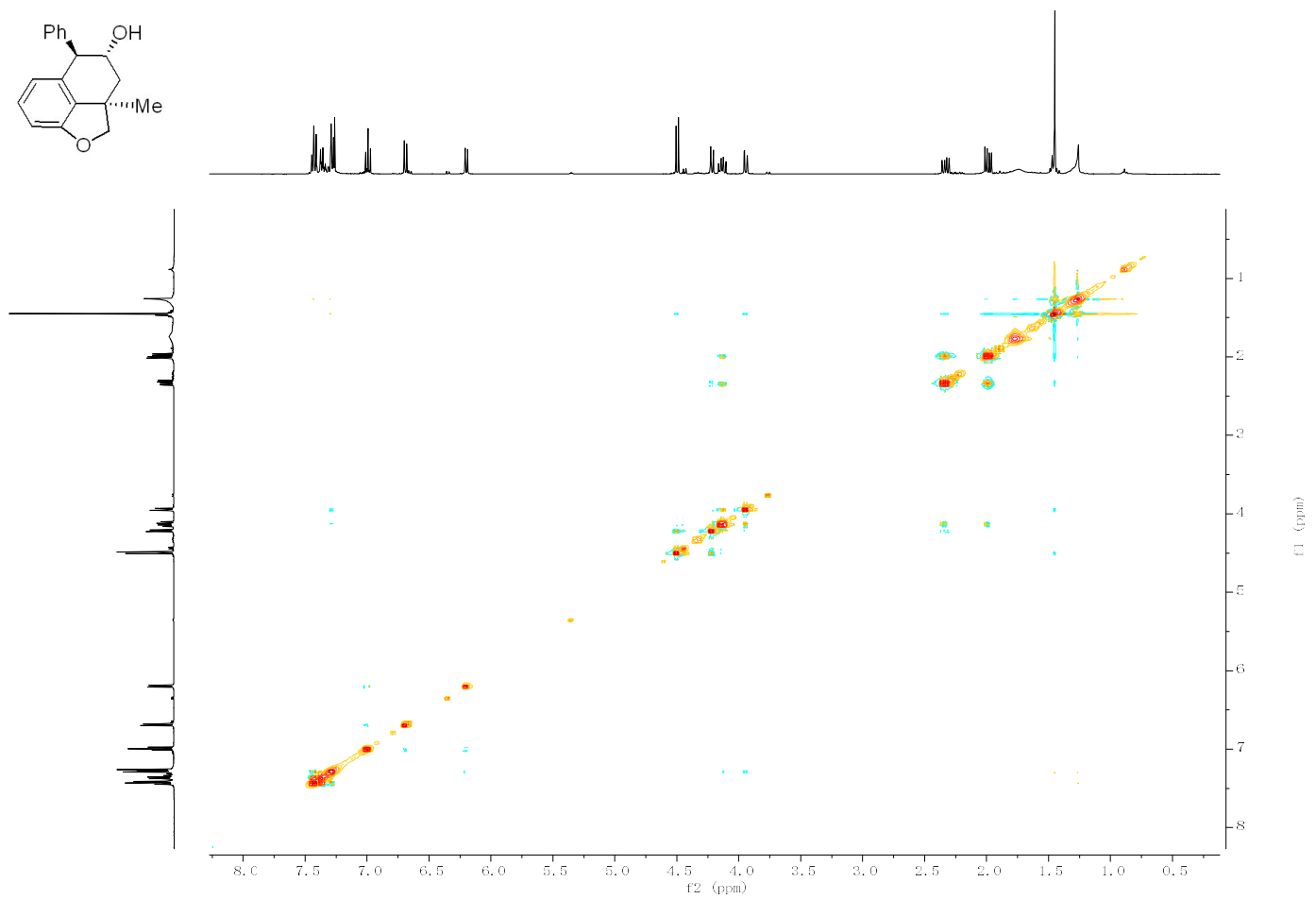


\section{References}

1. Micic, N.; Polyzos, A. Radical Carbonylation Mediated by Continuous-Flow VisibleLight Photocatalysis: Access to 2,3-Dihydrobenzofurans. Org. Lett. 2018, 20, 4663.

2. Gao, Y.; Gao, Y.; Wu, W.; Jiang, H.; Yang, X.; Liu, W.; Li, C.-J. Palladium-Catalyzed Tandem Oxidative Arylation/Olefination of Aromatic Tethered Alkenes/Alkynes. Chem. Eur. J. 2017, 23, 793.

3. Grigg, R.; Sansano, J. M.; Santhakumar, V.; Sridharan, V.; Thangavelanthum, R.; Thornton-Pett, M.; Wilson, D. Palladium catalysed tandem cyclisation-anion capture processes. Part 3. Organoboron anion transfer agents. Tetrahedron, 1997, 53, 11803. 4. Zhang, Z.; Xu, B.; Qian, Y.; Wu, L.; Wu, Y.; Zhou, L.; Liu, Y.; Zhang, L. PalladiumCatalyzed Enantioselective Reductive Heck Reactions: Convenient Access to 3,3Disubstituted 2,3-Dihydrobenzofuran. Angew. Chem. Int. Ed. 2018, 57, 10373-10377. 